Prepared in cooperation with the California State Water Resources Control Board A product of the California Groundwater Ambient Monitoring and Assessment (GAMA) Program

\title{
Groundwater-Quality Data in the Santa Cruz, San Gabriel, and Peninsular Ranges Hard Rock Aquifers Study Unit, 2011-2012: Results from the California GAMA Program
}

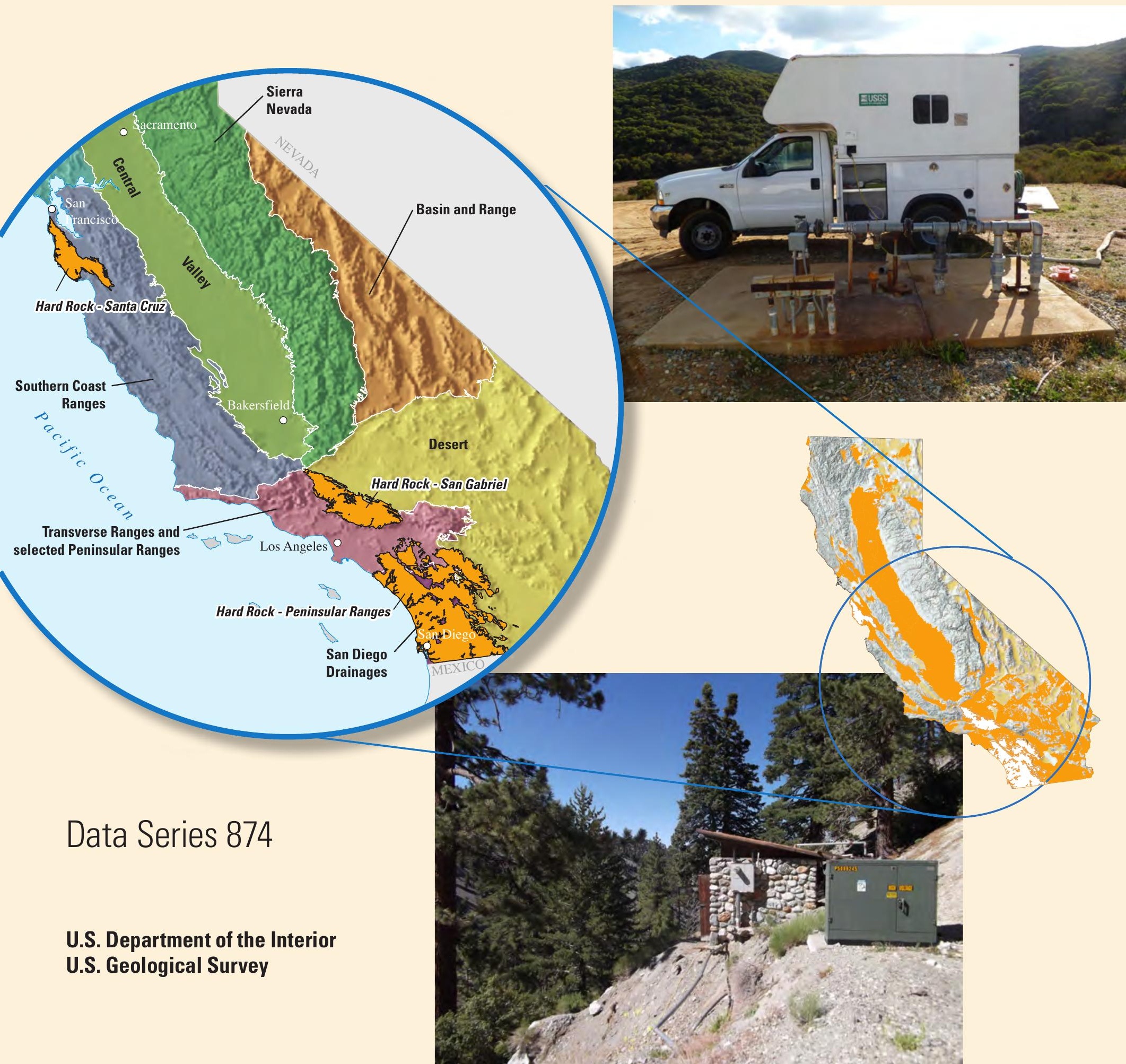


Cover photographs:

Top right: Well in San Jacinto Valley, California. (Photograph taken by Franklin Moseanko, U.S. Geological Survey).

Bottom right: Wellhouse on mountainside in Angeles National Forest. (Photograph taken by Tyler Evans, U.S. Geological Survey.) 


\section{Groundwater-Quality Data in the Santa Cruz, San Gabriel, and Peninsular Ranges Hard Rock Aquifers Study Unit, 2011-2012: Results from the California GAMA Program}

By Tracy A. Davis and Jennifer L. Shelton

A product of the California Groundwater Ambient Monitoring and Assessment (GAMA) Program

Prepared in cooperation with the California State Water Resources Control Board

Data Series 874 


\title{
U.S. Department of the Interior SALLY JEWELL, Secretary
}

\section{U.S. Geological Survey Suzette M. Kimball, Acting Director}

\author{
U.S. Geological Survey, Reston, Virginia: 2014
}

For more information on the USGS - the Federal source for science about the Earth, its natural and living resources, natural hazards, and the environment, visit http://www.usgs.gov or call 1-888-ASK-USGS.

For an overview of USGS information products, including maps, imagery, and publications, visit http://WwW.usgs.gov/pubprod

To order this and other USGS information products, visit http://store.usgs.gov

Any use of trade, firm, or product names is for descriptive purposes only and does not imply endorsement by the U.S. Government.

Although this information product, for the most part, is in the public domain, it also may contain copyrighted materials as noted in the text. Permission to reproduce copyrighted items must be secured from the copyright owner.

Suggested citation:

Davis, T.A., and Shelton, J.L., 2014, Groundwater-quality data in the Santa Cruz, San Gabriel, and Peninsular Ranges Hard Rock Aquifers study unit, 2011-2012—Results from the California GAMA Program: U.S. Geological Survey Data Series 874, 142 p., http://dx.doi.org/10.3133/ds874.

ISSN 2327-638X (Online)

ISSN 2327-0271 (Print)

ISBN 978-1-4113-3861-6 


\section{Contents}

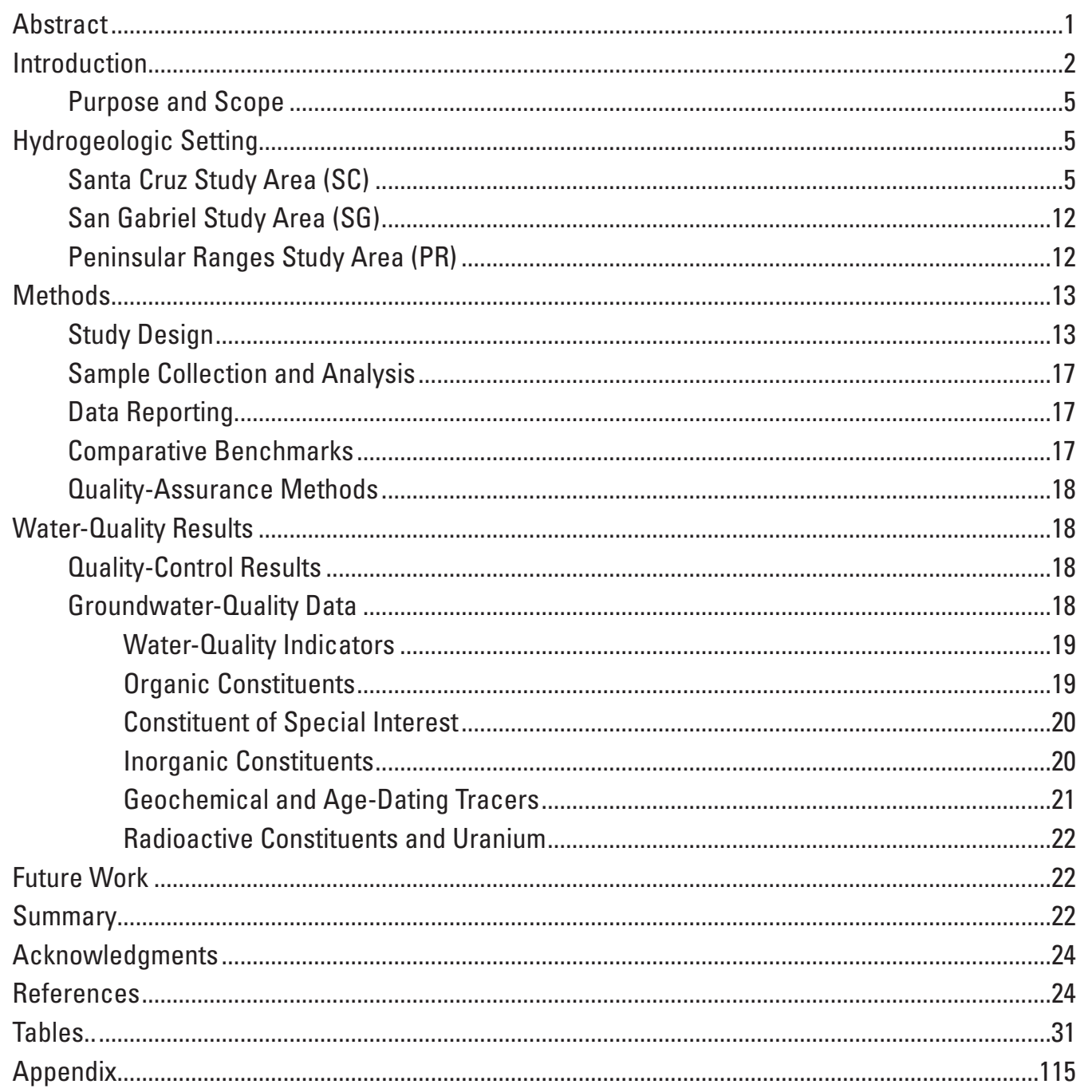




\section{Figures}

1. Map showing the hydrogeologic provinces of California and the location of the Santa Cruz, San Gabriel, and Peninsular Ranges Hard Rock Aquifers study unit, California GAMA Priority Basin Project .

2. Maps of the Santa Cruz, San Gabriel, and Peninsular Ranges study areas showing selected hydrologic and geologic features, Hard Rock study unit, California GAMA Priority Basin Project.

3. Maps of the Santa Cruz, San Gabriel, and Peninsular Ranges study areas showing the 3-kilometer buffers, locations of wells in the California Department of Public Health database, counties, major roads and cities, and hydrologic and topographic features, Hard Rock study unit, California GAMA Priority Basin Project.

4. Maps of the Santa Cruz, San Gabriel, and Peninsular Ranges study areas showing the distribution of the grid cells, locations of sampled wells, major cities, and hydrologic and topographic features, Hard Rock study unit, California GAMA Priority Basin Project.

\section{Tables}

1. Identification, sampling, and construction information for wells sampled for the Santa Cruz, San Gabriel, and Peninsular Ranges Hard Rock Aquifers study unit, March 2011 through March 2012, California GAMA Priority Basin Project.

2. Classes of chemical constituents and field water-quality indicators collected for the Santa Cruz, San Gabriel, and Peninsular Ranges Hard Rock Aquifers study unit, March 2011 through March 2012, California GAMA Priority Basin Project .....35

3A. Volatile organic compounds, primary uses or sources, reporting information, and comparative benchmarks for the U.S. Geological Survey

National Water Quality Laboratory Schedule 2020.

3B. Pesticides and pesticide degradates, primary uses or sources, reporting information, and comparative benchmarks for the U.S. Geological Survey National Water Quality Laboratory Schedule 2003.

3C. Constituent of special interest, primary uses or sources, reporting information, and comparative benchmark for analysis performed by Weck Laboratories, Inc.

3D. Trace elements, reporting information, and comparative benchmarks for the U.S. Geological Survey National Water Quality Laboratory Schedule 1948 .42

3E. Nutrients, reporting information, and comparative benchmarks for the U.S. Geological Survey National Water Quality Laboratory Schedule 2755

3F. Major and minor ions, silica, total dissolved solids, and laboratory alkalinity, reporting information, and comparative benchmarks for the U.S. Geological Survey National Water Quality Laboratory Schedule 1948.

3G. Arsenic, chromium, and iron species, reporting information, and comparative benchmarks for the U.S. Geological Survey Trace Metal Laboratory, Boulder, Colorado, analyses.. 


\section{Tables-Continued}

$3 \mathrm{H}$. Isotopic and radioactive constituents, reporting information, and comparative benchmarks for laboratory analyses..

31. Dissolved noble gases and tritium, reporting information, and comparative benchmarks for the Lawrence Livermore National Laboratory analyses.

4. Water-quality indicators in samples collected for the Santa Cruz, San Gabriel, and Peninsular Ranges Hard Rock Aquifers study unit, March 2011 through March 2012, California GAMA Priority Basin Project.

5. Volatile organic compounds detected in samples collected for the Santa Cruz, San Gabriel, and Peninsular Ranges Hard Rock Aquifers study unit, March 2011 through March 2012, California GAMA Priority Basin Project.

6. Pesticides and pesticide degradates detected in samples collected for the Santa Cruz, San Gabriel, and Peninsular Ranges Hard Rock Aquifers study unit, March 2011 through March 2012, California GAMA Priority Basin Project.

7. Perchlorate detected in samples collected for the Santa Cruz, San Gabriel, and Peninsular Ranges Hard Rock Aquifers study unit, March 2011 through March 2012, California GAMA Priority Basin Project.

8. Trace elements detected in the samples collected for the Santa Cruz, San Gabriel, and Peninsular Ranges Hard Rock Aquifers study unit, March 2011 through March 2012, California GAMA Priority Basin Project

9. Nutrients detected in samples collected for the Santa Cruz, San Gabriel, and Peninsular Ranges Hard Rock Aquifers study unit, March 2011 through March 2012, California GAMA Priority Basin Project.

10. Major and minor ions, silica, and total dissolved solids detected in samples collected for the Santa Cruz, San Gabriel, and Peninsular Ranges Hard Rock Aquifers study unit, March 2011 through March 2012, California GAMA Priority Basin Project

11. Arsenic, chromium, and iron species detected in samples collected for the Santa Cruz, San Gabriel, and Peninsular Ranges Hard Rock Aquifers study unit, March 2011 through March 2012, California GAMA Priority Basin Project.

12. Results for analyses of stable isotope ratios, carbon-14 abundance, and tritium activity in samples collected for the Santa Cruz, San Gabriel, and Peninsular Ranges Hard Rock Aquifers study unit, March 2011 through March 2012, California GAMA Priority Basin Project..

13. Results for analyses of dissolved noble gases by the Lawrence Livermore National Laboratory for samples collected for the Santa Cruz, San Gabriel, and Peninsular Ranges Hard Rock Aquifers study unit, March 2011 through March 2012, California GAMA Priority Basin Project.

14A. Radon-222 detected in samples collected for the Santa Cruz, San Gabriel, and Peninsular Ranges Hard Rock Aquifers study unit, March 2011 through March 2012, California GAMA Priority Basin Project.

14B. Gross alpha and gross beta radioactivity detected in samples collected for the Santa Cruz, San Gabriel, and Peninsular Ranges Hard Rock Aquifers study unit, March 2011 through March 2012, California GAMA Priority Basin Project.

14C. Uranium activities detected in samples collected for the Santa Cruz, San Gabriel, and Peninsular Ranges Hard Rock Aquifers study unit, March 2011 through March 2012, California GAMA Priority Basin Project. 


\section{Conversion Factors and Datums}

\section{Conversion Factors}

Inch/Pound to SI

\begin{tabular}{|c|c|c|}
\hline Multiply & By & To obtain \\
\hline inch (in.) & 2.54 & centimeter $(\mathrm{cm})$ \\
\hline foot $(\mathrm{ft})$ & 0.3048 & meter $(\mathrm{m})$ \\
\hline mile (mi) & 1.609 & kilometer $(\mathrm{km})$ \\
\hline square mile $\left(\mathrm{mi}^{2}\right)$ & 2.590 & square kilometer $\left(\mathrm{km}^{2}\right)$ \\
\hline quart (qt) & 0.95 & liter $(\mathrm{L})$ \\
\hline ounce, avoirdupois (oz) & 28.35 & $\operatorname{gram}(\mathrm{g})$ \\
\hline pound, avoirdupois (lb) & 0.4536 & kilogram (kg) \\
\hline picocurie per liter (pCi/L) & 0.037 & becquerel per liter $(\mathrm{Bq} / \mathrm{L})$ \\
\hline
\end{tabular}

SI to Inch/Pound

\begin{tabular}{lll}
\hline \multicolumn{1}{c}{ Multiply } & \multicolumn{1}{c}{ By } & \multicolumn{1}{c}{ To obtain } \\
\hline centimeter $(\mathrm{cm})$ & 0.3937 & inch (in.) \\
meter $(\mathrm{m})$ & 3.28 & foot $(\mathrm{ft})$ \\
kilometer $(\mathrm{km})$ & 0.6214 & mile $(\mathrm{mi})$ \\
square kilometer $\left(\mathrm{km}^{2}\right)$ & 0.3861 & square mile $\left(\mathrm{mi}^{2}\right)$ \\
liter $(\mathrm{L})$ & 1.057 & quart $(\mathrm{qt})$ \\
gram $(\mathrm{g})$ & 0.03527 & ounce, avoirdupois $(\mathrm{oz})$ \\
kilogram $(\mathrm{kg})$ & 2.205 & pound, avoirdupois $(\mathrm{lb})$ \\
becquerel per liter $(\mathrm{Bq} / \mathrm{L})$ & 27.03 & picocurie per liter $(\mathrm{pCi} / \mathrm{L})$ \\
\hline
\end{tabular}

Temperature in degrees Celsius $\left({ }^{\circ} \mathrm{C}\right)$ may be converted to degrees Fahrenheit $\left({ }^{\circ} \mathrm{F}\right)$ as follows:

${ }^{\circ} \mathrm{F}=\left(1.8 \mathrm{x}^{\circ} \mathrm{C}\right)+32$.

Specific conductance is given in microsiemens per centimeter at 25 degrees Celsius $(\mu \mathrm{S} / \mathrm{cm}$ at $\left.25^{\circ} \mathrm{C}\right)$.

Turbidity is given in nephelometric turbidity units (NTU).

Concentrations of chemical constituents in water are given either in milligrams per liter (mg/L) or micrograms per liter ( $\mu \mathrm{g} / \mathrm{L})$. Milligrams per liter is equivalent to parts per million (ppm), and micrograms per liter is equivalent to parts per billion $(\mathrm{ppb})$.

Concentrations of dissolved noble gases are given as the atom ratio (for helium-3/helium-4) or as cubic centimeters of gas at standard temperature and pressure per gram of water $\left(\mathrm{cm}^{3}{ }_{\text {STP }} / \mathrm{g}\right)$.

Activities of radioactive constituents in water are given in picocuries per liter (pCi/L). 


\section{Datums}

Vertical coordinate information is referenced to the North American Vertical Datum of 1988 (NAVD 88). Land-surface datum (LSD), as used in this report, refers to a horizontal plane that is approximately at land surface at each site, at a specific altitude relative to NAVD 88.

Horizontal coordinate information is referenced to the North American Datum of 1983 (NAD 83).

\section{Selected Terms and Symbols}

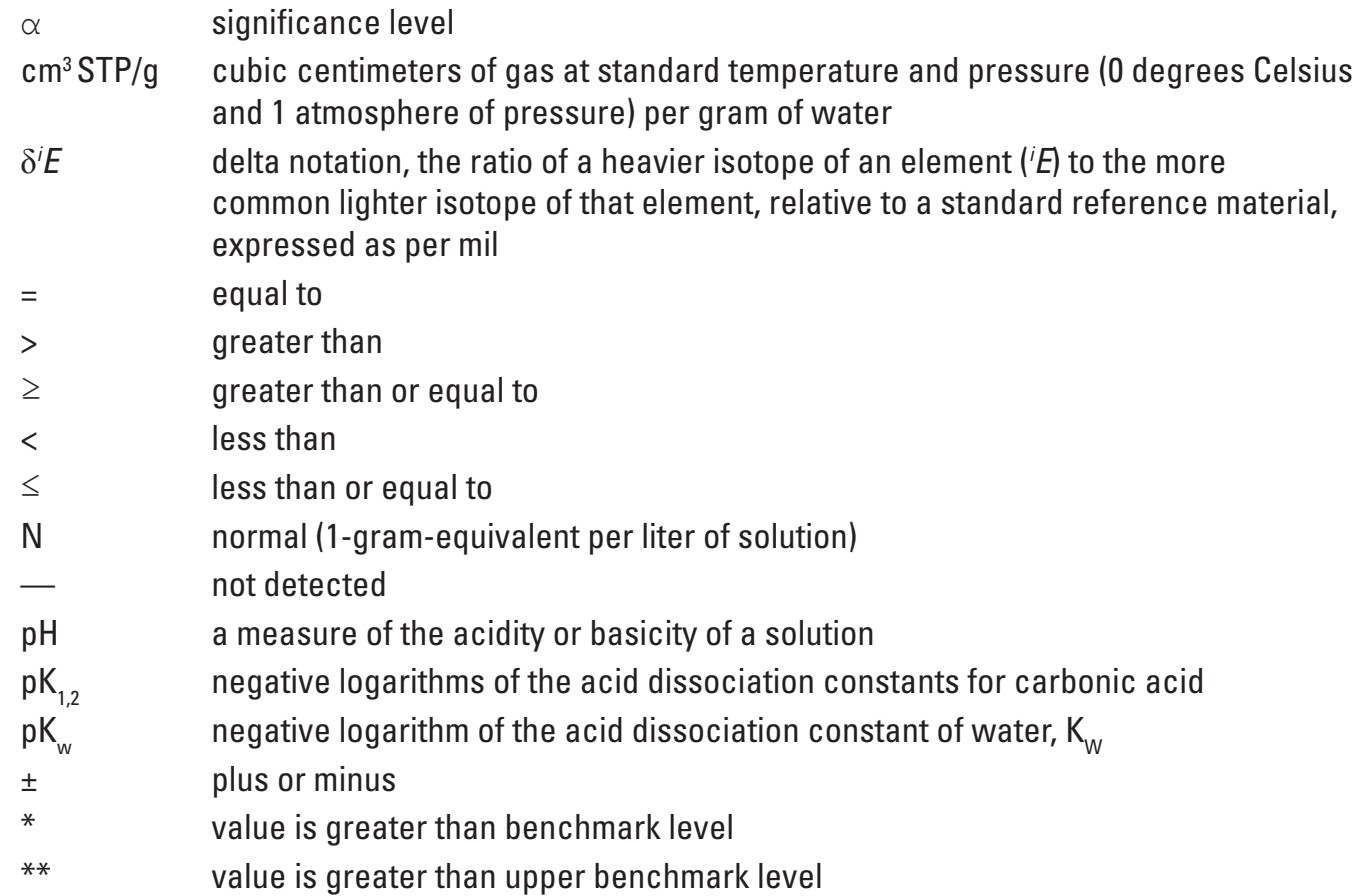

\section{Abbreviations and Acronyms}

$\begin{array}{ll}\text { AL-US } & \text { action level (USEPA) } \\ \text { APE } & \text { Alternate Place Entry program designed for USGS sampling } \\ \text { BBP } & \text { Blind Blank Project (USGS Branch of Quality Systems) } \\ \text { CASRN } & \text { Chemical Abstract Service (American Chemical Society) Registry Number }{ }^{\circledR} \\ \text { CSU } & \text { combined standard uncertainty } \\ \text { E } & \text { estimated or having a higher degree of uncertainty } \\ \text { GAMA } & \text { Groundwater Ambient Monitoring and Assessment Program } \\ \text { GPS } & \text { global positioning system } \\ \text { HAL-US } & \text { lifetime health advisory level (USEPA) } \\ \text { HPLC } & \text { high-performance liquid chromatography } \\ \text { IBSP } & \text { Inorganic Blind Sample Project (USGS Branch of Quality Systems) } \\ \text { IRL } & \text { interim reporting level } \\ \text { LRL } & \text { laboratory reporting level }\end{array}$


Abbreviations and Acronyms-Continued

\begin{tabular}{|c|c|}
\hline LSD & land-surface datum \\
\hline LT-MDL & long-term method detection level \\
\hline MCL-CA & maximum contaminant level (CDPH) \\
\hline MCL-US & maximum contaminant level (USEPA) \\
\hline MDL & method detection limit \\
\hline MRL & minimum reporting level \\
\hline MU & method uncertainty \\
\hline na & not available \\
\hline NAD & normalized absolute difference \\
\hline NFM & National Field Manual (USGS) \\
\hline NFOA & National Field Quality Assurance Program (USGS Branch of Quality Systems) \\
\hline NL-CA & notification level (CDPH) \\
\hline nv & no measured value or no value in category \\
\hline NWIS & National Water Information System (USGS) \\
\hline PBP & Priority Basin Project (USGS GAMA) \\
\hline PCFF & Personal Computer Field Form program designed for USGS sampling \\
\hline $\mathrm{QA}$ & quality assurance \\
\hline $\mathrm{QC}$ & quality control \\
\hline RPD & relative percent difference \\
\hline RSD & relative standard deviation \\
\hline RSD5-US & USEPA risk-specific dose at a risk factor of $10^{-5}$ \\
\hline SAFZ & San Andreas Fault Zone \\
\hline SD & standard deviation \\
\hline SMCL-CA & secondary maximum contaminant level (CDPH) \\
\hline SMCL-US & secondary maximum contaminant level (USEPA) \\
\hline SRL & $\begin{array}{l}\text { study reporting level (concentration cutoff for applying the } \leq \text { symbol in reporting } \\
\text { results) }\end{array}$ \\
\hline $\mathrm{ssL}_{\mathrm{c}}$ & sample-specific critical level \\
\hline UV-Vis & ultraviolet-visible \\
\hline VPDB & $\begin{array}{l}\text { Vienna Pee Dee Belemnite (the international reference standard for carbon } \\
\text { isotopes) }\end{array}$ \\
\hline VSMOW & $\begin{array}{l}\text { Vienna Standard Mean Ocean Water (an isotopic water standard defined in } 1968 \\
\text { by the International Atomic Energy Agency) }\end{array}$ \\
\hline
\end{tabular}

\section{Organizations}

BOS Branch of Quality Systems (USGS)

CDPH California Department of Public Health

CDPR California Department of Pesticide Regulation

CDWR California Department of Water Resources

LLNL Lawrence Livermore National Laboratory

NAWQA National Water-Quality Assessment Program (USGS)

NELAP National Environmental Laboratory Accreditation Program 


\section{Organizations-Continued}

NWQL National Water Quality Laboratory (USGS)

SITL Stable Isotope and Tritium Laboratory, Menlo Park, California (USGS)

SWRCB California State Water Resources Control Board

USEPA U.S. Environmental Protection Agency

USGS U.S. Geological Survey

\section{Selected Constituent Names}

$\begin{array}{ll}\mathrm{B} & \text { boron } \\ \mathrm{C} & \text { carbon } \\ \mathrm{CaCO}_{3} & \text { calcium carbonate } \\ \mathrm{CFC} & \text { chlorofluorocarbon } \\ \mathrm{CO}_{3}{ }^{2-} & \text { carbonate ion } \\ \mathrm{H}^{-} & \text {hydrogen } \\ \mathrm{HCO}_{3}- & \text { bicarbonate ion } \\ \mathrm{MTBE} & \text { methyl tert-butyl ether } \\ 0 & \text { oxygen } \\ \mathrm{PCE} & \text { perchloroethene (tetrachloroethene) } \\ \mathrm{PVC} & \text { polyvinyl chloride } \\ \mathrm{Sr} & \text { strontium } \\ \text { TDS } & \text { total dissolved solids } \\ \text { THM } & \text { trihalomethane } \\ \text { VOC } & \text { volatile organic compound }\end{array}$





\title{
Groundwater-Quality Data in the Santa Cruz, San Gabriel, and Peninsular Ranges Hard Rock Aquifers Study Unit, 2011-2012: Results from the California GAMA Program
}

\author{
By Tracy A. Davis and Jennifer L. Shelton
}

\section{Abstract}

Groundwater quality in the 2,400-square-mile Santa Cruz, San Gabriel, and Peninsular Ranges Hard Rock Aquifers (Hard Rock) study unit was investigated by the U.S. Geological Survey (USGS) from March 2011 through March 2012, as part of the California State Water Resources Control Board (SWRCB) Groundwater Ambient Monitoring and Assessment (GAMA) Program's Priority Basin Project (PBP). The GAMA-PBP was developed in response to the California Groundwater Quality Monitoring Act of 2001 and is being conducted in collaboration with the SWRCB and Lawrence Livermore National Laboratory (LLNL). The Hard Rock study unit was the 35th study unit to be sampled as part of the GAMA-PBP.

The GAMA Hard Rock study was designed to provide a spatially unbiased assessment of untreatedgroundwater quality in the primary aquifer system and to facilitate statistically consistent comparisons of untreatedgroundwater quality throughout California. The primary aquifer system is defined as those parts of the aquifers corresponding to the perforation intervals of wells listed in the California Department of Public Health (CDPH) waterquality-monitoring database for the Hard Rock study unit. Groundwater quality in the primary aquifer system may differ from the quality in the shallower or deeper water-bearing zones; shallow groundwater may be more vulnerable to surficial contamination.

In the Hard Rock study unit, groundwater samples were collected from 112 wells and springs in 3 study areas (the Santa Cruz, the San Gabriel, and the Peninsular Ranges) in San Mateo, Santa Clara, Santa Cruz, San Benito, Los Angeles, Orange, Riverside, San Bernardino, and San Diego Counties. Eighty-three wells and 11 springs were selected by using a spatially distributed, randomized grid-based method to provide statistical representation of the study unit (grid wells), and 15 wells and 3 springs were selected to aid in evaluation of water-quality issues (understanding wells).
The groundwater samples were analyzed for field waterquality indicators; organic constituents; one constituent of special interest (perchlorate); naturally occurring inorganic constituents; and radioactive constituents. Naturally occurring isotopes and dissolved noble gases were also measured to help identify the sources and ages of the sampled groundwater. In total, 209 constituents and water-quality indicators were measured.

Three types of quality-control samples (blanks, replicates, and matrix spikes) were collected at approximately 10 percent of the wells in the Hard Rock study unit, and the results for these samples were used to evaluate the quality of the data for the groundwater samples. Blanks rarely contained detectable concentrations of any constituent, suggesting that contamination from sample collection procedures was not a significant source of bias in the data for the groundwater samples. Replicate samples generally were within the limits of acceptable analytical reproducibility. Median matrixspike recoveries were within the acceptable range (70 to 130 percent) for approximately 92 percent of the compounds.

This study did not attempt to evaluate the quality of water delivered to consumers; after withdrawal from the ground, untreated groundwater typically is treated, disinfected, and (or) blended with other waters to maintain water quality. Regulatory benchmarks apply to water that is served to the consumer, not to untreated groundwater. However, to provide some context for the results, concentrations of constituents measured in the untreated groundwater were compared with regulatory and nonregulatory health-based benchmarks established by the U.S. Environmental Protection Agency (USEPA) and CDPH, and to nonregulatory benchmarks established for aesthetic concerns by the CDPH. Comparisons between data collected for this study and benchmarks for drinking water are for illustrative purposes only and are not indicative of compliance or non-compliance with those benchmarks. 
All organic constituents and most inorganic constituents that were detected in groundwater samples from the 112 wells in the Hard Rock study unit were detected at concentrations less than drinking-water benchmarks.

Of the 149 organic and special-interest constituents, 34 were detected in groundwater samples; concentrations of all detected constituents were less than regulatory and nonregulatory health-based benchmarks. In total, VOCs were detected in 44 percent of the 94 grid wells sampled, pesticides and pesticide degradates were detected in 18 percent, and perchlorate was detected in 48 percent.

Trace elements, nutrients, major and minor ions, and radioactive constituents were sampled for at 94 grid wells; most detected concentrations were less than health-based benchmarks. Exceptions in the Hard Rock study unit grid wells include 3 detections of arsenic greater than the USEPA maximum contaminant level (MCL-US) of 10 micrograms per liter $(\mu \mathrm{g} / \mathrm{L}), 3$ detections of boron greater than the CDPH notification level (NL-CA) of 1,000 $\mu \mathrm{g} / \mathrm{L}, 2$ detections of molybdenum greater than the USEPA lifetime health advisory level (HAL-US) of $40 \mu \mathrm{g} / \mathrm{L}, 2$ detections of nitrite plus nitrate (as nitrogen) greater than the MCL-US of 10 milligrams per liter $(\mathrm{mg} / \mathrm{L}), 3$ detections of fluoride greater than the CDPH maximum contaminant level (MCL-CA) of $2 \mathrm{mg} / \mathrm{L}$, 5 detections of radon-222 greater than the proposed MCL-US of 4,000 picocuries per liter ( $\mathrm{pCi} / \mathrm{L})$, and 11 detections of unadjusted gross alpha radioactivity greater than the MCL-US of $15 \mathrm{pCi} / \mathrm{L}$. Seven of the 11 samples having unadjusted gross alpha activity greater than the MCL-US also had total uranium concentrations greater than the MCL-US of $30 \mu \mathrm{g} / \mathrm{L}$ and (or) uranium activities greater than the MCL-CA of $20 \mathrm{pCi} / \mathrm{L}$.

Results for constituents with nonregulatory benchmarks set for aesthetic concerns showed that iron concentrations greater than the $\mathrm{CDPH}$ secondary maximum contaminant level (SMCL-CA) of $300 \mu \mathrm{g} / \mathrm{L}$ were detected in samples from 19 grid wells. Manganese concentrations greater than the SMCL-CA of $50 \mu \mathrm{g} / \mathrm{L}$ were detected in 27 grid wells. Chloride was detected at a concentration greater than the SMCL-CA upper benchmark of $500 \mathrm{mg} / \mathrm{L}$ in one grid well. TDS concentrations in three grid wells were greater than the SMCL-CA upper benchmark of $1,000 \mathrm{mg} / \mathrm{L}$.

\section{Introduction}

About one-half of the water used for public and domestic drinking-water supply in California is groundwater (Kenny and others, 2009). To assess the quality of ambient groundwater in aquifers used for public drinking-water supply and to establish a baseline groundwater-quality monitoring program, the California State Water Resources Control Board (SWRCB), in cooperation with the U.S. Geological Survey (USGS) and Lawrence Livermore National Laboratory (LLNL), implemented the Groundwater Ambient Monitoring and Assessment (GAMA) Program (California State Water Resources Control Board, 2011). The GAMA Program was initiated by the SWRCB in 2000 and later expanded by the Groundwater Quality Monitoring Act of 2001 (State of California, 2001a, 2001b, Sections 10780-10782.3 of the California Water Code, Assembly Bill 599). The main goals of the GAMA Program are to improve groundwater monitoring and to increase the availability of groundwater-quality data to the public.

The GAMA Program currently consists of four projects: (1) the GAMA Priority Basin Project (PBP) conducted by the USGS (U.S. Geological Survey, 2013a); (2) the GAMA Domestic Well Project conducted by the SWRCB; (3) the GAMA Special Studies Project conducted by LLNL; and (4) GeoTracker GAMA conducted by the SWRCB. The GAMA-PBP primarily focuses on the deep part of the groundwater resource, which is typically used for public drinking-water supply. The GAMA Domestic Well Project generally focuses on the shallow aquifer systems, which may be particularly at risk as a result of surficial contamination. The GAMA Special Studies Project focuses on using research methods to help explain the source, fate, transport, and occurrence of chemicals that can affect groundwater quality. GeoTracker GAMA is a web-based system that displays groundwater information using Google Maps and includes analytical tools and reporting features to assess water quality. The Geotracker GAMA database stores groundwater-quality data and related reports collected by other State agencies, such as the California Department of Public Health (CDPH), the California Department of Water Resources (CDWR), and the California Department of Pesticide Regulation (CDPR), and data collected by the SWRCB and Regional Boards from environmental monitoring wells at contaminated or remediated sites (California State Water Resources Control Board, 2009).

The GAMA-PBP assesses groundwater quality in key groundwater basins and other areas that account for more than 95 percent of groundwater used for public supply in the State. The USGS, in collaboration with the SWRCB, developed the monitoring plan for the project to assess groundwater basins through direct and other statistically reliable sample approaches (Belitz and others, 2003; California State Water Resources Control Board, 2003). Additional partners in the GAMA-PBP include LLNL, CDPH, CDWR, CDPR, local water agencies, and well owners (Kulongoski and Belitz, 2004). Participation in the project is entirely voluntary.

In order to delineate areas for evaluation as part of the GAMA-PBP, the range of hydrologic, geologic, and climatic 
conditions in California needed to be considered. Belitz and others (2003) partitioned the State into 10 hydrogeologic provinces, each with distinctive hydrologic, geologic, and climatic characteristics: Modoc Plateau and Cascades, Klamath Mountains, Northern Coast Ranges, Central Valley, Sierra Nevada, Basin and Range, Southern Coast Ranges, Transverse and Selected Peninsular Ranges, Desert, and San Diego Drainages (fig. 1). These 10 hydrogeologic provinces include groundwater basins and subbasins designated by the CDWR (California Department of Water Resources, 2003). Groundwater basins and subbasins generally consist of relatively permeable, unconsolidated deposits of alluvial origin. Eighty percent of California's approximately 16,000 active and standby drinking-water wells listed in the statewide water-quality monitoring database maintained by the CDPH (hereinafter referred to as CDPH wells) are located in groundwater basins and subbasins within the 10 hydrogeologic provinces. Groundwater basins and subbasins were prioritized for sampling on the basis of the number of CDPH wells in the basin, with secondary consideration given to municipal groundwater use, agricultural pumping, the number of formerly leaking underground fuel tanks, and the number of square-mile sections with registered pesticide applications (Belitz and others, 2003). Of the 472 basins and subbasins designated by the CDWR, 116 were identified as priority basins. These priority basins contain approximately 95 percent of the CDPH wells located in groundwater basins. In addition, some areas outside of the defined groundwater basins were included to achieve representation of the 20 percent of the CDPH wells not located in the groundwater basins. The priority basins, selected other basins, and selected areas outside of basins were grouped into 35 study units for sampling. The Santa Cruz, San Gabriel, and Peninsular Ranges Hard Rock Aquifers study unit (hereinafter referred to as the Hard Rock study unit) contains a large number of CDPH wells outside of the CDWR-defined groundwater basins. The Hard Rock study unit includes hard rock areas located in the Southern Coast Ranges, Transverse and Selected Peninsular Ranges, San Diego Drainages, and Desert hydrogeologic provinces (fig. 1).

The data collected in each study unit are used for three types of water-quality assessments: (1) Status: assessment of the current quality of the groundwater resource;

(2) Understanding: identification of the natural and human factors affecting groundwater quality; and (3) Trends: detection of changes in groundwater quality over time (Kulongoski and Belitz, 2004). The assessments are intended to characterize the quality of groundwater in the primary aquifer system of the study units, not the treated drinking water delivered to consumers by water purveyors. The primary aquifer system is defined as that part of the aquifers corresponding to the perforated depth intervals of wells listed in the CDPH database for the study units. The CDPH database lists wells used for public drinking-water supplies and includes wells from systems classified as community (such as those in cities, towns, and mobile-home parks), non-transient and non-community (such as those in schools, workplaces, and restaurants), and transient and non-community (such as those in campgrounds and parks). Collectively, the CDPH refers to these wells as "public-supply" wells. Groundwater quality in the primary aquifer system may differ from the quality in the shallow or deep parts of the aquifer systems. In particular, shallow groundwater may be more vulnerable to surface contamination. As a result, samples from shallow wells (such as many private domestic wells and environmental monitoring wells) can have higher concentrations of constituents (such as volatile organic compounds [VOCs] and nitrate) from anthropogenic sources than samples from wells screened in the underlying primary aquifer system (for example, Landon and others, 2010).

The GAMA-PBP is unique in California because it includes many chemical analyses that are not otherwise available in statewide water-quality monitoring datasets. Groundwater samples collected for the GAMA-PBP are typically analyzed for approximately 300 chemical constituents by using methods with lower detection limits than required by the $\mathrm{CDPH}$ for regulatory monitoring of drinking-water wells. These analyses will be especially useful for providing an early indication of changes in groundwater quality. In addition, the GAMA-PBP analyzes samples for a suite of constituents more extensive than that required by $\mathrm{CDPH}$ and for a suite of chemical and isotope tracers for exploring hydrologic and geochemical processes. A broad understanding of groundwater composition is useful for identifying the natural and human factors affecting water quality. Understanding the occurrence and distribution of chemical constituents of significance to water quality is important for the long-term management and protection of groundwater resources.

Published and quality-assured data collected for the GAMA Program are stored in the web-based Geotracker GAMA database (California State Water Resources Control Board, 2009) and in the web-based USGS National Water Information System (U.S. Geological Survey, 2014).

This USGS Data Series Report is similar to other USGS Data Series Reports written for the GAMA-PBP study units sampled to date. Data Series Reports and additional reports (Scientific Investigations Reports and Fact Sheets) addressing the status, understanding, and trends aspects of the waterquality assessments of each study unit are available from the U.S. Geological Survey (2013b) at http://ca.water.usgs.gov/ projects/gama/includes/GAMA publications.html. 


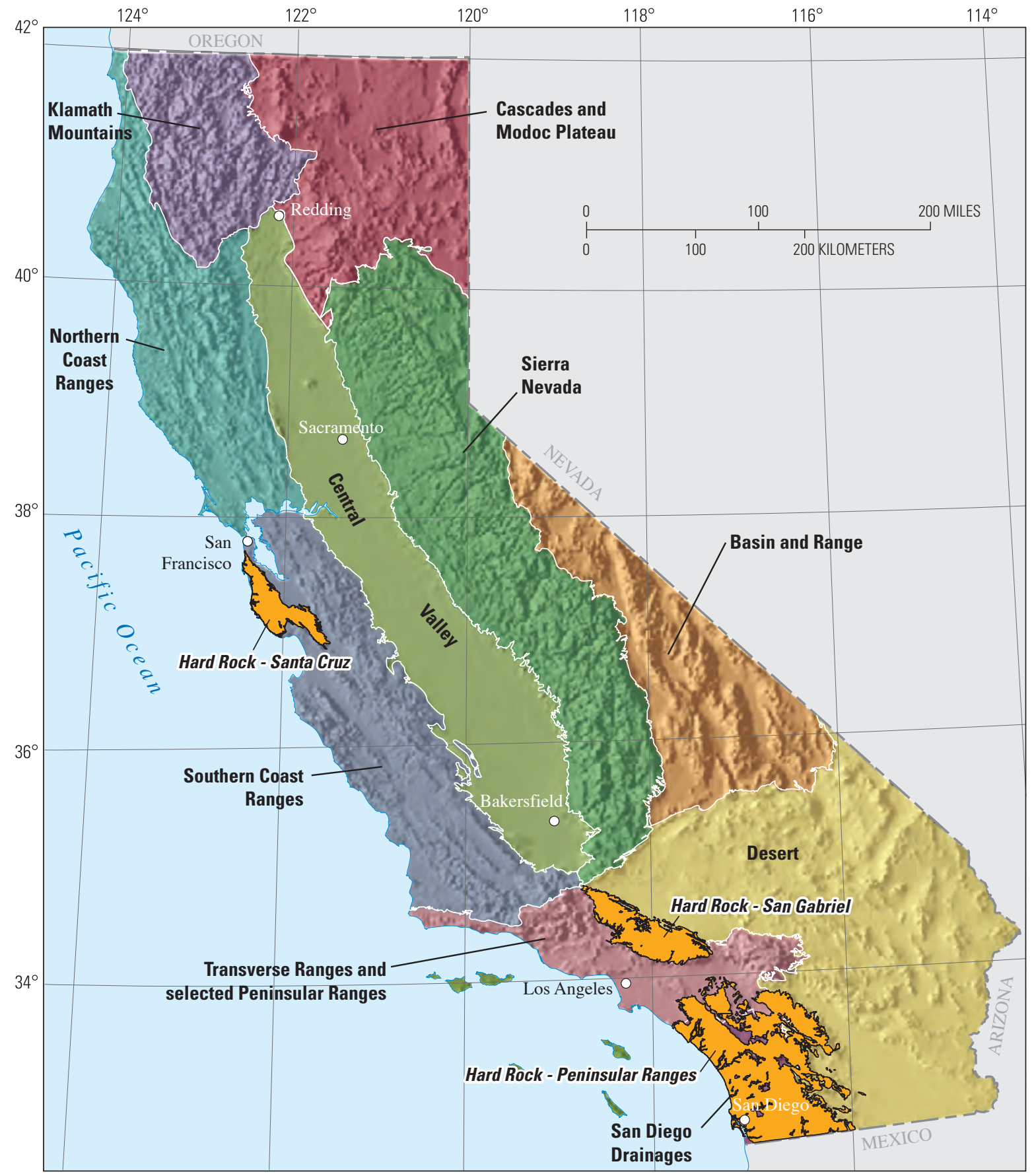

Shaded relief derived from U.S. Geological Survey

Provinces from Belitz and others, 2003 National Elevation Dataset, 2006,

Albers Equal Area Conic Projection

Figure 1. Hydrogeologic provinces of California and the location of the Santa Cruz, San Gabriel, and Peninsular Ranges Hard Rock Aquifers (Hard Rock) study unit, California GAMA Priority Basin Project. 


\section{Purpose and Scope}

The purposes of this report are to describe (1) the study design, including the hydrogeologic setting of the Hard Rock study unit and the study methods; (2) the analytical results for groundwater samples collected in the Hard Rock study unit; and (3) the results of QC analyses. Groundwater samples were collected and analyzed for field water-quality indicators; organic, special-interest, inorganic, and radioactive constituents; naturally occurring isotopes; and dissolved noble gases. The water-quality data presented in this report were compared to State and Federal drinking-water regulatory and other nonregulatory standards that are applied to treated drinking water. Regulatory and nonregulatory benchmarks considered for this report are those established by the U.S. Environmental Protection Agency (USEPA) and the $\mathrm{CDPH}$. Discussion of the factors that influence the distribution and occurrence of the constituents detected in groundwater samples will be the subject of subsequent publications.

\section{Hydrogeologic Setting}

The Hard Rock study unit consists of selected portions of California's mountainous regions outside of the CDWRdefined groundwater basins. Separated by up to 200 miles of land, the three study areas of the Hard Rock study unit — Santa Cruz (SC), San Gabriel (SG), and Peninsular Ranges (PR) each form distinct hydrogeologic settings (fig. 2). The San Andreas Fault Zone (SAFZ) forms the eastern boundary and structurally dominates the geology for all three of the study areas in the study unit. Groundwater is used extensively for public and domestic drinking-water supplies in the Hard Rock study unit, and much of this groundwater comes from aquifers in granitic, metamorphic, or lithified sedimentary rocks, rather than from sediment deposits in groundwater basins. These rocks typically have low permeability except where they are extensively fractured. The three-dimensional complexity and variability of fracture systems can cause well yields and water quality to vary widely on a local scale. Recharge to hard-rock aquifers occurs by direct infiltration of precipitation and snow melt and by infiltration from lakes and streams (California Department of Water Resources, 2011).

\section{Santa Cruz Study Area (SC)}

The Santa Cruz study area of the Hard Rock study unit is located in the Southern Coast Ranges hydrogeologic province (fig. 1) and includes parts of San Mateo, Santa Clara, Santa Cruz, and San Benito Counties (fig. 3A). The study area contains the Santa Cruz Mountains, which are approximately 75 miles in length and up to 15 miles wide. The study area boundaries were defined primarily by the extent of the igneous, metamorphic, and Tertiary-age sedimentary rocks that compose the mountains (fig. 2A). Areas mapped as Quaternary alluvium and surficial deposits or Plio-Pleistocene sediments were excluded. Several small areas of rock along the eastern boundary were excluded because they were included in the GAMA-PBP San Francisco Bay study unit (Ray and others, 2009), and a large area of rock along the southern boundary was excluded because it was included in the GAMA-PBP Monterey Bay and Salinas Valley study unit (Kulongoski and Belitz, 2007).

The SC study area is divided into two geologically distinct regions by the SAFZ and Sargent fault (fig. $2 A$ ). Rocks to the west of these faults are mostly lithified marine sediments of Tertiary age with lesser amounts of granitic rocks of Mesozoic age and older metamorphic rocks, whereas rocks to the east of these faults are primarily Mesozoic metavolcanic and metasedimentary rocks of the Franciscan Complex. Sub-parallel to the SAFZ, the Butano and Zayante-Vergeles faults cut into the marine sediment. The western edge of the study area is intersected by the San Gregorio fault, which originates off the coast of San Francisco and extends south where it terminates in Big Sur (U.S. Geological Survey, 2010). The sources of surface-water discharge for the study area are several creeks flowing from the mountains out to the Pacific Ocean, Monterey Bay, or San Francisco Bay; most notably are the Pescadero Creek, San Lorenzo River, and Pajaro River.

The SC study area is characterized as a temperate rainforest, with a cool, wet climate conducive to redwood forests. The National Climatic Data Center (NCDC) station located in Ben Lomond (fig. 2A) measured an average annual temperature of 55 degrees Fahrenheit $\left({ }^{\circ} \mathrm{F}\right)(13$ degrees Celsius $\left[{ }^{\circ} \mathrm{C}\right]$ ) for 2011. Average annual precipitation was 38 inches (U.S. Department of Commerce, National Climatic Data Center, 2011). Precipitation primarily occurs as rain during the winter and early spring and as fog cover in the summer months. Snow may occur in the winter months at the higher elevations. The highest peak in the Santa Cruz Mountains is the Loma Prieta Peak at an elevation of about 3,800 feet. 


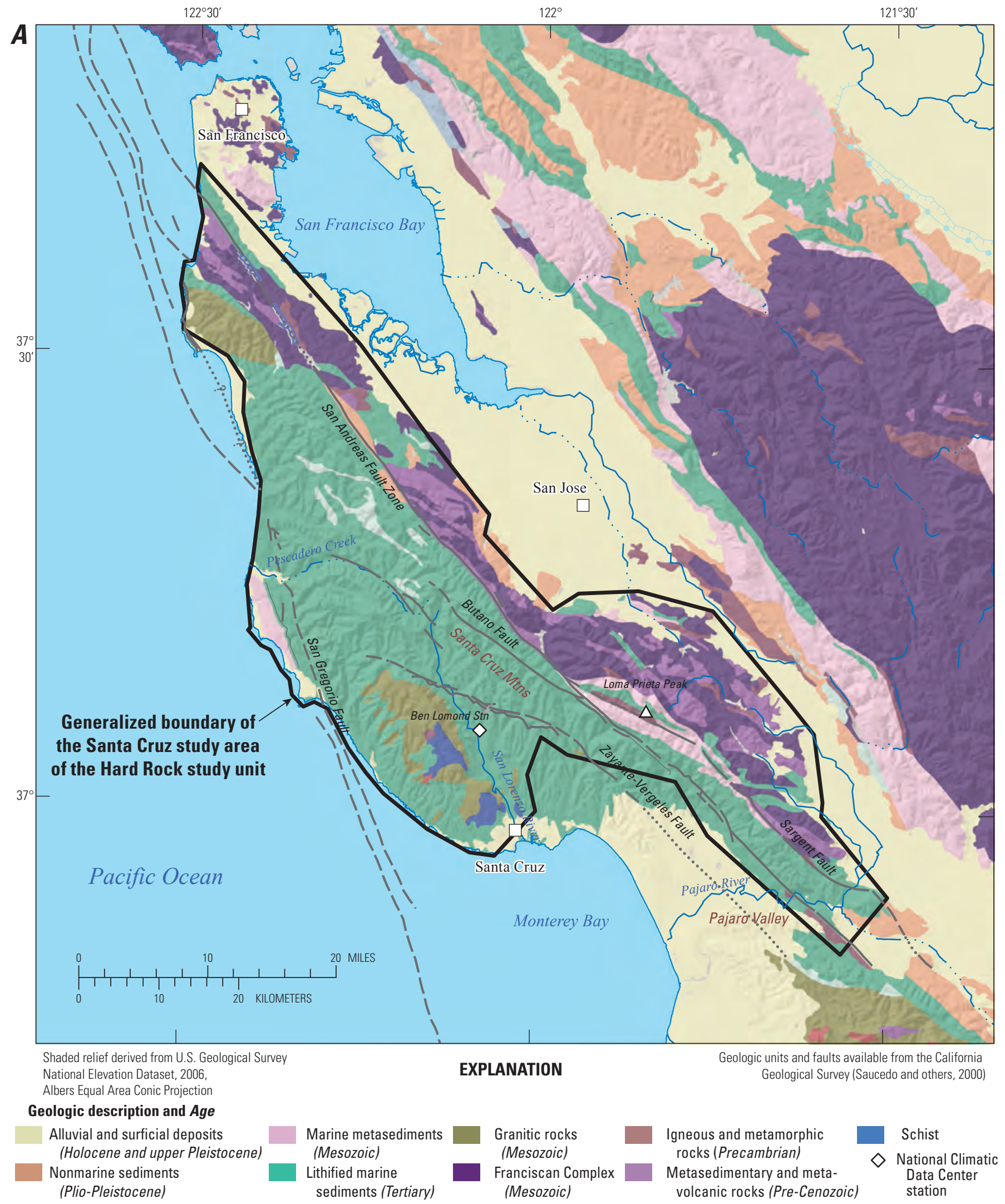

Figure 2. (A) Santa Cruz, $(B)$ San Gabriel, and $(C)$ Peninsular Ranges study areas showing selected hydrologic and geologic features, Hard Rock study unit, California GAMA Priority Basin Project. 


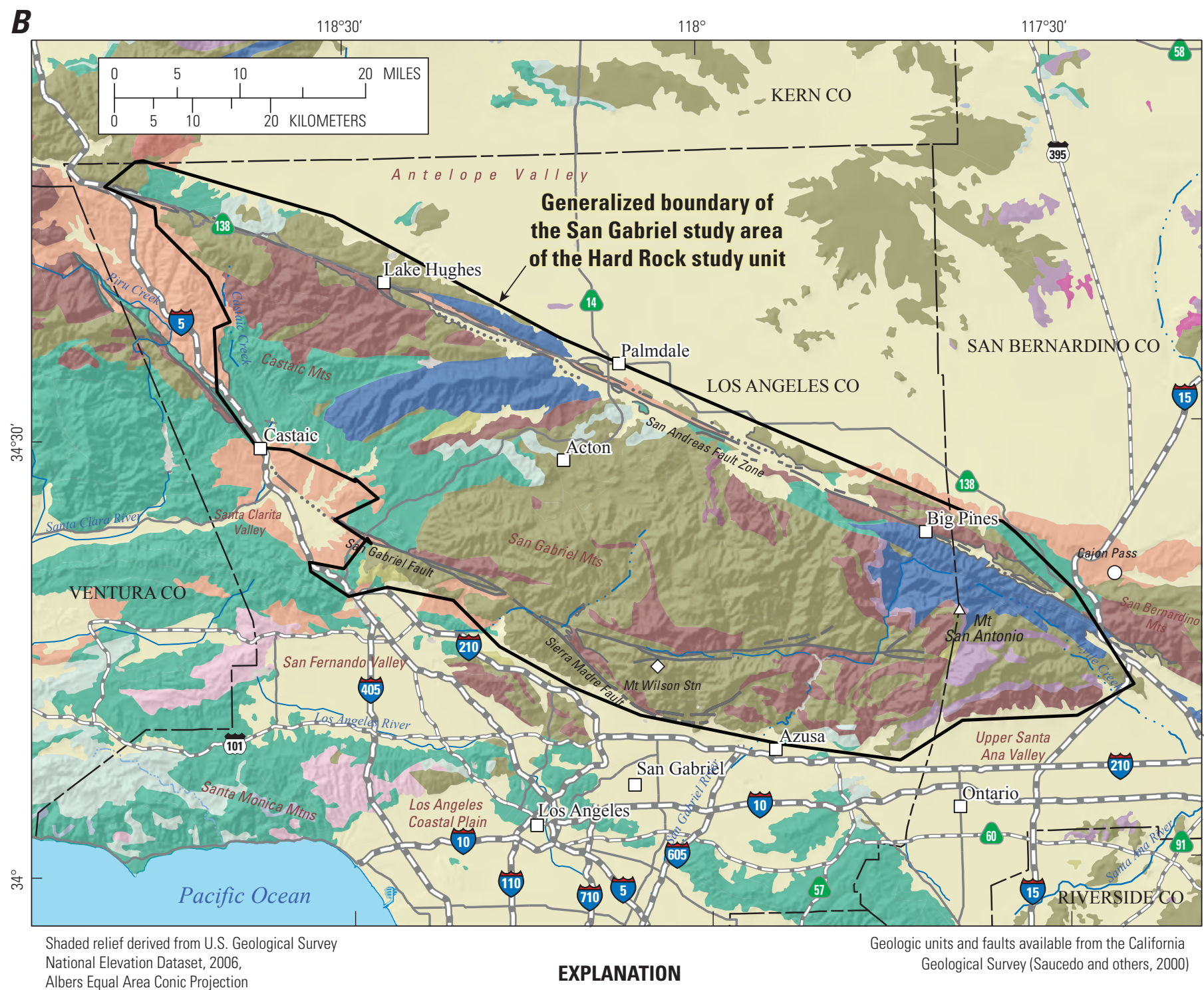

Geologic description and Age

Alluvial and surficial deposits (Holocene and upper Pleistocene)

Nonmarine sediments (Plio-Pleistocene)

$\diamond$ National Climatic Data Center station
Marine metasediments (Mesozoic)

Lithified marine sediments (Tertiary)

Pyroclastic and volcanic mudflow (Cenozoic)
Granitic rocks (Mesozoic)

Schist

Marine metasedimentary rocks (Paleozoic)
Igneous and metamorphic rocks (Precambrian)

Metasedimentary and metavolcanic rocks (Pre-Cenozoic)

Figure 2. - Continued 


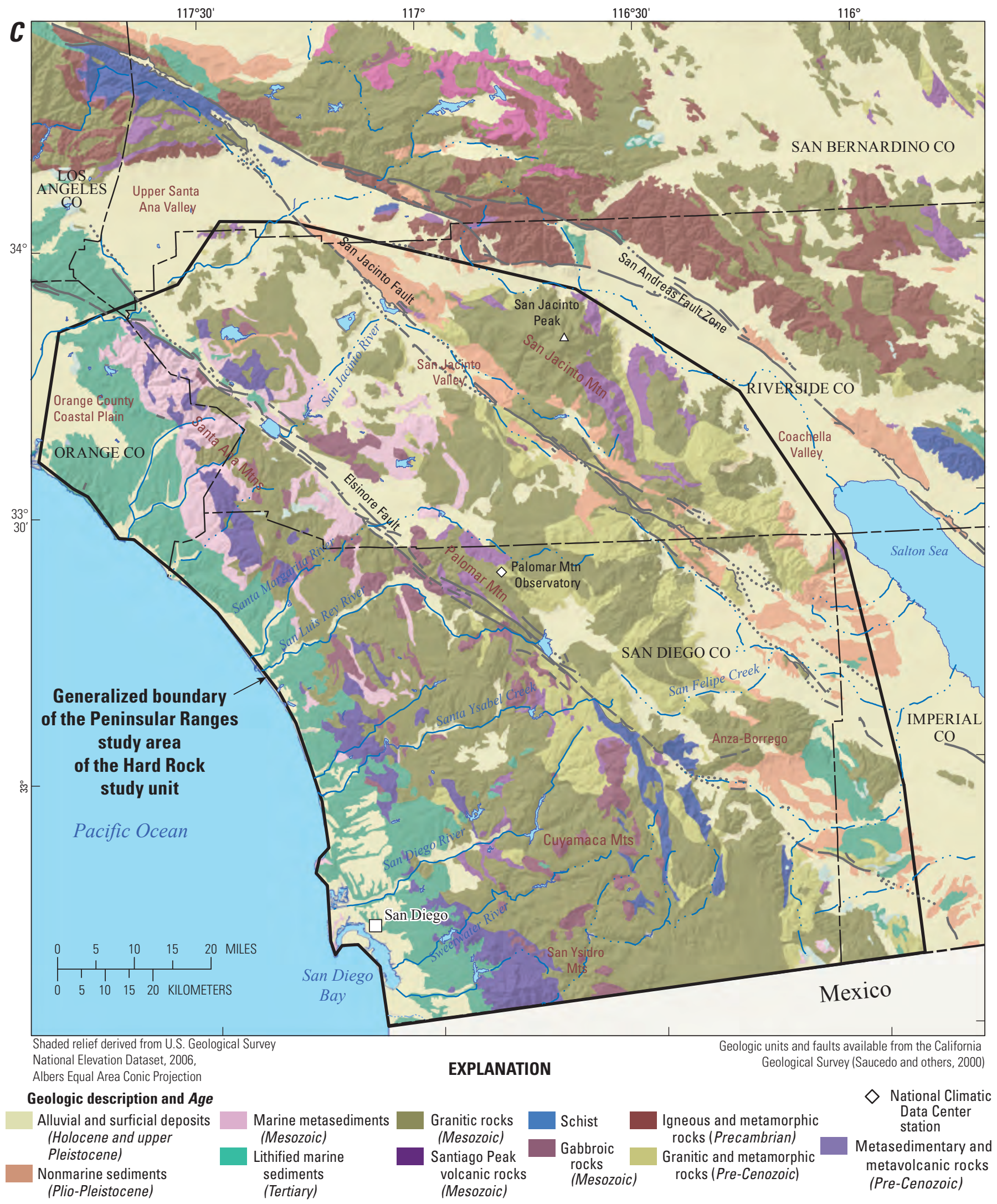

Figure 2. - Continued 


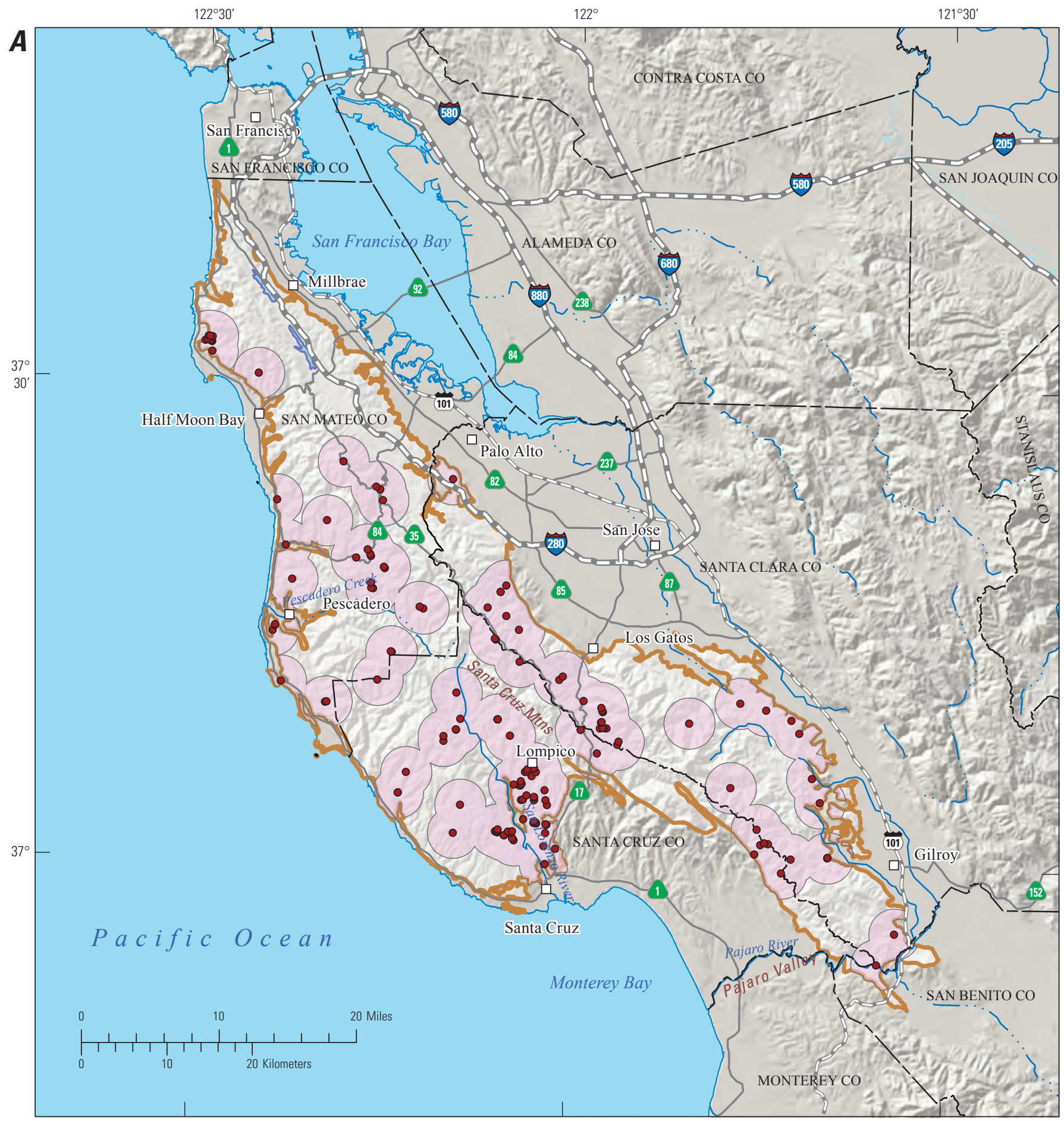

Shaded relief derived from U.S. Geological Survey National Elevation Dataset, 2006,

\section{EXPLANATION}

Albers Equal Area Conic Projection

Santa Cruz study area
Santa Cruz 3-kilometer buffer
- CDPH well

Figure 3. (A) Santa Cruz, $(B)$ San Gabriel, and $(C)$ Peninsular Ranges study areas showing the 3-kilometer buffers, locations of wells in the California Department of Public Health (CDPH) database, counties, major roads and cities, and hydrologic and topographic features, Hard Rock study unit, California GAMA Priority Basin Project. 


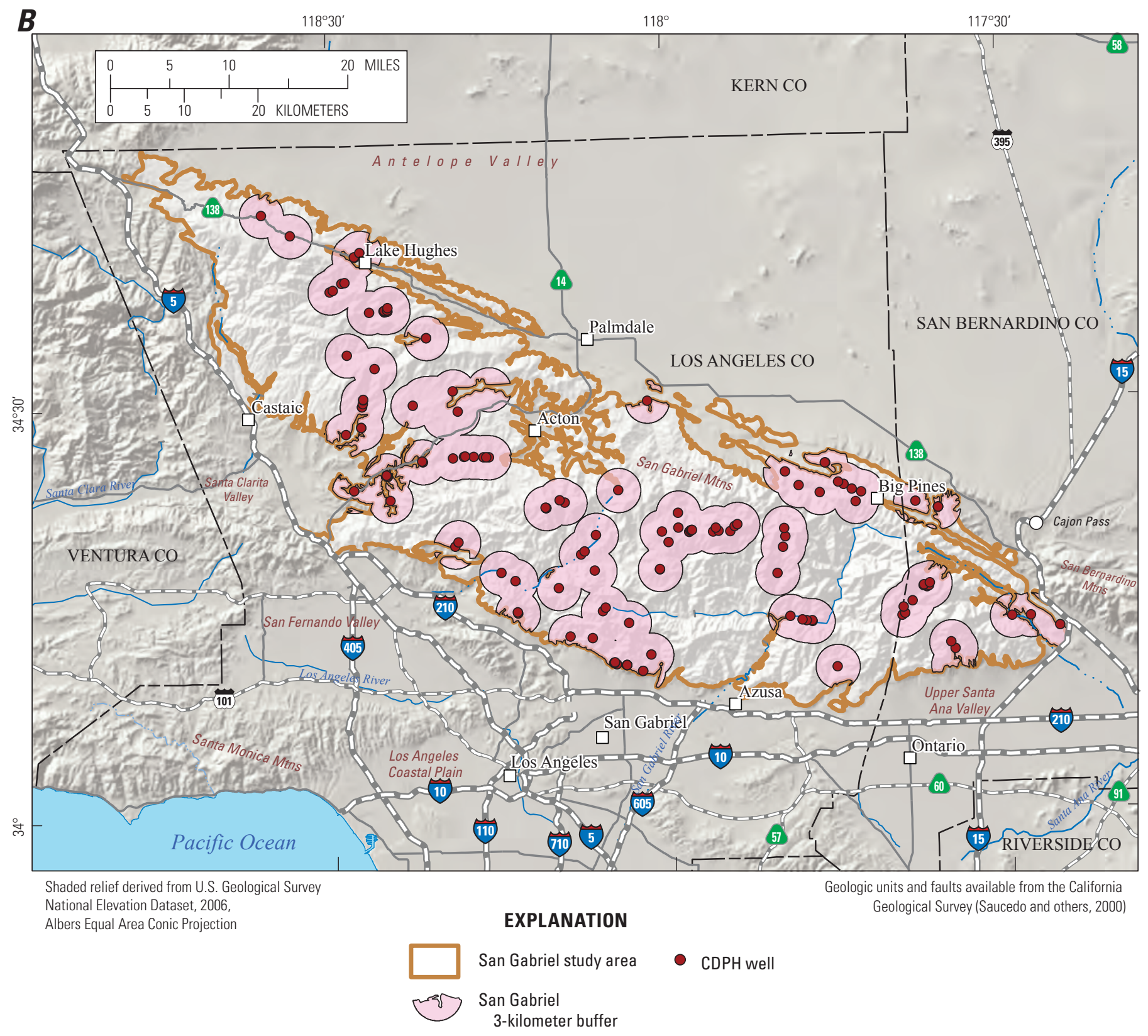

Figure 3. - Continued 


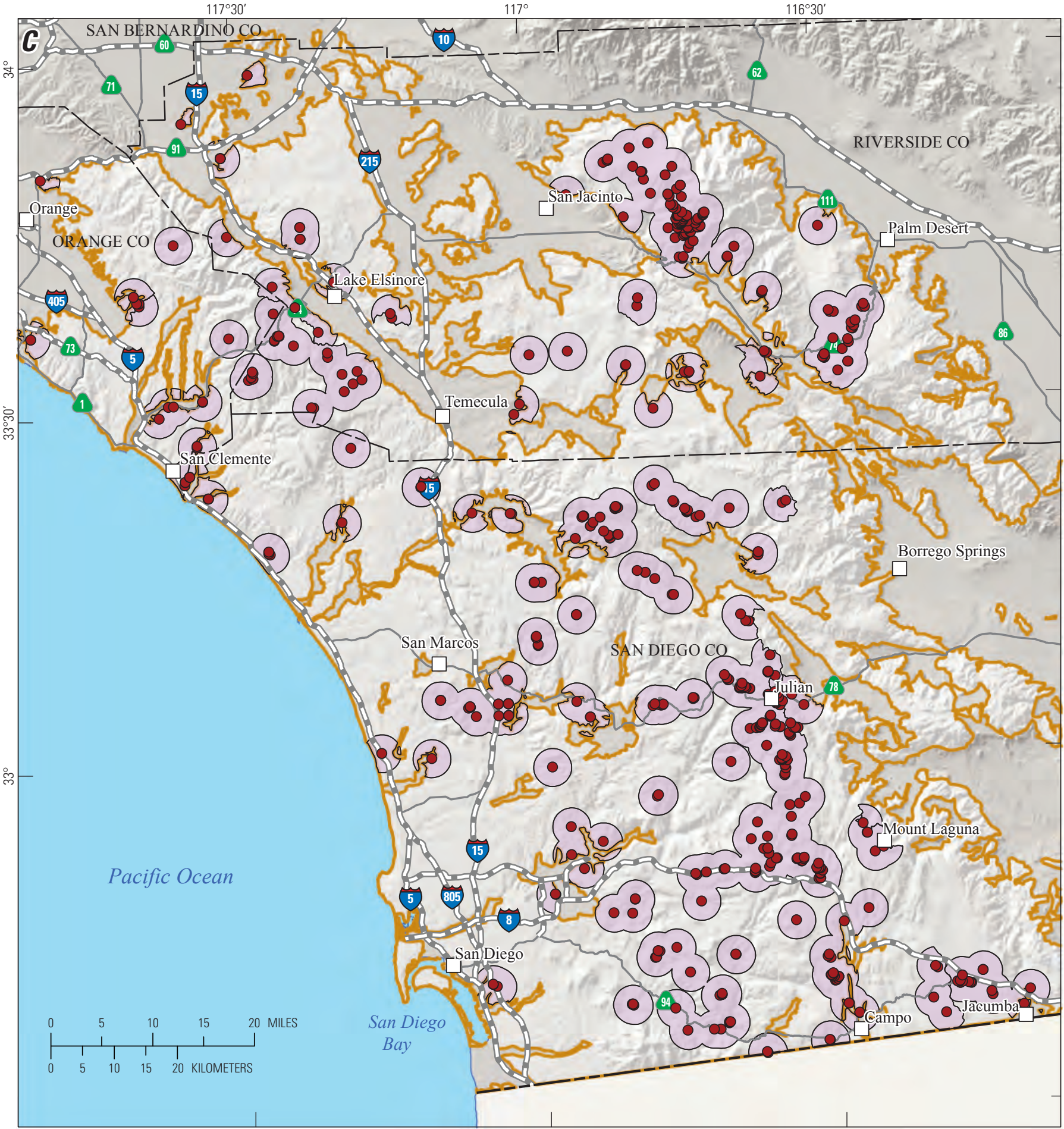

Shaded relief derived from U.S. Geological Survey National Elevation Dataset, 2006,

EXPLANATION

Albers Equal Area Conic Projection

Peninsular Ranges

3-kilometer buffer

Figure 3. - Continued 


\section{San Gabriel Study Area (SG)}

The San Gabriel study area of the Hard Rock study unit is located in the Transverse and Selected Peninsular Ranges hydrogeologic province (fig. 1) and includes parts of Los Angeles and San Bernardino Counties (fig. 3B). The study area contains the San Gabriel and Castaic Mountains, is approximately 85 miles in length and up to 20 miles wide, and is wedged between major fault zones (fig. $2 B$ ). Along the northeast flank, the SAFZ separates the mountains from the Antelope Valley. The study area is bounded in the east by the boundary of the San Gabriel and San Bernardino Mountains, known as the Cajon Pass area (California Department of Water Resources, 2004a). The Sierra Madre and San Gabriel faults meet on the southwest flank and extend northwest up to Piru Creek, almost connecting with the SAFZ. The study unit boundaries were defined primarily by the extent of Mesozoic granitic rocks, Mesozoic and older metamorphic rocks, and lithified Tertiary sedimentary rocks in the mountain block. Alluvial valleys within the mountain block were excluded. The alluvial valleys of the Santa Clarita Valley in the southwestern part of the mountain block were part of the GAMA-PBP Santa Clara River Valley study unit (Montrella and Belitz, 2009). The basins along the southern margin of the mountain block composed the GAMA-PBP San Fernando-San Gabriel (Land and Belitz, 2008) and Upper Santa Ana Watershed (Kent and Belitz, 2009) study units; the Antelope Valley and small basins in the SAFZ were included in the GAMA-PBP Antelope Valley study unit (Schmitt and others, 2009).

The SG study area consists primarily of granitic, plutonic rocks (Mesozoic through Precambrian) and schist (fig. $2 B$ ). Tertiary, lithified sediments and mafic volcanics are interstratified with schist in the Castaic Mountains located in the northwestern portion of the study area. Several streams, including the San Gabriel River, Castaic Creek, and Lytle Creek, cut through the mountains and flow outside the study area into local reservoirs or into rivers that travel south through the coastal valleys and eventually terminate in the Pacific Ocean (California Department of Water Resources, 2004c). The streams are the main sources of surface-water discharge for the study area. In some areas, water stored in fractured hard rock forms springs or recharges local waterbearing formations for nearby groundwater alluvial basins (California Department of Water Resources, 2004b).

The climate in the San Gabriel study area is characterized as Mediterranean, with warm, dry summers and cool, wet winters. The NCDC station located on Mount Wilson (fig. 2B) measured an average annual temperature of $55^{\circ} \mathrm{F}\left(13^{\circ} \mathrm{C}\right)$ for 2011; total annual precipitation was measured at about 14 inches (U.S. Department of Commerce, National Climatic Data Center, 2011). Precipitation primarily occurs as rain during the winter and early spring. Snow occurs in the winter months at the higher elevations. The highest peak in the San Gabriel Mountains is Mount San Antonio at an elevation of about 10,000 feet.

\section{Peninsular Ranges Study Area (PR)}

The Peninsular Ranges study area of the Hard Rock study unit is located in the southwest corner of California, in San Diego, Riverside, and Orange Counties (fig. 3C). The PR study area is primarily composed of the mountainous regions of the San Diego Drainages hydrogeologic province and includes portions of the Transverse and Selected Peninsular Ranges hydrogeologic province to the north and the Desert hydrogeologic province to the east (fig. 1).

The PR mountain ranges include the Santa Ana, San Jacinto, Palomar, Cuyamaca, and San Ysidro Mountains. The PR study area consists primarily of Mesozoic granitic rocks, which in some regions are intermixed with Santiago Peak volcanic rocks and gabbroic plutons (fig. $2 \mathrm{C}$ ). The Santiago Peak volcanics are metavolcanic, volcaniclastic, and sedimentary rocks located along the western zone of the Peninsular Ranges batholith, which extends south to Baja California (Tanaka and others, 1984). Toward the coast, the mountains give way to younger (Mesozoic to Tertiary) lithified sediments. The boundaries of the study area were defined by the extent of these igneous, metamorphic, and lithified sedimentary rocks; areas of Quaternary alluvium were excluded from the study area. Alluvial valleys within and surrounding the study area were included in the GAMA-PBP San Diego Drainages (Wright and others, 2005), Upper Santa Ana Watershed (Kent and Belitz, 2009), Los Angeles Coastal Plain (Mathany and others, 2008), and Coachella Valley (Goldrath and others, 2009) study units. The SAFZ branches out to two parallel systems in the area: the Elsinore and San Jacinto faults. Several rivers and creeks flow through the mountains and eventually terminate in the San Diego Bay in the southwest, the Pacific Ocean in the west, or the Salton Sea in the east. Major surface-water drainage features in the study area include the San Luis Rey, San Jacinto, Santa Margarita, San Diego, and Sweetwater Rivers and Santa Ysabel Creek.

The climate in the Peninsular Ranges study area is characterized as Mediterranean, with warm, dry summers and cool, wet winters. The NCDC station located at the Palomar Mountain Observatory (fig. 2C) measured an average annual temperature of $56^{\circ} \mathrm{F}\left(13^{\circ} \mathrm{C}\right)$ for 2011 ; total annual precipitation was about 25 inches (U.S. Department of Commerce, National Climatic Data Center, 2011). Precipitation primarily occurs as rain during the winter and early spring. Snow may occur in the winter months at the higher elevations. The highest peak in the Peninsular Ranges study area is San Jacinto Peak at an elevation of about 10,800 feet. 


\section{Methods}

Methods used for the GAMA-PBP were selected to achieve the following objectives: (1) design a sampling plan for suitable statistical representation; (2) collect samples in a consistent manner; (3) analyze samples by using proven and reliable laboratory methods; (4) assure the quality of the groundwater data; and (5) maintain data securely and with relevant documentation. The appendix contains detailed descriptions of the sample collection protocols, analytical methods, quality-assurance (QA) methods, and results of analyses of quality-control (QC) samples.

\section{Study Design}

Public-supply wells in the CDPH database located outside of CDWR-defined groundwater basins were mapped to determine the areas with relatively high well density. Wells that supply drinking water in the hard rock regions of the Klamath (Mathany and Belitz, 2014), North San Francisco Bay (Kulongoski and others, 2006), Sierra Nevada (Fram and Belitz, 2007; Ferrari and others, 2008; Fram and others, 2009; Shelton and others, 2010), Big Bear (Mathany and Belitz, 2013), and San Diego (Wright and others, 2005) study units have been sampled as part of previous GAMA-PBP studies. Of the remaining regions that are outside groundwater basins, three study areas with high densities of CDPH wells were selected: the Santa Cruz, San Gabriel, and Peninsular Ranges study areas.

CDPH wells were not distributed evenly over the study areas, and only the parts of the study areas in which groundwater resources are currently used for public drinkingwater supply were assessed in this study. The areas to be assessed were defined by drawing 3-kilometer $(\mathrm{km})$ (1.86-mile) radius circles around the locations of wells in the CDPH database, referred to as "buffers" (figs. 3A-C). Buffers around wells near the study area margins may not be circular because only areas within the study unit boundaries are included.

The collective area encompassed by the buffers was then divided into equal-area grid cells using a randomized gridbased method (Scott, 1990) (figs. 4A-C). The SC study area has a total buffer area of 450 square miles $\left(\mathrm{mi}^{2}\right)$, which was divided into 25 grid cells, each with an average area of $18 \mathrm{mi}^{2}$ (fig. 4A). The SG study area has a total buffer area of $546 \mathrm{mi}^{2}$, which was divided into 40 grid cells, each with an average area of $13 \mathrm{mi}^{2}$ (fig. 4B). The PR study area has a total buffer area of 1,405 $\mathrm{mi}^{2}$ which was divided into 41 grid cells, each with an average area of $34 \mathrm{mi}^{2}$ (fig. 4C). Because the total buffered area in each study area consists of many irregularlyshaped pieces and the sizes of the grid cells are much larger than the size of a buffer around an individual well, each cell generally includes several non-continuous areas.

Some of the sites listed in the CDPH database for the SC and SG study areas are springs. Springs are sites where groundwater naturally flows from below ground to above land surface, sometimes through a horizontal well bore. In the CDPH database, a spring with a horizontal well bore may have been given a name that identifies it as a well. All sites are referred to as wells in this report, unless the difference between a well and a spring is important to the discussion.

Sites were randomly selected to provide a statistically unbiased, spatially distributed assessment of the quality of the groundwater that is used for public drinking-water supply within each study area. The objective was to sample one CDPH well in each grid cell. If a grid cell contained more than one CDPH well, each well randomly was assigned a rank. The highest-ranking well that met basic sampling criteria (for example, sampling point located prior to treatment or capability to pump for several hours) was sampled. If a CDPH well was not accessible, then other types of wells, such as irrigation wells, domestic wells, or commercial wells, were considered for sampling. These "alternative" wells were identified from wells listed in the USGS National Water Information System database or by door-to-door canvassing. Wells with depths and screened intervals similar to those in CDPH wells in the area were selected. In this fashion, 1 well was selected for sampling in 94 of the 106 grid cells to provide a spatially distributed, randomized monitoring network. The remaining 12 cells contained no accessible wells.

The 94 wells sampled as part of the spatially distributed, randomized grid-cell network, hereinafter, are referred to as "grid wells." Eighteen additional, non-randomized wells (hereinafter referred to as "understanding wells") were selected to aid in the understanding of groundwater sources and flow through hard rock aquifers in the Hard Rock study unit. The understanding wells were not included in the statistical characterization of water quality in the Hard Rock study unit because inclusion of these wells would lead to the over-representation of some cells.

All 112 grid and understanding wells in the Hard Rock study unit were named by using the prefix "HR." The 94 grid wells sampled were named by using a suffix "SC," "SG," or "PR" depending on the study area, followed by a number representing the order in which they were sampled for each study area (figs. $4 A-C$ ). The 18 understanding wells sampled were named by adding "U" or "FP" to the study area abbreviation ("U" indicating "understanding" and "FP" indicating "flow path") followed by a number representing the order in which they were sampled for each study area. Flowpath wells are a subset of the understanding wells sampled for the PR study area and are located in the Sweetwater River watershed of San Diego County. 


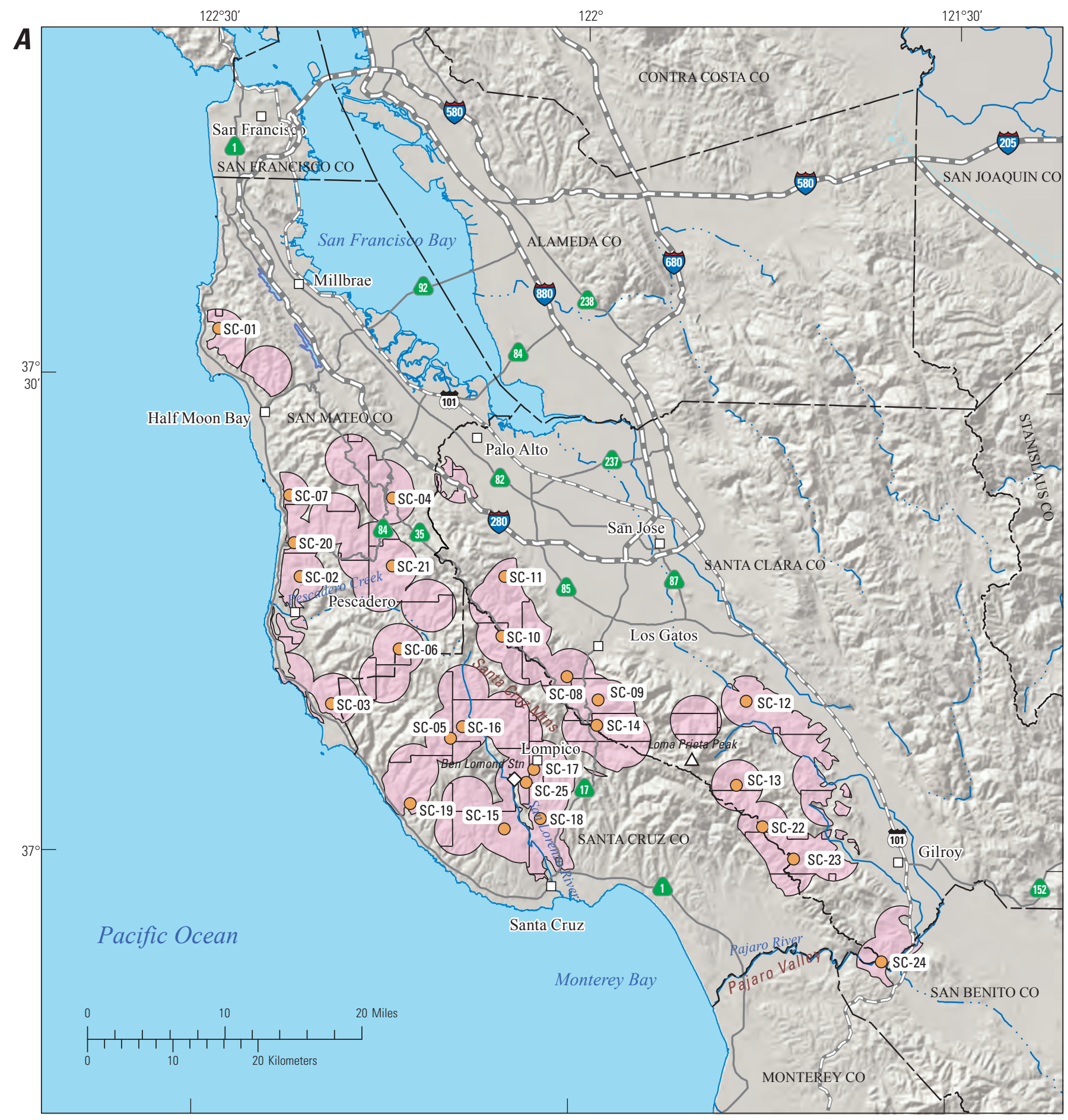

Shaded relief derived from U.S. Geological Survey National Elevation Dataset, 2006,

Albers Equal Area Conic Projection

\section{EXPLANATION}

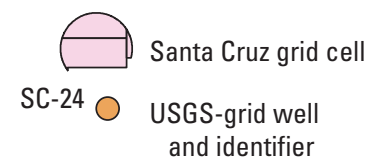

Figure 4. (A) Santa Cruz, $(B)$ San Gabriel, and $(C)$ Peninsular Ranges study areas showing the distribution of the grid cells, locations of sampled wells, major cities, and hydrologic and topographic features, Hard Rock study unit, California GAMA Priority Basin Project. 
The GAMA alphanumeric identification number for each well, along with the date sampled, well altitude, well type, and available well-construction information are shown in table 1. Groundwater samples were collected from March 28, 2011, through March 8, 2012. Grid wells included 83 production wells (74 CDPH, 4 domestic, 3 irrigation, 1 fire, and 1 unused) and 11 springs (all CDPH springs). Understanding wells included 15 production wells (5 CDPH, 6 domestic, and 4 irrigation) and 3 springs ( $1 \mathrm{CDPH}, 1$ domestic, and 1 spring used for bottling water).
Well locations were verified by using a global positioning system (GPS), 1:24,000-scale USGS topographic maps, well information in USGS and CDPH databases, and information provided by well owners, driller's logs, or other sources. Well locations and information were recorded by hand on field sheets, and electronically on field computers using the Alternate Place Entry (APE) program designed by the USGS. All information was verified and then uploaded into the USGS National Water Information System (NWIS). Well owner and water-use information is confidential.

\section{B}

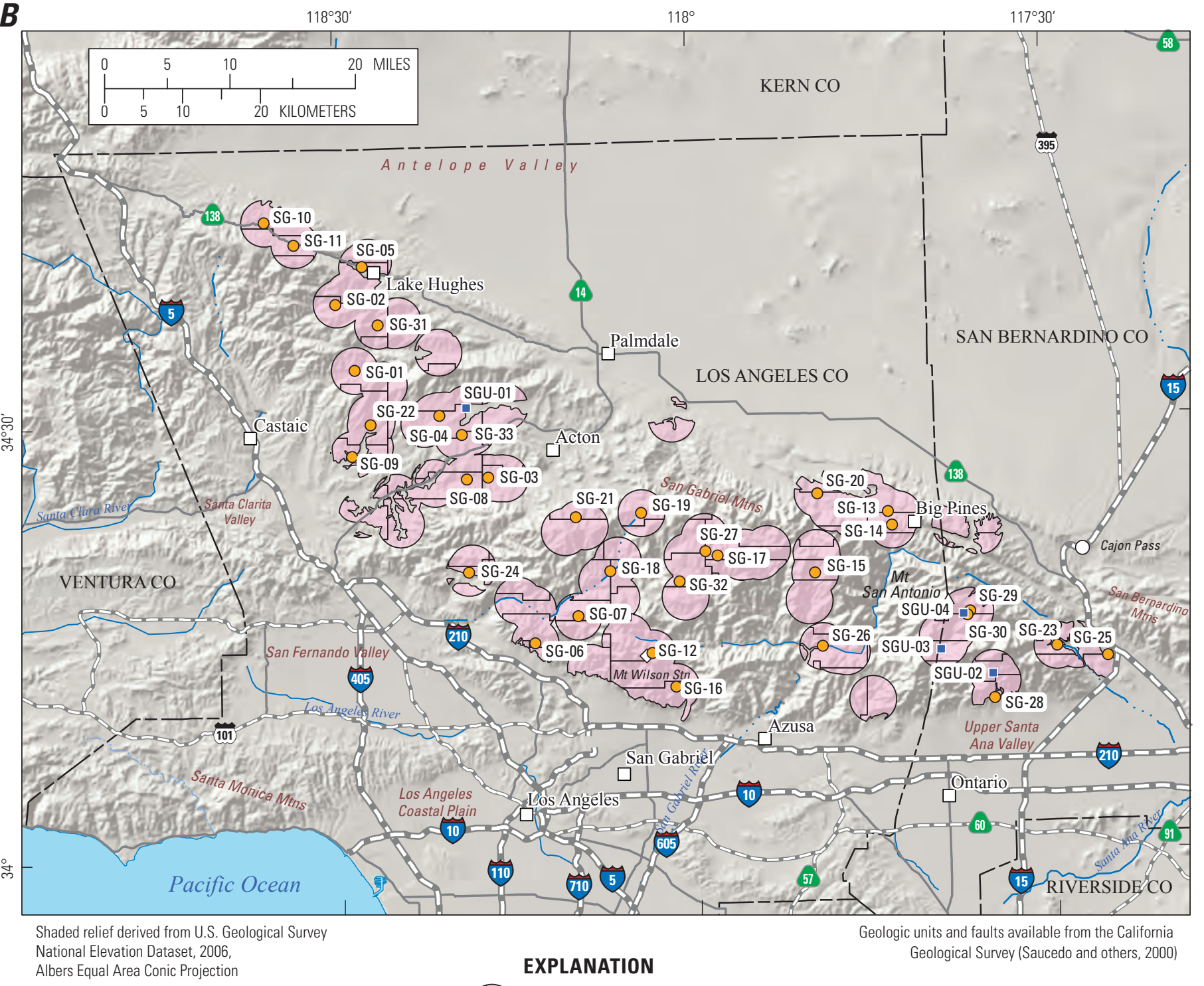

San Gabriel grid cell

SG-16 USGS-grid well and identifier

SGU-01 USGS-understanding well and identifier

Figure 4. - Continued 
C

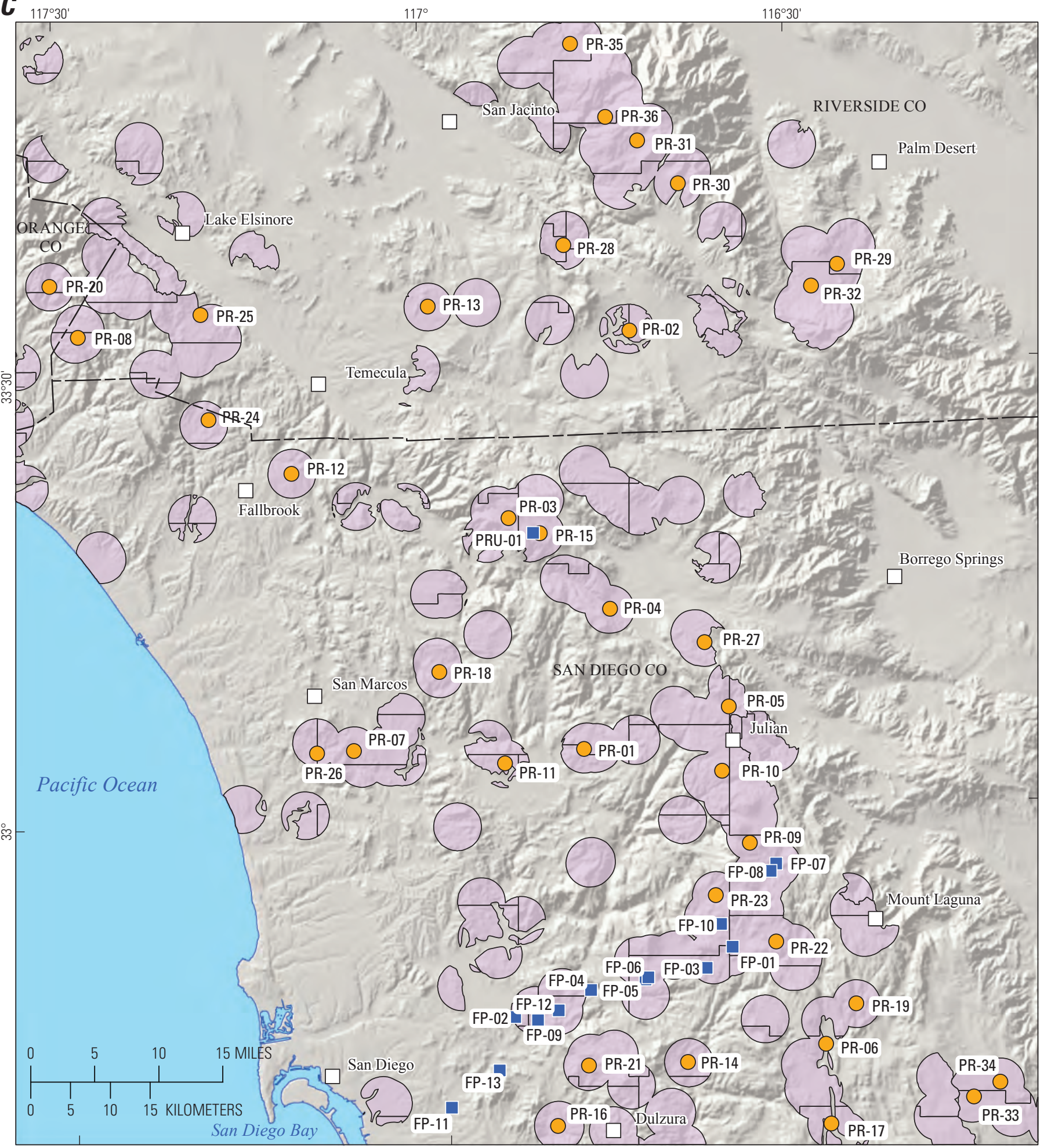

Shaded relief derived from U.S. Geological Survey

National Elevation Dataset, 2006,

Albers Equal Area Conic Projection

Peninsular Ranges
grid cell

EXPLANATION

PR-14 USGS-grid well PRU-01 — USGS-understanding well FP-09 $\square$ USGS-flow-path well and identifier and identifier and identifier

Figure 4. - Continued 


\section{Sample Collection and Analysis}

Samples were collected in accordance with the protocols established by the USGS National Water-Quality Assessment (NAWQA) Program (Koterba and others, 1995) and the USGS National Field Manual (NFM) (U.S. Geological Survey, variously dated). These sampling protocols were followed so that representative samples of groundwater were collected at each site and so that the samples were collected and handled in ways that minimized the potential for contamination. Use of the sampling protocols also allows comparison with data collected by the GAMA-PBP throughout California and with other USGS projects in California and the Nation.

All 112 wells in the Hard Rock study unit were sampled for a standard set of constituents (table 2); the 37 SG grid and understanding wells were also sampled for chromium species. Tables 3A-I list the compounds analyzed in each constituent class. Groundwater samples were analyzed for 85 VOCs (table 3A); 63 pesticides and pesticide degradates (table 3B); 1 constituent of special interest (table 3C); 24 trace elements (table 3D); 5 nutrients (table 3E); 9 major and minor ions, silica, total dissolved solids, and laboratory alkalinity (table 3F); arsenic, chromium, and iron species (table 3G); 5 isotopic tracers and 6 radioactive constituents, including tritium and carbon-14 abundance (table 3H); and stable isotope ratios of helium, 5 dissolved noble gases, and tritium (table 3I). The methods used for sample collection and analysis are described in the appendix section titled "Sample Collection and Analysis."

\section{Data Reporting}

The methods and conventions used for reporting the data are described in the appendix section titled "Data Reporting." Three water-quality indicators - alkalinity, $\mathrm{pH}$, and specific conductance - were measured in the field and at the National Water Quality Laboratory (NWQL), and four other constituents - tritium and total arsenic, chromium, and ironwere each measured by two different laboratories. Both sets of results for each constituent (except tritium) were reported, with preference given to one set according to the method of analysis; tritium does not have a preferred method; however, only one set of results was available at the time this report was published (see the appendix section titled "Constituents on Multiple Analytical Schedules").

\section{Comparative Benchmarks}

Concentrations of constituents detected in groundwater samples were compared with CDPH and USEPA regulatory and nonregulatory drinking-water health-based benchmarks and benchmarks established for aesthetic purposes (California Department of Public Health, 2012a,b; U.S. Environmental Protection Agency, 2009, 2012a,b). The water-quality data presented in this report are meant to characterize the quality of the untreated groundwater within the aquifers of the Hard Rock study unit and are not intended to represent the treated drinking water delivered to consumers by water purveyors. The chemical composition of treated drinking water may differ from untreated groundwater because treated drinking water may be subjected to disinfection, filtration, mixing with other waters, and (or) exposure to the atmosphere prior to its delivery to consumers. Comparisons of untreated groundwater to benchmarks are for illustrative purposes only and are not indicative of compliance or non-compliance with drinkingwater regulations. The benchmarks used for each constituent were selected in the following order of priority:

1. MCL-Maximum Contaminant Level. Legally enforceable standards that apply to public water systems and are designed to protect public health by limiting the levels of contaminants in drinking water. MCLs established by the USEPA are the minimum standards with which States are required to comply, and individual States may choose to set more stringent standards. The CDPH has established MCLs for additional constituents not regulated by the USEPA, as well as lowered the benchmark concentrations for a number of constituents with MCLs established by the USEPA. In this report, a benchmark set by the USEPA and adopted by the CDPH is labeled "MCL-US," and one set by the CDPH that is more stringent than the MCL-US is labeled "MCL-CA." Well owners are notified when constituents are detected at concentrations greater than an MCL-US or MCL-CA benchmark in samples collected for the GAMA-PBP, but these detections do not constitute violations of $\mathrm{CDPH}$ regulations.

2. AL-Action Level. Legally enforceable standards that apply to public water systems and are designed to protect public health by limiting the levels of copper and lead in drinking water. Detections of copper or lead greater than the action-level benchmarks trigger requirements for mandatory water treatment to reduce the corrosiveness of water to water pipes. The action levels established by the USEPA and CDPH are the same; thus, these benchmarks are labeled "AL-US" in this report.

3. SMCL-Secondary Maximum Contaminant Level. Non-enforceable standards applied to constituents that affect the aesthetic qualities of drinking water, such as taste, odor, and color, or the technical qualities of drinking water, such as scaling and staining. Both the USEPA and CDPH define SMCLs, but unlike MCLs, SMCLs established by the CDPH are not required to be at least as stringent as those established by the USEPA. SMCLs established by the CDPH are used in this report (SMCL-CA) for all constituents that have SMCL-CA values. The SMCL established by the USEPA (SMCL-US) is used for $\mathrm{pH}$ because no SMCL-CA has been defined. 
4. NL-Notification Level. Health-based standards established by the CDPH (NL-CA) for some of the constituents in drinking water that lack MCLs. If a constituent is detected in drinking water at concentrations greater than its NL-CA, California State law requires timely notification of local governing bodies and recommends consumer notification.

5. HAL-Lifetime Health Advisory Level. The maximum concentration of a constituent at which its presence in drinking water is not expected to cause any adverse carcinogenic effects for a lifetime of exposure. HALs are established by the USEPA (HAL-US) and are calculated assuming consumption of 2 liters (1 quart) of water per day over a 70 -year lifetime by a 70-kilogram (154-pound) adult and that 20 percent of a person's exposure comes from drinking water.

6. RSD5-Risk-Specific Dose. The concentration of a constituent in drinking water corresponding to an excess estimated lifetime cancer risk of 1 in 100,000. RSD5 is an acronym for risk-specific dose at the $10^{-5}$ risk level $\left(10^{-5}\right.$ equals $\left.1 / 100,000\right)$. RSD5s are calculated by dividing the $10^{-4}$ cancer-risk concentration established by the USEPA by 10 (RSD5-US).

Note that using this hierarchy to select the comparative benchmark for a constituent with more than one type of established benchmark will not necessarily result in selection of the benchmark with the lowest concentration. For example, for zinc the SMCL-CA is 5,000 micrograms per liter $(\mu \mathrm{g} / \mathrm{L})$ and the HAL-US is $2,000 \mu \mathrm{g} / \mathrm{L}$, but the comparative benchmark selected by this hierarchy is the SMCL-CA. The comparative benchmarks used in this report are listed in tables 3A-I for all constituents and in tables 4-14 for constituents detected in groundwater samples from the Hard Rock study unit. Established benchmarks are not available for all constituents analyzed for this study. Detections of constituents at concentrations greater than the selected comparative benchmarks are marked with asterisks $(*)$ in tables $4,8,9,10,11$, and 14 .

\section{Quality-Assurance Methods}

The QA methods used for this study followed the protocols used by the NAWQA Program (Koterba and others, 1995) and described in the NFM (U.S. Geological Survey, variously dated). The QA plan followed by the NWQL, the primary laboratory used to analyze samples for this study, is described by Stevenson (2013). QC samples collected in the Hard Rock study unit were blanks, replicates, and matrix and surrogate spikes. QC samples were collected to evaluate potential contamination, as well as bias and variability of the data that may have resulted from sample collection, processing, storage, transportation, and laboratory analysis. QA/QC procedures and results are described in the appendix section titled "Quality-Assurance and Quality-Control Methods and Results" and are summarized in the following section.

\section{Water-Quality Results}

\section{Quality-Control Results}

Results of QC analyses (blanks, replicates, and matrix and surrogate spikes) were used to evaluate the quality of the data for the groundwater samples. On the basis of detections in field blanks collected for this and for previous GAMA-PBP study units, as well as a review of available QA/QC information, reporting levels in this study unit were raised from the USGS NWQL levels for three volatile organic compounds (1,2,4-trimethylbenzene, carbon disulfide, and toluene), eight trace elements (copper, iron, lead, manganese, molybdenum, nickel, tungsten, and zinc), and three nutrients (ammonia, nitrite, and nitrite plus nitrate). The raised reporting levels are referred to as "study reporting levels" (SRLs). Detections of the 14 constituents with concentrations less than their SRLs were coded with a less than or equal to $(\leq)$ symbol in this report and the NWIS database. Additionally, all groundwater detections for three VOCs (2-butanone, acetone, and tetrahydrofuran) and all results for one trace element (cobalt) were coded as "reviewed and rejected." For blank results for this study unit and references for additional information, see table $\mathrm{A} 3$ and the appendix section titled "Detections in Blanks and Application of SRLs."

Results from the replicates confirm that the procedures used to collect and analyze the samples were consistent. Variability for about 98 percent of the replicate pairs for constituents detected in samples was within acceptable limits (tables A4A-C). The criteria for acceptable replication are described in the appendix section titled "Methods of Assessment of Replicate Samples." Of the 148 organic constituents analyzed, 11 pesticides or pesticide degradates had median matrix-spike recoveries lower than the acceptable limit of 70 percent, and 1 pesticide had a median recovery greater than the acceptable limit of 130 percent (tables 3A-B and $\mathrm{A} 5 \mathrm{~A}-\mathrm{B})$. The constituents with low recoveries might not have been detected in some samples if they were present in the samples at concentrations near laboratory reporting levels (LRLs). Constituents with high recoveries may indicate that reported values could be greater than what is in the sample. The QC results are described in the appendix section titled "Matrix-Spike Recoveries."

\section{Groundwater-Quality Data}

Results from analyses of untreated groundwater samples from the Hard Rock study unit are presented in tables 4-14. These results are separated into the three study areas (SC, SG, and PR) that make up the Hard Rock study unit. Groundwater samples collected in the Hard Rock study unit were analyzed for 209 unique constituents; 115 of those constituents were not detected in any of the samples, and 92 constituents were detected at least once. The results for stable isotopes of dissolved boron, isotope ratios for dissolved strontium, and 
tritium (analyzed at the LLNL) were not available at the time this report was written; they will be included in subsequent publications. Tritium data analyzed at the USGS Stable Isotope and Tritium Laboratory (SITL) are presented in this report.

For organic and special-interest constituents, the results listed in the tables include only those constituents that were detected, and the following summary statistics are presented only for the grid wells: the number of wells at which each constituent was detected, the frequency at which it was detected (in relation to the number of grid wells in the study unit), and the total number of constituents detected at each well. For the inorganic, isotopic, and radioactive constituents, the tables include all of the constituents that were analyzed, as all constituents were detected in at least one well sample.

Water-quality indicators measured in the field and at the NWQL are included in table 4. The results of groundwater analyses, organized by constituent class, are presented in tables 5-14:

- Organic constituents

- Volatile organic compounds (table 5)

- Pesticides and pesticide degradates (table 6)

- Perchlorate (table 7)

- Inorganic constituents

- Trace elements (table 8)

- Nutrients (table 9)

- Major and minor ions, silica, and total dissolved solids (table 10)

- Arsenic, chromium, and iron species (table 11)

- Isotope tracers (table 12)

- Dissolved noble gases (table 13)

- Radioactive constituents

- Radon-222 (table 14A)

- Gross alpha and gross beta radioactivity (table 14B)

- Uranium-234, -235, and -238 (table 14C)

\section{Water-Quality Indicators}

Field measurements of dissolved oxygen and water temperature and field and laboratory measurements of $\mathrm{pH}$, specific conductance, and alkalinity are presented in table 4. Bicarbonate and carbonate concentrations were calculated from the $\mathrm{pH}$ and alkalinity results. Dissolved oxygen and alkalinity are used as indicators of natural processes that affect water chemistry. The $\mathrm{pH}$ value indicates the acidity or basicity of the water. Specific conductance is the measure of electrical conductivity of the water and is proportional to amount of total dissolved solids (TDS) in the water.

Field $\mathrm{pH}$ values were outside of the SMCL-US range (6.5 to 8.5) for 14 of the 94 Hard Rock study unit grid-well samples: 10 well samples had field $\mathrm{pH}$ values less than 6.5; 4 well samples had field $\mathrm{pH}$ values greater than 8.5 (table 4). One understanding-well sample in the PR study area had a field $\mathrm{pH}$ value greater than 8.5 . Low $\mathrm{pH}$ in water may contribute to corrosion of pipes, and high $\mathrm{pH}$ in water may contribute to scaling. Laboratory $\mathrm{pH}$ values may differ from field $\mathrm{pH}$ values because the $\mathrm{pH}$ of groundwater may change upon removal from the ambient environment and exposure to the atmosphere.

Field specific-conductance values were greater than the recommended SMCL-CA of 900 microsiemens per centimeter at 25 degrees Celsius $\left(\mu \mathrm{S} / \mathrm{cm}\right.$ at $\left.25^{\circ} \mathrm{C}\right)$ for 18 of the gridwell samples, three of which were also greater than the upper benchmark of $1,600 \mu \mathrm{S} / \mathrm{cm}$ at $25^{\circ} \mathrm{C}$ (table 4). Samples from six understanding wells in the PR study area were greater than the recommended SMCL-CA, three of which were also greater than the upper SMCL-CA.

\section{Organic Constituents}

VOCs are present in paints, solvents, fuels, fuel additives, refrigerants, fumigants, and disinfected water, and are characterized by their tendency to evaporate. VOCs generally persist longer in groundwater than in surface water because groundwater is isolated from the atmosphere. Of the 85 VOCs analyzed for the Hard Rock study unit, 19 were detected in grid-well samples (table 5). All concentrations were less than health-based benchmarks. One or more VOCs were detected in 41 of the 94 grid-well samples (44 percent detection frequency). The trihalomethane chloroform was the most frequently detected VOC in the study unit. Chloroform is among the most commonly detected VOCs in groundwater nationally, and its source is attributed, in part, to the recycling of chlorinated waters to aquifers (Zogorski and others,

2006). The detection frequency of chloroform was greater than 10 percent. Other than chloroform, no other VOCs had detection frequencies greater than 10 percent in the grid wells for all three study areas; however, two VOCs had detection frequencies greater than 10 percent in at least one study area. The detection frequency of the solvent 1,1-dichloroethene (1,1-DCE) was greater than 10 percent in samples from the SC study area. The detection frequency of the gasoline oxygenate methyl tert-butyl ether (MTBE) was greater than 10 percent in the PR study area. One or more VOCs were detected in 7 of the 18 understanding-well samples, all of which were from the PR study area.

Pesticides include herbicides, insecticides, and fungicides and are used to control weeds, insects, fungi, and other pests in agricultural, urban, and suburban settings. Of the 63 pesticides and pesticide degradates analyzed for the Hard Rock study unit, 14 were detected in grid-well samples (table 6). All 
concentrations were less than health-based benchmarks. One or more pesticides were detected in 17 of the 93 grid-well samples (18 percent detection frequency). There were no pesticide results for one grid well (HR-PR-01) because the sample was ruined during analysis. None of the pesticides had detection frequencies greater than 10 percent in the study unit as a whole; however, the herbicide simazine and the herbicide degradate deethylatrazine had detection frequencies greater than 10 percent in the PR study area. Simazine and deethylatrazine are among the most commonly detected pesticide compounds in groundwater nationally (Gilliom and others, 2006). One or more pesticides were detected in 4 of the 18 understanding wells, all of which were in the PR study area.

\section{Constituent of Special Interest}

Perchlorate was classified as a special-interest constituent because at the inception of the GAMA-PBP in 2003, perchlorate had recently been detected in public-supply wells in several areas of the State and the CDPH was evaluating whether or not an MCL-CA should be established (California Department of Public Health, 2012c). The MCL-CA of $6 \mu \mathrm{g} / \mathrm{L}$ was promulgated in 2007. Perchlorate was detected in 45 of 94 grid-well samples (48 percent detection frequency) and in 9 of the understanding-well samples (table 7). The three study areas of the Hard Rock study unit had different detection frequencies of perchlorate: 36 percent for SC, 55 percent for $\mathrm{SG}$, and 50 percent for PR. Perchlorate was not detected at concentrations greater than the MCL-CA of $6 \mu \mathrm{g} / \mathrm{L}$ at any of the grid or understanding wells in the Hard Rock study unit.

\section{Inorganic Constituents}

Unlike the organic and special-interest constituents, most of the inorganic constituents generally are naturally present in groundwater, although their concentrations may be influenced by human activities.

Seventeen of the trace elements (table 3D) and 1 of the major and minor ions (table 3F) analyzed and detected in the Hard Rock study unit have regulatory or nonregulatory healthbased benchmarks. Of these 18 constituents with health-based benchmarks, concentrations were less than their respective benchmarks in all grid-well detections for 13 constituents (tables 8,10 ). The remaining five inorganic constituents (arsenic, boron, molybdenum, uranium, and fluoride) were each detected at concentrations greater than their health-based benchmarks in one or more grid-well samples.

Arsenic concentrations greater than the MCL-US of $10 \mu \mathrm{g} / \mathrm{L}$ were detected in grid-well samples from two wells in the SC study area and one well in the SG study area (table 8). Boron concentrations greater than the NL-CA of $1,000 \mu \mathrm{g} / \mathrm{L}$ were detected in grid-well samples from two wells in the SC study area and one well in the SG study area. Molybdenum concentrations greater than the HAL-US of $40 \mu \mathrm{g} / \mathrm{L}$ were detected in grid-well samples from one well in the SC study area and one well in the SG study area. Fluoride concentrations greater than the MCL-CA of 2 milligrams per liter $(\mathrm{mg} / \mathrm{L})$ were detected in grid-well samples from one well in the SC study area and two wells in the SG study area (table 10). Uranium concentrations are discussed in the "Radioactive Constituents and Uranium" section.

Arsenic, strontium, and fluoride were detected at concentrations greater than their health-based benchmarks in one or more understanding wells (tables 8, 10). Arsenic concentrations greater than the benchmark level were detected in two understanding-well samples. A strontium concentration greater than the HAL-US of $4,000 \mu \mathrm{g} / \mathrm{L}$ was detected in one understanding-well sample. A fluoride concentration greater than the benchmark level was detected in one understandingwell sample.

Nutrients (nitrogen and phosphorus) can affect biological activity in aquifers and in surface-water bodies that receive groundwater discharge. Inorganic nitrogen may be present in the form of ammonia, nitrite, or nitrate, depending on the oxidation-reduction state of the groundwater. High concentrations of nitrate can adversely affect human health, particularly the health of infants (California Department of Public Health, 2012d). Most concentrations of ammonia, nitrite, and nitrate in samples from the 94 Hard Rock study unit grid wells were less than health-based benchmarks (table 9). Exceptions are detections of nitrite plus nitrate (as nitrogen) at concentrations greater than the MCL-US of $10 \mathrm{mg} / \mathrm{L}$ in grid-well samples from two wells in the PR study area. Of the 18 understanding-well samples, nitrite plus nitrate concentrations were greater than the benchmark in 4 samples, all of which came from flow-path wells in the PR study area.

The levels of certain trace elements, major-ion composition, and TDS content in groundwater affect the aesthetic properties of water, such as taste, color, and odor, and the technical properties of water, such as scaling and staining. Although there are no adverse health effects directly associated with these properties, they may reduce consumer satisfaction with the water or may have economic effects. The CDPH has established non-enforceable benchmarks (SMCL-CAs) for iron, manganese, silver, zinc, chloride, sulfate, and TDS that are based on aesthetic properties rather than on human-health concerns. Iron and manganese are trace elements whose concentrations are affected by the oxidationreduction state of the groundwater. Precipitation of minerals containing iron or manganese may cause orange, brown, or black staining of surfaces.

Iron concentrations greater than the SMCL-CA of $300 \mu \mathrm{g} / \mathrm{L}$ were detected in grid-well samples from eight wells in the SC study area, three wells in the SG study area, and eight wells in the PR study area (table 8). Manganese 
concentrations greater than the SMCL-CA of $50 \mu \mathrm{g} / \mathrm{L}$ were detected in grid-well samples from 13 wells in the SC study area, 3 wells in the SG study area, and 11 wells in the PR study area. Chloride concentrations were greater than the recommended SMCL-CA of $250 \mathrm{mg} / \mathrm{L}$ in grid-well samples from one well in the SC study area and two wells in the PR study area; one of the PR grid-well samples also had a concentration greater than the upper SMCL-CA of $500 \mathrm{mg} / \mathrm{L}$ (table 10). Sulfate concentrations were greater than the recommended SMCL-CA of $250 \mathrm{mg} / \mathrm{L}$ for grid-well samples from one well in the SC study area and three wells in the PR study area; none of these concentrations were greater than the upper SMCL-CA of $500 \mathrm{mg} / \mathrm{L}$. TDS concentrations were greater than the recommended SMCL-CA of $500 \mathrm{mg} / \mathrm{L}$ for grid-well samples from 8 wells in the SC study area, 4 wells in the SG study area, and 15 wells in the PR study area; one of the SC and two of the PR grid-well samples had TDS concentrations greater than the upper SMCL-CA of $1,000 \mathrm{mg} / \mathrm{L}$.

Iron was detected at concentrations greater than the benchmark level in two understanding-well samples, and manganese was detected at concentrations greater than the benchmark level in eight understanding-well samples (table 8). Chloride was detected at concentrations greater than the recommended benchmark in four understandingwell samples; two of these concentrations were greater than the upper benchmark (table 10). Sulfate was detected at concentrations greater than the recommended benchmark in two understanding-well samples. TDS was detected at concentrations greater than the recommended benchmark in nine understanding-well samples; three of these concentrations were greater than the upper benchmark.

\section{Geochemical and Age-Dating Tracers}

The geochemical and age-dating tracers may be used to help interpret hydrologic processes affecting groundwater quality (Clark and Fritz, 1997). Arsenic, chromium, and iron occur as different species depending on the oxidationreduction state of the groundwater. The oxidized and reduced species have different solubilities in groundwater and may have different effects on human health. The relative proportions of the oxidized and reduced species of each element can be used to aid in interpretation of the oxidationreduction conditions of the aquifer, which affect the mobility of many constituents. Concentrations of total arsenic, chromium, and iron and the dissolved concentration of either the reduced or the oxidized species of these elements are reported in table 11.
Stable-isotope ratios of hydrogen and oxygen in water reflect the altitude, latitude, and temperature of precipitation and also the extent of evaporation of the water in surfacewater bodies or soils prior to infiltration into the aquifer. These data aid in the interpretation of the sources of groundwater recharge. Results for stable isotopes of hydrogen, oxygen, and carbon are reported in table 12 (see appendix section "Notation" for reporting methods for stable isotope data).

Carbon-14 is a radioactive isotope used for age-dating (time since recharge). Low levels of carbon-14 are produced continuously by interaction of cosmic radiation with the Earth's atmosphere and are incorporated into atmospheric carbon dioxide. Carbon dioxide dissolves in precipitation, surface water, and groundwater exposed to the atmosphere, thereby entering the hydrologic cycle. Because carbon-14 decays with a half-life of approximately 5,700 years, low activities of carbon-14, relative to modern values, generally indicate a presence of groundwater that is several thousands of years old or more. Carbon-14 results, as percent modern carbon, are reported in table 12 .

Tritium activities and dissolved noble gases also provide information about the age of groundwater. Tritium is a shortlived radioactive isotope of hydrogen that is incorporated into the water molecule. Low levels of tritium are produced continuously by interaction of cosmic radiation with the Earth's atmosphere, and a large amount of tritium was produced as a result of atmospheric testing of nuclear weapons between 1952 and 1963. Thus, concentrations of tritium greater than background levels generally indicate the presence of water recharged after the early 1950s. Helium isotope ratios are used in conjunction with tritium concentrations to estimate ages for young groundwater. Of the isotope-tracer constituents analyzed for this study, tritium is the only one with a healthbased benchmark. All measured tritium activities in samples from the Hard Rock study unit were less than 1/1,000 of the MCL-CA benchmark (table 12).

Gases dissolve in water that is in contact with the atmosphere, and the solubilities of the different gas species vary with temperature. Concentrations of dissolved noble gases are used to estimate the conditions of groundwater recharge, particularly the temperature of the water at the time of recharge. Results for analyses of dissolved noble gases (argon, helium, krypton, neon, and xenon) are reported in table 13. These results are stored in a database separate from the NWIS database and maintained by GAMA-PBP project staff. 


\section{Radioactive Constituents and Uranium}

Radioactivity is the release of energy or energetic particles during changes in the structure of the nucleus of an atom. Most of the radioactivity in groundwater comes from decay of naturally occurring isotopes of uranium and thorium that can be present in minerals in the sediments or fractured rocks of an aquifer. Uranium and thorium decay in a series of steps eventually forming stable isotopes of lead (Soddy, 1913; Faure and Mensing, 2005). In each step in the decay series, one radioactive element turns into a different radioactive element by emitting an alpha or a beta particle from its nucleus. The number of particles emitted is equal to the number of atoms decaying. Concentrations of radioactive constituents in groundwater are expressed in picocuries per liter; 1 picocurie per liter $(\mathrm{pCi} / \mathrm{L})$ is approximately equal to two atoms decaying per minute.

The alpha and beta particles emitted during radioactive decay may be hazardous to human health because these energetic particles may damage cells. Radiation damage to cell DNA may increase the risk of getting cancer (U.S. Environmental Protection Agency, 2013). For that reason, MCLs were set by the USEPA and CDPH to regulate concentrations of gross alpha and beta radioactivity and uranium in drinking water. The MCL-US for gross alpha radioactivity applies to adjusted gross alpha radioactivity, which is equal to measured gross alpha activity minus uranium activity. The proposed MCL-US for radon-222 will apply if the State or local water agency has an approved multimedia mitigation program to address radon levels in indoor air (U.S. Environmental Protection Agency, 2012b).

Of the 112 wells in the Hard Rock study unit, samples from 105 wells were analyzed for radon-222, samples from 110 wells were analyzed for gross alpha and beta activities, and samples from 29 wells were analyzed for uranium isotopes (uranium-234, uranium-235, and uranium-238). Radon-222 activities were less than the proposed MCL-US of $4,000 \mathrm{pCi} / \mathrm{L}$ in most of the sampled wells; exceptions were grid-well samples from two wells in the SG study area and three wells in the PR study area (table 14A). Gross alpha activity was greater than the MCL-US of $15 \mathrm{pCi} / \mathrm{L}$ in gridwell samples from four wells in the SG study area and seven wells in the PR study area (table 14B). Two of the SG and four of the PR grid-well samples having unadjusted gross alpha activity greater than the benchmark also had total uranium concentrations greater than the MCL-US of $30 \mu \mathrm{g} / \mathrm{L}$; the same wells plus one PR grid-well sample had uranium activities greater than the MCL-CA of $20 \mathrm{pCi} / \mathrm{L}$ (tables 8, 14C).

Radon-222 was greater than the proposed MCL-US in five understanding-well samples (table 14A). Gross alpha activities were greater than the benchmark in seven understanding-well samples. One of these samples also had gross beta activity (30-day count) greater than the MCL-CA of $50 \mathrm{pCi} / \mathrm{L}$ (table 14B). Five of the seven understandingwell samples having unadjusted gross alpha activity greater than the MCL-US also had total uranium concentrations greater than the MCL-US and uranium activities greater than the MCL-CA. One additional understanding-well sample had uranium concentration and activity greater than the benchmarks, but gross alpha and beta activities were not analyzed for this sample (tables 8, 14C).

\section{Future Work}

Subsequent reports for the Hard Rock study unit will be focused on assessment of the data presented in this report by using a variety of statistical, qualitative, and quantitative approaches to evaluate the natural and human factors affecting groundwater quality in the Hard Rock study unit. Water-quality data contained in the CDPH databases will be compiled, evaluated, and used in combination with the data that are presented in this report.

\section{Summary}

Groundwater quality in the 2,400-square-mile Santa Cruz, San Gabriel, and Peninsular Ranges Hard Rock Aquifers (Hard Rock) study unit was investigated by the U.S. Geological Survey (USGS) from March 2011 through March 2012, as part of the California State Water Resources Control Board (SWRCB) Groundwater Ambient Monitoring and Assessment (GAMA) Program's Priority Basin Project (PBP). The GAMA Program was created to provide a comprehensive baseline of groundwater quality in the State of California. The GAMA-PBP was created as a result of the Groundwater Quality Monitoring Act of 2001 (Sections 10780-10782.3 of the California Water Code, Assembly Bill 599) to assess and monitor the quality of groundwater. The GAMA-PBP is being conducted by the USGS in cooperation with the SWRCB and Lawrence Livermore National Laboratory.

The GAMA Hard Rock study was designed to provide a spatially unbiased assessment of untreated-groundwater quality within the primary aquifer system and to facilitate statistically consistent comparisons of untreated groundwater quality throughout California. The primary aquifer system is defined as the depth intervals of the wells listed in the California Department of Public Health (CDPH) database for the Hard Rock study unit. The quality of groundwater in the primary aquifer system may differ from that in shallow or deeper water-bearing zones; shallow groundwater may be more vulnerable to surficial contamination.

This study did not attempt to evaluate the quality of water delivered to consumers; after withdrawal from the ground, water typically is treated, disinfected, and blended with other waters to maintain acceptable water quality. Regulatory benchmarks apply to treated water that is served to the consumer, not to untreated groundwater. However, to provide some context for the results, concentrations of 
constituents measured in the untreated groundwater samples were compared with regulatory and nonregulatory healthbased benchmarks established by the U.S. Environmental Protection Agency (USEPA) and the CDPH and nonregulatory benchmarks established for aesthetic concerns by the CDPH.

The Hard Rock study unit is located within the Southern Coast Ranges, Transverse and Selected Peninsular Ranges, San Diego Drainages, and Desert hydrogeologic provinces. The study unit includes areas outside of the groundwater basins defined by the California Department of Water Resources. The Hard Rock study included assessment of the groundwater quality from 112 wells and springs in 3 study areas (the Santa Cruz, San Gabriel, and Peninsular Ranges) in San Mateo, Santa Clara, Santa Cruz, San Benito, Los Angeles, Orange, Riverside, San Bernardino, and San Diego Counties. All sites are referred to as wells in this report unless the difference between a well and a spring is important to the discussion. Ninety-four sites were selected by using a randomized grid approach to achieve a statistically unbiased representation of groundwater used for public drinkingwater supplies (grid wells): 25 in the Santa Cruz (SC) study area, 33 in the San Gabriel (SG) study area, and 36 in the Peninsular Ranges (PR) study area. Eighteen additional wells were selected to aid in the evaluation of water-quality issues (understanding wells).

Groundwater samples were analyzed for field waterquality indicators, organic constituents, one special-interest constituent, inorganic constituents, geochemical and age-dating tracers, and radioactive constituents. In total, 209 different constituents and water-quality indicators were measured in this study. This report describes the sampling, analytical, and quality-assurance methods used in the study and presents the results of the chemical analyses of the groundwater samples.

Three types of quality-control samples (blanks, replicates, and matrix spikes) were collected at approximately 10 percent of the wells in the Hard Rock study unit, and the results for these samples were used to evaluate the quality of the data for the groundwater samples. Blanks rarely contained detectable concentrations of most constituents, suggesting that contamination from sample collection procedures was not a significant source of bias in the data for the groundwater samples. Replicate samples generally were within the limits of acceptable analytical reproducibility. Median matrixspike recoveries were within the acceptable range (70 to 130 percent) for approximately 92 percent of the organic compounds.

All constituents were analyzed for in samples from the 112 wells in the Hard Rock study unit, except for chromium species, which were analyzed for the San Gabriel study area only. Of the 149 organic and special-interest constituents analyzed, 34 were detected in groundwater samples; concentrations of all detected constituents were less than regulatory and nonregulatory health-based benchmarks. One or more organic constituents were detected in 51 percent of the grid wells in the Hard Rock study unit: VOCs were detected in 44 percent and pesticides and pesticide degradates were detected in 18 percent. Perchlorate was detected in 48 percent of the Hard Rock grid wells.

Most of the grid-well samples analyzed for inorganic constituents had concentrations less than the regulatory and the nonregulatory health-based benchmarks. Arsenic was detected at concentrations greater than the USEPA maximum contaminant level (MCL-US) in three grid-well samples. Boron was detected at concentrations greater than the $\mathrm{CDPH}$ notification level (NL-CA) in three grid-well samples. Molybdenum was detected at a concentration greater than the USEPA lifetime health advisory level (HAL-US) in two gridwell samples. Nitrite plus nitrate (as nitrogen) was detected at concentrations greater than the CDPH maximum contaminant level (MCL-CA) in two grid-well samples. Fluoride was detected at concentrations greater than the MCL-CA in three grid-well samples.

Most of the grid-well samples analyzed for inorganic constituents had concentrations less than the non-enforceable benchmarks set for aesthetic concerns (secondary maximum contaminant levels [SMCLs]). Iron was detected at concentrations greater than the CDPH SMCL (SMCL-CA) in 19 grid-well samples, and manganese was detected at concentrations greater than the SMCL-CA in 27 grid-well samples. Chloride was detected at a concentration greater than the recommended SMCL-CA in three grid-well samples, one of which was also greater than the upper benchmark. Sulfate was detected at concentrations greater than the recommended SMCL-CA in four grid-well samples, none of which were greater than the upper benchmark. Total dissolved solids (TDS) concentrations were greater than the recommended SMCL-CA in 27 grid-well samples, 3 of which were also greater than the upper benchmark.

Radon-222 was detected at activities greater than the proposed MCL-US in five grid-well samples. Unadjusted gross alpha radioactivity was detected at activities greater than the MCL-US in 11 grid-well samples, 7 of which also had total uranium concentrations greater than the MCL-US and (or) uranium activities greater than the MCL-CA.

For the understanding-well samples, two detections of arsenic and one detection of fluoride had concentrations greater than the respective MCL-CA, one detection of strontium had a concentration greater than the HAL-US, and four detections of nitrite plus nitrate (as nitrogen) had concentrations greater than the MCL-US. Two detections of iron and eight detections of manganese were at concentrations greater than the respective SMCL-CA. Four detections of chloride were at concentrations greater than the recommended SMCL-CA, two of which were also greater than the upper benchmark. Two detections of sulfate were at concentrations greater than the recommended SMCL-CA. Nine detections of TDS were at concentrations greater than the recommended SMCL-CA, three of which were greater than the upper benchmark. 
Five understanding-well samples had radon-222 activities greater than the proposed MCL-US. One understandingwell sample had gross beta radioactivity greater than the MCL-CA. Seven understanding-well samples had gross alpha radioactivity greater than the MCL-US, five of which also had total uranium concentrations greater than the MCL-US and uranium activities greater than the MCL-CA. One understanding-well sample had uranium concentration and activity greater than the benchmarks, but gross alpha and beta activities were not analyzed for this sample.

\section{Acknowledgments}

The authors thank the following cooperators for their support: the California State Water Resources Control Board, California Department of Public Health, California Department of Water Resources, and Lawrence Livermore National Laboratory. We especially thank the well owners and water purveyors for allowing the U.S. Geological Survey to collect samples from their wells. Funding for this work was provided by State of California bonds authorized by Proposition 50 and administered by the State Water Resources Control Board.

\section{References}

American Public Health Association, 1998, Standard methods for the examination of water and wastewater (20th ed.): Washington, D.C., American Public Health Association, American Water Works Association, and Water Environment Federation, p. 3-37-3-43.

American Society for Testing and Materials, 1998, Water and environmental technology, in Annual Book of ASTM Standards: Philadelphia, Pa., American Society for Testing and Materials, section 11.02 (Water II), p. 664-666.

American Society for Testing and Materials, 2009, Standard test method for isotopic uranium in water by radiochemistry: American Society for Testing and Materials International, D3972-09, 7 p.

Anderson, C.W., 2005, Turbidity (ver. 2.1): U.S. Geological Survey Techniques of Water-Resources Investigations, book 9, chap. A6.7, accessed April 2, 2013, at http://water. usgs.gov/owq/FieldManual/Chapter6/6.7 contents.html.

Anderson, R.L., 1987, Practical statistics for analytical chemists: New York, Van Nostrand Reinhold Company, Inc., $315 \mathrm{p}$.

Ball, J.W., and McCleskey, R.B., 2003a, A new cationexchange method for accurate field speciation of hexavalent chromium: U.S. Geological Survey Water-Resources Investigations Report 2003-4018, 17 p. (Also available at http://pubs.er.usgs.gov/publication/wri034018.)
Ball, J.W., and McCleskey, R.B., 2003b, A new cationexchange method for accurate field speciation of hexavalent chromium: Talanta, v. 61, no. 3, p. 305-313, accessed October 20, 2014, at http://dx.doi.org/10.1016/S00399140(03)00282-0.

Belitz, Kenneth, Dubrovsky, N.M., Burow, Karen, Jurgens, Bryant, and Johnson, Tyler, 2003, Framework for a ground-water quality monitoring and assessment program for California: U.S. Geological Survey Water-Resources Investigations Report 2003-4166, 78 p. (Also available at http://pubs.er.usgs.gov/publication/wri034166.)

Bullen, T.D., Krabbenhoft, D.P., and Kendall, Carol, 1996, Kinetic and mineralogic controls on the evolution of groundwater chemistry and ${ }^{87} \mathrm{Sr} /{ }^{86} \mathrm{Sr}$ in a sandy silicate aquifer, northern Wisconsin, USA: Geochimica et Cosmochimica Acta, v. 60, no. 10, p. 1807-1821, accessed October 20, 2014, at http://dx.doi.org/10.1016/00167037(96)00052-X.

California Department of Public Health, 2012a, California drinking water-related laws-Drinking water-related regulations, Title 22: California Department of Public Health, accessed January 17, 2013, at http://www.waterboards.ca.gov/drinking_water/certlic/ drinkingwater/Lawbook.shtml.

California Department of Public Health, 2012b, Drinking water notification levels-Notification levels: California Department of Public Health, accessed January 17, 2013, at http://www.cdph.ca.gov/certlic/drinkingwater/Pages/ NotificationLevels.aspx.

California Department of Public Health, 2012c, Perchlorate in drinking water: California Department of Public Health, accessed January 17, 2013, at http://www.waterboards. ca.gov/drinking_water/certlic/drinkingwater/Perchlorate. $\underline{\text { shtml. }}$

California Department of Public Health, 2012d, Nitrates and nitrites in drinking water: California Department of Public Health, accessed January 17, 2013, at http://www.cdph. ca.gov/certlic/drinkingwater/Pages/Nitrate.aspx.

California Department of Water Resources, 2003, California's groundwater update 2003: California Department of Water Resources Bulletin 118, 246 p., accessed January 18, 2013, at http://www.water.ca.gov/groundwater/bulletin118/ update2003.cfm.

California Department of Water Resources, 2004a, California's groundwater-Individual basin descriptions, Upper Santa Ana Valley Groundwater Basin, Cajon Subbasin: California Department of Water Resources Bulletin 118, accessed January 18, 2013, at http://www.water.ca.gov/groundwater/ bulletin118.cfm. 
California Department of Water Resources, 2004b, California's groundwater-Individual basin descriptions, Hungry Valley Groundwater Basin: California Department of Water Resources Bulletin 118, accessed January 18, 2013, at http://www.water.ca.gov/pubs/groundwater/ bulletin 118/basindescriptions/4-18.pdf.

California Department of Water Resources, 2004c, California's groundwater-Individual basin descriptions, San Gabriel Valley Groundwater Basin: California Department of Water Resources Bulletin 118, accessed January 18, 2013, at http://www.water.ca.gov/pubs/groundwater/bulletin_118/ basindescriptions/4-13.pdf.

California Department of Water Resources, 2011, Water Facts 1 - Ground water in fractured hard rock, accessed January 18, 2013, at http://www.water.ca.gov/ waterconditions/drought/docs/water_facts_1.pdf.

California State Water Resources Control Board, 2003, Report to the Governor and Legislature, A comprehensive groundwater quality monitoring program for California: Assembly Bill 599, March 2003, 121 p., accessed January 18, 2013, at http://www.waterboards.ca.gov/gama/ docs/final_ab_599_rpt to legis 7 31_03.pdf.

California State Water Resources Control Board, 2009, GeoTracker GAMA: State Water Resources Control Board database, accessed January 18, 2013, at http://geotracker.waterboards.ca.gov/gama/.

California State Water Resources Control Board, 2011, GAMA - Groundwater Ambient Monitoring and Assessment Program: State Water Resources Control Board, accessed January 18, 2013, at http://www.waterboards. ca.gov/water issues/programs/gama/.

Childress, C.J.O., Foreman, W.T., Connor, B.F., and Maloney, T.J., 1999, New reporting procedures based on long-term method detection levels and some considerations for interpretations of water-quality data provided by the U.S. Geological Survey National Water Quality Laboratory: U.S. Geological Survey Open-File Report 99-193, 19 p. (Also available at http://pubs.er.usgs.gov/publication/ ofr99193.)

Clark, I.D., and Fritz, P., 1997, Environmental isotopes in hydrogeology: Boca Raton, Fla., CRC Press LLC, 328 p.

Connor, B.F., Rose, D.L., Noriega, M.C., Murtagh, L.K., and Abney, S.R., 1998, Methods of analysis by the U.S. Geological Survey National Water Quality Laboratory-Determination of 86 volatile organic compounds in water by gas chromatography/mass spectrometry, including detections less than reporting limits: U.S. Geological Survey Open-File Report 97-829, $78 \mathrm{p}$. (Also available at http://pubs.er.usgs.gov/publication/ ofr97829.)
Coplen, T.B., 1994, Reporting of stable hydrogen, carbon, and oxygen isotopic abundances: Pure and Applied Chemistry, v. 66, no. 2, p. 273-276, accessed October 20, 2014, at http://dx.doi.org/10.1351/pac199466020273.

Coplen, T.B., 2011, Guidelines and recommended terms for expression of stable-isotope-ratio and gas-ratio measurement results: Rapid Communication in Mass Spectrometry, v. 25 , no. 17 , p. $2538-2560$, accessed October 20, 2014, at http://onlinelibrary.wiley.com/doi/10.1002/ rcm.5129/abstract.

Coplen, T.B., Hopple, J.A., Böhlke, J.K., Peiser, H.S., Rieder, S.E., Krouse, H.R., Rosman, K.J.R., Ding, T., Vocke, R.D., Jr., Révész, K.M., Lamberty, A., Taylor, P., and DeBièvre, P., 2002, Compilation of minimum and maximum isotope ratios of selected elements in naturally occurring terrestrial materials and reagents: U.S. Geological Survey Water-Resources Investigations Report 2001-4222, 98 p. (Also available at http://pubs.er.usgs.gov/publication/ wri014222.)

Coplen, T.B., Wildman, J.D., and Chen, Julie, 1991, Improvements in the gaseous hydrogen-water equilibrium technique for hydrogen isotope-ratio analysis: Analytical Chemistry, v. 63, no. 9, p. 910-912, accessed October 20, 2014, at http://dx.doi.org/10.1021/ ac00009a014.

Davis, T.A., Olsen, L.D., Fram, M.S., and Belitz, Kenneth, 2014, Updated study reporting levels (SRLs) for traceelement data collected for the California Groundwater Ambient Monitoring and Assessment (GAMA) Priority Basin Project, October 2009-March 2013: U.S. Geological Survey Scientific Investigations Report 2014-5105, 52 p. (Also available at http://pubs.usgs.gov/sir/2014/5105/.)

Donahue, D.J., Linick, T.W., and Jull, A.J.T., 1990, Isotoperatio and background corrections for accelerator mass spectrometry radiocarbon measurements: Radiocarbon, v. 32, book 2, p. 135-142.

Dwyer, G.S., and Vengosh, A., 2008, Alternative filament loading solution for accurate analysis of boron isotopes by negative thermal ionization mass spectrometry: American Geophysical Union, Fall Meeting 2008, abstract \#H51C-0824.

Eaton, G.F., Hudson, G.B., and Moran, J.E., 2004, Tritiumhelium-3 age-dating of groundwater in the Livermore Valley of California: American Chemical Society (ACS) Symposium Series, v. 868, chap. 16, p. 235-245, accessed October 20, 2014, at http://dx.doi.org/10.1021/bk-2004-0868. ch016. 
Epstein, Samuel, and Mayeda, T.K., 1953, Variation of $\mathrm{O}^{18}$ content of waters from natural sources: Geochimica et Cosmochimica Acta, v. 4, no. 5, p. 213-224, accessed October 20, 2014, at http://dx.doi.org/10.1016/00167037(53)90051-9.

Faires, L.M., 1993, Methods of analysis by the U.S. Geological Survey National Water Quality Laboratory-Determination of metals in water by inductively coupled plasma-mass spectrometry: U.S. Geological Survey Open-File Report 92-634, 28 p. (Also available at http://pubs.er.usgs.gov/publication/ ofr92634.)

Farrar, J.W., and Long, H.K., 1997, Report on the U.S. Geological Survey's evaluation program for standard reference samples distributed in September 1996-T-143 (trace constituents), T-145 (trace constituents), M-140 (major constituents), N-51 (nutrient constituents), P-27 (low ionic strength constituents), and Hg-23 (mercury): U.S. Geological Survey Open-File Report 97-20, 145 p. (Also available at http://pubs.er.usgs.gov/publication/ ofr9720.)

Faure, Gunter, and Mensing, T.M., 2005, Isotopes-Principles and applications (3d ed.): Hoboken, New Jersey, John Wiley \& Sons, Inc., $928 \mathrm{p}$.

Ferrari, M.J., Fram, M.S., and Belitz, Kenneth, 2008, Ground-water quality in the central Sierra study unit, 2006-Results from the California GAMA Program: U.S. Geological Survey Data Series 335, 61 p. (Also available at http://pubs.er.usgs.gov/publication/ds335.)

Fishman, M.J., ed., 1993, Methods of analysis by the U.S. Geological Survey National Water Quality Laboratory-Determination of inorganic and organic constituents in water and fluvial sediments: U.S. Geological Survey Open-File Report 93-125, 217 p. (Also available at http://pubs.er.usgs.gov/publication/ ofr93125.)

Fishman, M.J., and Friedman, L.C., eds., 1989, Methods for determination of inorganic substances in water and fluvial sediments: U.S. Geological Survey Techniques of WaterResources Investigations, book 5, chap. A1, 545 p. (Also available at http://pubs.er.usgs.gov/publication/twri05A1.)

Fram, M.S., and Belitz, Kenneth, 2007, Groundwater quality data in the southern Sierra study unit, 2006-Results from the California GAMA Program: U.S. Geological Survey Data Series 301, 78 p. (Also available at http://pubs.er.usgs.gov/publication/ds301.)

Fram, M.S., Munday, Cathy, and Belitz, Kenneth, 2009, Groundwater quality data for the Tahoe-Martis study unit, 2007-Results from the California GAMA Program: U.S. Geological Survey Data Series 432, 88 p. (Also available at http://pubs.usgs.gov/ds/432/.)
Fram, M.S., Olsen, L.D., and Belitz, Kenneth, 2012, Evaluation of volatile organic compound (VOC) blank data and application of study reporting levels to groundwater data collected for the California GAMA Priority Basin Project, May 2004 through September 2010: U.S. Geological Survey Scientific Investigations Report 2012-5139, 94 p. (Also available at http://pubs.usgs.gov/sir/2012/5139/.)

Gagnon, A.R., and Jones, G.A., 1993, AMS-graphite target production methods at the Woods-Hole Oceanographic Institution during 1986-1991: Radiocarbon, v. 35, book 2, p. $301-310$.

Garbarino, J.R., 1999, Methods of analysis by the U.S. Geological Survey National Water Quality LaboratoryDetermination of dissolved arsenic, boron, lithium, selenium, strontium, thallium, and vanadium using inductively coupled plasma-mass spectrometry: U.S. Geological Survey OpenFile Report 99-93, 31 p. (Also available at http://pubs. er.usgs.gov/publication/ofr9993.)

Garbarino, J.R., Kanagy, L.K., and Cree, M.E., 2006, Determination of elements in natural-water, biota, sediment, and soil samples using collision/reaction cell inductively coupled plasma-mass spectrometry: U.S. Geological Survey Techniques and Methods, book 5, chap. B1, 87 p. (Also available at $\mathrm{http}: / /$ pubs.er.usgs.gov/publication/tm5B1.)

Gilliom, R.J., Barbash, J.E., Crawford, C.G., Hamilton, P.A., Martin, J.D., Nakagaki, Naomi, Nowell, L.H., Scott, J.C., Stackelberg, P.E., Thelin, G.P., and Wolock, D.M., 2006, The quality of our Nation's waters-Pesticides in the Nation's streams and ground water, 1992-2001: U.S. Geological Survey Circular 1291, 173 p. (Also available at http://pubs.usgs.gov/circ/2005/1291/.)

Goldrath, D.A., Wright, M.T., and Belitz, Kenneth, 2009, Ground-water quality data in the Coachella Valley study unit, 2007-Results from the California GAMA Program: U.S. Geological Survey Data Series 373, 71 p. (Also available at http://pubs.usgs.gov/ds/373/.)

Gran, Gunnar, 1952, Determination of the equivalence point in potentiometric titration, Part II: Analyst, v. 77, p. 661-671.

Hahn, G.J., and Meeker, W.Q., 1991, Statistical intervals-A guide for practitioners: New York, John Wiley \& Sons Inc., 397 p., accessed October 20, 2014, at http://dx.doi. org/10.1002/9780470316771.

Hem, J.D., 1985, Study and interpretation of the chemical characteristics of natural water: U.S. Geological Survey Water-Supply Paper 2254, 264 p. (Also available at http://pubs.usgs.gov/wsp/wsp2254/.) 
Hoaglin, D.C., 1983, Letter values-A set of selected order statistics, in Hoaglin, D.C., Mosteller, Frederick, and Tukey, J.W., eds., Understanding robust and exploratory data analysis: New York, John Wiley \& Sons, p. 33-54.

Kenny, J.F., Barber, N.L., Hutson, S.S., Linsey, K.S., Lovelace, J.K., and Maupin, M.A., 2009, Estimated use of water in the United States in 2005: U.S. Geological Survey Circular 1344, 60 p. (Also available at http:/pubs.usgs.gov/circ/1344/.)

Kent, Robert, and Belitz, Kenneth, 2009, Ground-water quality data in the upper Santa Ana Watershed study unit, November 2006 to March 2007-Results from the California GAMA Program: U.S. Geological Survey Data Series 404, 116 p. (Also available at http:// pubs.usgs.gov/ds/404/.)

Koterba, M.T., Wilde, F.D., and Lapham, W.W., 1995, Ground-water data-collection protocols and procedures for the National Water-Quality Assessment Program - Collection and documentation of water-quality samples and related data: U.S. Geological Survey OpenFile Report 95-399, 114 p. (Also available at http://pubs. er.usgs.gov/publication/ofr95399.)

Krieger, H.L., and Whittaker, E.L., 1980, Prescribed procedures for measurement of radioactivity in drinking water: U.S. Environmental Protection Agency EPA-600/4-80-032, 142 p. (Also available as PB80-224744 at http://www.ntis.gov/search/product. aspx?abbr=PB80224744.)

Kulongoski, Justin, and Belitz, Kenneth, 2004, Groundwater ambient monitoring and assessment program: U.S. Geological Survey Fact Sheet 2004-3088, 2 p. (Also available at http://pubs.usgs.gov/fs/2004/3088/.)

Kulongoski, J.T., and Belitz, Kenneth, 2007, Ground-water quality data in the Monterey Bay and Salinas Valley basins, California, 2005-Results from the California GAMA Program: U.S. Geological Survey Data Series 258, 84 p. (Also available at http://pubs.usgs.gov/ds/2007/258/.)

Kulongoski, J.T., Belitz, Kenneth, and Dawson, B.J., 2006, Ground-water quality data in the north San Francisco Bay hydrologic provinces, California, 2004-Results from the California GAMA Program: U.S. Geological Survey Data Series 167, 100 p. (Also available at http://pubs.usgs.gov/ds/ds167/.)

Land, Michael, and Belitz, Kenneth, 2008, Ground-water quality data in the San Fernando-San Gabriel study unit, 2005-Results from the California GAMA Program: U.S. Geological Survey Data Series 356, 84 p. (Also available at http://pubs.usgs.gov/ds/356/.)
Landon, M.K., Belitz, Kenneth, Jurgens, B.C., Kulongoski, J.T., and Johnson, T.D., 2010, Status and understanding of groundwater quality in the CentralEastside San Joaquin Basin, 2006 - California GAMA Priority Basin Project: U.S. Geological Survey Scientific Investigations Report 2009-5266, 97 p. (Also available at http:/pubs.usgs.gov/sir/2009/5266/.)

Lane, S.L., Flanagan, Sarah, and Wilde, F.D., 2003, Selection of equipment for water sampling (ver. 2.0): U.S. Geological Survey Techniques of Water-Resources Investigations, book 9, chap. A2, accessed January 18, 2013, at http://water. usgs.gov/owq/FieldManual/Chapter2/Ch2 contents.html.

Lewis, M.E., 2006, Dissolved oxygen (ver. 2.1):

U.S. Geological Survey Techniques of Water-Resources Investigations, book 9, chap. A6.2, accessed January 18, 2013, at http://water.usgs.gov/owq/FieldManual/ Chapter6/6.2 contents.html.

Lindley, C.E., Stewart, J.T., and Sandstrom, M.W., 1996, Determination of low concentrations of acetochlor in water by automated solid-phase extraction and gas chromatography with mass-selective detection: Journal of AOAC International, v. 79, no. 4, p. 962-966, accessed October 20, 2014, at http://nwql.usgs.gov/pubs/JOUR/ Acetochlor.pdf.

Madsen, J.E., Sandstrom, M.W., and Zaugg, S.D., 2002, Methods of analysis by the U.S. Geological Survey National Water Quality Laboratory-A method supplement for the determination of Fipronil and degradates in water by gas chromatography/mass spectrometry: U.S. Geological Survey Open-File Report 2002-462, 11 p. (Also available at http://pubs.er.usgs.gov/publication/ofr02462.)

Mathany, T.M., and Belitz, Kenneth, 2013, Groundwaterquality data in the Bear Valley and selected Hard Rock Areas study unit, 2010-Results from the California GAMA Program: U.S. Geological Survey Data Series 747, 86 p. (Also available at http://pubs.usgs.gov/ds/747/.)

Mathany, T.M., and Belitz, Kenneth, 2014, Groundwaterquality data in the Klamath Mountains study unit, 2010 - Results from the California GAMA Program: U.S. Geological Survey Data Series 803, 82 p. (Also available at http://pubs.usgs.gov/ds/803/.)

Mathany, T.M., Land, Michael, and Belitz, Kenneth, 2008, Ground-water quality data in the Coastal Los Angeles Basin study unit, 2006 - Results from the California GAMA Program: U.S. Geological Survey Data Series 387, 98 p. (Also available at http://pubs.usgs.gov/ds/387/.) 
McCleskey, R.B., Nordstrom, D.K., and Ball, J.W., 2003, Metal interferences and their removal prior to the determination of $\mathrm{As}(\mathrm{T})$ and $\mathrm{As}(\mathrm{III})$ in acid mine waters by hydride generation atomic absorption spectrometry: U.S. Geological Survey Water-Resources Investigations Report 2003-4117, 20 p. (Also available at http://pubs.usgs.gov/wri/wri03-4117/.)

McCurdy, D.E., Garbarino, J.R., and Mullin, A.H., 2008, Interpreting and reporting radiological water-quality data: U.S. Geological Survey Techniques and Methods, book 5, chap. B6, 33 p. (Also available at http://pubs.usgs.gov/ $\underline{\mathrm{tm} / 05 \mathrm{~b} 06 / .)}$

McLain, B.J., 1993, Methods of analysis by the U.S. Geological Survey National Water Quality Laboratory-Determination of chromium in water by graphite furnace atomic absorption spectrophotometry: U.S. Geological Survey Open-File Report 93-449, 16 p. (Also available at http://pubs.er.usgs.gov/publication/ ofr93449.)

McNichol, A.P., Gagnon, A.R., Jones, G.A., and Osborne, E.A., 1992, Illumination of a black box-Analysis of gas composition during graphite target preparation, in Long, A., and Kra, R.S., eds., Proceedings of the 14th International ${ }^{14} \mathrm{C}$ Conference: Radiocarbon, v. 34, no. 3, p. 321-329.

McNichol, A.P., Jones, G.A., Hutton, D.L., and Gagnon, A.R., 1994, The rapid preparation of seawater $\sum \mathrm{CO}_{2}$ for radiocarbon analysis at the National Ocean Sciences AMS Facility: Radiocarbon, v. 36, no. 2, p. 237-246.

Montrella, Joseph, and Belitz, Kenneth, 2009, Ground-water quality data in the Santa Clara River Valley study unit, 2007-Results from the California GAMA Program: U.S. Geological Survey Data Series 408, 84 p. (Also available at http://pubs.usgs.gov/ds/408/.)

Moran, J.E., Hudson, G.B., Eaton, G.F., and Leif, Roald, 2002, A contamination vulnerability assessment for the Livermore-Amador and Niles Cone Groundwater Basins: Report to the California State Water Resources Board, UCRL-AR-148831, 25 p., accessed October 20, 2014, at http://www.waterboards.ca.gov/publications forms/ publications/general/docs/gama.pdf.

Mueller, D.K., and Titus, C.J., 2005, Quality of nutrient data from streams and ground water sampled during water years 1992-2001: U.S. Geological Survey Scientific Investigations Report 2005-5106, 27 p. (Also available at http://pubs.er.usgs.gov/publication/sir20055106.)
Olsen, L.D., Fram, M.S., and Belitz, Kenneth, 2010, Review of trace-element field-blank data collected for the California Groundwater Ambient Monitoring and Assessment (GAMA) Program, May 2004January 2008: U.S. Geological Survey Scientific Investigations Report 2009-5220, 47 p. (Also available at http://pubs.usgs.gov/sir/2009/5220/.)

Patton, C.J., and Kryskalla, J.R., 2003, Methods of analysis by the U.S. Geological Survey National Water Quality Laboratory-Evaluation of alkaline persulfate digestion as an alternative to Kjeldahl digestion for determination of total and dissolved nitrogen and phosphorous in water: U.S. Geological Survey WaterResources Investigations Report 2003-4174, 33 p. (Also

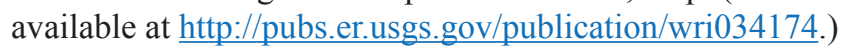

Patton, C.J., and Kryskalla, J.R., 2011, Colorimetric determination of nitrate plus nitrite in water by enzymatic reduction, automated discrete analyzer methods: U.S. Geological Survey Techniques and Methods, book 5, chap. B8, 34 p. (Also available at http://pubs.er.usgs.gov/ publication/tm5B8.)

Radtke, D.B., Davis, J.V., and Wilde, F.D., 2005, Specific electrical conductance (ver. 1.2): U.S. Geological Survey Techniques of Water-Resources Investigations, book 9, chap. A6.3, accessed January 18, 2013, at http://water.usgs. gov/owq/FieldManual/Chapter6/6.3 contents.html.

Ray, M.C., Kulongoski, J.T., and Belitz, Kenneth, 2009, Ground-water quality data in the San Francisco Bay study unit, 2007-Results from the California GAMA Program: U.S. Geological Survey Data Series 396, 93 p. (Also available at http://pubs.usgs.gov/ds/396/.)

Ritz, G.F., and Collins, J.A., 2008, pH (ver. 2.0): U.S. Geological Survey Techniques of Water-Resources Investigations, book 9, chap. A6.4, accessed January 18, 2013, at http://water.usgs.gov/owq/FieldManual/ Chapter6/6.4 contents.html.

Sandstrom, M.W., Stroppel, M.E., Foreman, W.T., and Schroeder, M.P., 2001, Methods of analysis by the U.S. Geological Survey National Water Quality Laboratory-Determination of moderateuse pesticides and selected degradates in water by C-18 solid-phase extraction and gas chromatography/ mass spectrometry: U.S. Geological Survey WaterResources Investigations Report 2001-4098, 70 p. (Also available at http://pubs.er.usgs.gov/publication/ wri20014098.)

Saucedo, G.J., Bedford, D.R., Raines, G.L., Miller, R.J., and Wentworth, C.M., 2000, GIS data for the geologic map of California: California Geological Survey, CD 2000-07. 
Schmitt, S.J., Milby Dawson, B.J., and Belitz, Kenneth, 2009, Groundwater-quality data in the Antelope Valley study unit, 2008 - Results from the California GAMA Program: U.S. Geological Survey Data Series 479, 79 p. (Also available at http://pubs.usgs.gov/ds/479/.)

Schneider, R.J., Jones, G.A., McNichol, A.P., von Reden, K.F., Elder, K.A., Huang, K., and Kessel, E.D., 1994, Methods for data screening, flagging and error analysis at the National Ocean Sciences AMS Facility: Nuclear Instruments and Methods in Physics Research, book 92, p. $172-175$.

Scott, J.C., 1990, Computerized stratified random siteselection approaches for design of a ground-waterquality sampling network: U.S. Geological Survey Water-Resources Investigations Report 90-4101, 109 p. (Also available at http://pubs.er.usgs.gov/publication/ wri904101.)

Shelton, J.L., Burow, K.R., Belitz, Kenneth, Dubrovsky, N.M., Land, Michael, and Gronberg, JoAnn, 2001, Low-level volatile organic compounds in active public supply wells as ground-water tracers in the Los Angeles physiographic basin, California, 2000: U.S. Geological Survey WaterResources Investigations Report 2001-4188, 35 p. (Also available at http://pubs.er.usgs.gov/publication/ wri20014188.)

Shelton, J.L., Fram, M.S., Munday, C.M., and Belitz, Kenneth, 2010, Groundwater-quality data for the Sierra Nevada study unit, 2008-Results from the California GAMA Program: U.S. Geological Survey Data Series 534, 82 p. (Also available at http://pubs.usgs.gov/ds/534/.)

Soddy, F., 1913, Radioactivity-Annual Reports on the progress of chemistry, v. 10 , p. 262-288.

State of California, 2001a, Assembly Bill No. 599, Chapter 522, accessed January 18, 2013, at http://www.swrcb.ca.gov/ gama/docs/ab_599 bill 20011005 chaptered.pdf.

State of California, 2001b, Groundwater Monitoring Act of 2001: California Water Code, part 2.76, Sections 10780-10782.3, accessed January 18, 2013, at http://www.leginfo.ca.gov/.

Stevenson, D.L., ed., 2013, Quality management system, U.S. Geological Survey National Water Quality Laboratory: unpublished U.S. Geological Survey Report, version 2.1, 28 May 2013, 74 p., accessed January 6, 2014, at http://nwql.usgs.gov/qms v2.shtml.

Stookey, L.L., 1970, Ferrozine-A new spectrophotometric reagent for iron: Analytical Chemistry, v. 42, no. 7 , p. 779-781, accessed October 20, 2014, at http://dx.doi. org/10.1021/ac60289a016.
Stumm, Werner, and Morgan, J.J., 1996, Aquatic chemistryChemical equilibria and rates in natural waters ( $3 \mathrm{~d}$ ed.): New York, John Wiley and Sons, 1022 p.

Tanaka, H., Smith, T.E., and Huang, C.H., 1984, The Santiago Peak volcanic rocks of the Peninsular Ranges batholith, Southern California-Volcanic rocks associated with coeval gabbros: Bulletin Volcanologique, v. 47, no. 1, p. 153-171.

Thatcher, L.L., Janzer, V.J., and Edwards, K.W., 1977, Methods for the determination of radioactive substances in water and fluvial sediments: U.S. Geological Survey Techniques of WaterResources Investigations, book 5, chap. A5, 95 p. (Also available at http://pubs.er.usgs.gov/publication/twri05A5.)

Timme, P.J., 1995, National Water Quality Laboratory, 1995 services catalog: U.S. Geological Survey Open-File Report 95-352, 120 p. (Also available at http:// pubs.er.usgs.gov/publication/ofr95352.)

To, T.B., Nordstrom, D.K., Cunningham, K.M., Ball, J.W., and McCleskey, R.B., 1999, New method for the direct determination of dissolved Fe(III) concentration in acid mine waters: Environmental Science \& Technology, v. 33, no. 5, p. 807-813, accessed October 20, 2014, at http:// dx.doi.org/10.1021/es980684z.

U.S. Department of Commerce, National Climatic Data Center, 2011, National Oceanic \& Atmospheric Administration home page, accessed May 8, 2013, at http:// www.ncdc.noaa.gov/cdo-web/search?datasetid=ANNUAL.

U.S. Environmental Protection Agency, 2002, Guidelines for establishing procedures for the analysis of pollutants: U.S. Code of Federal Regulations, Title 40, 136 p.

U.S. Environmental Protection Agency, 2005, Method 331.0 Determination of perchlorate in drinking water by liquid chromatography electrospray ionization mass spectrometry (Revision 1.0, January 2005): Office of Groundwater and Drinking Water, U.S. Environmental Protection Agency Document \#815-R-05-007, 34 p., accessed January 18, 2013, at http://www.epa.gov/safewater/methods/pdfs/ methods/met331 0.pdf.

U.S. Environmental Protection Agency, 2009, Drinking water contaminants, accessed January 18, 2013, at http://www.epa.gov/safewater/contaminants/index.html.

U.S. Environmental Protection Agency, 2012a, Drinking water health advisories - 2012 Drinking water standards and health advisory tables, accessed January 18, 2013, at http://www.epa.gov/waterscience/criteria/drinking/.

U.S. Environmental Protection Agency, 2012b, Proposed radon in drinking water regulation, accessed January 18, 2013, at http://water.epa.gov/lawsregs/rulesregs/sdwa/ radon/regulations.cfm. 
U.S. Environmental Protection Agency, 2013, Basic information about radionuclides in drinking water, accessed March 10, 2014, at http://water.epa.gov/drink/contaminants/ basicinformation/radionuclides.cfm.

U.S. Geological Survey, [variously dated], National field manual for the collection of water-quality data: U.S. Geological Survey Techniques of Water-Resources Investigations, book 9, chap. A1-A9, accessed January 18, 2013, at http://water.usgs.gov/owq/FieldManual/.

U.S. Geological Survey, 2006, National Elevation Dataset: U.S. Geological Survey database, accessed January 18, 2013, at http://ned.usgs.gov/.

U.S. Geological Survey, 2010, California Quaternary Faults: U.S. Geological Survey Geologic Hazards Science Center website, accessed April 22, 2013, at http://geohazards.usgs.gov/qfaults/ca/California.php.

U.S. Geological Survey, 2012, Branch of Quality Systems, USGS Inorganic Blind Sample Project (IBSP), NWQL control charts for fiscal year 2011-2012 analytes, accessed January 18, 2013, at http://bqs.usgs.gov/ibsp/FY11 12charts.shtml.

U.S. Geological Survey, 2013a, What is GAMA?: U.S. Geological Survey California Water Science Center website, accessed January 18, 2013, at http://ca.water.usgs.gov/projects/gama/.

U.S. Geological Survey, 2013b, Publications: U.S. Geological Survey California Water Science Center website, accessed January 18, 2013, at http://ca.water.usgs.gov/projects/gama/ includes/GAMA publications.html.

U.S. Geological Survey, 2013c, Branch of Quality Systems, USGS Inorganic Blind Sample Project (IBSP), data quality assessment summaries, accessed January 18, 2013, at http://bqs.usgs.gov/ibsp/qadata.shtml.

U.S. Geological Survey, 2014, USGS water data for the Nation, accessed February 10, 2014, at http://waterdata.usgs.gov/nwis.

Vengosh, Avner, Chivas, A.R., and McCulloch, M.T., 1989, Direct determination of boron and chlorine isotopes compositions in geological materials by negative thermal-ionization mass spectrometry: Chemical Geology, v. 79, no. 4, p. 333-343.

Vogel, J.S., Nelson, D.E., and Southon, J.R., 1987, ${ }^{14} \mathrm{C}$ background levels in an accelerator mass spectrometry system: Radiocarbon, v. 29, no. 3, p. 323-333.

Weiss, R.F., 1968, Piggyback sampler for dissolved gas studies on sealed water samples: Deep Sea Research, v. 15, no. 6, p. 695-699, accessed October 20, 2014, at http://dx.doi.org/10.1016/0011-7471(68)90082-x.
Wilde, F.D., ed., 2004, Cleaning of equipment for water sampling (ver. 2.0): U.S. Geological Survey Techniques of Water-Resources Investigations, book 9, chap. A3, accessed January 18, 2013, at http://water.usgs.gov/owq/ FieldManual/chapter3/Ch3 contents.html.

Wilde, F.D., ed., 2006a, Collection of water samples: U.S. Geological Survey Techniques of Water-Resources Investigations, book 9, chap. A4, accessed January 18, 2013, at http://water.usgs.gov/owq/FieldManual/chapter4/ html/Ch4 contents.html.

Wilde, F.D., ed., 2006b, Temperature (ver. 2): U.S. Geological Survey Techniques of Water-Resources Investigations, book 9, chap. A6.1, accessed January 18, 2013, at http://water. usgs.gov/owq/FieldManual/Chapter6/6.1 contents.html.

Wilde, F.D., ed., 2008, Guidelines for field-measured waterquality properties (ver. 2.0): U.S. Geological Survey Techniques of Water-Resources Investigations, book 9, chap. A6.0, accessed January 18, 2013, at http://water.usgs. gov/owq/FieldManual/Chapter6/6.0 contents.html.

Wilde, F.D., ed., 2009, Processing of water samples (ver. 2.2): U.S. Geological Survey Techniques of Water-Resources Investigations, book 9, chap. A5, accessed January 18, 2013, at http://water.usgs.gov/owq/FieldManual/chapter5/ html/Ch5 contents.html.

Worthington, C.E., Sebastian, J.E., Gilmore, D.F., and Benfield, R.C., 2007, Investigative report of carbon disulfide contamination in powder-free latex exam gloves, in Tennessee Department of Environment and Conservation, Department of Energy Oversight Division, Environmental Monitoring Report, January through December 2006, p. 233-236.

Wright, M.T., Belitz, Kenneth, and Burton, C.A., 2005, California GAMA Program - Ground-water quality data in the San Diego drainages hydrogeologic province, California, 2004: U.S. Geological Survey Data Series 129, $91 \mathrm{p}$. (Also available at $\mathrm{http}: / /$ pubs.usgs.gov/ds/2005/129/.)

Zaugg, S.D., Sandstrom, M.W., Smith, S.G., and Fehlberg, K.M., 1995, Methods of analysis by the U.S. Geological Survey National Water Quality Laboratory-Determination of pesticides in water by $\mathrm{C}-18$ solid-phase extraction and capillarycolumn gas chromatography/mass spectrometry with selected-ion monitoring: U.S. Geological Survey Open-File Report 95-181, 60 p. (Also available at http:// pubs.er.usgs.gov/publication/ofr95181.)

Zogorski, J.S., Carter, J.M., Ivahnenko, Tamara, Lapham, W.W., Moran, M.J., Rowe, B.L., Squillace, P.J., and Toccalino, P.L., 2006, Volatile organic compounds in the Nation's ground water and drinking-water supply wells: U.S. Geological Survey Circular 1292, 101 p. (Also available at http://pubs.usgs.gov/circ/circ1292/.) 
Table 1. Identification, sampling, and construction information for wells sampled for the Santa Cruz, San Gabriel, and Peninsular Ranges Hard Rock Aquifers (Hard Rock) study unit, March 2011 through March 2012, California GAMA Priority Basin Project.

[GAMA well identification number: HR-SC, Santa Cruz study area grid well; HR-SG, San Gabriel study area grid well; HR-SGU, San Gabriel study area understanding well; HR-PR, Peninsular Ranges study area grid well; HR-PRFP, Peninsular Ranges study area flow-path well; HR-PRU, Peninsular Ranges study area understanding well. Other abbreviations: ft, feet; LSD, land-surface datum; NAVD 88, North American Vertical Datum of 1988; na, not available]

\begin{tabular}{|c|c|c|c|c|c|c|}
\hline $\begin{array}{c}\text { GAMA well } \\
\text { identification } \\
\text { number }\end{array}$ & $\begin{array}{l}\text { Date sampled } \\
\text { (mm/dd/yyyy) }\end{array}$ & $\begin{array}{c}\text { Altitude of LSD } \\
\text { (ft above NAVD 88) }\end{array}$ & Well type & $\begin{array}{c}\text { Well depth } \\
\text { (ft below LSD) }\end{array}$ & $\begin{array}{c}\text { Top of perforated } \\
\text { interval } \\
\text { (ft below LSD) }\end{array}$ & $\begin{array}{c}\text { Bottom of } \\
\text { perforated interval } \\
\text { (ft below LSD) }\end{array}$ \\
\hline \multicolumn{7}{|c|}{ Santa Cruz study area (25 wells) } \\
\hline HR-SC-01 & $3 / 28 / 2011$ & 482 & Production & 776 & 370 & 776 \\
\hline HR-SC-02 & $3 / 29 / 2011$ & 483 & Spring & na & na & na \\
\hline HR-SC-03 & $3 / 29 / 2011$ & 361 & Production & 140 & 80 & 140 \\
\hline HR-SC-05 & $3 / 31 / 2011$ & 2,450 & Production & 310 & 50 & 310 \\
\hline HR-SC-06 & $4 / 4 / 2011$ & 2,082 & Production & 260 & na & na \\
\hline HR-SC-07 & $4 / 4 / 2011$ & 445 & Production & 300 & 90 & 280 \\
\hline HR-SC-11 & $4 / 6 / 2011$ & 1,679 & Production & 110 & 60 & 110 \\
\hline HR-SC-12 & 4/7/2011 & 826 & Production & 226 & 126 & 226 \\
\hline HR-SC-13 & $4 / 18 / 2011$ & 1,000 & Production & 160 & 50 & 160 \\
\hline HR-SC-14 & $4 / 19 / 2011$ & 1,779 & Production & 180 & 160 & 180 \\
\hline HR-SC-15 & $4 / 19 / 2011$ & 622 & Production & 320 & 220 & 320 \\
\hline HR-SC-16 & $4 / 20 / 2011$ & 1,142 & Production & 293 & 60 & 280 \\
\hline HR-SC-17 & $4 / 20 / 2011$ & 1,013 & Production & 320 & na & na \\
\hline HR-SC-23 & $5 / 3 / 2011$ & 1,683 & Production & 480 & 90 & 480 \\
\hline HR-SC-24 & $5 / 4 / 2011$ & 134 & Production & 90 & 70 & 90 \\
\hline HR-SC-25 & $5 / 5 / 2011$ & 525 & Production & 174 & 124 & 164 \\
\hline \multicolumn{7}{|c|}{ San Gabriel study area (37 wells) } \\
\hline HR-SG-01 & $5 / 16 / 2011$ & 1,829 & Production & na & na & na \\
\hline HR-SG-02 & $5 / 16 / 2011$ & 2,717 & Production & na & 22 & 62 \\
\hline HR-SG-03 & $5 / 17 / 2011$ & 2,154 & Production & na & na & na \\
\hline HR-SG-04 & $5 / 18 / 2011$ & 2,364 & Production & 90 & na & na \\
\hline HR-SG-05 & $5 / 19 / 2011$ & 3,319 & Production & 805 & 238 & 805 \\
\hline
\end{tabular}


Table 1. Identification, sampling, and construction information for wells sampled for the Santa Cruz, San Gabriel, and Peninsular Ranges Hard Rock Aquifers (Hard Rock) study unit, March 2011 through March 2012, California GAMA Priority Basin Project.Continued

[GAMA well identification number: HR-SC, Santa Cruz study area grid well; HR-SG, San Gabriel study area grid well; HR-SGU, San Gabriel study area understanding well; HR-PR, Peninsular Ranges study area grid well; HR-PRFP, Peninsular Ranges study area flow-path well; HR-PRU, Peninsular Ranges study area understanding well. Other abbreviations: ft, feet; LSD, land-surface datum; NAVD 88, North American Vertical Datum of 1988; na, not available]

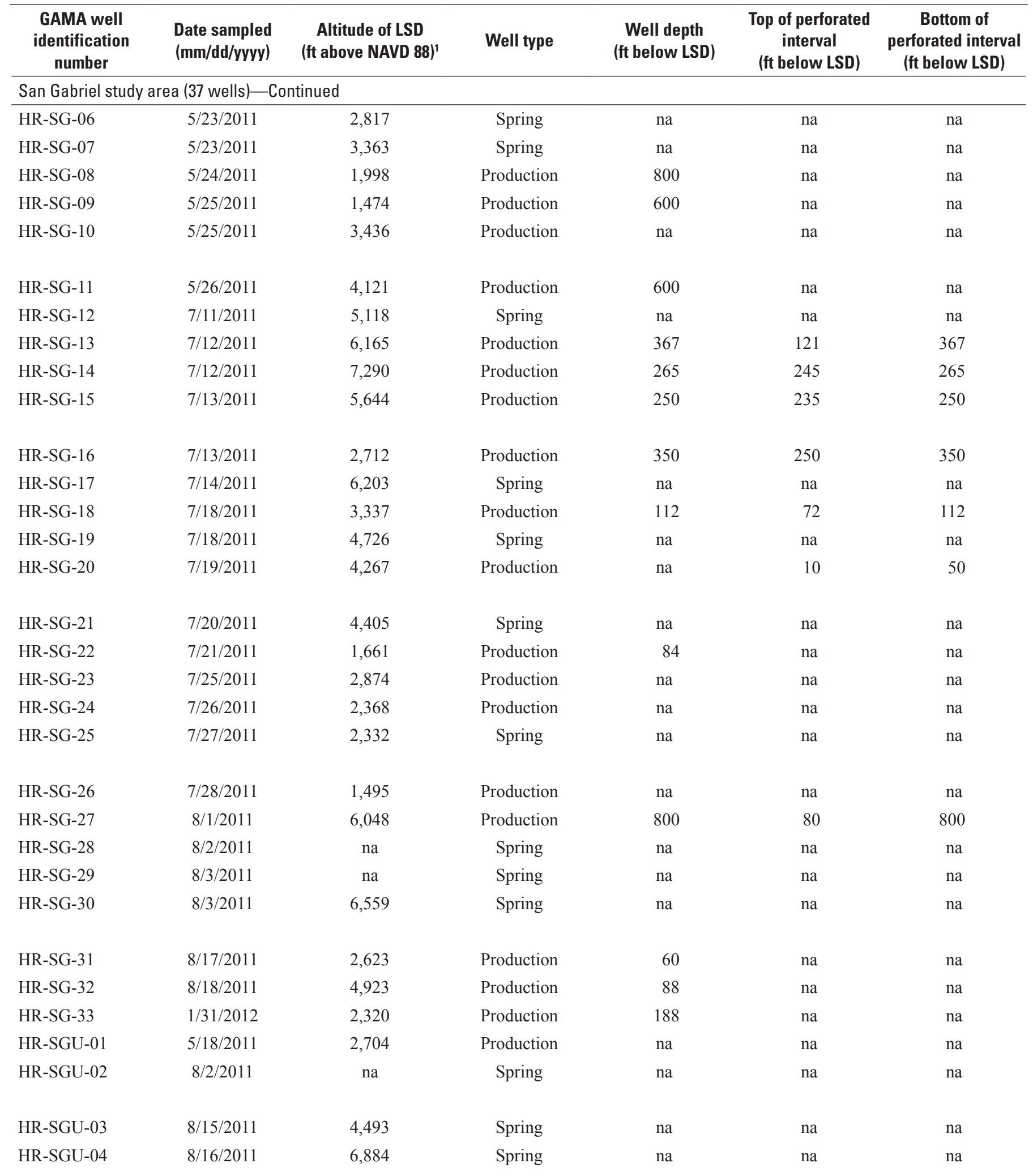


Table 1. Identification, sampling, and construction information for wells sampled for the Santa Cruz, San Gabriel, and Peninsular Ranges Hard Rock Aquifers (Hard Rock) study unit, March 2011 through March 2012, California GAMA Priority Basin Project.Continued

[GAMA well identification number: HR-SC, Santa Cruz study area grid well; HR-SG, San Gabriel study area grid well; HR-SGU, San Gabriel study area understanding well; HR-PR, Peninsular Ranges study area grid well; HR-PRFP, Peninsular Ranges study area flow-path well; HR-PRU, Peninsular Ranges study area understanding well. Other abbreviations: ft, feet; LSD, land-surface datum; NAVD 88, North American Vertical Datum of 1988; na, not available]

\begin{tabular}{|c|c|c|c|c|c|c|}
\hline $\begin{array}{c}\text { GAMA well } \\
\text { identification } \\
\text { number }\end{array}$ & $\begin{array}{l}\text { Date sampled } \\
\text { (mm/dd/yyyy) }\end{array}$ & $\begin{array}{c}\text { Altitude of LSD } \\
\text { (ft above NAVD 88) }\end{array}$ & Well type & $\begin{array}{c}\text { Well depth } \\
\text { (ft below LSD) }\end{array}$ & $\begin{array}{c}\text { Top of perforated } \\
\text { interval } \\
\text { (ft below LSD) }\end{array}$ & $\begin{array}{c}\text { Bottom of perforated } \\
\text { interval } \\
\text { (ft below LSD) }\end{array}$ \\
\hline \multicolumn{7}{|c|}{ Peninsular Ranges study area ( 50 wells) } \\
\hline HR-PR-01 & $8 / 22 / 2011$ & 2,081 & Production & 46 & 41 & na \\
\hline HR-PR-02 & $8 / 23 / 2011$ & 3,884 & Production & 222 & na & na \\
\hline HR-PR-03 & $8 / 23 / 2011$ & 4,757 & Production & 315 & na & na \\
\hline HR-PR-04 & $8 / 24 / 2011$ & 2,857 & Production & 280 & 119 & 280 \\
\hline HR-PR-05 & $8 / 24 / 2011$ & 3,894 & Production & 400 & 97 & 400 \\
\hline HR-PR-06 & $8 / 25 / 2011$ & 3,121 & Production & 500 & 60 & 500 \\
\hline HR-PR-07 & $8 / 29 / 2011$ & 342 & Production & 450 & 60 & 345 \\
\hline HR-PR-08 & $8 / 30 / 2011$ & 2,282 & Production & 560 & 320 & 560 \\
\hline HR-PR-09 & $8 / 31 / 2011$ & 4,813 & Production & 600 & 76 & 600 \\
\hline HR-PR-10 & 9/13/2011 & 4,107 & Production & 500 & na & 500 \\
\hline HR-PR-11 & $9 / 13 / 2011$ & 1,542 & Production & 979 & 820 & 979 \\
\hline HR-PR-12 & $9 / 14 / 2011$ & 1,003 & Production & 93.5 & na & na \\
\hline HR-PR-13 & $9 / 14 / 2011$ & 1,751 & Production & 59.5 & na & na \\
\hline HR-PR-14 & $9 / 15 / 2011$ & 1,650 & Production & 350 & 50 & 350 \\
\hline HR-PR-15 & $9 / 20 / 2011$ & 4,937 & Production & na & na & na \\
\hline HR-PR-16 & $9 / 21 / 2011$ & 658 & Production & 500 & 0 & 500 \\
\hline HR-PR-17 & $9 / 21 / 2011$ & 2,786 & Production & 340 & na & na \\
\hline HR-PR-18 & $9 / 22 / 2011$ & 1,518 & Production & 455 & na & na \\
\hline HR-PR-19 & $10 / 3 / 2011$ & 4,128 & Production & 200 & 31 & 200 \\
\hline HR-PR-20 & $10 / 4 / 2011$ & 933 & Production & 104 & 20 & 104 \\
\hline HR-PR-21 & $10 / 17 / 2011$ & 1,622 & Production & 330 & na & na \\
\hline HR-PR-22 & $10 / 31 / 2011$ & 4,035 & Production & 570 & 0 & 570 \\
\hline HR-PR-23 & $10 / 31 / 2011$ & 3,182 & Production & 703 & 120 & 703 \\
\hline HR-PR-24 & $11 / 1 / 2011$ & 529 & Production & 215 & 100 & 200 \\
\hline HR-PR-25 & $11 / 1 / 2011$ & 2,239 & Production & 280 & 240 & 280 \\
\hline HR-PR-26 & $11 / 2 / 2011$ & 425 & Production & 305 & 282 & 305 \\
\hline HR-PR-27 & $11 / 3 / 2011$ & 3,757 & Production & 186 & 60 & 186 \\
\hline HR-PR-28 & $11 / 14 / 2011$ & 2,866 & Production & 416 & 336 & 416 \\
\hline HR-PR-29 & $11 / 15 / 2011$ & 4,058 & Production & 1,400 & na & na \\
\hline HR-PR-30 & $11 / 16 / 2011$ & 5,172 & Production & 355 & 175 & 335 \\
\hline
\end{tabular}


Table 1. Identification, sampling, and construction information for wells sampled for the Santa Cruz, San Gabriel, and Peninsular Ranges Hard Rock Aquifers (Hard Rock) study unit, March 2011 through March 2012, California GAMA Priority Basin Project.Continued

[GAMA well identification number: HR-SC, Santa Cruz study area grid well; HR-SG, San Gabriel study area grid well; HR-SGU, San Gabriel study area understanding well; HR-PR, Peninsular Ranges study area grid well; HR-PRFP, Peninsular Ranges study area flow-path well; HR-PRU, Peninsular Ranges study area understanding well. Other abbreviations: ft, feet; LSD, land-surface datum; NAVD 88, North American Vertical Datum of 1988; na, not available]

\begin{tabular}{|c|c|c|c|c|c|c|}
\hline $\begin{array}{l}\text { GAMA well } \\
\text { identification } \\
\text { number }\end{array}$ & $\begin{array}{l}\text { Date sampled } \\
\text { (mm/dd/yyyv) }\end{array}$ & $\begin{array}{c}\text { Altitude of LSD } \\
\text { (ft above NAVD 88) }\end{array}$ & Well type & $\begin{array}{c}\text { Well depth } \\
\text { (ft below LSD) }\end{array}$ & $\begin{array}{c}\text { Top of perforated } \\
\text { interval } \\
\text { (ft below LSD) }\end{array}$ & $\begin{array}{c}\text { Bottom of } \\
\text { perforated interval } \\
\text { (ft below LSD) }\end{array}$ \\
\hline \multicolumn{7}{|c|}{ Peninsular Ranges study area (50 wells) —Continued } \\
\hline HR-PR-31 & $11 / 16 / 2011$ & 5,695 & Production & 645 & 50 & 545 \\
\hline HR-PR-32 & $11 / 17 / 2011$ & 3,853 & Production & 310 & 160 & 310 \\
\hline HR-PR-33 & $11 / 28 / 2011$ & 3,562 & Production & na & na & na \\
\hline HR-PR-34 & $11 / 28 / 2011$ & 3,475 & Production & 75 & 30 & 75 \\
\hline HR-PR-35 & $11 / 29 / 2011$ & 3,481 & Production & 1,000 & na & na \\
\hline HR-PR-36 & $11 / 29 / 2011$ & 5,795 & Production & 500 & na & na \\
\hline HR-PRFP-01 & 1/9/2012 & 3,430 & Production & 465 & 50 & 465 \\
\hline HR-PRFP-02 & 1/9/2012 & 876 & Production & na & na & na \\
\hline HR-PRFP-03 & $1 / 10 / 2012$ & 3,265 & Production & na & na & na \\
\hline HR-PRFP-04 & $1 / 10 / 2012$ & 1,536 & Production & 1,600 & na & na \\
\hline HR-PRFP-05 & $1 / 11 / 2012$ & 1,808 & Production & 720 & na & na \\
\hline HR-PRFP-06 & $1 / 11 / 2012$ & 1,996 & Production & 750 & 20 & 750 \\
\hline HR-PRFP-07 & $1 / 12 / 2012$ & 4,145 & Production & 500 & 380 & 500 \\
\hline HR-PRFP-08 & $1 / 24 / 2012$ & 4,109 & Production & 980 & 100 & 980 \\
\hline HR-PRFP-09 & $1 / 25 / 2012$ & 426 & Production & na & na & na \\
\hline HR-PRFP-10 & $1 / 26 / 2012$ & 3,714 & Production & 500 & na & na \\
\hline HR-PRFP-11 & $2 / 21 / 2012$ & 198 & Production & 350 & 305 & 345 \\
\hline HR-PRFP-12 & $2 / 22 / 2012$ & 532 & Production & 128 & 38 & 128 \\
\hline HR-PRFP-13 & 3/8/2012 & 868 & Production & 200 & 0 & 200 \\
\hline HR-PRU-01 & $9 / 20 / 2011$ & 4,931 & Production & 340 & 107 & 340 \\
\hline
\end{tabular}

${ }^{1}$ Land-surface datum (LSD) is a datum plane that is approximately at land surface at each well. The altitude of the LSD is described in feet above the North American Vertical Datum of 1988. 
Table 2. Classes of chemical constituents and field water-quality indicators collected for the Santa Cruz, San Gabriel, and Peninsular Ranges Hard Rock Aquifers (Hard Rock) study unit, March 2011 through March 2012, California GAMA Priority Basin Project.

\begin{tabular}{|c|c|c|}
\hline Constituent classes & Constituent list table & Results table \\
\hline \multicolumn{3}{|l|}{ Field water-quality indicators } \\
\hline Dissolved oxygen, temperature, $\mathrm{pH}$, and specific conductance & none & 4 \\
\hline Field alkalinity, bicarbonate, and carbonate ${ }^{1}$ & none & 4 \\
\hline \multicolumn{3}{|l|}{ Organic constituents } \\
\hline Volatile organic compounds (VOCs) & $3 \mathrm{~A}$ & 5 \\
\hline Pesticides and pesticide degradates & 3B & 6 \\
\hline \multicolumn{3}{|c|}{ Constituent of special interest } \\
\hline Perchlorate & $3 \mathrm{C}$ & 7 \\
\hline \multicolumn{3}{|l|}{ Inorganic constituents } \\
\hline Trace elements & $3 \mathrm{D}$ & 8 \\
\hline Nutrients & $3 \mathrm{E}$ & 9 \\
\hline Major and minor ions, silica, and total dissolved solids (TDS) & $3 \mathrm{~F}$ & 10 \\
\hline Laboratory alkalinity, bicarbonate, and carbonate & $3 \mathrm{~F}$ & 4 \\
\hline Arsenic, chromium, and iron species ${ }^{2}$ & $3 \mathrm{G}$ & 11 \\
\hline \multicolumn{3}{|c|}{ Isotopic tracers, dissolved noble gases, and radioactive constituents } \\
\hline Stable isotopes of hydrogen and oxygen in water & $3 \mathrm{H}$ & 12 \\
\hline Stable isotopes of dissolved boron and strontium in water & $3 \mathrm{H}$ & none $^{3}$ \\
\hline Stable isotopes of carbon in dissolved inorganic carbon and carbon-14 abundance & $3 \mathrm{H}$ & 12 \\
\hline Tritium & $3 \mathrm{H}$ & 12 \\
\hline Stable isotope ratios of helium, dissolved noble gases, and tritium & $3 \mathrm{I}$ & ${ }^{3} 13$ \\
\hline Radon-222 & $3 \mathrm{H}$ & $14 \mathrm{~A}$ \\
\hline Gross alpha and gross beta radioactivity (72-hour and 30-day counts) & $3 \mathrm{H}$ & 14B \\
\hline Uranium isotopes $(234,235 \text {, and } 238)^{4}$ & $3 \mathrm{H}$ & $14 \mathrm{C}$ \\
\hline
\end{tabular}

${ }^{1}$ Field alkalinity was collected at approximately 10 percent of all sampled sites.

${ }^{2}$ Chromium species were collected at sites in the San Gabriel study area only.

${ }^{3}$ Results for stable isotopes of dissolved boron and strontium and for tritium analyzed at the Lawrence Livermore National Laboratory were not completed in time for inclusion in this report; results will be presented in a subsequent publication.

${ }^{4}$ Uranium isotopes only analyzed for samples with uranium concentrations greater than or equal to 10 micrograms per liter. 
Table 3A. Volatile organic compounds (VOCs), primary uses or sources, reporting information, and comparative benchmarks for the U.S. Geological Survey (USGS) National Water Quality Laboratory Schedule 2020.

[The five-digit USGS parameter code is used to uniquely identify a specific constituent or property. Reporting levels, benchmark types, and benchmark levels as of July 6, 2012. Benchmark type: Maximum contaminant levels are listed as MCL-US when the MCL-US and MCL-CA are identical, and as MCL-CA when the MCL-CA is lower than the MCL-US or no MCL-US exists. HAL-US, U.S. Environmental Protection Agency (USEPA) lifetime health advisory level; MCL-CA, California Department of Public Health (CDPH) maximum contaminant level; MCL-US, USEPA maximum contaminant level; NL-CA, CDPH notification level; RSD5-US, USEPA risk-specific dose at a risk factor of $10^{-5}$. Other abbreviations: CAS, Chemical Abstracts Service; LRL, laboratory reporting level; $\mu \mathrm{g} / \mathrm{L}$, micrograms per liter; THM, trihalomethane; na, not available; —, not detected; D, detected in groundwater samples (table 5)]

\begin{tabular}{|c|c|c|c|c|c|c|c|}
\hline $\begin{array}{c}\text { Constituent } \\
\text { (synonym or abbreviation) }\end{array}$ & Primary use or source & $\begin{array}{c}\text { USGS } \\
\text { parameter } \\
\text { code }\end{array}$ & $\begin{array}{l}\text { CAS registry } \\
\text { number }^{\circledR 1}\end{array}$ & $\begin{array}{c}\text { LRL } \\
(\mu \mathrm{g} / \mathrm{L})^{2}\end{array}$ & $\begin{array}{l}\text { Benchmark } \\
\text { type }\end{array}$ & $\begin{array}{c}\text { Benchmark } \\
\text { level } \\
(\mu \mathrm{g} / \mathrm{L})\end{array}$ & Detection \\
\hline Acetone & Solvent & 81552 & $67-64-1$ & 3.4 & na & na & -3 \\
\hline Acrylonitrile & Organic synthesis & 34215 & $107-13-1$ & $0.8,0.48$ & RSD5-US & 0.6 & - \\
\hline tert-Amyl methyl ether (TAME) & Gasoline oxygenate & 50005 & $994-05-8$ & 0.06 & na & na & - \\
\hline Benzene & Gasoline hydrocarbon & 34030 & $71-43-2$ & 0.026 & MCL-CA & 1 & - \\
\hline Bromobenzene & Solvent & 81555 & $108-86-1$ & 0.022 & HAL-US & 70 & - \\
\hline Bromochloromethane & Fire retardant & 77297 & $74-97-5$ & 0.06 & HAL-US & 90 & $\mathrm{D}$ \\
\hline Bromodichloromethane & Disinfection byproduct (THM) & 32101 & $75-27-4$ & 0.034 & MCL-US & ${ }^{4} 80$ & $\mathrm{D}$ \\
\hline Bromoform (Tribromomethane) & Disinfection byproduct (THM) & 32104 & $75-25-2$ & 0.1 & MCL-US & ${ }^{4} 80$ & $\mathrm{D}$ \\
\hline Bromomethane (Methyl bromide) & Fumigant & 34413 & $74-83-9$ & 0.20 & HAL-US & 10 & - \\
\hline$n$-Butylbenzene & Gasoline hydrocarbon & 77342 & $104-51-8$ & 0.08 & NL-CA & 260 & - \\
\hline sec-Butylbenzene & Gasoline hydrocarbon & 77350 & $135-98-8$ & 0.034 & NL-CA & 260 & - \\
\hline tert-Butylbenzene & Gasoline hydrocarbon & 77353 & $98-06-6$ & 0.060 & NL-CA & 260 & - \\
\hline Carbon disulfide & Organic synthesis & 77041 & $75-15-0$ & $0.08,0.1$ & NL-CA & 160 & $\mathrm{D}$ \\
\hline Carbon tetrachloride (Tetrachloromethane) & Solvent & 32102 & $56-23-5$ & 0.06 & MCL-CA & 0.5 & $\mathrm{D}$ \\
\hline Chlorobenzene & Solvent & 34301 & $108-90-7$ & 0.026 & MCL-CA & 70 & $\mathrm{D}$ \\
\hline Chloroethane & Solvent & 34311 & $75-00-3$ & 0.06 & na & na & $\mathrm{D}$ \\
\hline Chloroform (Trichloromethane) & Disinfection byproduct (THM) & 32106 & $67-66-3$ & 0.03 & MCL-US & ${ }^{4} 80$ & $\mathrm{D}$ \\
\hline Chloromethane & Solvent & 34418 & $74-87-3$ & $0.14,0.1$ & na & na & $\mathrm{D}$ \\
\hline 3-Chloropropene & Organic synthesis & 78109 & $107-05-1$ & 0.08 & na & na & - \\
\hline 2-Chlorotoluene & Solvent & 77275 & $95-49-8$ & 0.028 & NL-CA & 140 & - \\
\hline 4-Chlorotoluene & Solvent & 77277 & $106-43-4$ & 0.042 & NL-CA & 140 & - \\
\hline Dibromochloromethane & Disinfection byproduct (THM) & 32105 & $124-48-1$ & 0.12 & MCL-US & ${ }^{4} 80$ & $\mathrm{D}$ \\
\hline 1,2-Dibromo-3-chloropropane (DBCP) & Fumigant & 82625 & $96-12-8$ & 0.40 & MCL-US & 0.2 & - \\
\hline 1,2-Dibromoethane (EDB) & Fumigant & 77651 & $106-93-4$ & 0.028 & MCL-US & 0.05 & - \\
\hline Dibromomethane & Solvent & 30217 & $74-95-3$ & 0.050 & na & na & $\mathrm{D}$ \\
\hline 1,2-Dichlorobenzene & Solvent & 34536 & $95-50-1$ & 0.028 & MCL-CA & 600 & - \\
\hline 1,3-Dichlorobenzene & Solvent & 34566 & $541-73-1$ & 0.024 & HAL-US & 600 & $\mathrm{D}$ \\
\hline
\end{tabular}


Table 3A. Volatile organic compounds (VOCs), primary uses or sources, reporting information, and comparative benchmarks for the U.S. Geological Survey (USGS) National Water Quality Laboratory Schedule 2020.-Continued

[The five-digit USGS parameter code is used to uniquely identify a specific constituent or property. Reporting levels, benchmark types, and benchmark levels as of July 6, 2012. Benchmark type: Maximum contaminant levels are listed as MCL-US when the MCL-US and MCL-CA are identical, and as MCL-CA when the MCL-CA is lower than the MCL-US or no MCL-US exists. HAL-US, U.S. Environmental Protection Agency (USEPA) lifetime health advisory level; MCL-CA, California Department of Public Health (CDPH) maximum contaminant level; MCL-US, USEPA maximum contaminant level; NL-CA, CDPH notification level; RSD5-US, USEPA risk-specific dose at a risk factor of $10^{-5}$. Other abbreviations: CAS, Chemical Abstracts Service; LRL, laboratory reporting level; $\mu \mathrm{g} / \mathrm{L}$, micrograms per liter; THM, trihalomethane; na, not available; - , not detected; D, detected in groundwater samples (table 5)]

\begin{tabular}{|c|c|c|c|c|c|c|c|}
\hline $\begin{array}{c}\text { Constituent } \\
\text { (synonym or abbreviation) }\end{array}$ & Primary use or source & $\begin{array}{c}\text { USGS } \\
\text { parameter } \\
\text { code }\end{array}$ & $\begin{array}{l}\text { CAS registry } \\
\text { number }^{\circledR 1}\end{array}$ & $\begin{array}{c}\text { LRL } \\
(\mu g / L)^{2}\end{array}$ & $\begin{array}{l}\text { Benchmark } \\
\text { type }\end{array}$ & $\begin{array}{c}\text { Benchmark } \\
\text { level } \\
(\mu \mathrm{g} / \mathrm{L})\end{array}$ & Detection \\
\hline 1,4-Dichlorobenzene & Solvent & 34571 & $106-46-7$ & 0.026 & MCL-CA & 5 & $\mathrm{D}$ \\
\hline trans-1,4-Dichloro-2-butene & Organic synthesis & 73547 & $110-57-6$ & $0.36,2$ & na & na & - \\
\hline Dichlorodifluoromethane (CFC-12) & Refrigerant & 34668 & $75-71-8$ & 0.1 & NL-CA & 1,000 & - \\
\hline 1,1-Dichloroethane (1,1-DCA) & Solvent & 34496 & $75-34-3$ & 0.044 & MCL-CA & 5 & - \\
\hline 1,2-Dichloroethane (1,2-DCA) & Solvent & 32103 & $107-06-2$ & 0.08 & MCL-CA & 0.5 & - \\
\hline 1,1-Dichloroethene (1,1-DCE) & Organic synthesis & 34501 & $75-35-4$ & 0.022 & MCL-CA & 6 & $\mathrm{D}$ \\
\hline cis-1,2-Dichloroethene (cis-1,2-DCE) & Solvent & 77093 & $156-59-2$ & 0.022 & MCL-CA & 6 & - \\
\hline trans-1,2-Dichloroethene (trans-1,2-DCE) & Solvent & 34546 & $156-60-5$ & 0.018 & MCL-CA & 10 & - \\
\hline 1,2-Dichloropropane & Fumigant & 34541 & $78-87-5$ & 0.026 & MCL-US & 5 & - \\
\hline 1,3-Dichloropropane & Fumigant & 77173 & $142-28-9$ & 0.06 & na & na & - \\
\hline 2,2-Dichloropropane & Fumigant & 77170 & $594-20-7$ & 0.06 & na & na & - \\
\hline 1,1-Dichloropropene & Organic synthesis & 77168 & $563-58-6$ & 0.040 & na & na & - \\
\hline cis-1,3-Dichloropropene & Fumigant & 34704 & 10061-01-5 & 0.1 & RSD5-US & ${ }^{5} 4$ & - \\
\hline trans-1,3-Dichloropropene & Fumigant & 34699 & $10061-02-6$ & 0.14 & RSD5-US & ${ }^{5} 4$ & - \\
\hline Diethyl ether & Solvent & 81576 & $60-29-7$ & 0.1 & na & na & - \\
\hline Diisopropyl ether (DIPE) & Gasoline oxygenate & 81577 & $108-20-3$ & 0.06 & na & na & - \\
\hline Ethylbenzene & Gasoline hydrocarbon & 34371 & $100-41-4$ & 0.036 & MCL-CA & 300 & - \\
\hline Ethyl tert-butyl ether (ETBE) & Gasoline oxygenate & 50004 & $637-92-3$ & 0.032 & na & na & - \\
\hline Ethyl methacrylate & Organic synthesis & 73570 & $97-63-2$ & 0.20 & na & na & - \\
\hline$o$-Ethyl toluene (1-Ethyl-2-methyl benzene) & Gasoline hydrocarbon & 77220 & $611-14-3$ & 0.032 & na & na & - \\
\hline Hexachlorobutadiene & Organic synthesis & 39702 & $87-68-3$ & 0.08 & RSD5-US & 9 & - \\
\hline Hexachloroethane & Solvent & 34396 & $67-72-1$ & $0.22,0.1$ & HAL-US & 1 & - \\
\hline 2-Hexanone ( $n$-Butyl methyl ketone) & Solvent & 77103 & $591-78-6$ & 0.4 & na & na & - \\
\hline Iodomethane (Methyl iodide) & Organic synthesis & 77424 & $74-88-4$ & 0.26 & na & na & - \\
\hline Isopropylbenzene & Gasoline hydrocarbon & 77223 & $98-82-8$ & 0.042 & NL-CA & 770 & - \\
\hline 4-Isopropyl-1-methyl benzene & Gasoline hydrocarbon & 77356 & $99-87-6$ & 0.06 & na & na & - \\
\hline Methyl acrylate & Organic synthesis & 49991 & $96-33-3$ & 0.8 & na & na & - \\
\hline
\end{tabular}


Table 3A. Volatile organic compounds (VOCs), primary uses or sources, reporting information, and comparative benchmarks for the U.S. Geological Survey (USGS) National Water Quality Laboratory Schedule 2020.-Continued

[The five-digit USGS parameter code is used to uniquely identify a specific constituent or property. Reporting levels, benchmark types, and benchmark levels as of July 6, 2012. Benchmark type: Maximum contaminant levels are listed as MCL-US when the MCL-US and MCL-CA are identical, and as MCL-CA when the MCL-CA is lower than the MCL-US or no MCL-US exists. HAL-US, U.S. Environmental Protection Agency (USEPA) lifetime health advisory level; MCL-CA, California Department of Public Health (CDPH) maximum contaminant level; MCL-US, USEPA maximum contaminant level; NL-CA, CDPH notification level; RSD5-US, USEPA risk-specific dose at a risk factor of $10^{-5}$. Other abbreviations: CAS, Chemical Abstracts Service; LRL, laboratory reporting level; $\mu \mathrm{g} / \mathrm{L}$, micrograms per liter; THM, trihalomethane; na, not available; —, not detected; D, detected in groundwater samples (table 5)]

\begin{tabular}{|c|c|c|c|c|c|c|c|}
\hline $\begin{array}{c}\text { Constituent } \\
\text { (synonym or abbreviation) }\end{array}$ & Primary use or source & $\begin{array}{c}\text { USGS } \\
\text { parameter } \\
\text { code }\end{array}$ & $\begin{array}{l}\text { CAS registry } \\
\text { number }^{\circledR 1}\end{array}$ & $\begin{array}{c}\text { LRL } \\
(\mu \mathrm{g} / \mathrm{L})^{2}\end{array}$ & $\begin{array}{l}\text { Benchmark } \\
\text { type }\end{array}$ & $\begin{array}{c}\text { Benchmark } \\
\text { level } \\
(\mu \mathrm{g} / \mathrm{L})\end{array}$ & Detection \\
\hline Methyl acrylonitrile & Organic synthesis & 81593 & $126-98-7$ & 0.26 & na & na & - \\
\hline Methyl tert-butyl ether (MTBE) & Gasoline oxygenate & 78032 & $1634-04-4$ & 0.1 & MCL-CA & 13 & $\mathrm{D}$ \\
\hline Methyl iso-butyl ketone (MIBK) & Solvent & 78133 & $108-10-1$ & 0.32 & NL-CA & 120 & - \\
\hline Methyl ethyl ketone (2-butanone, MEK) & Solvent & 81595 & $78-93-3$ & 1.6 & HAL-US & 4,000 & -3 \\
\hline Methyl methacrylate & Organic synthesis & 81597 & $80-62-6$ & 0.22 & na & na & - \\
\hline Methylene chloride (Dichloromethane) & Solvent & 34423 & $75-09-2$ & 0.04 & MCL-US & 5 & $\mathrm{D}$ \\
\hline Naphthalene & Gasoline hydrocarbon & 34696 & $91-20-3$ & 0.18 & NL-CA & 17 & - \\
\hline Perchloroethene (PCE, Tetrachloroethene) & Solvent & 34475 & $127-18-4$ & 0.026 & MCL-US & 5 & $\mathrm{D}$ \\
\hline$n$-Propylbenzene & Solvent & 77224 & $103-65-1$ & 0.036 & NL-CA & 260 & - \\
\hline Styrene & Gasoline hydrocarbon & 77128 & $100-42-5$ & 0.042 & MCL-US & 100 & - \\
\hline 1,1,1,2-Tetrachloroethane & Solvent & 77562 & $630-20-6$ & 0.040 & HAL-US & 70 & - \\
\hline 1,1,2,2-Tetrachloroethane & Solvent & 34516 & $79-34-5$ & 0.14 & MCL-CA & 1 & - \\
\hline Tetrahydrofuran & Solvent & 81607 & $109-99-9$ & 1.4 & na & na & -3 \\
\hline 1,2,3,4-Tetramethylbenzene & Gasoline hydrocarbon & 49999 & $488-23-3$ & 0.1 & na & na & - \\
\hline 1,2,3,5-Tetramethylbenzene & Gasoline hydrocarbon & 50000 & $527-53-7$ & 0.08 & na & na & - \\
\hline Toluene & Gasoline hydrocarbon & 34010 & $108-88-3$ & $0.018,0.02$ & MCL-CA & 150 & $\mathrm{D}$ \\
\hline 1,2,3-Trichlorobenzene & Organic synthesis & 77613 & $87-61-6$ & 0.06 & na & na & $\mathrm{D}$ \\
\hline 1,2,4-Trichlorobenzene & Solvent & 34551 & $120-82-1$ & 0.08 & MCL-CA & 5 & $\mathrm{D}$ \\
\hline $1,1,1$-Trichloroethane $(1,1,1$-TCA) & Solvent & 34506 & $71-55-6$ & 0.030 & MCL-US & 200 & $\mathrm{D}$ \\
\hline 1,1,2-Trichloroethane (1,1,2-TCA) & Solvent & 34511 & $79-00-5$ & $0.028,0.046$ & MCL-US & 5 & - \\
\hline Trichloroethene (TCE) & Solvent & 39180 & $79-01-6$ & 0.022 & MCL-US & 5 & $\mathrm{D}$ \\
\hline Trichlorofluoromethane (CFC-11) & Refrigerant & 34488 & $75-69-4$ & 0.06 & MCL-CA & 150 & $\mathrm{D}$ \\
\hline 1,2,3-Trichloropropane (1,2,3-TCP) & Solvent/organic synthesis & 77443 & $96-18-4$ & 0.12 & HAL-US ${ }^{6}$ & 40 & - \\
\hline Trichlorotrifluoroethane (CFC-113) & Refrigerant & 77652 & $76-13-1$ & $0.034,0.022$ & MCL-CA & 1,200 & $\mathrm{D}$ \\
\hline 1,2,3-Trimethylbenzene & Gasoline hydrocarbon & 77221 & $526-73-8$ & 0.060 & na & na & - \\
\hline 1,2,4-Trimethylbenzene & Gasoline hydrocarbon & 77222 & $95-63-6$ & 0.032 & NL-CA & 330 & -3 \\
\hline 1,3,5-Trimethylbenzene & Organic synthesis & 77226 & $108-67-8$ & 0.032 & NL-CA & 330 & - \\
\hline
\end{tabular}


Table 3A. Volatile organic compounds (VOCs), primary uses or sources, reporting information, and comparative benchmarks for the U.S. Geological Survey (USGS) National Water Quality Laboratory Schedule 2020.-Continued

[The five-digit USGS parameter code is used to uniquely identify a specific constituent or property. Reporting levels, benchmark types, and benchmark levels as of July 6, 2012. Benchmark type: Maximum contaminant levels are listed as MCL-US when the MCL-US and MCL-CA are identical, and as MCL-CA when the MCL-CA is lower than the MCL-US or no MCL-US exists. HAL-US, U.S. Environmenta Protection Agency (USEPA) lifetime health advisory level; MCL-CA, California Department of Public Health (CDPH) maximum contaminant level; MCL-US, USEPA maximum contaminant level; NL-CA, CDPH notification level; RSD5-US, USEPA risk-specific dose at a risk factor of $10^{-5}$. Other abbreviations: CAS, Chemical Abstracts Service; LRL, laboratory reporting level; $\mu \mathrm{g} / \mathrm{L}$, micrograms per liter; THM, trihalomethane; na, not available; - , not detected; D, detected in groundwater samples (table 5)]

\begin{tabular}{|c|c|c|c|c|c|c|c|}
\hline $\begin{array}{c}\text { Constituent } \\
\text { (synonym or abbreviation) }\end{array}$ & Primary use or source & $\begin{array}{c}\text { USGS } \\
\text { parameter } \\
\text { code }\end{array}$ & $\begin{array}{l}\text { CAS registry } \\
\text { number }^{\circledR 1}\end{array}$ & $\begin{array}{c}\text { LRL } \\
(\mu \mathrm{g} / \mathrm{L})^{2}\end{array}$ & $\begin{array}{l}\text { Benchmark } \\
\text { type }\end{array}$ & $\begin{array}{c}\text { Benchmark } \\
\text { level } \\
(\mu \mathrm{g} / \mathrm{L}) \\
\end{array}$ & Detection \\
\hline Vinyl bromide (Bromoethene) & Fire retardant & 50002 & $593-60-2$ & 0.12 & na & na & - \\
\hline Vinyl chloride (Chloroethene) & Organic synthesis & 39175 & $75-01-4$ & 0.06 & MCL-CA & 0.5 & - \\
\hline$m$ - and $p$-Xylene & Gasoline hydrocarbon & 85795 & $108-38-3 / 106-42-3$ & 0.08 & MCL-CA & ${ }^{7} 1,750$ & - \\
\hline$o$-Xylene & Gasoline hydrocarbon & 77135 & $95-47-6$ & 0.032 & MCL-CA & ${ }^{7} 1,750$ & - \\
\hline
\end{tabular}

${ }^{1}$ This report contains CAS Registry Numbers ${ }^{\circledR}$ (CASRNs), which is a Registered Trademark of the American Chemical Society. CAS recommends the verification of the CASRNs through CAS Client Services ${ }^{\mathrm{sM}}$.

${ }^{2}$ For constituents with two LRLs listed, the first value was in use before October 1, 2011, and the second value was in use starting October 1, 2011. The higher LRL is used for this report.

${ }^{3}$ Study reporting levels (SRLs) determined on the basis of the quality-control data collected between May 2004 and September 2010 for the first 32 study uints of the California Groundwater Ambient Monitoring and Assessment (GAMA) Priority Basin Project (Fram and others, 2012) resulted in censoring all groundwater detections when applied to four VOCs for the Hard Rock study unit. All detections of acetone, 2-butanone, and tetrahydrofuran were censored from this dataset and coded in the USGS database as "reviewed and rejected." All groundwater detections of 1,2,4 -trimethylbenzene were re-classified as nondetections and are reported with the following comment in the USGS National Water Information System database: Result is $<$ or $=$ reported value, based on quality-control data (Fram and others, 2012). See appendix table A3 for more information.

${ }^{4}$ The MCL-US benchmark for trihalomethanes is for the sum of chloroform, bromoform, bromodichloromethane, and dibromochloromethane.

${ }^{5}$ The RSD5 benchmark for 1,3-dichloropropene is for the sum of its isomers (cis and trans).

${ }^{6}$ In earlier reports in this series, the NL-CA $(0.005 \mu \mathrm{g} / \mathrm{L})$ was used as the comparative benchmark for 1,2,3-TCP.

${ }^{7}$ The MCL-CA benchmark for $m$ - and $p$-xylene and $o$-xylene is for the sum of all three xylene compounds. 
Table 3B. Pesticides and pesticide degradates, primary uses or sources, reporting information, and comparative benchmarks for the U.S. Geological Survey (USGS) National Water Quality Laboratory Schedule 2003.

[The five-digit USGS parameter code is used to uniquely identify a specific constituent or property. Reporting levels, benchmark types, and benchmark levels as of July 6, 2012. Benchmark type: Maximum contaminant levels are listed as MCL-US when the MCL-US and MCL-CA are identical, and as MCL-CA when the MCL-CA is lower than the MCL-US or no MCL-US exists. HAL-US, U.S. Environmental Protection Agency (USEPA) lifetime health advisory level; MCL-CA, California Department of Public Health maximum contaminant level; MCL-US, USEPA maximum contaminant level; RSD5-US, USEPA riskspecific dose at a risk factor of $10^{-5}$. Other abbreviations: CAS, Chemical Abstracts Service; LRL, laboratory reporting level; $\mu \mathrm{g} / \mathrm{L}$, micrograms per liter; na, not available; - , not detected; D, detected in groundwater samples (table 6)]

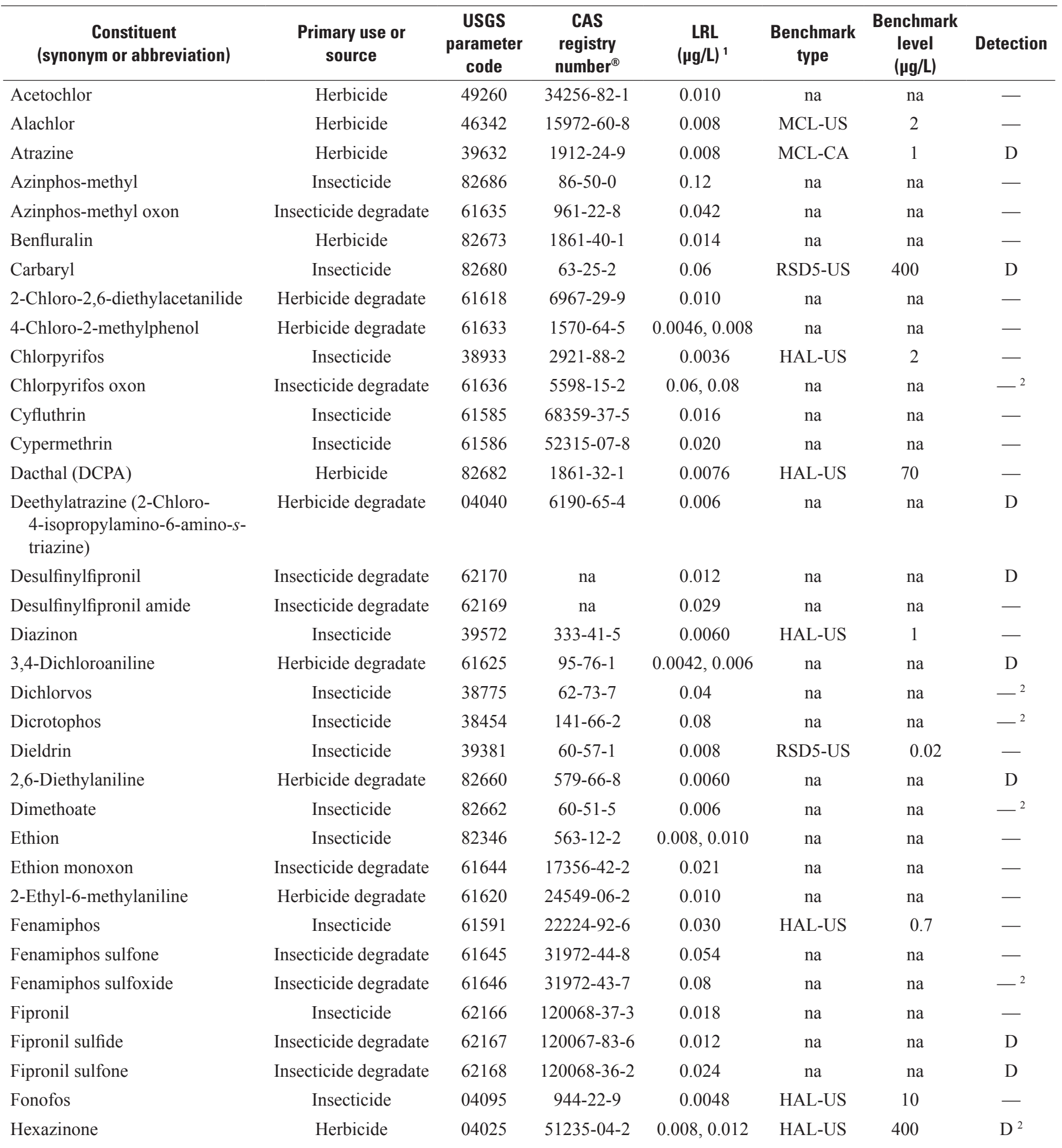


Table 3B. Pesticides and pesticide degradates, primary uses or sources, reporting information, and comparative benchmarks for the U.S. Geological Survey (USGS) National Water Quality Laboratory Schedule 2003._-Continued

[The five-digit USGS parameter code is used to uniquely identify a specific constituent or property. Reporting levels, benchmark types, and benchmark levels as of July 6, 2012. Benchmark type: Maximum contaminant levels are listed as MCL-US when the MCL-US and MCL-CA are identical, and as MCL-CA when the MCL-CA is lower than the MCL-US or no MCL-US exists. HAL-US, U.S. Environmental Protection Agency (USEPA) lifetime health advisory level; MCL-CA, California Department of Public Health maximum contaminant level; MCL-US, USEPA maximum contaminant level; RSD5-US, USEPA riskspecific dose at a risk factor of $10^{-5}$. Other abbreviations: CAS, Chemical Abstracts Service; LRL, laboratory reporting level; $\mu \mathrm{g} / \mathrm{L}$, micrograms per liter; na, not available; - , not detected; D, detected in groundwater samples (table 6)]

\begin{tabular}{|c|c|c|c|c|c|c|c|}
\hline $\begin{array}{c}\text { Constituent } \\
\text { (synonym or abbreviation) }\end{array}$ & $\begin{array}{l}\text { Primary use or } \\
\text { source }\end{array}$ & $\begin{array}{c}\text { USGS } \\
\text { parameter } \\
\text { code }\end{array}$ & $\begin{array}{c}\text { CAS } \\
\text { registry } \\
\text { number }^{\circledR}\end{array}$ & $\begin{array}{c}\text { LRL } \\
(\mu \mathrm{g} / \mathrm{L})^{1}\end{array}$ & $\begin{array}{l}\text { Benchmark } \\
\text { type }\end{array}$ & $\begin{array}{c}\text { Benchmark } \\
\text { level } \\
\text { ( } \mu \mathrm{g} / \mathrm{L})\end{array}$ & Detection \\
\hline Iprodione & Fungicide & 61593 & $36734-19-7$ & 0.014 & na & na & - \\
\hline Isofenphos & Insecticide & 61594 & 25311-71-1 & $0.006,0.008$ & na & na & - \\
\hline Malaoxon & Insecticide degradate & 61652 & $1634-78-2$ & 0.022 & na & na & - \\
\hline Metalaxyl & Fungicide & 61596 & $57837-19-1$ & 0.014 & na & na & - \\
\hline Methidathion & Insecticide & 61598 & $950-37-8$ & 0.012 & na & na & - \\
\hline Metolachlor & Herbicide & 39415 & $51218-45-2$ & 0.020 & HAL-US & 700 & $\mathrm{D}$ \\
\hline Metribuzin & Herbicide & 82630 & 21087-64-9 & 0.012 & HAL-US & 70 & $\mathrm{D}$ \\
\hline Parathion-methyl & Insecticide & 82667 & $298-00-0$ & 0.008 & HAL-US & 1 & - \\
\hline Pendimethalin & Herbicide & 82683 & $40487-42-1$ & 0.012 & na & na & - \\
\hline cis-Permethrin & Insecticide & 82687 & $54774-45-7$ & 0.010 & na & na & - \\
\hline Phorate & Insecticide & 82664 & $298-02-2$ & 0.020 & na & na & - \\
\hline Phorate oxon & Insecticide degradate & 61666 & $2600-69-3$ & 0.027 & na & na & - \\
\hline Phosmet & Insecticide & 61601 & $732-11-6$ & $0.14,0.08$ & na & na & -2 \\
\hline Phosmet oxon & Insecticide degradate & 61668 & $3735-33-9$ & 0.0511 & na & na & -2 \\
\hline Prometon & Herbicide & 04037 & 1610-18-0 & 0.012 & HAL-US & 400 & $\mathrm{D}$ \\
\hline Terbufos oxon sulfone & Insecticide degradate & 61674 & $56070-15-6$ & 0.045 & na & na & -2 \\
\hline Terbuthylazine & Herbicide & 04022 & $5915-41-3$ & $0.0060,0.008$ & na & na & - \\
\hline Tribufos & Defoliant & 61610 & $78-48-8$ & 0.018 & na & na & -2 \\
\hline Trifluralin & Herbicide & 82661 & $1582-09-8$ & 0.018 & HAL-US & 10 & - \\
\hline
\end{tabular}

${ }^{1}$ For constituents with two LRLs listed, the first value was in use before October 1, 2011, and the second value was in use starting October 1, 2011. The highest LRL is used for this report.

${ }^{2}$ The median matrix-spike recovery was less than 70 percent. Low recoveries may indicate that the compound might not have been detected in some samples if it was present at very low concentrations.

${ }^{3}$ The median matrix-spike recovery was greater than 130 percent. High recoveries may indicate that the reported concentrations were greater than what were in the samples. 
Table 3C. Constituent of special interest, primary uses or sources, reporting information, and comparative benchmark for analysis performed by Weck Laboratories, Inc.

[The five-digit U.S. Geological Survey (USGS) parameter code is used to uniquely identify a specific constituent or property. Reporting level, benchmark type, and benchmark level as of July 6, 2012. Benchmark type: Maximum contaminant levels are listed as MCL-US when the MCL-US and MCL-CA are identical, and as MCL-CA when the MCL-CA is lower than the MCL-US or no MCL-US exists. MCL-US, USEPA maximum contaminant level; MCL-CA, California Department of Public Health (CDPH) maximum contaminant level. Other abbreviations: CAS, Chemical Abstracts Service; MRL, minimum reporting level; $\mu \mathrm{g} / \mathrm{L}$, micrograms per liter; $\mathrm{D}$, detected in groundwater samples (table 7)]

\begin{tabular}{|c|c|c|c|c|c|c|c|}
\hline Constituent & Primary uses or sources & $\begin{array}{c}\text { USGS } \\
\text { parameter } \\
\text { code }\end{array}$ & $\begin{array}{c}\text { CAS } \\
\text { registry } \\
\text { number }^{\circledR}\end{array}$ & $\begin{array}{c}\text { MRL } \\
(\mu \mathrm{g} / \mathrm{L})\end{array}$ & $\begin{array}{c}\text { Benchmark } \\
\text { type }\end{array}$ & $\begin{array}{c}\text { Benchmark } \\
\text { level } \\
\text { ( } \mu \mathrm{g} / \mathrm{L})\end{array}$ & Detection \\
\hline Perchlorate & Rocket fuel, fireworks, flares & 63790 & $14797-73-0$ & 0.10 & MCL-CA & 6 & $\mathrm{D}$ \\
\hline
\end{tabular}

Table 3D. Trace elements, reporting information, and comparative benchmarks for the U.S. Geological Survey (USGS) National Water Quality Laboratory Schedule 1948.

[The five-digit USGS parameter code is used to uniquely identify a specific constituent or property. Reporting levels, benchmark types, and benchmark levels as of July 6, 2012. Benchmark type: Maximum contaminant levels are listed as MCL-US when the MCL-US and MCL-CA are identical, and as MCL-CA when the MCL-CA is lower than the MCL-US or no MCL-US exists. Secondary maximum contaminant level benchmarks are listed as SMCL-CA when the SMCL-CA exists, and as the SMCL-US when no SMCL-CA exists. AL-US, U.S. Environmental Protection Agency (USEPA) action level; HAL-US, USEPA lifetime health advisory level; MCL-CA, California Department of Public Health (CDPH) maximum contaminant level; MCL-US, USEPA maximum contaminant level; NL-CA, CDPH notification level; SMCL-CA, CDPH secondary maximum contaminant level. Other abbreviations: CAS, Chemical Abstracts Service; LT-MDL, long-term method detection level; na, not available; $\mu \mathrm{g} / \mathrm{L}$, micrograms per liter; D, detected in groundwater samples (table 8)]

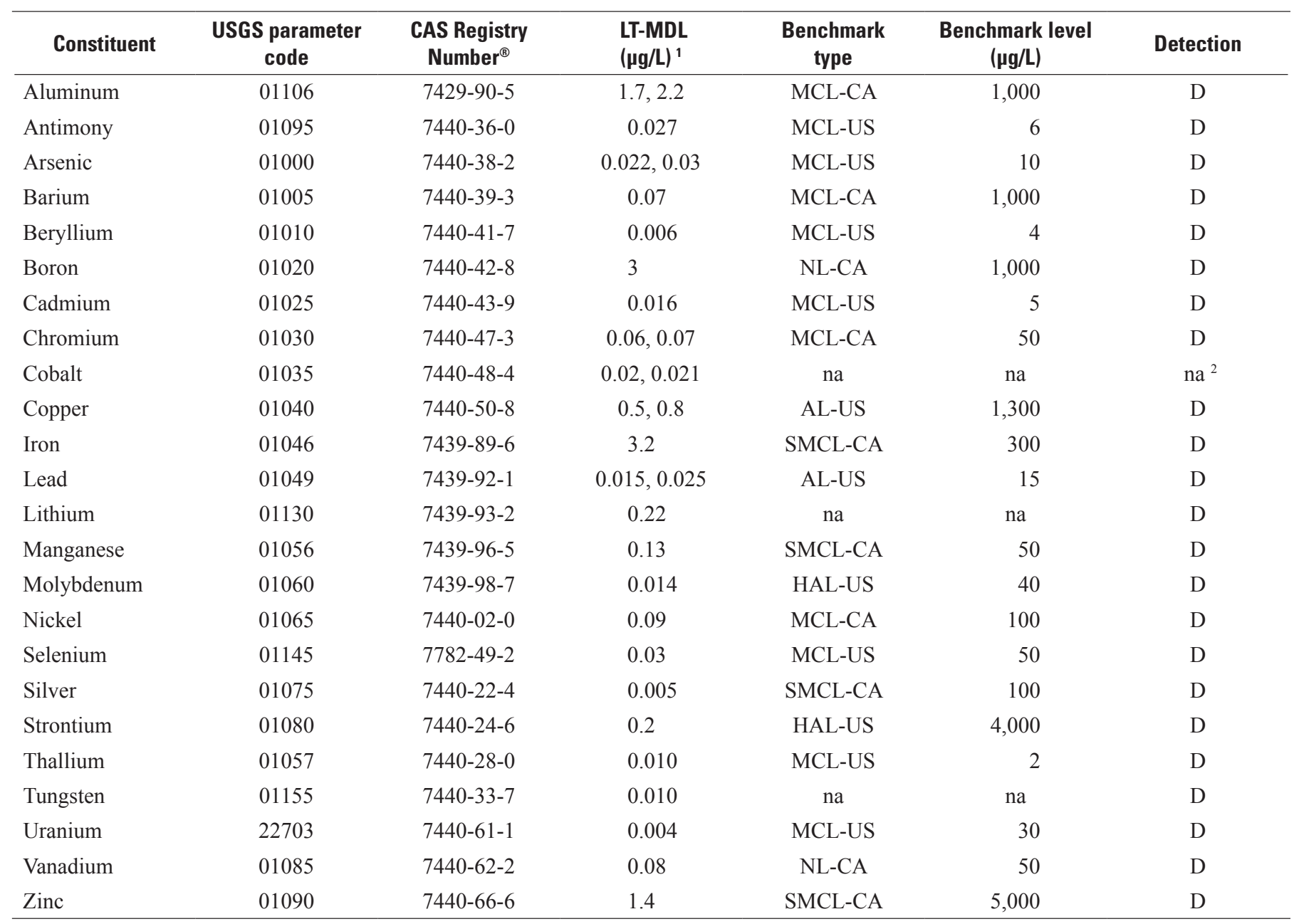

${ }^{1}$ For constituents with two LT-MDLs listed, the first value was in use before October 1, 2011, and the second value was in use starting October 1, 2011. The highest LT-MDL is used for this report.

${ }^{2}$ Evaluation of quality-control data collected from October 2009 through March 2013 for the California Groundwater Ambient Monitoring and Assessment (GAMA) Priority Basin Project resulted in censoring all groundwater results for cobalt for the Hard Rock study unit (Davis and others, 2014). All results for cobalt were censored from this dataset and coded in the USGS database as "reviewed and rejected." See appendix table A3 for more information. 
Table 3E. Nutrients, reporting information, and comparative benchmarks for the U.S. Geological Survey (USGS) National Water Quality Laboratory Schedule 2755.

[The five-digit USGS parameter code is used to uniquely identify a specific constituent or property. Reporting levels, benchmark types, and benchmark levels as of July 6, 2012. Benchmark type: HAL-US, U.S. Environmental Protection Agency (USEPA) lifetime health advisory level; MCL-US, USEPA maximum contaminant level. Other abbreviations: CAS, Chemical Abstracts Service; LT-MDL, long-term method detection level; MDL, method detection limit; mg/L, milligrams per liter; na, not available; D, detected in groundwater samples (table 9)]

\begin{tabular}{|c|c|c|c|c|c|c|}
\hline Constituent & $\begin{array}{c}\text { USGS } \\
\text { parameter } \\
\text { code }\end{array}$ & $\begin{array}{c}\text { CAS } \\
\text { registry } \\
\text { number }^{\circledR}\end{array}$ & $\begin{array}{l}\text { LT-MDL } \\
\text { or MDL } \\
\text { (mg/L) }\end{array}$ & $\begin{array}{l}\text { Benchmark } \\
\text { type }\end{array}$ & $\begin{array}{c}\text { Benchmark } \\
\text { level } \\
\text { (mg/L) }\end{array}$ & Detection \\
\hline Ammonia (as nitrogen) & 00608 & $7664-41-7$ & 0.010 & HAL-US & ${ }^{1} 24.7$ & $\mathrm{D}$ \\
\hline Nitrate plus nitrite (as nitrogen) & 00631 & na & ${ }^{2} 0.02,0.04$ & MCL-US & 10 & $\mathrm{D}$ \\
\hline Nitrite (as nitrogen) & 00613 & $14797-65-0$ & 0.0010 & MCL-US & 1 & $\mathrm{D}$ \\
\hline $\begin{array}{l}\text { Total nitrogen (ammonia, nitrite, nitrate, } \\
\text { organic nitrogen) }\end{array}$ & 62854 & $17778-88-0$ & 0.05 & na & na & $\mathrm{D}$ \\
\hline Phosphate, orthophosphate (as phosphorus) & 00671 & $14265-44-2$ & 0.004 & na & na & $\mathrm{D}$ \\
\hline
\end{tabular}

${ }^{1}$ The HAL-US is $30 \mathrm{mg} / \mathrm{L}$ "as ammonia." To facilitate comparson to the analytical results, we have converted and reported this HAL-US as $24.7 \mathrm{mg} / \mathrm{L}$ "as nitrogen."

${ }^{2}$ For nitrate plus nitrite, the first value was the MDL in use before October 17, 2011, and the second value was the interim reporting level (IRL) in use starting October 17, 2011.

Table 3F. Major and minor ions, silica, total dissolved solids (TDS), and laboratory alkalinity, reporting information, and comparative benchmarks for the U.S. Geological Survey (USGS) National Water Quality Laboratory Schedule 1948.

[The five-digit USGS parameter code is used to uniquely identify a specific constituent or property. Reporting levels, benchmark types, and benchmark levels as of July 6, 2012. Benchmark type: Maximum contaminant levels are listed as MCL-US when the MCL-US and MCL-CA are identical, and as MCL-CA when the MCL-CA is lower than the MCL-US or no MCL-US exists. Secondary maximum contaminant level benchmarks are listed as SMCL-CA when the SMCL-CA exists, and as the SMCL-US when no SMCL-CA exists. MCL-CA, California Department of Public Health (CDPH) maximum contaminant level; SMCL-CA, CDPH secondary maximum contaminant level. Other abbreviations: CAS, Chemical Abstracts Service; LT-MDL, long-term method detection level; MDL, method detection limit; MRL, minimum reporting level; $\mathrm{mg} / \mathrm{L}$, milligrams per liter; na, not available; D, detected in groundwater samples (table 10)]

\begin{tabular}{|c|c|c|c|c|c|c|}
\hline Constituent & $\begin{array}{c}\text { USGS } \\
\text { parameter } \\
\text { code }\end{array}$ & $\begin{array}{c}\text { CAS } \\
\text { registry } \\
\text { number }^{\circledR}\end{array}$ & $\begin{array}{c}\text { LT-MDL, } \\
\text { MDL, or MRL } \\
(\mathrm{mg} / \mathrm{L})^{1}\end{array}$ & $\begin{array}{c}\text { Benchmark } \\
\text { type }\end{array}$ & $\begin{array}{c}\text { Benchmark } \\
\text { level } \\
\text { (mg/L) }\end{array}$ & Detection \\
\hline Bromide & 71870 & $24959-67-9$ & 0.010 & na & na & $\mathrm{D}$ \\
\hline Calcium & 00915 & $7440-70-2$ & 0.022 & na & na & $\mathrm{D}$ \\
\hline Chloride & 00940 & $16887-00-6$ & 0.06 & SMCL-CA & ${ }^{2} 250(500)$ & $\mathrm{D}$ \\
\hline Fluoride & 00950 & $16984-48-8$ & 0.04 & MCL-CA & 2 & $\mathrm{D}$ \\
\hline Magnesium & 00925 & $7439-95-4$ & $0.008,0.011$ & na & na & $\mathrm{D}$ \\
\hline Potassium & 00935 & $7440-09-7$ & $0.022,0.03$ & na & na & $\mathrm{D}$ \\
\hline Silica $\left(\right.$ as $\left.\mathrm{SiO}_{2}\right)$ & 00955 & $7631-86-9$ & $0.029,0.018$ & na & na & $\mathrm{D}$ \\
\hline Sodium & 00930 & $7440-23-5$ & 0.06 & na & na & $\mathrm{D}$ \\
\hline Laboratory alkalinity (as $\mathrm{CaCO}_{3}$ ) & 29801 & na & $4.0,4.6$ & na & na & $\mathrm{D}^{3}$ \\
\hline
\end{tabular}

${ }^{1}$ For constituents with two reporting levels (LT-MDL, MDL, or MRL) listed, the first value was in use before October 1, 2011, and the second value was in use starting October 1, 2011. The highest reporting level is used for this report.

${ }^{2}$ The recommended SMCL-CA benchmarks for chloride, sulfate, and TDS are listed with the upper SMCL-CA benchmarks in parentheses.

${ }^{3}$ Laboratory alkalinity results are presented in table 4. 
Table 3G. Arsenic, chromium, and iron species, reporting information, and comparative benchmarks for the U.S. Geological Survey (USGS) Trace Metal Laboratory, Boulder, Colorado, analyses.

[The five-digit USGS parameter code is used to uniquely identify a specific constituent or property. Reporting levels, benchmark types, and benchmark levels as of July 6, 2012. Benchmark type: maximum contaminant levels are listed as MCL-US when the MCL-US and MCL-CA are identical, and as MCL-CA when the MCL-CA is lower than the MCL-US or no MCL-US exists. Secondary maximum contaminant level benchmarks are listed as SMCL-CA when the SMCL-CA exists, and as the SMCL-US when no SMCL-CA exists. MCL-CA, California Department of Public Health (CDPH) maximum contaminant level; SMCL-CA, CDPH secondary maximum contaminant level. Other abbreviations: CAS, Chemical Abstracts Service; MDL, method detection limit; na, not available; $\mu \mathrm{g} / \mathrm{L}$, micrograms per liter; $\mathrm{D}$, detected in groundwater samples (table 11)]

\begin{tabular}{|c|c|c|c|c|c|c|}
\hline Constituent & $\begin{array}{c}\text { USGS } \\
\text { parameter code }\end{array}$ & $\begin{array}{c}\text { CAS registry } \\
\text { number }^{\circledR}\end{array}$ & $\begin{array}{l}\text { MDL } \\
(\mu \mathrm{g} / \mathrm{L})\end{array}$ & $\begin{array}{c}\text { Benchmark } \\
\text { type }\end{array}$ & $\begin{array}{c}\text { Benchmark level } \\
\text { ( } \mu \mathrm{g} / \mathrm{L})\end{array}$ & Detection \\
\hline Arsenic (total) & 99033 & $7440-38-2$ & 0.2 & MCL-US & 10 & $\mathrm{D}$ \\
\hline Chromium (total) & 01030 & $7440-47-3$ & 1 & MCL-CA & 50 & $\mathrm{D}$ \\
\hline Chromium (VI) & 01032 & $18540-29-9$ & 1 & na & na & $\mathrm{D}$ \\
\hline
\end{tabular}


Table 3H. Isotopic and radioactive constituents, reporting information, and comparative benchmarks for laboratory analyses.

[The five-digit U.S. Geological Survey (USGS) parameter code is used to uniquely identify a specific constituent or property. Stable isotope ratios are reported in the standard delta notation $(\delta)$, the ratio of a heavier isotope to more common lighter isotope of that element, relative to a standard reference material. Reporting levels, benchmark types, and benchmark levels as of July 6, 2012. Benchmark type: Maximum contaminant levels are listed as MCL-US when the MCL-US and MCL-CA are identical, and as MCL-CA when the MCL-CA is lower than the MCL-US or no MCL-US exists. MCL-US, U.S. Environmental Protection Agency maximum contaminant level; MCL-CA, California Department of Public Health maximum contaminant level. Other abbreviations: CAS, Chemical

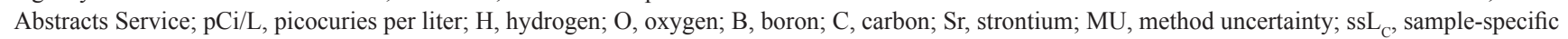
critical level; CSU, 1-sigma combined standard uncertainty; na, not available; D, detected in groundwater samples (tables 12 and $14 \mathrm{~A}-\mathrm{C}$ )]

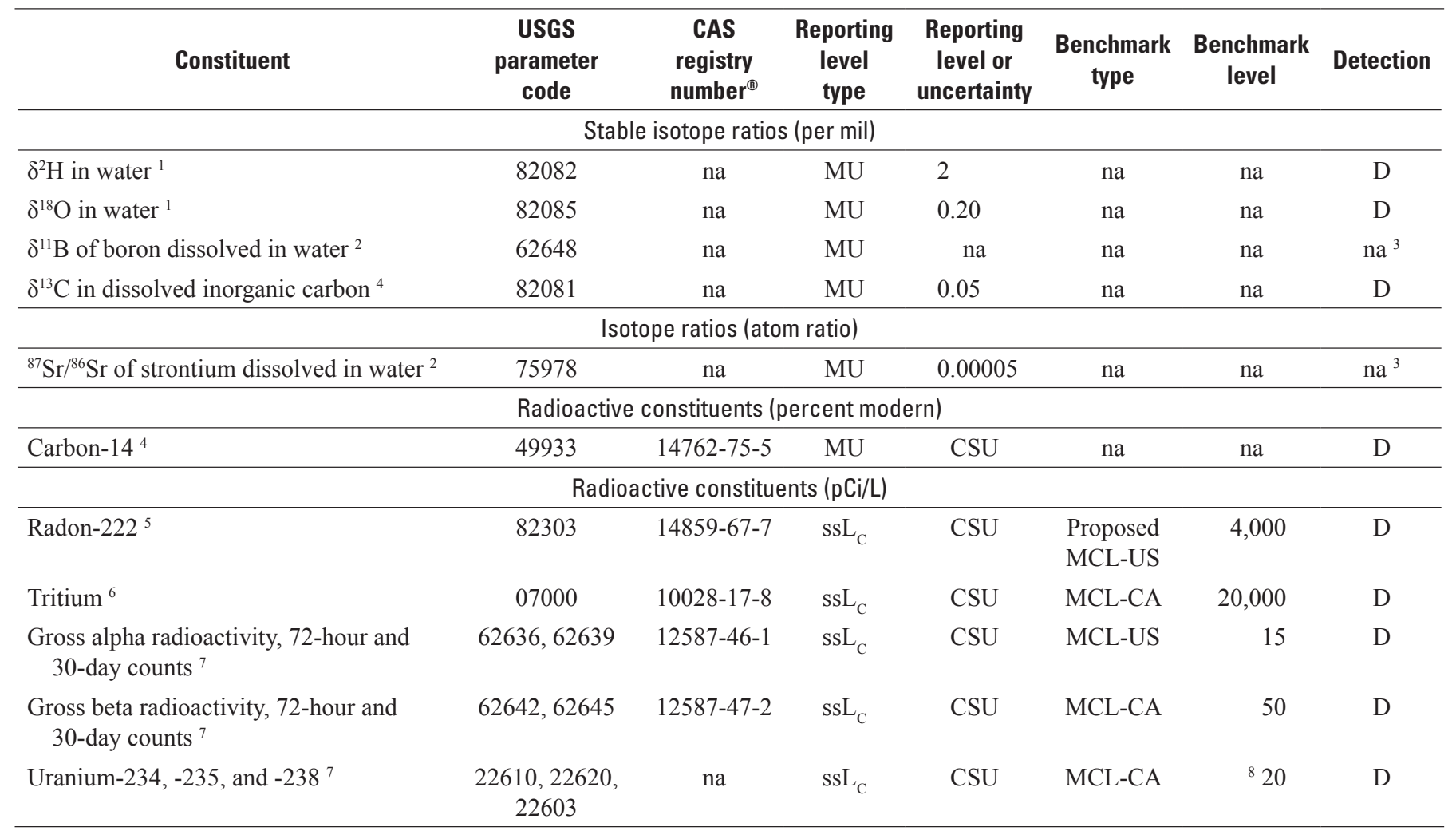

\footnotetext{
${ }^{1}$ USGS Stable Isotope Laboratory, Reston, Virginia (USGSSIVA).

${ }^{2}$ USGS NRP Metals Isotope Research Laboratory, Menlo Park, California (USGSMICA).

${ }^{3}$ Stable isotopes of dissolved boron and strontium in water data will be included in a subsequent report.

${ }^{4}$ Woods Hole Oceanographic Institution, National Ocean Sciences Accelerator Mass Spectrometry Facility (NOSAMS), Woods Hole, Massachusetts (MA-WHAMS).

${ }^{5}$ USGS National Water Quality Laboratory (USGSNWQL).

${ }^{6}$ USGS Stable Isotope and Tritium Laboratory, Menlo Park, California (USGSH3CA).

${ }^{7}$ Eberline Analytical Services, Richmond, California (CA-EBERL).

${ }^{8}$ The MCL-CA benchmark for uranium is the sum of uranium-234, -235, and -238 .
} 
Table 31. Dissolved noble gases and tritium, reporting information, and comparative benchmarks for the Lawrence Livermore National Laboratory (LLNL) analyses.

[Results for noble gas and tritium analyses by the LLNL are stored in a database maintained by Groundwater Ambient Monitoring and Assessment (GAMA) Priority Basin Project project staff. Benchmark types and benchmark values as of July 6, 2012. Benchmark type: Maximum contaminant level benchmarks are listed as MCL-US when the MCL-US and MCL-CA are identical, and as MCL-CA when the MCL-CA is lower than the MCL-US or no MCL-US exists. MCL-CA, California Department of Public Health maximum contaminant level. Other abbreviations: CAS, Chemical Abstracts Service; na, not available; $\mathrm{cm}^{3} \mathrm{STP} / \mathrm{g}$, cubic centimeters of gas at standard temperature and pressure per gram of water; D, detected in groundwater samples (table 13)]

\begin{tabular}{|c|c|c|c|c|c|c|}
\hline Constituent & $\begin{array}{c}\text { CAS } \\
\text { registry } \\
\text { number }^{\circledR}\end{array}$ & $\begin{array}{l}\text { Reporting } \\
\text { units }\end{array}$ & $\begin{array}{l}\text { Method } \\
\text { uncertainty } \\
\text { (percent) }\end{array}$ & $\begin{array}{c}\text { Benchmark } \\
\text { type }\end{array}$ & $\begin{array}{c}\text { Benchmark } \\
\text { level }\end{array}$ & Detection \\
\hline Argon & $7440-37-1$ & $\mathrm{~cm}^{3} \mathrm{STP} / \mathrm{g}$ & 2 & na & na & $\mathrm{D}$ \\
\hline Helium-3/Helium-4 & na / 7440-59-7 & atom ratio & ${ }^{1} 1.5$ & na & na & $\mathrm{D}$ \\
\hline Helium-4 & $7440-59-7$ & $\mathrm{~cm}^{3} \mathrm{STP} / \mathrm{g}$ & 2 & na & na & $\mathrm{D}$ \\
\hline Krypton & 7439-90-9 & $\mathrm{cm}^{3} \mathrm{STP} / \mathrm{g}$ & 3 & na & na & $\mathrm{D}$ \\
\hline Tritium & $10028-17-8$ & $\mathrm{pCi} / \mathrm{L}$ & 1 & MCL-CA & 20,000 & $\mathrm{na}^{2}$ \\
\hline Xenon & $7440-63-3$ & $\mathrm{~cm}^{3} \mathrm{STP} / \mathrm{g}$ & 1 & na & na & $\mathrm{D}$ \\
\hline
\end{tabular}

${ }^{1}$ Helium-3/Helium-4 results for the Hard Rock study unit had uncertainties ranging from 1 to 9 percent of the reported concentrations (the median was 1.5 percent).

${ }^{2}$ Results for tritium analyzed at the LLNL were not available at the time of this report. 
Table 4. Water-quality indicators in samples collected for the Santa Cruz, San Gabriel, and Peninsular Ranges Hard Rock Aquifers (Hard Rock) study unit, March 2011 through March 2012, California GAMA Priority Basin Project.

[The five-digit U.S. Geological Survey (USGS) parameter code below the constituent name is used to uniquely identify a specific constituent or property. Field alkalinities were measured at only 15 percent of the Hard Rock sites. GAMA well identification number: HR-SC, Santa Cruz study area grid well; HR-SG, San Gabriel study area grid well; HR-SGU, San Gabriel study area understanding well; HR-PR, Peninsular Ranges study area grid well; HR-PRFP, Peninsular Ranges study area flow-path well; HR-PRU, Peninsular Ranges study area understanding well. Reporting level, benchmark type, and benchmark level as of July 6, 2012. Benchmark type: Maximum contamination level benchmarks are listed as SMCL-CA when the SMCL-CA exists, and as the SMCL-US when no SMCL-CA exists. SMCL-US,

U.S. Environmental Protection Agency secondary maximum contaminant level; SMCL-CA, California Department of Public Health secondary maximum contaminant level. Other abbreviations: ${ }^{\circ} \mathrm{C}$, degrees Celsius; mg/L, milligrams per liter; na, not available; $\mu \mathrm{S} / \mathrm{cm}$, microsiemens per centimeter; <, less than; >, greater than; $\mathrm{CaCO}_{3}$, calcium carbonate; $\mathrm{HCO}_{3}^{-}$, bicarbonate; $\mathrm{CO}_{3}{ }^{2-}$, carbonate; *, value greater than benchmark level or outside range; **, value greater than upper benchmark level; —, not detected]

\begin{tabular}{|c|c|c|c|c|c|c|c|c|c|c|c|c|}
\hline $\begin{array}{c}\text { GAMA well } \\
\text { identification } \\
\text { number }\end{array}$ & $\begin{array}{l}\text { Dissolved } \\
\text { oxygen, } \\
\text { field } \\
(\mathrm{mg} / \mathrm{L}) \\
(00300)\end{array}$ & $\begin{array}{c}\text { Water } \\
\text { temperature, } \\
\text { field } \\
\left({ }^{\circ} \mathrm{C}\right) \\
(00010)\end{array}$ & $\begin{array}{c}\text { pH, field } \\
\text { (standard } \\
\text { units) } \\
(00400)\end{array}$ & $\begin{array}{c}\text { pH, } \\
\text { laboratory } \\
\text { (standard } \\
\text { units) } \\
(00403)\end{array}$ & $\begin{array}{c}\text { Specific } \\
\text { conductance, } \\
\text { field } \\
(\mu \mathrm{S} / \mathrm{cm} \text { at } \\
\left.25^{\circ} \mathrm{C}\right) \\
(00095)\end{array}$ & $\begin{array}{c}\text { Specific } \\
\text { conductance, } \\
\text { laboratory } \\
(\mu \mathrm{S} / \mathrm{cm} \text { at } \\
\left.25^{\circ} \mathrm{C}\right) \\
(90095)\end{array}$ & $\begin{array}{c}\text { Alkalinity, } \\
\text { laboratory } \\
\text { (mg/L as } \\
\left.\mathrm{CaCO}_{3}\right) \\
(29801)\end{array}$ & $\begin{array}{c}\text { Bicarbonate, } \\
\text { laboratory } \\
\text { (mg/L as } \\
\left.\mathrm{HCO}_{3}^{-}\right)^{1}\end{array}$ & $\begin{array}{c}\text { Carbonate, } \\
\text { laboratory } \\
(\mathrm{mg} / \mathrm{L} \text { as } \\
\left.\mathrm{CO}_{3}{ }^{2-}\right)^{1}\end{array}$ & $\begin{array}{c}\text { Alkalinity, } \\
\text { field } \\
\text { (mg/L as } \\
\left.\mathrm{CaCO}_{3}\right) \\
(29802)\end{array}$ & $\begin{array}{c}\text { Bicarbonate, } \\
\text { field } \\
\text { (mg/L as } \\
\left.\mathrm{HCO}_{3}^{-}\right) \\
(63786)\end{array}$ & $\begin{array}{c}\text { Carbonate, } \\
\text { field } \\
\text { (mg/L as } \\
\left.\mathrm{CO}_{3}^{2-}\right) \\
(63788)\end{array}$ \\
\hline Benchmark type & na & na & SMCL-US & SMCL-US & SMCL-CA & SMCL-CA & na & na & na & na & na & na \\
\hline Benchmark level & na & na & $<6.5$ or $>8.5$ & $<6.5$ or $>8.5$ & ${ }^{2} 900(1,600)$ & ${ }^{2} 900(1,600)$ & na & na & na & na & na & na \\
\hline $\begin{array}{l}\text { [Reporting level } \\
\text { or range] }\end{array}$ & {$[0.2]$} & {$[0.0-38.5]$} & {$[0-14]$} & {$[0-14]$} & [5] & [5] & [4.6] & [1] & {$[0.1]$} & [1] & [1] & {$[0.1]$} \\
\hline
\end{tabular}

\section{Santa Cruz study area (25 wells sampled)}

\begin{tabular}{|c|c|c|c|c|c|c|c|c|c|c|c|c|}
\hline HR-SC-01 & 5.2 & 18.5 & 8.1 & 8.1 & 289 & 282 & 69.5 & 83.6 & 0.5 & na & na & na \\
\hline HR-SC-02 & 7.5 & 14.0 & 6.9 & 7.1 & 394 & 429 & 95 & 116 & - & na & na & na \\
\hline HR-SC-03 & 0.2 & 15.0 & 7.2 & 7.2 & 704 & 695 & 204 & 248 & 0.2 & 209 & 255 & - \\
\hline HR-SC-04 & 1.3 & 13.5 & 6.9 & 7.2 & 889 & 872 & 227 & 277 & 0.2 & na & na & na \\
\hline HR-SC-05 & - & 14.0 & 7.1 & 7.4 & 264 & 263 & 112 & 137 & 0.2 & na & na & na \\
\hline HR-SC-06 & 5.5 & 14.0 & 6.5 & 6.9 & 411 & 412 & 176 & 214 & - & na & na & na \\
\hline HR-SC-07 & - & 19.5 & 7.1 & 7.3 & $* * 1,690$ & $* * 1,700$ & 322 & 392 & 0.4 & na & na & na \\
\hline HR-SC-08 & 0.6 & 16.0 & 6.5 & 6.8 & 412 & 419 & 166 & 203 & - & na & na & na \\
\hline HR-SC-09 & - & 19.5 & 8.2 & 8.5 & $* 1,060$ & * 991 & 521 & 617 & 9.0 & 453 & 533 & 9.2 \\
\hline HR-SC-10 & 1.0 & 13.0 & 6.5 & 7.0 & 291 & 291 & 132 & 161 & - & na & na & na \\
\hline HR-SC-11 & 6.0 & 15.5 & 6.9 & 7.2 & 710 & 717 & 324 & 394 & 0.3 & na & na & na \\
\hline HR-SC-12 & 0.2 & 17.5 & 7.2 & 7.4 & 868 & 873 & 457 & 556 & 0.7 & na & na & na \\
\hline HR-SC-13 & 8.2 & 14.5 & 6.9 & 7.4 & 264 & 269 & 120 & 146 & 0.2 & na & na & na \\
\hline HR-SC-14 & 0.3 & 14.5 & $* 6.1$ & 6.5 & 527 & 540 & 160 & 196 & - & na & na & na \\
\hline HR-SC-15 & - & 17.0 & $* 8.6$ & $* 8.8$ & 504 & 510 & 220 & 253 & 7.0 & na & na & na \\
\hline
\end{tabular}


Table 4. Water-quality indicators in samples collected for the Santa Cruz, San Gabriel, and Peninsular Ranges Hard Rock Aquifers (Hard Rock) study unit, March 2011 through March 2012, California GAMA Priority Basin Project.-Continued

[The five-digit U.S. Geological Survey (USGS) parameter code below the constituent name is used to uniquely identify a specific constituent or property. Field alkalinities were measured at only 15 percent of the Hard Rock sites. GAMA well identification number: HR-SC, Santa Cruz study area grid well; HR-SG, San Gabriel study area grid well; HR-SGU, San Gabriel study area understanding well; HR-PR, Peninsular Ranges study area grid well; HR-PRFP, Peninsular Ranges study area flow-path well; HR-PRU, Peninsular Ranges study area understanding well. Reporting level, benchmark type, and benchmark level as of July 6, 2012. Benchmark type: Maximum contamination level benchmarks are listed as SMCL-CA when the SMCL-CA exists, and as the SMCL-US when no SMCL-CA exists. SMCL-US,

U.S. Environmental Protection Agency secondary maximum contaminant level; SMCL-CA, California Department of Public Health secondary maximum contaminant level. Other abbreviations: ${ }^{\circ} \mathrm{C}$, degrees Celsius; $\mathrm{mg} / \mathrm{L}$, milligrams per liter; na, not available; $\mu \mathrm{S} / \mathrm{cm}$, microsiemens per centimeter; $<$, less than; $>$, greater than; $\mathrm{CaCO}_{3}$, calcium carbonate; $\mathrm{HCO}_{3}{ }^{-}$, bicarbonate; $\mathrm{CO}_{3}{ }^{2-}$, carbonate; *, value greater than benchmark level or outside range; **, value greater than upper benchmark level; - , not detected]

\begin{tabular}{|c|c|c|c|c|c|c|c|c|c|c|c|c|}
\hline $\begin{array}{c}\text { GAMA well } \\
\text { identification } \\
\text { number }\end{array}$ & $\begin{array}{l}\text { Dissolved } \\
\text { oxygen, } \\
\text { field } \\
(\text { mg/L) } \\
(00300)\end{array}$ & $\begin{array}{c}\text { Water } \\
\text { temperature, } \\
\text { field } \\
\left({ }^{\circ} \mathrm{C}\right) \\
(00010)\end{array}$ & $\begin{array}{c}\text { pH, field } \\
\text { (standard } \\
\text { units) } \\
(00400)\end{array}$ & $\begin{array}{c}\text { pH, } \\
\text { laboratory } \\
\text { (standard } \\
\text { units) } \\
\text { (00403) }\end{array}$ & $\begin{array}{c}\text { Specific } \\
\text { conductance, } \\
\text { field } \\
(\mu \mathrm{S} / \mathrm{cm} \text { at } \\
\left.25^{\circ} \mathrm{C}\right) \\
(00095)\end{array}$ & $\begin{array}{l}\text { Specific } \\
\text { conductance, } \\
\text { laboratory } \\
(\mu \mathrm{S} / \mathrm{cm} \text { at } \\
\left.25^{\circ} \mathrm{C}\right) \\
(90095)\end{array}$ & $\begin{array}{c}\text { Alkalinity, } \\
\text { laboratory } \\
\text { (mg/L as } \\
\left.\mathrm{CaCO}_{3}\right) \\
(29801)\end{array}$ & $\begin{array}{c}\text { Bicarbonate, } \\
\text { laboratory } \\
(\mathrm{mg} / \mathrm{L} \text { as } \\
\left.\mathrm{HCO}_{3}^{-}\right)^{1}\end{array}$ & $\begin{array}{c}\text { Carbonate, } \\
\text { laboratory } \\
\text { (mg/L as } \\
\left.\mathrm{CO}_{3}{ }^{2-}\right)^{1}\end{array}$ & $\begin{array}{c}\text { Alkalinity, } \\
\text { field } \\
\text { (mg/L as } \\
\left.\mathrm{CaCO}_{3}\right) \\
(29802)\end{array}$ & $\begin{array}{c}\text { Bicarbonate, } \\
\text { field } \\
\text { (mg/L as } \\
\mathrm{HCO}_{3}^{-} \text {) } \\
(63786)\end{array}$ & $\begin{array}{c}\text { Carbonate, } \\
\text { field } \\
(\mathrm{mg} / \mathrm{L} \text { as } \\
\left.\mathrm{CO}_{3}^{2-}\right) \\
(63788)\end{array}$ \\
\hline Benchmark type & na & na & SMCL-US & SMCL-US & SMCL-CA & SMCL-CA & na & na & na & na & na & na \\
\hline Benchmark level & na & na & $<6.5$ or $>8.5$ & $<6.5$ or $>8.5$ & ${ }^{2} 900(1,600)$ & ${ }^{2} 900(1,600)$ & na & na & na & na & na & na \\
\hline $\begin{array}{l}\text { [Reporting level } \\
\text { or range] }\end{array}$ & [0.2] & {$[0.0-38.5]$} & [0-14] & {$[0-14]$} & [5] & [5] & [4.6] & [1] & {$[0.1]$} & [1] & [1] & {$[0.1]$} \\
\hline \multicolumn{13}{|c|}{ Santa Cruz study area ( 25 wells sampled)—Continued } \\
\hline HR-SC-16 & 2.2 & 13.0 & 7.7 & 8.1 & 252 & 250 & 119 & 143 & 0.8 & na & na & na \\
\hline HR-SC-17 & 5.9 & 16.0 & $* 5.7$ & $* 36.1$ & 89 & 387 & 25.8 & 31.5 & - & 22.2 & 27.1 & - \\
\hline HR-SC-18 & 0.6 & 18.0 & 7.0 & 7.4 & 195 & 192 & 54.7 & 66.5 & - & na & na & na \\
\hline HR-SC-19 & - & 17.0 & 7.3 & 7.6 & 782 & 790 & 334 & 406 & 0.7 & na & na & na \\
\hline HR-SC-20 & 5.8 & 16.0 & 6.7 & 7.0 & $* 1,480$ & $* 1,530$ & 319 & 388 & 0.2 & na & na & na \\
\hline HR-SC-21 & 0.2 & 19.0 & 7.2 & 7.4 & $* 1,120$ & $* 1,170$ & 265 & 323 & 0.4 & na & na & na \\
\hline HR-SC-22 & - & 15.0 & 6.6 & 6.9 & 839 & 862 & 369 & 450 & 0.2 & 364 & 444 & 0.1 \\
\hline HR-SC-23 & 0.2 & 18.0 & 7.1 & 7.4 & 792 & 791 & 349 & 425 & 0.4 & na & na & na \\
\hline HR-SC-24 & 0.8 & 17.5 & 7.0 & 7.4 & $* 1,470$ & $* 1,480$ & 544 & 662 & 0.7 & na & na & na \\
\hline HR-SC-25 & 9.8 & 15.0 & $* 6.4$ & 6.8 & 134 & 135 & 41.7 & 50.8 & - & na & na & na \\
\hline \multicolumn{13}{|c|}{ San Gabriel study area (37 wells sampled) } \\
\hline HR-SG-01 & 3.4 & 14.0 & 7.4 & 7.6 & 739 & 736 & 256 & 311 & 0.5 & na & na & na \\
\hline HR-SG-02 & 1.4 & 15.0 & 7.2 & 7.5 & 654 & 664 & 273 & 332 & 0.5 & na & na & na \\
\hline HR-SG-03 & 1.5 & 14.5 & 7.3 & 7.6 & 630 & 622 & 265 & 322 & 0.6 & na & na & na \\
\hline HR-SG-04 & 6.2 & 20.0 & 7.2 & 7.5 & $* 1,080$ & $* 1,050$ & 330 & 401 & 0.6 & na & na & na \\
\hline HR-SG-05 & 6.9 & 16.0 & 7.3 & 7.5 & 663 & 660 & 291 & 354 & 0.5 & na & na & na \\
\hline
\end{tabular}


Table 4. Water-quality indicators in samples collected for the Santa Cruz, San Gabriel, and Peninsular Ranges Hard Rock Aquifers (Hard Rock) study unit, March 2011 through March 2012, California GAMA Priority Basin Project.-Continued

[The five-digit U.S. Geological Survey (USGS) parameter code below the constituent name is used to uniquely identify a specific constituent or property. Field alkalinities were measured at only 15 percent of the Hard Rock sites. GAMA well identification number: HR-SC, Santa Cruz study area grid well; HR-SG, San Gabriel study area grid well; HR-SGU, San Gabriel study area understanding well; HR-PR, Peninsular Ranges study area grid well; HR-PRFP, Peninsular Ranges study area flow-path well; HR-PRU, Peninsular Ranges study area understanding well. Reporting level, benchmark type, and benchmark level as of July 6, 2012. Benchmark type: Maximum contamination level benchmarks are listed as SMCL-CA when the SMCL-CA exists, and as the SMCL-US when no SMCL-CA exists. SMCL-US,

U.S. Environmental Protection Agency secondary maximum contaminant level; SMCL-CA, California Department of Public Health secondary maximum contaminant level. Other abbreviations: ${ }^{\circ} \mathrm{C}$, degree Celsius; mg/L, milligrams per liter; na, not available; $\mu \mathrm{S} / \mathrm{cm}$, microsiemens per centimeter; <, less than; >, greater than; $\mathrm{CaCO}_{3}$, calcium carbonate; $\mathrm{HCO}_{3}^{-}$, bicarbonate; $\mathrm{CO}_{3}{ }^{2-}$, carbonate; *, value greater than benchmark level or outside range; **, value greater than upper benchmark level; 一, not detected]

\begin{tabular}{|c|c|c|c|c|c|c|c|c|c|c|c|c|}
\hline $\begin{array}{c}\text { GAMA well } \\
\text { identification } \\
\text { number }\end{array}$ & $\begin{array}{c}\text { Dissolved } \\
\text { oxygen, } \\
\text { field } \\
(\mathrm{mg} / \mathrm{L}) \\
(00300)\end{array}$ & $\begin{array}{c}\text { Water } \\
\text { temperature, } \\
\text { field } \\
\left({ }^{\circ} \mathrm{C}\right) \\
(00010)\end{array}$ & $\begin{array}{c}\text { pH, field } \\
\text { (standard } \\
\text { units) } \\
(00400)\end{array}$ & $\begin{array}{c}\text { pH, } \\
\text { laboratory } \\
\text { (standard } \\
\text { units) } \\
\text { (00403) }\end{array}$ & $\begin{array}{c}\text { Specific } \\
\text { conductance, } \\
\text { field } \\
(\mu \mathrm{S} / \mathrm{cm} \text { at } \\
\left.25^{\circ} \mathrm{C}\right) \\
(00095)\end{array}$ & $\begin{array}{c}\text { Specific } \\
\text { conductance, } \\
\text { laboratory } \\
(\mu \mathrm{S} / \mathrm{cm} \text { at } \\
\left.25^{\circ} \mathrm{C}\right) \\
(90095)\end{array}$ & $\begin{array}{c}\text { Alkalinity, } \\
\text { laboratory } \\
\text { (mg/L as } \\
\text { CaCO }) \\
(29801)\end{array}$ & $\begin{array}{c}\text { Bicarbonate, } \\
\text { laboratory } \\
(\mathrm{mg} / \mathrm{L} \text { as } \\
\left.\mathrm{HCO}_{3}^{-}\right)^{1}\end{array}$ & $\begin{array}{c}\text { Carbonate, } \\
\text { laboratory } \\
\text { (mg/L as } \\
\left.\mathrm{CO}_{3}{ }^{2-}\right)^{1}\end{array}$ & $\begin{array}{l}\text { Alkalinity, } \\
\text { field } \\
\text { (mg/L as } \\
\left.\mathrm{CaCO}_{3}\right) \\
(29802)\end{array}$ & $\begin{array}{c}\text { Bicarbonate, } \\
\text { field } \\
\text { (mg/L as } \\
\left.\mathrm{HCO}_{3}^{-}\right) \\
(63786)\end{array}$ & $\begin{array}{c}\text { Carbonate, } \\
\text { field } \\
\text { (mg/L as } \\
\left.\mathrm{CO}_{3}^{2-}\right) \\
(63788)\end{array}$ \\
\hline Benchmark type & na & na & SMCL-US & SMCL-US & SMCL-CA & SMCL-CA & na & na & na & na & na & na \\
\hline Benchmark level & na & na & $<6.5$ or $>8.5$ & $<6.5$ or $>8.5$ & ${ }^{2} 900(1,600)$ & ${ }^{2} 900(1,600)$ & na & na & na & na & na & na \\
\hline $\begin{array}{l}\text { [Reporting level } \\
\text { or range] }\end{array}$ & {$[0.2]$} & {$[0.0-38.5]$} & {$[0-14]$} & {$[0-14]$} & {$[5]$} & {$[5]$} & {$[4.6]$} & [1] & {$[0.1]$} & [1] & [1] & {$[0.1]$} \\
\hline \multicolumn{13}{|c|}{ San Gabriel study area (37 wells sampled)_Continued } \\
\hline HR-SG-06 & 6.2 & 17.0 & 7.3 & 7.6 & 338 & 332 & 140 & 170 & 0.3 & na & na & na \\
\hline HR-SG-07 & 0.2 & 15.5 & 7.6 & 7.8 & 349 & 344 & 165 & 200 & 0.6 & na & na & na \\
\hline HR-SG-08 & - & 16.0 & 7.4 & 7.5 & 830 & 822 & 333 & 405 & 0.6 & na & na & na \\
\hline HR-SG-09 & 0.5 & 22.5 & $* 8.9$ & $* 8.9$ & $* 1,220$ & $* 1,210$ & 281 & 318 & 11.6 & 251 & 278 & 13.4 \\
\hline HR-SG-10 & 7.3 & 17.0 & 6.9 & 7.2 & 625 & 625 & 243 & 296 & 0.2 & na & na & na \\
\hline HR-SG-11 & 6.5 & 20.0 & * 8.8 & $* 8.8$ & 337 & 338 & 126 & 145 & 4.2 & na & na & na \\
\hline HR-SG-12 & 5.1 & 10.0 & 6.7 & 7.4 & 349 & 358 & 190 & 231 & 0.3 & na & na & na \\
\hline HR-SG-13 & 2.8 & 11.0 & 6.9 & 7.3 & 546 & 547 & 258 & 314 & 0.3 & 265 & 323 & 0.3 \\
\hline HR-SG-14 & 0.4 & 12.0 & 6.7 & 7.2 & 615 & 633 & 258 & 314 & 0.2 & na & na & na \\
\hline HR-SG-15 & 3.2 & 19.0 & 6.8 & 7.1 & 303 & 289 & 142 & 173 & 0.1 & na & na & na \\
\hline HR-SG-16 & 1.9 & 18.5 & 7.3 & 7.5 & 418 & 420 & 181 & 220 & 0.3 & na & na & na \\
\hline HR-SG-17 & 2.3 & 10.5 & 6.7 & 7.4 & 305 & 313 & 168 & 204 & 0.2 & na & na & na \\
\hline HR-SG-18 & 0.8 & 14.5 & 7.0 & 7.4 & 540 & 539 & 252 & 307 & 0.4 & na & na & na \\
\hline HR-SG-19 & 2.1 & 13.0 & 7.0 & 7.3 & 559 & 559 & 259 & 315 & 0.3 & na & na & na \\
\hline HR-SG-20 & 2.8 & 17.0 & 7.3 & 7.3 & 686 & 702 & 304 & 370 & 0.3 & 301 & 366 & 0.5 \\
\hline
\end{tabular}


Table 4. Water-quality indicators in samples collected for the Santa Cruz, San Gabriel, and Peninsular Ranges Hard Rock Aquifers (Hard Rock) study unit, March 2011 through

March 2012, California GAMA Priority Basin Project.-Continued

[The five-digit U.S. Geological Survey (USGS) parameter code below the constituent name is used to uniquely identify a specific constituent or property. Field alkalinities were measured at only 15 percent of the Hard Rock sites. GAMA well identification number: HR-SC, Santa Cruz study area grid well; HR-SG, San Gabriel study area grid well; HR-SGU, San Gabriel study area understanding well; HR-PR, Peninsular Ranges study area grid well; HR-PRFP, Peninsular Ranges study area flow-path well; HR-PRU, Peninsular Ranges study area understanding well. Reporting level, benchmark type, and benchmark level as of July 6, 2012. Benchmark type: Maximum contamination level benchmarks are listed as SMCL-CA when the SMCL-CA exists, and as the SMCL-US when no SMCL-CA exists. SMCL-US,

U.S. Environmental Protection Agency secondary maximum contaminant level; SMCL-CA, California Department of Public Health secondary maximum contaminant level. Other abbreviations: ${ }^{\circ} \mathrm{C}$, degrees Celsius; mg/L, milligrams per liter; na, not available; $\mu \mathrm{S} / \mathrm{cm}$, microsiemens per centimeter; $<$, less than; >, greater than; $\mathrm{CaCO}_{3}$, calcium carbonate; $\mathrm{HCO}_{3}^{-}$, bicarbonate; $\mathrm{CO}_{3}{ }^{2-}$, carbonate; *, value greater than benchmark level or outside range; ${ }^{* *}$, value greater than upper benchmark level; —, not detected]

\begin{tabular}{|c|c|c|c|c|c|c|c|c|c|c|c|c|}
\hline $\begin{array}{c}\text { GAMA well } \\
\text { identification } \\
\text { number }\end{array}$ & $\begin{array}{l}\text { Dissolved } \\
\text { oxygen, } \\
\text { field } \\
(\mathrm{mg} / \mathrm{L}) \\
(00300)\end{array}$ & $\begin{array}{c}\text { Water } \\
\text { temperature, } \\
\text { field } \\
\left({ }^{\circ} \mathrm{C}\right) \\
(00010)\end{array}$ & $\begin{array}{c}\text { pH, field } \\
\text { (standard } \\
\text { units) } \\
(00400)\end{array}$ & $\begin{array}{c}\text { pH, } \\
\text { laboratory } \\
\text { (standard } \\
\text { units) } \\
(00403)\end{array}$ & $\begin{array}{c}\text { Specific } \\
\text { conductance, } \\
\text { field } \\
(\mu \mathrm{S} / \mathrm{cm} \text { at } \\
\left.25^{\circ} \mathrm{C}\right) \\
(00095)\end{array}$ & $\begin{array}{c}\text { Specific } \\
\text { conductance, } \\
\text { laboratory } \\
(\mu \mathrm{S} / \mathrm{cm} \text { at } \\
\left.25^{\circ} \mathrm{C}\right) \\
(90095)\end{array}$ & $\begin{array}{c}\text { Alkalinity, } \\
\text { laboratory } \\
\text { (mg/L as } \\
\left.\mathrm{CaCO}_{3}\right) \\
(29801)\end{array}$ & $\begin{array}{c}\text { Bicarbonate, } \\
\text { laboratory } \\
(\mathrm{mg} / \mathrm{L} \text { as } \\
\left.\mathrm{HCO}_{3}^{-}\right)^{1}\end{array}$ & $\begin{array}{c}\text { Carbonate, } \\
\text { laboratory } \\
\text { (mg/L as } \\
\left.\mathrm{CO}_{3}{ }^{2-}\right)^{1}\end{array}$ & $\begin{array}{l}\text { Alkalinity, } \\
\text { field } \\
\text { (mg/L as } \\
\mathrm{CaCO}_{3} \text { ) } \\
(29802)\end{array}$ & $\begin{array}{c}\text { Bicarbonate, } \\
\text { field } \\
\text { (mg/L as } \\
\mathrm{HCO}_{3}^{-} \text {) } \\
(63786)\end{array}$ & $\begin{array}{c}\text { Carbonate, } \\
\text { field } \\
\text { (mg/L as } \\
\left.\mathrm{CO}_{3}^{2-}\right) \\
(63788)\end{array}$ \\
\hline Benchmark type & na & na & SMCL-US & SMCL-US & SMCL-CA & SMCL-CA & na & na & na & na & na & na \\
\hline Benchmark level & na & na & $<6.5$ or $>8.5$ & $<6.5$ or $>8.5$ & ${ }^{2} 900(1,600)$ & ${ }^{2} 900(1,600)$ & na & na & na & na & na & na \\
\hline $\begin{array}{l}\text { [Reporting level } \\
\text { or range] }\end{array}$ & {$[0.2]$} & {$[0.0-38.5]$} & [0-14] & {$[0-14]$} & [5] & [5] & [4.6] & [1] & {$[0.1]$} & [1] & [1] & {$[0.1]$} \\
\hline \multicolumn{13}{|c|}{ San Gabriel study area (37 wells sampled)_Continued } \\
\hline HR-SG-21 & 6.4 & 11.5 & 6.8 & 7.2 & 454 & 464 & 240 & 292 & 0.2 & na & na & na \\
\hline HR-SG-22 & 0.2 & 16.0 & 7.4 & 7.8 & 546 & 555 & 218 & 264 & 0.8 & na & na & na \\
\hline HR-SG-23 & 6.9 & 11.5 & 7.0 & 7.5 & 268 & 273 & 132 & 160 & 0.2 & na & na & na \\
\hline HR-SG-24 & 5.4 & 19.0 & 6.9 & 7.4 & 458 & 459 & 165 & 201 & 0.2 & na & na & na \\
\hline HR-SG-25 & 6.5 & 16.5 & $* 6.4$ & 7.3 & 248 & 249 & 110 & 134 & 0.1 & na & na & na \\
\hline HR-SG-26 & 3.5 & 15.5 & 7.2 & 7.7 & 378 & 374 & 178 & 216 & 0.5 & na & na & na \\
\hline HR-SG-27 & 5.9 & 15.0 & 7.0 & 7.3 & 232 & 245 & 124 & 151 & 0.1 & 118 & 143 & 0.2 \\
\hline HR-SG-28 & 7.7 & 15.0 & 6.6 & 7.1 & 300 & 305 & 141 & 172 & 0.1 & na & na & na \\
\hline HR-SG-29 & 0.8 & 11.5 & 7.1 & 7.5 & 444 & 455 & 213 & 259 & 0.4 & na & na & na \\
\hline HR-SG-30 & 9.4 & 12.0 & 7.2 & 7.6 & 372 & 388 & 199 & 242 & 0.4 & na & na & na \\
\hline HR-SG-31 & 0.6 & 15.5 & $* 6.3$ & 7.1 & 654 & 665 & 257 & 313 & 0.2 & 249 & 303 & 0.2 \\
\hline HR-SG-32 & - & 16.0 & $* 6.2$ & ${ }^{4} 8.4$ & 207 & 186 & 82.2 & 98 & 1.1 & na & na & na \\
\hline HR-SG-33 & 6.2 & 19.0 & 7.4 & 7.5 & 858 & 872 & 213 & 259 & 0.4 & na & na & na \\
\hline HR-SGU-01 & 1.6 & 22.0 & 7.4 & 7.7 & 844 & 866 & 361 & 438 & 1.0 & na & na & na \\
\hline HR-SGU-02 & ${ }^{5} 16.3$ & 15.5 & 7.6 & 7.8 & 192 & 198 & 96.9 & 117 & 0.3 & na & na & na \\
\hline HR-SGU-03 & 8.9 & 13.0 & 7.4 & 8.2 & 248 & 254 & 96.1 & 115 & 0.8 & na & na & na \\
\hline HR-SGU-04 & 6.3 & 14.5 & 6.6 & 7.4 & 394 & 401 & 168 & 204 & 0.2 & na & na & na \\
\hline
\end{tabular}


Table 4. Water-quality indicators in samples collected for the Santa Cruz, San Gabriel, and Peninsular Ranges Hard Rock Aquifers (Hard Rock) study unit, March 2011 through March 2012, California GAMA Priority Basin Project.-Continued

[The five-digit U.S. Geological Survey (USGS) parameter code below the constituent name is used to uniquely identify a specific constituent or property. Field alkalinities were measured at only 15 percent of the Hard Rock sites. GAMA well identification number: HR-SC, Santa Cruz study area grid well; HR-SG, San Gabriel study area grid well; HR-SGU, San Gabriel study area understanding well; HR-PR, Peninsular Ranges study area grid well; HR-PRFP, Peninsular Ranges study area flow-path well; HR-PRU, Peninsular Ranges study area understanding well. Reporting level, benchmark type, and benchmark level as of July 6, 2012. Benchmark type: Maximum contamination level benchmarks are listed as SMCL-CA when the SMCL-CA exists, and as the SMCL-US when no SMCL-CA exists. SMCL-US,

U.S. Environmental Protection Agency secondary maximum contaminant level; SMCL-CA, California Department of Public Health secondary maximum contaminant level. Other abbreviations: ${ }^{\circ} \mathrm{C}$, degrees Celsius; mg/L, milligrams per liter; na, not available; $\mu \mathrm{S} / \mathrm{cm}$, microsiemens per centimeter; <, less than; >, greater than; $\mathrm{CaCO}_{3}$, calcium carbonate; $\mathrm{HCO}_{3}^{-}$, bicarbonate; $\mathrm{CO}_{3}^{2-}$, carbonate; *, value greater than benchmark level or outside range; **, value greater than upper benchmark level; - , not detected]

\begin{tabular}{|c|c|c|c|c|c|c|c|c|c|c|c|c|}
\hline $\begin{array}{c}\text { GAMA well } \\
\text { identification } \\
\text { number }\end{array}$ & $\begin{array}{c}\text { Dissolved } \\
\text { oxygen, } \\
\text { field } \\
(\mathrm{mg} / \mathrm{L}) \\
(00300)\end{array}$ & $\begin{array}{c}\text { Water } \\
\text { temperature, } \\
\text { field } \\
\left({ }^{\circ} \mathrm{C}\right) \\
(00010)\end{array}$ & $\begin{array}{c}\text { pH, field } \\
\text { (standard } \\
\text { units) } \\
(00400)\end{array}$ & $\begin{array}{c}\text { pH, } \\
\text { laboratory } \\
\text { (standard } \\
\text { units) } \\
(00403)\end{array}$ & $\begin{array}{c}\text { Specific } \\
\text { conductance, } \\
\text { field } \\
(\mu \mathrm{S} / \mathrm{cm} \text { at } \\
\left.25^{\circ} \mathrm{C}\right) \\
(00095)\end{array}$ & $\begin{array}{c}\text { Specific } \\
\text { conductance, } \\
\text { laboratory } \\
(\mu \mathrm{S} / \mathrm{cm} \text { at } \\
\left.25^{\circ} \mathrm{C}\right) \\
(90095)\end{array}$ & $\begin{array}{c}\text { Alkalinity, } \\
\text { laboratory } \\
\text { (mg/L as } \\
\mathrm{CaCO}_{3} \text { ) } \\
(29801)\end{array}$ & $\begin{array}{c}\text { Bicarbonate, } \\
\text { laboratory } \\
(\mathrm{mg} / \mathrm{L} \text { as } \\
\left.\mathrm{HCO}_{3}^{-}\right)^{1}\end{array}$ & $\begin{array}{c}\text { Carbonate, } \\
\text { laboratory } \\
\text { (mg/L as } \\
\left.\mathrm{CO}_{3}^{2-}\right)^{1}\end{array}$ & $\begin{array}{l}\text { Alkalinity, } \\
\text { field } \\
\text { (mg/L as } \\
\left.\mathrm{CaCO}_{3}\right) \\
(29802)\end{array}$ & $\begin{array}{c}\text { Bicarbonate, } \\
\text { field } \\
\text { (mg/L as } \\
\mathrm{HCO}_{3}^{-} \text {) } \\
(63786)\end{array}$ & $\begin{array}{c}\text { Carbonate, } \\
\text { field } \\
\text { (mg/L as } \\
\left.\mathrm{CO}_{3}^{2-}\right) \\
(63788)\end{array}$ \\
\hline Benchmark type & na & na & SMCL-US & SMCL-US & SMCL-CA & SMCL-CA & na & na & na & na & na & na \\
\hline Benchmark level & na & na & $<6.5$ or $>8.5$ & $<6.5$ or $>8.5$ & ${ }^{2} 900(1,600)$ & ${ }^{2} 900(1,600)$ & na & na & na & na & na & na \\
\hline $\begin{array}{l}\text { [Reporting level } \\
\text { or range] }\end{array}$ & {$[0.2]$} & {$[0.0-38.5]$} & {$[0-14]$} & {$[0-14]$} & {$[5]$} & [5] & {$[4.6]$} & [1] & {$[0.1]$} & [1] & [1] & {$[0.1]$} \\
\hline \multicolumn{13}{|c|}{ Peninsular Ranges study area (50 wells sampled) } \\
\hline HR-PR-01 & 0.4 & 17.5 & 6.7 & 7.0 & 840 & 860 & 276 & 336 & 0.2 & na & na & na \\
\hline HR-PR-02 & 5.2 & 17.5 & 7.0 & 7.2 & 560 & 559 & 158 & 192 & 0.2 & na & na & na \\
\hline HR-PR-03 & - & 13.5 & 7.1 & 7.4 & 206 & 211 & 84.8 & 103 & 0.1 & na & na & na \\
\hline HR-PR-04 & - & 18.0 & 6.7 & 7.1 & 327 & 337 & 129 & 157 & - & na & na & na \\
\hline HR-PR-05 & 1.7 & 16.5 & 7.5 & 7.9 & 232 & 235 & 96.4 & 117 & 0.4 & 92.2 & 112 & 0.3 \\
\hline HR-PR-06 & - & 20.5 & 7.3 & 7.7 & 888 & 888 & 231 & 281 & 0.7 & na & na & na \\
\hline HR-PR-07 & 0.7 & 20.0 & 6.8 & 7.2 & $* * 2,360$ & $* * 2,310$ & 323 & 393 & 0.3 & na & na & na \\
\hline HR-PR-08 & 1.3 & 22.0 & 7.6 & 7.9 & 409 & 409 & 125 & 151 & 0.5 & na & na & na \\
\hline HR-PR-09 & 0.5 & 13.0 & 7.1 & 7.4 & 347 & 354 & 139 & 169 & 0.2 & 139 & 169 & 0.2 \\
\hline HR-PR-10 & 7.4 & 15.0 & * 5.8 & * 6.3 & 726 & 764 & 85.8 & 105 & - & na & na & na \\
\hline HR-PR-11 & 0.4 & 24.0 & 7.1 & 7.5 & 724 & 700 & 136 & 165 & 0.2 & na & na & na \\
\hline HR-PR-12 & 3.1 & 19.5 & 6.6 & 7.2 & * 901 & * 906 & 118 & 144 & - & na & na & na \\
\hline HR-PR-13 & 0.3 & 19.0 & 6.8 & 7.3 & $* 1,450$ & $* 1,450$ & 311 & 379 & 0.4 & na & na & na \\
\hline HR-PR-14 & 3.5 & 20.5 & 6.6 & 7.2 & 647 & 650 & 177 & 216 & 0.1 & 177 & 216 & 0.1 \\
\hline HR-PR-15 & 3.4 & 11.0 & * 6.2 & 6.7 & 201 & 206 & 78.5 & 95.7 & - & na & na & na \\
\hline
\end{tabular}


Table 4. Water-quality indicators in samples collected for the Santa Cruz, San Gabriel, and Peninsular Ranges Hard Rock Aquifers (Hard Rock) study unit, March 2011 through March 2012, California GAMA Priority Basin Project.-Continued

[The five-digit U.S. Geological Survey (USGS) parameter code below the constituent name is used to uniquely identify a specific constituent or property. Field alkalinities were measured at only 15 percent of the Hard Rock sites. GAMA well identification number: HR-SC, Santa Cruz study area grid well; HR-SG, San Gabriel study area grid well; HR-SGU, San Gabriel study area understanding well; HR-PR, Peninsular Ranges study area grid well; HR-PRFP, Peninsular Ranges study area flow-path well; HR-PRU, Peninsular Ranges study area understanding well. Reporting level, benchmark type, and benchmark level as of July 6, 2012. Benchmark type: Maximum contamination level benchmarks are listed as SMCL-CA when the SMCL-CA exists, and as the SMCL-US when no SMCL-CA exists. SMCL-US,

U.S. Environmental Protection Agency secondary maximum contaminant level; SMCL-CA, California Department of Public Health secondary maximum contaminant level. Other abbreviations: ${ }^{\circ} \mathrm{C}$, degrees Celsius; $\mathrm{mg} / \mathrm{L}$, milligrams per liter; na, not available; $\mu \mathrm{S} / \mathrm{cm}$, microsiemens per centimeter; $<$, less than; >, greater than; $\mathrm{CaCO}_{3}$, calcium carbonate; $\mathrm{HCO}_{3}^{-}$, bicarbonate; $\mathrm{CO}_{3}{ }^{2-}$, carbonate; *, value greater than benchmark level or outside range; ${ }^{* *}$, value greater than upper benchmark level; - , not detected]

\begin{tabular}{|c|c|c|c|c|c|c|c|c|c|c|c|c|}
\hline $\begin{array}{c}\text { GAMA well } \\
\text { identification } \\
\text { number }\end{array}$ & $\begin{array}{l}\text { Dissolved } \\
\text { oxygen, } \\
\text { field } \\
(\mathrm{mg} / \mathrm{L}) \\
(00300)\end{array}$ & $\begin{array}{c}\text { Water } \\
\text { temperature, } \\
\text { field } \\
\left({ }^{\circ} \mathrm{C}\right) \\
(00010)\end{array}$ & $\begin{array}{c}\text { pH, field } \\
\text { (standard } \\
\text { units) } \\
(00400)\end{array}$ & $\begin{array}{c}\text { pH, } \\
\text { laboratory } \\
\text { (standard } \\
\text { units) } \\
(00403)\end{array}$ & $\begin{array}{c}\text { Specific } \\
\text { conductance, } \\
\text { field } \\
(\mu \mathrm{S} / \mathrm{cm} \text { at } \\
\left.25^{\circ} \mathrm{C}\right) \\
(00095) \\
\end{array}$ & $\begin{array}{c}\text { Specific } \\
\text { conductance, } \\
\text { laboratory } \\
(\mu \mathrm{S} / \mathrm{cm} \text { at } \\
\left.25^{\circ} \mathrm{C}\right) \\
(90095)\end{array}$ & $\begin{array}{c}\text { Alkalinity, } \\
\text { laboratory } \\
\text { (mg/L as } \\
\left.\mathrm{CaCO}_{3}\right) \\
(29801)\end{array}$ & $\begin{array}{c}\text { Bicarbonate, } \\
\text { laboratory } \\
(\mathrm{mg} / \mathrm{L} \text { as } \\
\left.\mathrm{HCO}_{3}^{-}\right)^{1}\end{array}$ & $\begin{array}{c}\text { Carbonate, } \\
\text { laboratory } \\
\text { (mg/L as } \\
\left.\mathrm{CO}_{3}{ }^{2-}\right)^{1}\end{array}$ & $\begin{array}{l}\text { Alkalinity, } \\
\text { field } \\
\text { (mg/L as } \\
\left.\mathrm{CaCO}_{3}\right) \\
(29802)\end{array}$ & $\begin{array}{c}\text { Bicarbonate, } \\
\text { field } \\
\text { (mg/L as } \\
\left.\mathrm{HCO}_{3}^{-}\right) \\
(63786)\end{array}$ & $\begin{array}{c}\text { Carbonate, } \\
\text { field } \\
\text { (mg/L as } \\
\left.\mathrm{CO}_{3}^{2-}\right) \\
(63788)\end{array}$ \\
\hline Benchmark type & na & na & SMCL-US & SMCL-US & SMCL-CA & SMCL-CA & na & na & na & na & na & na \\
\hline Benchmark level & na & na & $<6.5$ or $>8.5$ & $<6.5$ or $>8.5$ & ${ }^{2} 900(1,600)$ & ${ }^{2} 900(1,600)$ & na & na & na & na & na & na \\
\hline $\begin{array}{l}\text { [Reporting level } \\
\text { or range] }\end{array}$ & {$[0.2]$} & {$[0.0-38.5]$} & [0-14] & {$[0-14]$} & [5] & [5] & [4.6] & [1] & {$[0.1]$} & [1] & [1] & {$[0.1]$} \\
\hline \multicolumn{13}{|c|}{ Peninsular Ranges study area (50 wells sampled)—Continued } \\
\hline HR-PR-16 & 0.4 & 22.5 & 6.9 & 7.2 & $* 1,260$ & $* 1,240$ & 186 & 226 & 0.2 & 185 & 225 & 0.2 \\
\hline HR-PR-17 & 3.5 & 18.5 & 7.0 & 7.2 & 597 & 595 & 193 & 235 & 0.2 & na & na & na \\
\hline HR-PR-18 & 0.3 & 22.0 & 6.8 & 7.1 & * 918 & * 926 & 346 & 421 & 0.2 & na & na & na \\
\hline HR-PR-19 & 0.8 & 16.5 & $* 6.0$ & 6.8 & 422 & 458 & 164 & 199 & - & na & na & na \\
\hline HR-PR-20 & 5.1 & 19.0 & 6.8 & 7.0 & 526 & 539 & 150 & 182 & - & na & na & na \\
\hline HR-PR-21 & 0.6 & 22.0 & 6.9 & 7.2 & $* 1,090$ & $* 1,160$ & 215 & 261 & 0.2 & na & na & na \\
\hline HR-PR-22 & 0.5 & 18.0 & 7.1 & 7.3 & 832 & 882 & 267 & 325 & 0.3 & na & na & na \\
\hline HR-PR-23 & 0.4 & 20.0 & 7.0 & 7.2 & 525 & 533 & 177 & 215 & 0.2 & na & na & na \\
\hline HR-PR-24 & 3.4 & 21.5 & $* 6.4$ & 6.6 & $* 1,190$ & $* 1,210$ & 132 & 161 & - & na & na & na \\
\hline HR-PR-25 & 6.5 & 21.0 & 7.3 & 7.5 & * 967 & * 988 & 289 & 351 & 0.6 & 278 & 338 & 0.4 \\
\hline HR-PR-26 & 0.4 & 18.0 & 6.7 & 7.0 & $* * 2,760$ & $* * 2,800$ & 320 & 390 & 0.2 & na & na & na \\
\hline HR-PR-27 & 5.6 & 15.0 & 6.9 & 7.3 & 211 & 215 & 81.6 & 99.3 & - & na & na & na \\
\hline HR-PR-28 & 0.7 & 21.0 & 7.5 & 7.3 & $* 1,220$ & $* 1,220$ & 192 & 233 & 0.2 & na & na & na \\
\hline HR-PR-29 & - & 25.5 & $* 9.5$ & * 9.2 & 303 & 311 & 58.3 & 61.8 & 4.1 & 56.2 & 55.5 & 5.9 \\
\hline HR-PR-30 & 3.1 & 15.0 & 6.5 & 6.9 & 299 & 304 & 123 & 150 & - & na & na & na \\
\hline
\end{tabular}


Table 4. Water-quality indicators in samples collected for the Santa Cruz, San Gabriel, and Peninsular Ranges Hard Rock Aquifers (Hard Rock) study unit, March 2011 through March 2012, California GAMA Priority Basin Project.—Continued

[The five-digit U.S. Geological Survey (USGS) parameter code below the constituent name is used to uniquely identify a specific constituent or property. Field alkalinities were measured at only 15 percent of the Hard Rock sites. GAMA well identification number: HR-SC, Santa Cruz study area grid well; HR-SG, San Gabriel study area grid well; HR-SGU, San Gabriel study area understanding well; HR-PR, Peninsular Ranges study area grid well; HR-PRFP, Peninsular Ranges study area flow-path well; HR-PRU, Peninsular Ranges study area understanding well. Reporting level, benchmark type, and benchmark level as of July 6, 2012. Benchmark type: Maximum contamination level benchmarks are listed as SMCL-CA when the SMCL-CA exists, and as the SMCL-US when no SMCL-CA exists. SMCL-US,

U.S. Environmental Protection Agency secondary maximum contaminant level; SMCL-CA, California Department of Public Health secondary maximum contaminant level. Other abbreviations: ${ }^{\circ} \mathrm{C}$, degrees Celsius; mg/L, milligrams per liter; na, not available; $\mu \mathrm{S} / \mathrm{cm}$, microsiemens per centimeter; $<$, less than; >, greater than; $\mathrm{CaCO}_{3}$, calcium carbonate; $\mathrm{HCO}_{3}^{-}$, bicarbonate; $\mathrm{CO}_{3}{ }^{2-}$, carbonate; *, value greater than benchmark level or outside range; ${ }^{* *}$, value greater than upper benchmark level; —, not detected]

\begin{tabular}{|c|c|c|c|c|c|c|c|c|c|c|c|c|}
\hline $\begin{array}{c}\text { GAMA well } \\
\text { identification } \\
\text { number }\end{array}$ & $\begin{array}{l}\text { Dissolved } \\
\text { oxygen, } \\
\text { field } \\
(\mathrm{mg} / \mathrm{L}) \\
(00300)\end{array}$ & $\begin{array}{c}\text { Water } \\
\text { temperature, } \\
\text { field } \\
\left({ }^{\circ} \mathrm{C}\right) \\
(00010)\end{array}$ & $\begin{array}{c}\text { pH, field } \\
\text { (standard } \\
\text { units) } \\
(00400)\end{array}$ & $\begin{array}{c}\text { pH, } \\
\text { laboratory } \\
\text { (standard } \\
\text { units) } \\
(00403)\end{array}$ & $\begin{array}{c}\text { Specific } \\
\text { conductance, } \\
\text { field } \\
(\mu \mathrm{S} / \mathrm{cm} \text { at } \\
\left.25^{\circ} \mathrm{C}\right) \\
(00095)\end{array}$ & $\begin{array}{c}\text { Specific } \\
\text { conductance, } \\
\text { laboratory } \\
(\mu \mathrm{S} / \mathrm{cm} \text { at } \\
\left.25^{\circ} \mathrm{C}\right) \\
(90095)\end{array}$ & $\begin{array}{c}\text { Alkalinity, } \\
\text { laboratory } \\
\text { (mg/L as } \\
\left.\mathrm{CaCO}_{3}\right) \\
(29801)\end{array}$ & $\begin{array}{c}\text { Bicarbonate, } \\
\text { laboratory } \\
\text { (mg/L as } \\
\left.\mathrm{HCO}_{3}^{-}\right)^{1}\end{array}$ & $\begin{array}{c}\text { Carbonate, } \\
\text { laboratory } \\
\text { (mg/L as } \\
\left.\mathrm{CO}_{3}{ }^{2-}\right)^{1}\end{array}$ & $\begin{array}{c}\text { Alkalinity, } \\
\text { field } \\
\text { (mg/L as } \\
\mathrm{CaCO}_{3} \text { ) } \\
(29802)\end{array}$ & $\begin{array}{c}\text { Bicarbonate, } \\
\text { field } \\
\text { (mg/L as } \\
\left.\mathrm{HCO}_{3}^{-}\right) \\
(63786)\end{array}$ & $\begin{array}{c}\text { Carbonate, } \\
\text { field } \\
\text { (mg/L as } \\
\left.\mathrm{CO}_{3}^{2-}\right) \\
(63788)\end{array}$ \\
\hline Benchmark type & na & na & SMCL-US & SMCL-US & SMCL-CA & SMCL-CA & na & na & na & na & na & na \\
\hline Benchmark level & na & na & $<6.5$ or $>8.5$ & $<6.5$ or $>8.5$ & ${ }^{2} 900(1,600)$ & ${ }^{2} 900(1,600)$ & na & na & na & na & na & na \\
\hline $\begin{array}{l}\text { [Reporting level } \\
\text { or range] }\end{array}$ & {$[0.2]$} & {$[0.0-38.5]$} & [0-14] & [0-14] & [5] & [5] & {$[4.6]$} & [1] & {$[0.1]$} & [1] & [1] & {$[0.1]$} \\
\hline \multicolumn{13}{|c|}{ Peninsular Ranges study area (50 wells sampled) - Continued } \\
\hline HR-PR-31 & 3.6 & 13.0 & 6.6 & 7.0 & 167 & 170 & 68 & 82.8 & - & na & na & na \\
\hline HR-PR-32 & 0.7 & 19.0 & 7.3 & 7.5 & $* 1,120$ & $* 1,160$ & 256 & 312 & 0.5 & na & na & na \\
\hline HR-PR-33 & 3.5 & 17.5 & 6.7 & 6.9 & 645 & 682 & 179 & 218 & - & na & na & na \\
\hline HR-PR-34 & 4.6 & 17.0 & 7.4 & 7.6 & 762 & 775 & 275 & 334 & 0.6 & na & na & na \\
\hline HR-PR-35 & 2.8 & 17.0 & 7.4 & 7.7 & 297 & 308 & 130 & 158 & 0.3 & na & na & na \\
\hline HR-PR-36 & 1.3 & 11.0 & 6.9 & 7.2 & 218 & 224 & 110 & 134 & - & na & na & na \\
\hline HR-PRFP-01 & 0.6 & 17.5 & 7.3 & 7.4 & 539 & 552 & 140 & 170 & 0.2 & na & na & na \\
\hline HR-PRFP-02 & 4.2 & 24.0 & 7.1 & 7.2 & $* 1,370$ & $* 1,390$ & 193 & 235 & 0.2 & na & na & na \\
\hline HR-PRFP-03 & 0.3 & 19.0 & 7.1 & 7.3 & 794 & 802 & 164 & 200 & 0.2 & 160 & 195 & 0.1 \\
\hline HR-PRFP-04 & 3.2 & 23.0 & 6.9 & 7.1 & 835 & 836 & 137 & 167 & - & na & na & na \\
\hline HR-PRFP-05 & 0.8 & 22.5 & 7.1 & 7.2 & $* 1,310$ & $* 1,350$ & 296 & 361 & 0.3 & na & na & na \\
\hline HR-PRFP-06 & 1.0 & 22.5 & 7.3 & 7.4 & 864 & 888 & 216 & 263 & 0.3 & na & na & na \\
\hline HR-PRFP-07 & 0.3 & 17.0 & 7.5 & 7.7 & 441 & 445 & 108 & 131 & 0.3 & na & na & na \\
\hline HR-PRFP-08 & 0.2 & 18.0 & $* 9.1$ & $* 8.9$ & 211 & 206 & 55.5 & 62.0 & 2.5 & na & na & na \\
\hline HR-PRFP-09 & 2.1 & 23.0 & 7.0 & 7.3 & $* * 2,020$ & $* * 1,940$ & 322 & 393 & 0.3 & na & na & na \\
\hline
\end{tabular}


Table 4. Water-quality indicators in samples collected for the Santa Cruz, San Gabriel, and Peninsular Ranges Hard Rock Aquifers (Hard Rock) study unit, March 2011 through March 2012, California GAMA Priority Basin Project.-Continued

The five-digit U.S. Geological Survey (USGS) parameter code below the constituent name is used to uniquely identify a specific constituent or property. Field alkalinities were measured at only 15 percent of the Hard Rock sites. GAMA well identification number: HR-SC, Santa Cruz study area grid well; HR-SG, San Gabriel study area grid well; HR-SGU, San Gabriel study area understanding well; HR-PR, Peninsular Ranges study area grid well; HR-PRFP, Peninsular Ranges study area flow-path well; HR-PRU, Peninsular Ranges study area understanding well. Reporting level, benchmark type, and benchmark level as of July 6, 2012. Benchmark type: Maximum contamination level benchmarks are listed as SMCL-CA when the SMCL-CA exists, and as the SMCL-US when no SMCL-CA exists. SMCL-US,

U.S. Environmental Protection Agency secondary maximum contaminant level; SMCL-CA, California Department of Public Health secondary maximum contaminant level. Other abbreviations: ${ }^{\circ} \mathrm{C}$, degrees Celsius; $\mathrm{mg} / \mathrm{L}$, milligrams per liter; na, not available; $\mu \mathrm{S} / \mathrm{cm}$, microsiemens per centimeter; <, less than; >, greater than; $\mathrm{CaCO}_{3}$, calcium carbonate; $\mathrm{HCO}_{3}{ }^{-}$, bicarbonate; $\mathrm{CO}_{3}{ }^{2-}$, carbonate; *, value greater than benchmark level or outside range; $* *$, value greater than upper benchmark level; - , not detected]

\begin{tabular}{|c|c|c|c|c|c|c|c|c|c|c|c|c|}
\hline $\begin{array}{c}\text { GAMA well } \\
\text { identification } \\
\text { number }\end{array}$ & $\begin{array}{c}\text { Dissolved } \\
\text { oxygen, } \\
\text { field } \\
\text { (mg/L) } \\
(00300)\end{array}$ & $\begin{array}{c}\text { Water } \\
\text { temperature, } \\
\text { field } \\
\left({ }^{\circ} \mathrm{C}\right) \\
(00010)\end{array}$ & $\begin{array}{c}\text { pH, field } \\
\text { (standard } \\
\text { units) } \\
(00400)\end{array}$ & $\begin{array}{c}\text { pH, } \\
\text { laboratory } \\
\text { (standard } \\
\text { units) } \\
\text { (00403) }\end{array}$ & $\begin{array}{c}\text { Specific } \\
\text { conductance, } \\
\text { field } \\
(\mu \mathrm{S} / \mathrm{cm} \text { at } \\
\left.25^{\circ} \mathrm{C}\right) \\
(00095)\end{array}$ & $\begin{array}{l}\text { Specific } \\
\text { conductance, } \\
\text { laboratory } \\
(\mu \mathrm{S} / \mathrm{cm} \text { at } \\
\left.25^{\circ} \mathrm{C}\right) \\
(90095)\end{array}$ & $\begin{array}{c}\text { Alkalinity, } \\
\text { laboratory } \\
\text { (mg/L as } \\
\left.\mathrm{CaCO}_{3}\right) \\
(29801)\end{array}$ & $\begin{array}{c}\text { Bicarbonate, } \\
\text { laboratory } \\
(\mathrm{mg} / \mathrm{L} \text { as } \\
\left.\mathrm{HCO}_{3}^{-}\right)^{1}\end{array}$ & $\begin{array}{c}\text { Carbonate, } \\
\text { laboratory } \\
\text { (mg/L as } \\
\left.\mathrm{CO}_{3}{ }^{2-}\right)^{1}\end{array}$ & $\begin{array}{c}\text { Alkalinity, } \\
\text { field } \\
\text { (mg/L as } \\
\left.\mathrm{CaCO}_{3}\right) \\
(29802)\end{array}$ & $\begin{array}{c}\text { Bicarbonate, } \\
\text { field } \\
\text { (mg/L as } \\
\left.\mathrm{HCO}_{3}^{-}\right) \\
(63786)\end{array}$ & $\begin{array}{c}\text { Carbonate, } \\
\text { field } \\
(\mathrm{mg} / \mathrm{L} \text { as } \\
\left.\mathrm{CO}_{3}^{2-}\right) \\
(63788)\end{array}$ \\
\hline Benchmark type & na & na & SMCL-US & SMCL-US & SMCL-CA & SMCL-CA & na & na & na & na & na & na \\
\hline Benchmark level & na & na & $<6.5$ or $>8.5$ & $<6.5$ or $>8.5$ & $2900(1,600)$ & $2900(1,600)$ & na & na & na & na & na & na \\
\hline $\begin{array}{l}\text { [Reporting level } \\
\text { or range] }\end{array}$ & {$[0.2]$} & {$[0.0-38.5]$} & {$[0-14]$} & {$[0-14]$} & [5] & [5] & [4.6] & [1] & [0.1] & [1] & [1] & {$[0.1]$} \\
\hline \multicolumn{13}{|c|}{ Peninsular Ranges study area (50 wells sampled)_-Continued } \\
\hline HR-PRFP-10 & 0.5 & 17.5 & 6.7 & 7.1 & 484 & 488 & 138 & 168 & 0.1 & 136 & 166 & 0.1 \\
\hline HR-PRFP-11 & 4.4 & 22.0 & 6.9 & 7.0 & $* * 4,400$ & $* * 4,750$ & 281 & 342 & 0.2 & na & na & na \\
\hline HR-PRFP-12 & 6.6 & 22.0 & 7.1 & 7.3 & $* 1,570$ & $* 1,580$ & 250 & 304 & 0.3 & na & na & na \\
\hline HR-PRFP-13 & 0.7 & 16.5 & 6.5 & 6.8 & $* * 3,480$ & $* * 3,530$ & 240 & 292 & - & na & na & na \\
\hline HR-PRU-01 & 1.2 & 12.0 & 6.9 & 7.3 & 232 & 238 & 102 & 124 & 0.1 & na & na & na \\
\hline
\end{tabular}

${ }^{1}$ Laboratory carbonate and bicarbonate results were calculated from laboratory alkalinity and $\mathrm{pH}$, according to the advanced speciation method. Results are stored in a separate database maintained by the GAMA Program.

${ }^{2}$ The SMCL-CA for specific conductance has recommended and upper benchmark values. The upper value is shown in parentheses.

${ }^{3}$ Values estimated by laboratory: high variability exists for $\mathrm{pH}$ when specific conductance is $<100 \mu \mathrm{S} / \mathrm{cm}$ at $25{ }^{\circ} \mathrm{C}$.

${ }^{4}$ High laboratory $\mathrm{pH}$ compared to field value. Field $\mathrm{pH}$ is considered more representative of aquifer conditions; laboratory $\mathrm{pH}$ can be affected by reactions that occur in the sample between the time of collection and analysis, which was 60 days for this sample.

${ }^{5}$ Sample was collected from a spring and was highly oxic, likely indicating exposure to the atmosphere prior to sampling. 
Table 5. Volatile organic compounds (VOCs) detected in samples collected for the Santa Cruz, San Gabriel, and Peninsular Ranges Hard Rock Aquifers (Hard Rock) study unit, March 2011 through March 2012, California GAMA Priority Basin Project.

[The five-digit U.S. Geological Survey (USGS) parameter code below the constituent name is used to uniquely identify a specific constituent or property. Samples from 110 wells were analyzed, but only samples with detections of at least one constituent are listed. Constituents are grouped by primary use or source and listed in order of decreasing detection frequency for each group in the 94 grid wells sampled. All analytes are listed in table 3A. GAMA well identification number: HR-SC, Santa Cruz study area grid well; HR-SG, San Gabriel study area grid well; HR-SGU, San Gabriel study area understanding well; HR-PR, Peninsular Ranges study area grid well; HR-PRFP, Peninsular Ranges study area flow-path well; HR-PRU, Peninsular Ranges study area understanding well. Reporting levels, benchmark types, and benchmark levels as of July 6, 2012. Benchmark type: Maximum contaminant level benchmarks are listed as MCL-US when the MCL-US and MCL-CA are identical, and as MCL-CA when the MCL-CA is lower than the MCL-US or no MCL-US exists. MCL-US, U.S. Environmental Protection Agency maximum contaminant level; MCL-CA, California Department of Public Health maximum contaminant level; NL-CA, CDPH notification level. Other abbreviations: E, estimated or having a higher degree of uncertainty; LRL, laboratory reporting level; $\mu \mathrm{g} / \mathrm{L}$, micrograms per liter; na, not available; - , not detected]

\begin{tabular}{|c|c|c|c|c|c|c|c|c|c|c|c|c|}
\hline \multirow[b]{2}{*}{$\begin{array}{c}\text { GAMA } \\
\text { well } \\
\text { identification } \\
\text { number }\end{array}$} & \multicolumn{4}{|c|}{ Disinfection byproducts } & \multicolumn{8}{|c|}{ Solvents } \\
\hline & 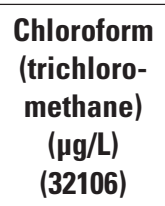 & $\begin{array}{c}\text { Bromo- } \\
\text { dichloro- } \\
\text { methane } \\
(\mu \mathrm{g} / \mathrm{L}) \\
(32101)\end{array}$ & $\begin{array}{c}\text { Dibromo- } \\
\text { chloro- } \\
\text { methane } \\
(\mu \mathrm{g} / \mathrm{L}) \\
(32105)\end{array}$ & $\begin{array}{c}\text { Bromoform } \\
\text { (tribromo- } \\
\text { methane) } \\
(\mu \mathrm{g} / \mathrm{L}) \\
(32104)\end{array}$ & $\begin{array}{c}\text { Tetra- } \\
\text { chloro- } \\
\text { ethene } \\
(\mu \mathrm{g} / \mathrm{L}) \\
(34475)\end{array}$ & $\begin{array}{c}\text { Trichloro- } \\
\text { ethene } \\
(\mu \mathrm{g} / \mathrm{L}) \\
(39180)\end{array}$ & $\begin{array}{c}\text { 1,1,1-Tri- } \\
\text { chloro- } \\
\text { ethane } \\
(\mu \mathrm{g} / \mathrm{L}) \\
(34506)\end{array}$ & $\begin{array}{c}\text { 1,2,4-Tri- } \\
\text { chloro- } \\
\text { benzene } \\
(\mu \mathrm{g} / \mathrm{L}) \\
(34551)\end{array}$ & $\begin{array}{c}\text { 1,3-Di- } \\
\text { chloro- } \\
\text { benzene } \\
(\mu \mathrm{g} / \mathrm{L}) \\
(34566)\end{array}$ & $\begin{array}{c}\text { 1,4-Di- } \\
\text { chloro- } \\
\text { benzene } \\
(\mu \mathrm{g} / \mathrm{L}) \\
(34571)\end{array}$ & $\begin{array}{c}\text { Carbon tetrachloride } \\
\text { (tetrachloro- } \\
\text { methane) } \\
(\mu \mathrm{g} / \mathrm{L}) \\
(32102)\end{array}$ & $\begin{array}{c}\text { Chloro- } \\
\text { benzene } \\
(\mu \mathrm{g} / \mathrm{L}) \\
(34301)\end{array}$ \\
\hline Benchmark type & MCL-US & MCL-US & MCL-US & MCL-US & MCL-US & MCL-US & MCL-US & MCL-CA & HAL-US & MCL-CA & MCL-CA & MCL-CA \\
\hline Benchmark level & ${ }^{2} 80$ & ${ }^{2} 80$ & ${ }^{2} 80$ & ${ }^{2} 80$ & 5 & 5 & 200 & 5 & 600 & 5 & 0.5 & 70 \\
\hline [LRL] & {$[0.03]$} & {$[0.034]$} & {$[0.12]$} & {$[0.1]$} & {$[0.026]$} & {$[0.022]$} & {$[0.030]$} & {$[0.08]$} & {$[0.024]$} & {$[0.026]$} & {$[0.06]$} & {$[0.026]$} \\
\hline \multicolumn{13}{|c|}{ Hard Rock study unit (94 grid wells sampled) } \\
\hline $\begin{array}{l}\text { Number of wells with } \\
\text { detections }\end{array}$ & 28 & 4 & 3 & 2 & 7 & 3 & 2 & 1 & 1 & 1 & 1 & 1 \\
\hline Detection frequency (percent) & 30 & 4.3 & 3.2 & 2.1 & 7.4 & 3.2 & 2.1 & 1.1 & 1.1 & 1.1 & 1.1 & 1.1 \\
\hline \multicolumn{13}{|c|}{ Santa Cruz study area ( 25 grid wells sampled) } \\
\hline HR-SC-02 & 0.02 & - & - & - & - & - & - & - & - & - & - & - \\
\hline HR-SC-04 & 0.02 & - & - & - & - & - & - & - & - & - & - & - \\
\hline HR-SC-08 & 0.72 & - & - & - & - & - & - & - & - & - & - & - \\
\hline HR-SC-11 & - & - & - & - & - & - & 0.018 & - & - & - & - & - \\
\hline HR-SC-13 & - & - & - & - & - & - & - & - & - & - & - & - \\
\hline HR-SC-14 & 0.02 & - & - & - & - & - & - & - & - & - & - & - \\
\hline HR-SC-16 ${ }^{4}$ & 20.4 & 6.59 & 2.00 & 0.22 & - & - & - & - & - & - & - & - \\
\hline HR-SC-17 & 0.12 & - & - & - & - & - & - & - & - & - & - & 0.369 \\
\hline HR-SC-20 & 0.43 & 0.043 & - & - & - & - & - & - & - & - & - & - \\
\hline HR-SC-21 & - & - & - & - & - & - & - & - & - & - & - & - \\
\hline
\end{tabular}


Table 5. Volatile organic compounds (VOCs) detected in samples collected for the Santa Cruz, San Gabriel, and Peninsular Ranges Hard Rock Aquifers (Hard Rock) study unit, March 2011 through March 2012, California GAMA Priority Basin Project.-Continued

[The five-digit U.S. Geological Survey (USGS) parameter code below the constituent name is used to uniquely identify a specific constituent or property. Samples from 110 wells were analyzed, but only samples with detections of at least one constituent are listed. Constituents are grouped by primary use or source and listed in order of decreasing detection frequency for each group in the 94 grid wells sampled. All analytes are listed in table 3A. GAMA well identification number: HR-SC, Santa Cruz study area grid well; HR-SG, San Gabriel study area grid well; HR-SGU, San Gabriel study area understanding well; HR-PR, Peninsular Ranges study area grid well; HR-PRFP, Peninsular Ranges study area flow-path well; HR-PRU, Peninsular Ranges study area understanding well. Reporting levels, benchmark types, and benchmark levels as of July 6, 2012. Benchmark type: Maximum contaminant level benchmarks are listed as MCL-US when the MCL-US and MCL-CA are identical, and as MCL-CA when the MCL-CA is lower than the MCL-US or no MCL-US exists. MCL-US, U.S. Environmental Protection Agency maximum contaminant level; MCL-CA, California Department of Public Health maximum contaminant level; NL-CA, CDPH notification level. Other abbreviations: E, estimated or having a higher degree of uncertainty; LRL, laboratory reporting level; $\mu \mathrm{g} / \mathrm{L}$, micrograms per liter; na, not available; - , not detected]

\begin{tabular}{|c|c|c|c|c|c|c|c|c|c|c|c|c|}
\hline \multirow[b]{2}{*}{$\begin{array}{c}\text { GAMA } \\
\text { well } \\
\text { identification } \\
\text { number }\end{array}$} & \multicolumn{4}{|c|}{ Disinfection byproducts } & \multicolumn{8}{|c|}{ Solvents } \\
\hline & $\begin{array}{l}\text { Chloroform } \\
\text { (trichloro- } \\
\text { methane) } \\
(\mu \mathrm{g} / \mathrm{L}) \\
(32106)\end{array}$ & $\begin{array}{l}\text { Bromo- } \\
\text { dichloro- } \\
\text { methane } \\
(\mu \mathrm{g} / \mathrm{L}) \\
(32101)\end{array}$ & $\begin{array}{c}\text { Dibromo- } \\
\text { chloro- } \\
\text { methane } \\
(\mu \mathrm{g} / \mathrm{L}) \\
(32105)\end{array}$ & $\begin{array}{c}\text { Bromoform } \\
\text { (tribromo- } \\
\text { methane) } \\
(\mu \mathrm{g} / \mathrm{L}) \\
(32104)\end{array}$ & $\begin{array}{c}\text { Tetra- } \\
\text { chloro- } \\
\text { ethene } \\
(\mu \mathrm{g} / \mathrm{L}) \\
(34475)\end{array}$ & $\begin{array}{l}\text { Trichloro- } \\
\text { ethene } \\
(\mu \mathrm{g} / \mathrm{L}) \\
(\mathbf{3 9 1 8 0})\end{array}$ & $\begin{array}{c}\text { 1,1,1-Tri- } \\
\text { chloro- } \\
\text { ethane } \\
(\mu \mathrm{g} / \mathrm{L}) \\
(34506)\end{array}$ & $\begin{array}{c}\text { 1,2,4-Tri- } \\
\text { chloro- } \\
\text { benzene } \\
(\mu \mathrm{g} / \mathrm{L}) \\
(34551)\end{array}$ & $\begin{array}{c}\text { 1,3-Di- } \\
\text { chloro- } \\
\text { benzene } \\
(\mu \mathrm{g} / \mathrm{L}) \\
(34566)\end{array}$ & $\begin{array}{c}\text { 1,4-Di- } \\
\text { chloro- } \\
\text { benzene } \\
(\mu \mathrm{g} / \mathrm{L}) \\
(34571)\end{array}$ & $\begin{array}{c}\text { Carbon tetrachloride } \\
\text { (tetrachloro- } \\
\text { methane) } \\
(\mu \mathrm{g} / \mathrm{L}) \\
(32102)\end{array}$ & $\begin{array}{c}\text { Chloro- } \\
\text { benzene } \\
(\mu \mathrm{g} / \mathrm{L}) \\
(34301)\end{array}$ \\
\hline Benchmark type & MCL-US & MCL-US & MCL-US & MCL-US & MCL-US & MCL-US & MCL-US & MCL-CA & HAL-US & MCL-CA & MCL-CA & MCL-CA \\
\hline Benchmark level & 280 & 280 & 280 & 280 & 5 & 5 & 200 & 5 & 600 & 5 & 0.5 & 70 \\
\hline [LRL] & {$[0.03]$} & {$[0.034]$} & {$[0.12]$} & {$[0.1]$} & {$[0.026]$} & {$[0.022]$} & {$[0.030]$} & {$[0.08]$} & {$[0.024]$} & {$[0.026]$} & {$[0.06]$} & {$[0.026]$} \\
\hline \multicolumn{13}{|c|}{ Santa Cruz study area (25 grid wells sampled)—Continued } \\
\hline HR-SC-22 & - & - & - & - & - & - & - & - & - & - & - & - \\
\hline HR-SC-23 & - & - & - & - & - & - & - & - & - & - & - & - \\
\hline HR-SC-24 & 0.02 & - & - & - & - & - & - & - & - & - & - & - \\
\hline HR-SC-25 & 0.10 & - & - & - & 0.014 & 0.084 & 0.043 & - & - & - & - & - \\
\hline $\begin{array}{l}\text { Number of wells with } \\
\text { detections }\end{array}$ & 9 & 2 & 1 & 1 & 1 & 1 & 2 & 0 & 0 & 0 & 0 & 1 \\
\hline Detection frequency (percent) & 36 & 8.0 & 4.0 & 4.0 & 4.0 & 4.0 & 8.0 & 0 & 0 & 0 & 0 & 4.0 \\
\hline \multicolumn{13}{|c|}{ San Gabriel study area (33 grid wells sampled) } \\
\hline HR-SG-02 & 0.08 & - & - & - & - & - & - & - & - & - & - & - \\
\hline HR-SG-04 & - & - & - & - & 0.019 & 0.040 & - & - & - & - & - & - \\
\hline HR-SG-05 & - & - & - & - & 0.027 & - & - & - & - & - & - & - \\
\hline HR-SG-08 & 0.04 & 0.035 & 0.06 & - & - & - & - & - & - & - & - & - \\
\hline HR-SG-15 & 0.03 & 0.039 & 0.08 & 0.06 & - & - & - & - & - & - & - & - \\
\hline HR-SG-27 & 0.02 & - & - & - & 0.024 & 0.428 & - & 0.17 & 0.078 & 0.016 & - & - \\
\hline HR-SG-28 & 0.20 & - & - & - & - & - & - & - & - & - & - & - \\
\hline HR-SG-31 & 0.08 & - & - & - & - & - & - & - & - & - & - & - \\
\hline HR-SG-33 & 0.32 & - & - & - & - & - & - & - & - & - & - & - \\
\hline $\begin{array}{l}\text { Number of wells with } \\
\text { detections }\end{array}$ & 7 & 2 & 2 & 1 & 3 & 2 & 0 & 1 & 1 & 1 & 0 & 0 \\
\hline Detection frequency (percent) & 21 & 6.1 & 6.1 & 3.0 & 9.1 & 6.1 & 0 & 3.0 & 3.0 & 3.0 & 0 & 0 \\
\hline
\end{tabular}


Table 5. Volatile organic compounds (VOCs) detected in samples collected for the Santa Cruz, San Gabriel, and Peninsular Ranges Hard Rock Aquifers (Hard Rock) study unit, March 2011 through March 2012, California GAMA Priority Basin Project.-Continued

[The five-digit U.S. Geological Survey (USGS) parameter code below the constituent name is used to uniquely identify a specific constituent or property. Samples from 110 wells were analyzed, but only samples with detections of at least one constituent are listed. Constituents are grouped by primary use or source and listed in order of decreasing detection frequency for each group in the 94 grid wells sampled. All analytes are listed in table 3A. GAMA well identification number: HR-SC, Santa Cruz study area grid well; HR-SG, San Gabriel study area grid well; HR-SGU, San Gabriel study area understanding well; HR-PR, Peninsular Ranges study area grid well; HR-PRFP, Peninsular Ranges study area flow-path well; HR-PRU, Peninsular Ranges study area understanding well. Reporting levels, benchmark types, and benchmark levels as of July 6, 2012. Benchmark type: Maximum contaminant level benchmarks are listed as MCL-US when the MCL-US and MCL-CA are identical, and as MCL-CA when the MCL-CA is lower than the MCL-US or no MCL-US exists. MCL-US, U.S. Environmental Protection Agency maximum contaminant level; MCL-CA, California Department of Public Health maximum contaminant level; NL-CA, CDPH notification level. Other abbreviations: E, estimated or having a higher degree of uncertainty; LRL, laboratory reporting level; $\mu \mathrm{g} / \mathrm{L}$, micrograms per liter; na, not available; - , not detected]

\begin{tabular}{|c|c|c|c|c|c|c|c|c|c|c|c|c|}
\hline \multirow[b]{2}{*}{$\begin{array}{c}\text { GAMA } \\
\text { well } \\
\text { identification } \\
\text { number }\end{array}$} & \multicolumn{4}{|c|}{ Disinfection byproducts } & \multicolumn{8}{|c|}{ Solvents } \\
\hline & $\begin{array}{c}\text { Chloroform } \\
\text { (trichloro- } \\
\text { methane) } \\
(\mu \mathrm{g} / \mathrm{L}) \\
(32106) \\
\end{array}$ & $\begin{array}{c}\text { Bromo- } \\
\text { dichloro- } \\
\text { methane } \\
(\mu \mathrm{g} / \mathrm{L}) \\
(32101) \\
\end{array}$ & $\begin{array}{c}\text { Dibromo- } \\
\text { chloro- } \\
\text { methane } \\
(\mu \mathrm{g} / \mathrm{L}) \\
(32105)\end{array}$ & $\begin{array}{c}\text { Bromoform } \\
\text { (tribromo- } \\
\text { methane) } \\
(\mu \mathrm{g} / \mathrm{L}) \\
(32104)\end{array}$ & $\begin{array}{l}\text { Tetra- } \\
\text { chloro- } \\
\text { ethene } \\
(\mu \mathrm{g} / \mathrm{L}) \\
(34475)\end{array}$ & $\begin{array}{c}\text { Trichloro- } \\
\text { ethene } \\
(\mu \mathrm{g} / \mathrm{L}) \\
(39180)\end{array}$ & $\begin{array}{c}\text { 1,1,1-Tri- } \\
\text { chloro- } \\
\text { ethane } \\
(\mu \mathrm{g} / \mathrm{L}) \\
(34506)\end{array}$ & $\begin{array}{c}1,2,4-\text { Tri- } \\
\text { chloro- } \\
\text { benzene } \\
(\mu \mathrm{g} / \mathrm{L}) \\
(34551)\end{array}$ & $\begin{array}{c}\text { 1,3-Di- } \\
\text { chloro- } \\
\text { benzene } \\
(\mu \mathrm{g} / \mathrm{L}) \\
(34566)\end{array}$ & $\begin{array}{c}\text { 1,4-Di- } \\
\text { chloro- } \\
\text { benzene } \\
(\mu \mathrm{g} / \mathrm{L}) \\
(34571)\end{array}$ & $\begin{array}{c}\text { Carbon tetrachloride } \\
\text { (tetrachloro- } \\
\text { methane) } \\
(\mu \mathrm{g} / \mathrm{L}) \\
(32102) \\
\end{array}$ & $\begin{array}{c}\text { Chloro- } \\
\text { benzene } \\
(\mu \mathrm{g} / \mathrm{L}) \\
(34301)\end{array}$ \\
\hline Benchmark type & MCL-US & MCL-US & MCL-US & MCL-US & MCL-US & MCL-US & MCL-US & MCL-CA & HAL-US & MCL-CA & MCL-CA & MCL-CA \\
\hline Benchmark level & ${ }^{2} 80$ & 280 & 280 & 280 & 5 & 5 & 200 & 5 & 600 & 5 & 0.5 & 70 \\
\hline [LRL] & {$[0.03]$} & {$[0.034]$} & {$[0.12]$} & {$[0.1]$} & {$[0.026]$} & {$[0.022]$} & {$[0.030]$} & {$[0.08]$} & {$[0.024]$} & {$[0.026]$} & {$[0.06]$} & {$[0.026]$} \\
\hline \multicolumn{13}{|c|}{ Peninsular Ranges study area (36 grid wells sampled) } \\
\hline HR-PR-01 & 0.08 & - & - & - & - & - & - & - & - & - & - & - \\
\hline HR-PR-02 & 0.03 & - & - & - & ${ }^{5} 0.012$ & - & - & - & - & - & - & - \\
\hline HR-PR-07 & 0.02 & - & - & - & - & - & - & - & - & - & - & - \\
\hline HR-PR-08 & - & - & - & - & 0.058 & - & - & - & - & - & - & - \\
\hline HR-PR-12 & 1.4 & - & - & - & - & - & - & - & - & - & - & - \\
\hline HR-PR-13 & 0.06 & - & - & - & - & - & - & - & - & - & - & - \\
\hline HR-PR-15 & 0.11 & - & - & - & - & - & - & - & - & - & 0.03 & - \\
\hline HR-PR-17 & 0.12 & - & - & - & ${ }^{5} 0.012$ & - & - & - & - & - & - & - \\
\hline HR-PR-18 & - & - & - & - & - & - & - & - & - & - & - & - \\
\hline HR-PR-20 & 0.14 & - & - & - & - & - & - & - & - & - & - & - \\
\hline HR-PR-22 & - & - & - & - & - & - & - & - & - & - & - & - \\
\hline HR-PR-24 & - & - & - & - & - & - & - & - & - & - & - & - \\
\hline HR-PR-25 & - & - & - & - & - & - & - & - & - & - & - & - \\
\hline HR-PR-28 & - & - & - & - & - & - & - & - & - & - & - & - \\
\hline HR-PR-30 & 0.12 & - & - & - & - & - & - & - & - & - & - & - \\
\hline HR-PR-31 & 0.10 & - & - & - & - & - & - & - & - & - & - & - \\
\hline HR-PR-33 & 0.02 & - & - & - & - & - & - & - & - & - & - & - \\
\hline HR-PR-35 & 1.4 & - & - & - & - & - & - & - & - & - & - & - \\
\hline
\end{tabular}


Table 5. Volatile organic compounds (VOCs) detected in samples collected for the Santa Cruz, San Gabriel, and Peninsular Ranges Hard Rock Aquifers (Hard Rock) study unit, March 2011 through March 2012, California GAMA Priority Basin Project.-Continued

[The five-digit U.S. Geological Survey (USGS) parameter code below the constituent name is used to uniquely identify a specific constituent or property. Samples from 110 wells were analyzed, but only samples with detections of at least one constituent are listed. Constituents are grouped by primary use or source and listed in order of decreasing detection frequency for each group in the 94 grid wells sampled. All analytes are listed in table 3A. GAMA well identification number: HR-SC, Santa Cruz study area grid well; HR-SG, San Gabriel study area grid well; HR-SGU, San Gabriel study area understanding well; HR-PR, Peninsular Ranges study area grid well; HR-PRFP, Peninsular Ranges study area flow-path well; HR-PRU, Peninsular Ranges study area understanding well. Reporting levels, benchmark types, and benchmark levels as of July 6, 2012. Benchmark type: Maximum contaminant level benchmarks are listed as MCL-US when the MCL-US and MCL-CA are identical, and as MCL-CA when the MCL-CA is lower than the MCL-US or no MCL-US exists. MCL-US, U.S. Environmental Protection Agency maximum contaminant level; MCL-CA, California Department of Public Health maximum contaminant level; NL-CA, CDPH notification level. Other abbreviations: E, estimated or having a higher degree of uncertainty; LRL, laboratory reporting level; $\mu \mathrm{g} / \mathrm{L}$, micrograms per liter; na, not available; —, not detected]

\begin{tabular}{|c|c|c|c|c|c|c|c|c|c|c|c|c|}
\hline \multirow[b]{2}{*}{$\begin{array}{c}\text { GAMA } \\
\text { well } \\
\text { identification } \\
\text { number }\end{array}$} & \multicolumn{4}{|c|}{ Disinfection byproducts } & \multicolumn{8}{|c|}{ Solvents } \\
\hline & $\begin{array}{l}\text { Chloroform } \\
\text { (trichloro- } \\
\text { methane) } \\
(\mu \mathrm{g} / \mathrm{L}) \\
(32106)\end{array}$ & $\begin{array}{l}\text { Bromo- } \\
\text { dichloro- } \\
\text { methane } \\
(\mu \mathrm{g} / \mathrm{L}) \\
(32101)\end{array}$ & $\begin{array}{l}\text { Dibromo- } \\
\text { chloro- } \\
\text { methane } \\
(\mu \mathrm{g} / \mathrm{L}) \\
(32105)\end{array}$ & $\begin{array}{c}\text { Bromoform } \\
\text { (tribromo- } \\
\text { methane) } \\
(\mu \mathrm{g} / \mathrm{L}) \\
(32104)\end{array}$ & $\begin{array}{c}\text { Tetra- } \\
\text { chloro- } \\
\text { ethene } \\
(\mu \mathrm{g} / \mathrm{L}) \\
(34475)\end{array}$ & $\begin{array}{c}\text { Trichloro- } \\
\text { ethene } \\
(\mu \mathrm{g} / \mathrm{L}) \\
(39180)\end{array}$ & $\begin{array}{c}\text { 1,1,1-Tri- } \\
\text { chloro- } \\
\text { ethane } \\
(\mu \mathrm{g} / \mathrm{L}) \\
(34506)\end{array}$ & $\begin{array}{l}\text { 1,2,4-Tri- } \\
\text { chloro- } \\
\text { benzene } \\
(\mu \mathrm{g} / \mathrm{L}) \\
(34551)\end{array}$ & $\begin{array}{c}\text { 1,3-Di- } \\
\text { chloro- } \\
\text { benzene } \\
(\mu \mathrm{g} / \mathrm{L}) \\
(34566)\end{array}$ & $\begin{array}{c}\text { 1,4-Di- } \\
\text { chloro- } \\
\text { benzene } \\
(\mu \mathrm{g} / \mathrm{L}) \\
(34571)\end{array}$ & $\begin{array}{c}\text { Carbon tetrachloride } \\
\text { (tetrachloro- } \\
\text { methane) } \\
(\mu \mathrm{g} / \mathrm{L}) \\
(32102)\end{array}$ & $\begin{array}{c}\text { Chloro- } \\
\text { benzene } \\
(\mu \mathrm{g} / \mathrm{L}) \\
(34301)\end{array}$ \\
\hline Benchmark type & MCL-US & MCL-US & MCL-US & MCL-US & MCL-US & MCL-US & MCL-US & MCL-CA & HAL-US & MCL-CA & MCL-CA & MCL-CA \\
\hline Benchmark level & ${ }^{2} 80$ & ${ }^{2} 80$ & ${ }^{2} 80$ & ${ }^{2} 80$ & 5 & 5 & 200 & 5 & 600 & 5 & 0.5 & 70 \\
\hline [LRL] & {$[0.03]$} & {$[0.034]$} & {$[0.12]$} & {$[0.1]$} & {$[0.026]$} & {$[0.022]$} & {$[0.030]$} & {$[0.08]$} & {$[0.024]$} & {$[0.026]$} & {$[0.06]$} & {$[0.026]$} \\
\hline \multicolumn{13}{|c|}{ Peninsular Ranges study area (36 grid wells sampled) —Continued } \\
\hline $\begin{array}{l}\text { Number of wells with } \\
\text { detections }\end{array}$ & 12 & 0 & 0 & 0 & 3 & 0 & 0 & 0 & 0 & 0 & 1 & 0 \\
\hline Detection frequency (percent) & 33 & 0 & 0 & 0 & 8.3 & 0 & 0 & 0 & 0 & 0 & 2.8 & 0 \\
\hline \multicolumn{13}{|c|}{ Hard Rock understanding wells (16 wells sampled) ${ }^{6}$} \\
\hline HR-PRFP-02 & 1.1 & 0.173 & - & - & - & - & - & - & - & - & - & - \\
\hline HR-PRFP-08 & - & - & - & - & - & - & - & - & - & - & - & - \\
\hline HR-PRFP-09 & 0.06 & - & - & - & - & - & - & - & - & - & - & - \\
\hline HR-PRFP-11 & 0.10 & - & - & - & 0.022 & - & - & - & - & - & - & - \\
\hline HR-PRFP-12 & - & - & - & - & ${ }^{5} 0.011$ & - & - & - & - & - & - & - \\
\hline HR-PRFP-13 & - & - & - & - & - & - & - & - & - & - & - & - \\
\hline HR-PRU-01 & 0.02 & - & - & - & - & - & - & - & - & - & - & - \\
\hline
\end{tabular}


Table 5. Volatile organic compounds (VOCs) detected in samples collected for the Santa Cruz, San Gabriel, and Peninsular Ranges Hard Rock Aquifers (Hard Rock) study unit, March 2011 through March 2012, California GAMA Priority Basin Project.—Continued

[The five-digit U.S. Geological Survey (USGS) parameter code below the constituent name is used to uniquely identify a specific constituent or property. Samples from 110 wells were analyzed, but only samples with detections of at least one constituent are listed. Constituents are grouped by primary use or source and listed in order of decreasing detection frequency for each group in the 94 grid wells sampled. All analytes are listed in table 3A. GAMA well identification number: HR-SC, Santa Cruz study area grid well; HR-SG, San Gabriel study area grid well; HR-SGU, San Gabriel study area understanding well; HR-PR, Peninsular Ranges study area grid well; HR-PRFP, Peninsular Ranges study area flow-path well; HR-PRU, Peninsular Ranges study area understanding well. Reporting levels, benchmark types, and benchmark levels as of July 6, 2012. Benchmark type: Maximum contaminant level benchmarks are listed as MCL-US when the MCL-US and MCL-CA are identical, and as MCL-CA when the MCL-CA is lower than the MCL-US or no MCL-US exists. MCL-US, U.S. Environmental Protection Agency maximum contaminant level; MCL-CA, California Department of Public Health maximum contaminant level; NL-CA, CDPH notification level. Other abbreviations: E, estimated or having a higher degree of uncertainty; LRL, laboratory reporting level; $\mu \mathrm{g} / \mathrm{L}$, micrograms per liter; na, not available; - , not detected]

\begin{tabular}{|c|c|c|c|c|c|c|c|c|c|}
\hline \multirow{2}{*}{$\begin{array}{c}\text { GAMA well } \\
\text { identification } \\
\text { number }\end{array}$} & \multicolumn{2}{|c|}{ Organic synthesis } & \multirow{2}{*}{$\begin{array}{c}\text { Gasoline oxygenate } \\
\text { Methyl tert-butyl ether } \\
\text { (MTBE) } \\
(\mu \mathrm{g} / \mathrm{L}) \\
(78032) \\
\end{array}$} & \multirow{2}{*}{$\begin{array}{c}\text { Organic } \\
\text { synthesis or } \\
\text { natural } \\
\text { Carbon } \\
\text { disulfide } \\
(\mu \mathrm{g} / \mathrm{L}) \\
(77041)\end{array}$} & \multicolumn{2}{|c|}{ Refrigerants } & \multirow{2}{*}{$\begin{array}{c}\text { Fire retardant } \\
\text { Bromochloro- } \\
\text { methane } \\
(\mu \mathrm{g} / \mathrm{L}) \\
(77297) \\
\end{array}$} & \multirow{2}{*}{$\begin{array}{c}\text { Detections } \\
\text { per well }\end{array}$} & \multirow{2}{*}{$\begin{array}{c}\text { VOC } \\
\text { detection } \\
\text { summary }^{1}\end{array}$} \\
\hline & $\begin{array}{c}\text { 1,1-Dichloro- } \\
\text { ethene } \\
\text { ( } \mu \mathrm{g} / \mathrm{L}) \\
(34501)\end{array}$ & $\begin{array}{l}\text { 1,2,3-Trichloro- } \\
\text { benzene } \\
(\mu \mathrm{g} / \mathrm{L}) \\
(77613)\end{array}$ & & & $\begin{array}{c}\text { Trichlorotrifluoroethane } \\
\text { (CFC-113) } \\
(\mu \mathrm{g} / \mathrm{L}) \\
(77652)\end{array}$ & $\begin{array}{c}\text { Trichlorofluoromethane } \\
\text { (CFC-11) } \\
(\mu \mathrm{g} / \mathrm{L}) \\
(34488)\end{array}$ & & & \\
\hline $\begin{array}{l}\text { Benchmark } \\
\text { type }\end{array}$ & MCL-CA & na & MCL-CA & NL-CA & MCL-CA & MCL-CA & HAL-US & & \\
\hline $\begin{array}{l}\text { Benchmark } \\
\text { level }\end{array}$ & 6 & na & 13 & 160 & 1,200 & 150 & 90 & & \\
\hline [LRL] & {$[0.022]$} & {$[0.06]$} & {$[0.1]$} & ${ }^{3}[0.1]$ & {$[0.034]$} & {$[0.06]$} & {$[0.06]$} & & \\
\hline \multicolumn{10}{|c|}{ Hard Rock study unit (94 grid wells sampled) } \\
\hline $\begin{array}{l}\text { Number of } \\
\text { wells with } \\
\text { detections }\end{array}$ & 7 & 1 & 7 & 2 & 2 & 1 & 1 & & 41 \\
\hline $\begin{array}{l}\text { Detection } \\
\text { frequency } \\
\text { (percent) }\end{array}$ & 7.4 & 1.1 & 7.4 & 2.1 & 2.1 & 1.1 & 1.1 & & 44 \\
\hline \multicolumn{10}{|c|}{ Santa Cruz study area ( 25 grid wells sampled) } \\
\hline HR-SC-02 & - & - & - & - & - & - & - & 1 & \\
\hline HR-SC-04 & 0.031 & - & - & - & - & - & - & 2 & \\
\hline HR-SC-08 & - & - & - & - & - & - & - & 1 & \\
\hline HR-SC-11 & - & - & - & - & - & - & - & 1 & \\
\hline HR-SC-13 & 0.034 & - & - & - & - & - & - & 1 & \\
\hline HR-SC-14 & - & - & - & - & - & - & - & 1 & \\
\hline HR-SC-16 ${ }^{4}$ & - & - & - & - & - & - & ${ }^{5} 0.02$ & 5 & \\
\hline HR-SC-17 & - & - & 0.05 & - & - & - & - & 3 & \\
\hline HR-SC-20 & ${ }^{5} 0.010$ & - & - & - & - & - & - & 3 & \\
\hline HR-SC-21 & 0.030 & - & - & - & - & - & - & 1 & \\
\hline
\end{tabular}


Table 5. Volatile organic compounds (VOCs) detected in samples collected for the Santa Cruz, San Gabriel, and Peninsular Ranges Hard Rock Aquifers (Hard Rock) study unit, March 2011 through March 2012, California GAMA Priority Basin Project.-Continued

[The five-digit U.S. Geological Survey (USGS) parameter code below the constituent name is used to uniquely identify a specific constituent or property. Samples from 110 wells were analyzed, but only samples with detections of at least one constituent are listed. Constituents are grouped by primary use or source and listed in order of decreasing detection frequency for each group in the 94 grid wells sampled. All analytes are listed in table 3A. GAMA well identification number: HR-SC, Santa Cruz study area grid well; HR-SG, San Gabriel study area grid well; HR-SGU, San Gabriel study area understanding well; HR-PR, Peninsular Ranges study area grid well; HR-PRFP, Peninsular Ranges study area flow-path well; HR-PRU, Peninsular Ranges study area understanding well. Reporting levels, benchmark types, and benchmark levels as of July 6, 2012. Benchmark type: Maximum contaminant level benchmarks are listed as MCL-US when the MCL-US and MCL-CA are identical, and as MCL-CA when the MCL-CA is lower than the MCL-US or no MCL-US exists. MCL-US, U.S. Environmental Protection Agency maximum contaminant level; MCL-CA, California Department of Public Health maximum contaminant level; NL-CA, CDPH notification level. Other abbreviations: E, estimated or having a higher degree of uncertainty; LRL, laboratory reporting level; $\mu \mathrm{g} / \mathrm{L}$, micrograms per liter; na, not available; - , not detected]

\begin{tabular}{|c|c|c|c|c|c|c|c|c|c|}
\hline \multirow{2}{*}{$\begin{array}{c}\text { GAMA well } \\
\text { identification } \\
\text { number }\end{array}$} & \multicolumn{2}{|c|}{ Organic synthesis } & \multirow{2}{*}{$\begin{array}{l}\text { Gasoline oxygenate } \\
\begin{array}{c}\text { Methyl tert-butyl ether } \\
\text { (MTBE) } \\
(\mu \mathrm{g} / \mathrm{L}) \\
(78032)\end{array}\end{array}$} & \multirow{2}{*}{$\begin{array}{c}\text { Organic } \\
\text { synthesis or } \\
\text { natural } \\
\text { Carbon } \\
\text { disulfide } \\
(\mu \mathrm{g} / \mathrm{L}) \\
(77041) \\
\end{array}$} & \multicolumn{2}{|c|}{ Refrigerants } & \multirow{2}{*}{$\begin{array}{c}\text { Fire retardant } \\
\text { Bromochloro- } \\
\text { methane } \\
(\mu \mathrm{g} / \mathrm{L}) \\
(77297)\end{array}$} & \multirow{2}{*}{$\begin{array}{l}\text { Detections } \\
\text { per well }\end{array}$} & \multirow{2}{*}{$\begin{array}{c}\text { VOC } \\
\text { detection } \\
\text { summary }{ }^{1}\end{array}$} \\
\hline & $\begin{array}{l}\text { 1,1-Dichloro- } \\
\text { ethene } \\
(\mu \mathrm{g} / \mathrm{L}) \\
(34501)\end{array}$ & $\begin{array}{l}\text { 1,2,3-Trichloro- } \\
\text { benzene } \\
(\mu \mathrm{g} / \mathrm{L}) \\
(77613)\end{array}$ & & & $\begin{array}{c}\text { Trichlorotrifluoroethane } \\
\text { (CFC-113) } \\
(\mu \mathrm{g} / \mathrm{L}) \\
(77652)\end{array}$ & $\begin{array}{c}\text { Trichlorofluoromethane } \\
\text { (CFC-11) } \\
(\mu \mathrm{g} / \mathrm{L}) \\
(34488)\end{array}$ & & & \\
\hline $\begin{array}{l}\text { Benchmark } \\
\text { type }\end{array}$ & MCL-CA & na & MCL-CA & NL-CA & MCL-CA & MCL-CA & HAL-US & & \\
\hline $\begin{array}{l}\text { Benchmark } \\
\text { level }\end{array}$ & 6 & na & 13 & 160 & 1,200 & 150 & 90 & & \\
\hline [LRL] & [0.022] & {$[0.06]$} & [0.1] & ${ }^{3}[0.1]$ & [0.034] & {$[0.06]$} & {$[0.06]$} & & \\
\hline \multicolumn{10}{|c|}{ Santa Cruz study area ( 25 grid wells sampled)—Continued } \\
\hline HR-SC-22 & 0.019 & - & - & - & - & - & - & 1 & \\
\hline HR-SC-23 & 0.019 & - & - & - & - & - & - & 1 & \\
\hline HR-SC-24 & - & - & - & - & 0.021 & - & - & 2 & \\
\hline HR-SC-25 & - & - & 0.06 & - & - & - & - & 5 & \\
\hline $\begin{array}{l}\text { Number of } \\
\text { wells with } \\
\text { detections }\end{array}$ & 6 & 0 & 2 & 0 & 1 & 0 & 1 & & 14 \\
\hline $\begin{array}{l}\text { Detection } \\
\text { frequency } \\
\text { (percent) }\end{array}$ & 24 & 0 & 8.0 & 0 & 4.0 & 0 & 4.0 & & 56 \\
\hline \multicolumn{10}{|c|}{ San Gabriel study area (33 grid wells sampled) } \\
\hline HR-SG-02 & - & - & - & - & - & - & - & 1 & \\
\hline HR-SG-04 & 0.035 & - & - & - & 0.1 & 0.06 & - & 5 & \\
\hline HR-SG-05 & - & - & - & - & - & - & - & 1 & \\
\hline HR-SG-08 & - & - & - & - & - & - & - & 3 & \\
\hline HR-SG-15 & - & - & - & - & - & - & - & 4 & \\
\hline HR-SG-27 & - & 1.24 & - & - & - & - & - & 7 & \\
\hline HR-SG-28 & - & - & - & - & - & - & - & 1 & \\
\hline HR-SG-31 & - & - & - & - & - & - & - & 1 & \\
\hline HR-SG-33 & - & - & - & - & - & - & - & 1 & \\
\hline
\end{tabular}


Table 5. Volatile organic compounds (VOCs) detected in samples collected for the Santa Cruz, San Gabriel, and Peninsular Ranges Hard Rock Aquifers (Hard Rock) study unit, March 2011 through March 2012, California GAMA Priority Basin Project.-Continued

[The five-digit U.S. Geological Survey (USGS) parameter code below the constituent name is used to uniquely identify a specific constituent or property. Samples from 110 wells were analyzed, but only samples with detections of at least one constituent are listed. Constituents are grouped by primary use or source and listed in order of decreasing detection frequency for each group in the 94 grid wells sampled. All analytes are listed in table 3A. GAMA well identification number: HR-SC, Santa Cruz study area grid well; HR-SG, San Gabriel study area grid well; HR-SGU, San Gabriel study area understanding well; HR-PR, Peninsular Ranges study area grid well; HR-PRFP, Peninsular Ranges study area flow-path well; HR-PRU, Peninsular Ranges study area understanding well. Reporting levels, benchmark types, and benchmark levels as of July 6, 2012. Benchmark type: Maximum contaminant level benchmarks are listed as MCL-US when the MCL-US and MCL-CA are identical, and as MCL-CA when the MCL-CA is lower than the MCL-US or no MCL-US exists. MCL-US, U.S. Environmental Protection Agency maximum contaminant level; MCL-CA, California Department of Public Health maximum contaminant level; NL-CA, CDPH notification level. Other abbreviations: E, estimated or having a higher degree of uncertainty; LRL, laboratory reporting level; $\mu \mathrm{g} / \mathrm{L}$, micrograms per liter; na, not available; -, not detected]

\begin{tabular}{|c|c|c|c|c|c|c|c|c|c|}
\hline \multirow{2}{*}{$\begin{array}{c}\text { GAMA well } \\
\text { identification } \\
\text { number }\end{array}$} & \multicolumn{2}{|c|}{ Organic synthesis } & \multirow{2}{*}{$\begin{array}{c}\text { Gasoline oxygenate } \\
\text { Methyl tert-butyl ether } \\
\text { (MTBE) } \\
(\mu \mathrm{g} / \mathrm{L}) \\
(78032) \\
\end{array}$} & \multirow{2}{*}{$\begin{array}{c}\text { Organic } \\
\text { synthesis or } \\
\text { natural } \\
\text { Carbon } \\
\text { disulfide } \\
(\mu \mathrm{g} / \mathrm{L}) \\
(77041)\end{array}$} & \multicolumn{2}{|c|}{ Refrigerants } & \multirow{2}{*}{$\begin{array}{c}\text { Fire retardant } \\
\text { Bromochloro- } \\
\text { methane } \\
(\mu \mathrm{g} / \mathrm{L}) \\
(77297) \\
\end{array}$} & \multirow{2}{*}{$\begin{array}{c}\text { Detections } \\
\text { per well }\end{array}$} & \multirow{2}{*}{$\begin{array}{c}\text { VOC } \\
\text { detection } \\
\text { summary }{ }^{1}\end{array}$} \\
\hline & $\begin{array}{l}\text { 1,1-Dichloro- } \\
\text { ethene } \\
(\mu \mathrm{g} / \mathrm{L}) \\
(34501)\end{array}$ & $\begin{array}{l}\text { 1,2,3-Trichloro- } \\
\text { benzene } \\
(\mu \mathrm{g} / \mathrm{L}) \\
(77613)\end{array}$ & & & $\begin{array}{c}\text { Trichlorotrifluoroethane } \\
\text { (CFC-113) } \\
(\mu \mathrm{g} / \mathrm{L}) \\
(77652)\end{array}$ & $\begin{array}{c}\text { Trichlorofluoromethane } \\
\text { (CFC-11) } \\
(\mu \mathrm{g} / \mathrm{L}) \\
(\mathbf{3 4 4 8 8 )}\end{array}$ & & & \\
\hline $\begin{array}{l}\text { Benchmark } \\
\text { type }\end{array}$ & MCL-CA & na & MCL-CA & NL-CA & MCL-CA & MCL-CA & HAL-US & & \\
\hline $\begin{array}{l}\text { Benchmark } \\
\text { level }\end{array}$ & 6 & na & 13 & 160 & 1,200 & 150 & 90 & & \\
\hline [LRL] & {$[0.022]$} & {$[0.06]$} & {$[0.1]$} & ${ }^{3}[0.1]$ & {$[0.034]$} & {$[0.06]$} & {$[0.06]$} & & \\
\hline \multicolumn{10}{|c|}{ San Gabriel study area (33 grid wells sampled)_Continued } \\
\hline $\begin{array}{l}\text { Number of } \\
\text { wells with } \\
\text { detections }\end{array}$ & 1 & 1 & 0 & 0 & 1 & 1 & & & 9 \\
\hline $\begin{array}{l}\text { Detection } \\
\text { frequency } \\
\text { (percent) }\end{array}$ & 3.0 & 3.0 & 0 & 0 & 3.0 & 3.0 & & & 27 \\
\hline \multicolumn{10}{|c|}{ Peninsular Ranges study area (36 grid wells sampled) } \\
\hline HR-PR-01 & - & - & - & - & - & - & - & 1 & \\
\hline HR-PR-02 & - & - & - & - & - & - & - & 2 & \\
\hline HR-PR-07 & - & - & - & - & - & - & - & 1 & \\
\hline HR-PR-08 & - & - & - & - & - & - & - & 1 & \\
\hline HR-PR-12 & - & - & - & - & - & - & - & 1 & \\
\hline HR-PR-13 & - & - & - & - & - & - & - & 1 & \\
\hline HR-PR-15 & - & - & - & - & - & - & - & 2 & \\
\hline HR-PR-17 & - & - & - & - & - & - & - & 2 & \\
\hline HR-PR-18 & - & - & 0.07 & - & - & - & - & 1 & \\
\hline HR-PR-20 & - & - & - & - & - & - & - & 1 & \\
\hline
\end{tabular}


Table 5. Volatile organic compounds (VOCs) detected in samples collected for the Santa Cruz, San Gabriel, and Peninsular Ranges Hard Rock Aquifers (Hard Rock) study unit, March 2011 through March 2012, California GAMA Priority Basin Project.-Continued

[The five-digit U.S. Geological Survey (USGS) parameter code below the constituent name is used to uniquely identify a specific constituent or property. Samples from 110 wells were analyzed, but only samples with detections of at least one constituent are listed. Constituents are grouped by primary use or source and listed in order of decreasing detection frequency for each group in the 94 grid wells sampled. All analytes are listed in table 3A. GAMA well identification number: HR-SC, Santa Cruz study area grid well; HR-SG, San Gabriel study area grid well; HR-SGU, San Gabriel study area understanding well; HR-PR, Peninsular Ranges study area grid well; HR-PRFP, Peninsular Ranges study area flow-path well; HR-PRU, Peninsular Ranges study area understanding well. Reporting levels, benchmark types, and benchmark levels as of July 6, 2012. Benchmark type: Maximum contaminant level benchmarks are listed as MCL-US when the MCL-US and MCL-CA are identical, and as MCL-CA when the MCL-CA is lower than the MCL-US or no MCL-US exists. MCL-US, U.S. Environmental Protection Agency maximum contaminant level; MCL-CA, California Department of Public Health maximum contaminant level; NL-CA, CDPH notification level. Other abbreviations: E, estimated or having a higher degree of uncertainty; LRL, laboratory reporting level; $\mu \mathrm{g} / \mathrm{L}$, micrograms per liter; na, not available; - , not detected]

\begin{tabular}{|c|c|c|c|c|c|c|c|c|c|}
\hline \multirow{2}{*}{$\begin{array}{l}\text { GAMA well } \\
\text { identification } \\
\text { number }\end{array}$} & \multicolumn{2}{|c|}{ Organic synthesis } & \multirow{2}{*}{$\begin{array}{c}\text { Gasoline oxygenate } \\
\text { Methyl tert-butyl ether } \\
\text { (MTBE) } \\
(\mu \mathrm{g} / \mathrm{L}) \\
(78032) \\
\end{array}$} & \multirow{2}{*}{$\begin{array}{c}\text { Organic } \\
\text { synthesis or } \\
\text { natural } \\
\text { Carbon } \\
\text { disulfide } \\
\text { ( } \mu \mathrm{g} / \mathrm{L}) \\
(77041)\end{array}$} & \multicolumn{2}{|c|}{ Refrigerants } & \multirow{2}{*}{$\begin{array}{c}\text { Fire retardant } \\
\text { Bromochloro- } \\
\text { methane } \\
(\mu \mathrm{g} / \mathrm{L}) \\
(77297)\end{array}$} & \multirow{2}{*}{$\begin{array}{c}\text { Detections } \\
\text { per well }\end{array}$} & \multirow{2}{*}{$\begin{array}{c}\text { VOC } \\
\text { detection } \\
\text { summary }{ }^{1}\end{array}$} \\
\hline & $\begin{array}{c}\text { 1,1-Dichloro- } \\
\text { ethene } \\
(\mu \mathrm{g} / \mathrm{L}) \\
(34501)\end{array}$ & $\begin{array}{l}\text { 1,2,3-Trichloro- } \\
\text { benzene } \\
(\mu \mathrm{g} / \mathrm{L}) \\
(77613)\end{array}$ & & & $\begin{array}{l}\text { Trichlorotrifluoroethane } \\
\text { (CFC-113) } \\
(\mu \mathrm{g} / \mathrm{L}) \\
(77652)\end{array}$ & $\begin{array}{c}\text { Trichlorofluoromethane } \\
\text { (CFC-11) } \\
(\mu \mathrm{g} / \mathrm{L}) \\
(34488)\end{array}$ & & & \\
\hline $\begin{array}{l}\text { Benchmark } \\
\text { type }\end{array}$ & MCL-CA & na & MCL-CA & NL-CA & MCL-CA & MCL-CA & HAL-US & & \\
\hline $\begin{array}{l}\text { Benchmark } \\
\text { level }\end{array}$ & 6 & na & 13 & 160 & 1,200 & 150 & 90 & & \\
\hline [LRL] & {$[0.022]$} & {$[0.06]$} & {$[0.1]$} & ${ }^{3}[0.1]$ & {$[0.034]$} & {$[0.06]$} & {$[0.06]$} & & \\
\hline \multicolumn{10}{|c|}{ Peninsular Ranges study area (36 grid wells sampled) —Continued } \\
\hline HR-PR-22 & - & - & ${ }^{5} 0.02$ & E0.05 & - & - & - & 2 & \\
\hline HR-PR-24 & - & - & 0.14 & - & - & - & - & 1 & \\
\hline HR-PR-25 & - & - & ${ }^{5} 0.03$ & - & - & - & - & 1 & \\
\hline HR-PR-28 & - & - & 0.53 & E0.13 & - & - & - & 2 & \\
\hline HR-PR-30 & - & - & - & - & - & - & - & 1 & \\
\hline HR-PR-31 & - & - & - & - & - & - & - & 1 & \\
\hline HR-PR-33 & - & - & - & - & - & - & - & 1 & \\
\hline HR-PR-35 & - & - & - & - & - & - & - & 1 & \\
\hline $\begin{array}{l}\text { Number of } \\
\text { wells with } \\
\text { detections }\end{array}$ & 0 & 0 & 5 & 2 & 0 & 0 & & & 18 \\
\hline $\begin{array}{l}\text { Detection } \\
\text { frequency } \\
\text { (percent) }\end{array}$ & 0 & 0 & 14 & 5.6 & 0 & 0 & & & 50 \\
\hline
\end{tabular}


Table 5. Volatile organic compounds (VOCs) detected in samples collected for the Santa Cruz, San Gabriel, and Peninsular Ranges Hard Rock Aquifers (Hard Rock) study unit, March 2011 through March 2012, California GAMA Priority Basin Project.—Continued

[The five-digit U.S. Geological Survey (USGS) parameter code below the constituent name is used to uniquely identify a specific constituent or property. Samples from 110 wells were analyzed, but only samples with detections of at least one constituent are listed. Constituents are grouped by primary use or source and listed in order of decreasing detection frequency for each group in the 94 grid wells sampled. All analytes are listed in table 3A. GAMA well identification number: HR-SC, Santa Cruz study area grid well; HR-SG, San Gabriel study area grid well; HR-SGU, San Gabriel study area understanding well; HR-PR, Peninsular Ranges study area grid well; HR-PRFP, Peninsular Ranges study area flow-path well; HR-PRU, Peninsular Ranges study area understanding well. Reporting levels, benchmark types, and benchmark levels as of July 6, 2012. Benchmark type: Maximum contaminant level benchmarks are listed as MCL-US when the MCL-US and MCL-CA are identical, and as MCL-CA when the MCL-CA is lower than the MCL-US or no MCL-US exists. MCL-US, U.S. Environmental Protection Agency maximum contaminant level; MCL-CA, California Department of Public Health maximum contaminant level; NL-CA, CDPH notification level. Other abbreviations: E, estimated or having a higher degree of uncertainty; LRL, laboratory reporting level; $\mu \mathrm{g} / \mathrm{L}$, micrograms per liter; na, not available; - , not detected]

\begin{tabular}{|c|c|c|c|c|c|c|c|c|c|}
\hline \multirow{2}{*}{$\begin{array}{l}\text { GAMA well } \\
\text { identification } \\
\text { number }\end{array}$} & \multicolumn{2}{|c|}{ Organic synthesis } & \multirow{2}{*}{$\begin{array}{c}\text { Gasoline oxygenate } \\
\text { Methyl tert-butyl ether } \\
\text { (MTBE) } \\
(\mu \mathrm{g} / \mathrm{L}) \\
(78032)\end{array}$} & \multirow{2}{*}{$\begin{array}{c}\text { Organic } \\
\text { synthesis or } \\
\text { natural } \\
\text { Carbon } \\
\text { disulfide } \\
(\mu \mathrm{g} / \mathrm{L}) \\
(77041)\end{array}$} & \multicolumn{2}{|c|}{ Refrigerants } & \multirow{2}{*}{$\begin{array}{c}\text { Fire retardant } \\
\text { Bromochloro- } \\
\text { methane } \\
(\mu \mathrm{g} / \mathrm{L}) \\
(77297)\end{array}$} & \multirow{2}{*}{$\begin{array}{c}\text { Detections } \\
\text { per well }\end{array}$} & \multirow{2}{*}{$\begin{array}{c}\text { VOC } \\
\text { detection } \\
\text { summary }{ }^{1}\end{array}$} \\
\hline & $\begin{array}{c}\text { 1,1-Dichloro- } \\
\text { ethene } \\
(\mu \mathrm{g} / \mathrm{L}) \\
(34501) \\
\end{array}$ & $\begin{array}{c}1,2,3-\text { Trichloro- } \\
\text { benzene } \\
(\mu \mathrm{g} / \mathrm{L}) \\
(77613) \\
\end{array}$ & & & $\begin{array}{c}\text { Trichlorotrifluoroethane } \\
\text { (CFC-113) } \\
(\mu \mathrm{g} / \mathrm{L}) \\
(77652) \\
\end{array}$ & $\begin{array}{c}\text { Trichlorofluoromethane } \\
\text { (CFC-11) } \\
(\mu \mathrm{g} / \mathrm{L}) \\
(\mathbf{3 4 4 8 8 )}\end{array}$ & & & \\
\hline $\begin{array}{l}\text { Benchmark } \\
\text { type }\end{array}$ & MCL-CA & na & MCL-CA & NL-CA & MCL-CA & MCL-CA & HAL-US & & \\
\hline $\begin{array}{l}\text { Benchmark } \\
\text { level }\end{array}$ & 6 & na & 13 & 160 & 1,200 & 150 & 90 & & \\
\hline [LRL] & {$[0.022]$} & {$[0.06]$} & {$[0.1]$} & ${ }^{3}[0.1]$ & {$[0.034]$} & {$[0.06]$} & {$[0.06]$} & & \\
\hline \multicolumn{10}{|c|}{ Hard Rock understanding wells (16 wells sampled) ${ }^{6}$} \\
\hline HR-PRFP-02 & - & - & - & - & - & - & - & 2 & \\
\hline HR-PRFP-08 & - & - & - & E0.08 & - & - & - & 1 & \\
\hline HR-PRFP-09 & - & - & 0.07 & - & - & - & - & 2 & \\
\hline HR-PRFP-11 & - & - & - & - & - & - & - & 2 & \\
\hline HR-PRFP-12 & - & - & - & - & - & - & - & 1 & \\
\hline HR-PRFP-13 & - & - & - & - & - & 0.04 & - & 1 & \\
\hline HR-PRU-01 & - & - & - & - & - & - & - & 1 & \\
\hline
\end{tabular}

${ }^{1}$ Study reporting levels (SRLs) determined on the basis of the quality-control data collected between May 2004 and September 2010 for the first 32 study units of the California GAMA Priority Basin Project (Fram and others, 2012) were applied to three VOCs. Detections of 1,2,4-trimethylbenzene, carbon disulfide, and toluene at concentrations less than the respective SRL were reclassified as nondetections and reported with the following comment in the USGS National Water Information System (NWIS) database: Result is $<$ or = reported value, based on quality-control data (see Fram and others, 2012). SRLs could not be determined for three other VOCs, and instead all detections for acetone, 2-butanone, and tetrahydrofuran were censored from this dataset and coded in the USGS database as "reviewed and rejected." See appendix table A3 for more information.

${ }^{2}$ The MCL-US benchmark for trihalomethanes is the sum of chloroform, bromodichloromethane, dibromochloromethane, and bromoform.

${ }^{3}$ The highest LRL for carbon disulfide during March 2011 through March 2012 was greater than the established SRL of $0.03 \mu \mathrm{g} / \mathrm{L}$ and therefore is used in this table.

${ }^{4}$ The HR-SC-16 groundwater well pumps to a nearby storage tank that is sometimes chlorinated.

${ }^{5}$ Result is below long-term method detection level.

${ }^{6}$ Understanding wells were not included in statistical calculations. Due to high concentrations of known contaminants 2-butanone, acetone, and tetrahydrofuran (31.0, 19.8, and 112 $\mu \mathrm{g} / \mathrm{L}$, respectively) in sample HR-PRFP-04, all VOC results for that sample were coded as "reviewed and rejected" and omitted from this dataset; the coding caused one chloroform detection at a concentration of $0.244 \mu \mathrm{g} / \mathrm{L}$ to be omitted from this report. For HR-SGU-01, all results for organic constituents were coded as "reviewed and rejected" and omitted from this dataset due to suspected chlorination of sample 
Table 6. Pesticides and pesticide degradates detected in samples collected for the Santa Cruz, San Gabriel, and Peninsular Ranges Hard Rock Aquifers (Hard Rock) study unit, March 2011 through March 2012, California GAMA Priority Basin Project.

[The five-digit U.S. Geological Survey (USGS) parameter code below the constituent name is used to uniquely identify a specific constituent or property. Samples from 110 wells were analyzed, but only samples with detections of at least one constituent are listed. Constituents are grouped by primary use or source and listed in order of decreasing detection frequency for each group in the 93 grid wells sampled. All analytes are listed in table 3B. GAMA well identification number: HR-SC, Santa Cruz study area grid well; HR-SG, San Gabriel study area grid well; HR-SGU, San Gabriel study area understanding well; HR-PR, Peninsular Ranges study area grid well; HR-PRFP, Peninsular Ranges study area flow-path well; HR-PRU, Peninsular Ranges study area understanding well. Reporting levels, benchmark types, and benchmark levels as of July 6, 2012. Benchmark type: Maximum contaminant level benchmarks are listed as MCL-US when the MCL-US and MCL-CA are identical, and as MCL-CA when the MCL-CA is lower than the MCL-US or no MCL-US exists. MCL-CA; California Department of Public Health maximum contaminant level. Other abbreviations: E, estimated or having a higher degree of uncertainty; LRL, laboratory reporting level; $\mu \mathrm{g} / \mathrm{L}$, micrograms per liter; na, not available; - , not detected]

\begin{tabular}{|c|c|c|c|c|c|c|c|c|c|c|c|c|c|c|c|c|}
\hline \multirow{2}{*}{$\begin{array}{c}\text { GAMA } \\
\text { well } \\
\text { identification } \\
\text { number }\end{array}$} & \multicolumn{3}{|c|}{ Herbicide degradate } & \multicolumn{7}{|c|}{ Herbicide } & \multicolumn{3}{|c|}{ Insecticide degradates } & \multirow{2}{*}{$\begin{array}{c}\begin{array}{c}\text { Insecti- } \\
\text { cide }\end{array} \\
\begin{array}{c}\text { Carbaryl } \\
(\mu \mathrm{g} / \mathrm{L}) \\
(\mathbf{8 2 6 8 0})\end{array}\end{array}$} & \multirow{2}{*}{$\begin{array}{l}\text { Pes- } \\
\text { ticide } \\
\text { detec- } \\
\text { tions } \\
\text { per well }\end{array}$} & \multirow{2}{*}{$\begin{array}{c}\text { Pes- } \\
\text { ticide } \\
\text { detec- } \\
\text { tion } \\
\text { sum- } \\
\text { mary }\end{array}$} \\
\hline & $\begin{array}{c}\text { Deethyl- } \\
\text { atrazine } \\
(\mu \mathrm{g} / \mathrm{L}) \\
(04040)\end{array}$ & $\begin{array}{c}\text { 3,4-Dichloro- } \\
\text { aniline } \\
(\mu \mathrm{g} / \mathrm{L}) \\
(61625)\end{array}$ & $\begin{array}{c}\text { 2,6-Diethyl- } \\
\text { aniline } \\
(\mu \mathrm{g} / \mathrm{L}) \\
(\mathbf{8 2 6 6 0 )}\end{array}$ & $\begin{array}{c}\text { Sima- } \\
\text { zine } \\
(\mu \mathrm{g} / \mathrm{L}) \\
(04035)\end{array}$ & $\begin{array}{c}\text { Atrazine } \\
(\mu \mathrm{g} / \mathrm{L}) \\
(39632)\end{array}$ & $\begin{array}{l}\text { Tebuthi- } \\
\text { uron } \\
(\mu \mathrm{g} / \mathrm{L}) \\
(82670)\end{array}$ & $\begin{array}{c}\text { Hexazi- } \\
\text { none } \\
(\mu \mathrm{g} / \mathrm{L}) \\
(04025)\end{array}$ & $\begin{array}{l}\text { Metola- } \\
\text { chlor } \\
(\mu \mathrm{g} / \mathrm{L}) \\
(39415)\end{array}$ & $\begin{array}{c}\text { Prome- } \\
\text { ton } \\
(\mu \mathrm{g} / \mathrm{L}) \\
(04037)\end{array}$ & $\begin{array}{c}\text { Me- } \\
\text { tribuzin } \\
(\mu \mathrm{g} / \mathrm{L}) \\
(82630)\end{array}$ & $\begin{array}{c}\text { Desulfinyl- } \\
\text { fipronil } \\
(\mu \mathrm{g} / \mathrm{L}) \\
(\mathbf{6 2 1 7 0})\end{array}$ & $\begin{array}{c}\text { Fipronil } \\
\text { sulfide } \\
(\mu \mathrm{g} / \mathrm{L}) \\
(62167)\end{array}$ & $\begin{array}{c}\text { Fipronil } \\
\text { sulfone } \\
(\mu \mathrm{g} / \mathrm{L}) \\
(62168)\end{array}$ & & & \\
\hline $\begin{array}{l}\text { Benchmark } \\
\text { type }\end{array}$ & na & na & na & MCL-US & MCL-CA & HAL-US & HAL-US & HAL-US & HAL-US & HAL-US & na & na & na & RSD5-US & & \\
\hline $\begin{array}{l}\text { Benchmark } \\
\text { level }\end{array}$ & na & na & na & 4 & 1 & 500 & 400 & 700 & 400 & 70 & na & na & na & 400 & & \\
\hline [LRL] & {$[0.006]$} & {$[0.006]$} & {$[0.0060]$} & {$[0.006]$} & {$[0.008]$} & {$[0.028]$} & {$[0.012]$} & {$[0.020]$} & {$[0.012]$} & {$[0.012]$} & {$[0.012]$} & {$[0.012]$} & {$[0.024]$} & {$[0.06]$} & & \\
\hline \multicolumn{17}{|c|}{ Hard Rock study unit (93 grid wells sampled) ${ }^{1}$} \\
\hline $\begin{array}{l}\text { Number of } \\
\text { wells with } \\
\text { detections }\end{array}$ & 8 & 2 & 1 & 7 & 4 & 3 & 2 & 2 & 2 & 1 & 2 & 1 & 1 & 1 & & 17 \\
\hline $\begin{array}{l}\text { Detection } \\
\text { frequency } \\
\text { (percent) }\end{array}$ & 8.6 & 2.2 & 1.1 & 7.5 & 4.3 & 3.2 & 2.2 & 2.2 & 2.2 & 1.1 & 2.2 & 1.1 & 1.1 & 1.1 & & 18 \\
\hline \multicolumn{17}{|c|}{ Santa Cruz study area ( 25 grid wells sampled) } \\
\hline HR-SC-01 & - & - & - & - & - & - & - & - & - & - & - & - & - & ${ }^{2} \mathrm{E} 0.005$ & 1 & \\
\hline HR-SC-11 & - & - & - & 0.010 & - & - & - & - & - & - & - & - & - & - & 1 & \\
\hline $\begin{array}{l}\text { Number of } \\
\text { wells with } \\
\text { detections }\end{array}$ & 0 & 0 & 0 & 1 & 0 & 0 & 0 & 0 & 0 & 0 & 0 & 0 & 0 & 1 & & 2 \\
\hline $\begin{array}{l}\text { Detection } \\
\text { frequency } \\
\text { (percent) }\end{array}$ & 0 & 0 & 0 & 4.0 & 0 & 0 & 0 & 0 & 0 & 0 & 0 & 0 & 0 & 4.0 & & 8.0 \\
\hline
\end{tabular}


Table 6. Pesticides and pesticide degradates detected in samples collected for the Santa Cruz, San Gabriel, and Peninsular Ranges Hard Rock Aquifers (Hard Rock) study unit, March 2011 through March 2012, California GAMA Priority Basin Project.-Continued

[The five-digit U.S. Geological Survey (USGS) parameter code below the constituent name is used to uniquely identify a specific constituent or property. Samples from 110 wells were analyzed, but only samples with detections of at least one constituent are listed. Constituents are grouped by primary use or source and listed in order of decreasing detection frequency for each group in the 93 grid wells sampled. All analytes are listed in table 3B. GAMA well identification number: HR-SC, Santa Cruz study area grid well; HR-SG, San Gabriel study area grid well; HR-SGU, San Gabriel study area understanding well; HR-PR, Peninsular Ranges study area grid well; HR-PRFP, Peninsular Ranges study area flow-path well; HR-PRU, Peninsular Ranges study area understanding well. Reporting levels, benchmark types, and benchmark levels as of July 6, 2012. Benchmark type: Maximum contaminant level benchmarks are listed as MCL-US when the MCL-US and MCL-CA are identical, and as MCL-CA when the MCL-CA is lower than the MCL-US or no MCL-US exists. MCL-CA; California Department of Public Health maximum contaminant level. Other abbreviations: E, estimated or having a higher degree of uncertainty; LRL, laboratory reporting level; $\mu \mathrm{g} / \mathrm{L}$, micrograms per liter; na, not available; - , not detected]

\begin{tabular}{|c|c|c|c|c|c|c|c|c|c|c|c|c|c|c|c|c|}
\hline \multirow{2}{*}{$\begin{array}{c}\text { GAMA } \\
\text { well } \\
\text { identification } \\
\text { number }\end{array}$} & \multicolumn{3}{|c|}{ Herbicide degradate } & \multicolumn{7}{|c|}{ Herbicide } & \multicolumn{3}{|c|}{ Insecticide degradates } & \multirow{2}{*}{$\begin{array}{c}\text { Insecti- } \\
\text { cide }\end{array}$} & \multirow{2}{*}{$\begin{array}{l}\text { Pes- } \\
\text { ticide } \\
\text { detec- } \\
\text { tions } \\
\text { per well }\end{array}$} & \multirow{2}{*}{$\begin{array}{c}\text { Pes- } \\
\text { ticide } \\
\text { detec- } \\
\text { tion } \\
\text { sum- } \\
\text { mary }\end{array}$} \\
\hline & $\begin{array}{c}\text { Deethyl- } \\
\text { atrazine } \\
(\mu \mathrm{g} / \mathrm{L}) \\
(04040)\end{array}$ & $\begin{array}{c}\text { 3,4-Dichloro- } \\
\text { aniline } \\
\text { ( } \mu \mathrm{g} / \mathrm{L}) \\
(61625)\end{array}$ & $\begin{array}{c}\text { 2,6-Diethyl- } \\
\text { aniline } \\
(\mu \mathrm{g} / \mathrm{L}) \\
(\mathbf{8 2 6 6 0 )}\end{array}$ & $\begin{array}{c}\text { Sima- } \\
\text { zine } \\
(\mu \mathrm{g} / \mathrm{L}) \\
(04035)\end{array}$ & $\begin{array}{c}\text { Atrazine } \\
(\mu \mathrm{g} / \mathrm{L}) \\
(39632)\end{array}$ & $\begin{array}{c}\text { Tebuthi- } \\
\text { uron } \\
(\mu \mathrm{g} / \mathrm{L}) \\
(82670)\end{array}$ & $\begin{array}{c}\text { Hexazi- } \\
\text { none } \\
(\mu \mathrm{g} / \mathrm{L}) \\
(04025)\end{array}$ & $\begin{array}{c}\text { Metola- } \\
\text { chlor } \\
(\mu \mathrm{g} / \mathrm{L}) \\
(39415)\end{array}$ & $\begin{array}{c}\text { Prome- } \\
\text { ton } \\
(\mu \mathrm{g} / \mathrm{L}) \\
(04037)\end{array}$ & $\begin{array}{c}\text { Me- } \\
\text { tribuzin } \\
(\mu \mathrm{g} / \mathrm{L}) \\
(\mathbf{8 2 6 3 0})\end{array}$ & $\begin{array}{c}\text { Desulfinyl- } \\
\text { fipronil } \\
(\mu \mathrm{g} / \mathrm{L}) \\
(62170)\end{array}$ & $\begin{array}{c}\text { Fipronil } \\
\text { sulfide } \\
(\mu \mathrm{g} / \mathrm{L}) \\
(62167)\end{array}$ & $\begin{array}{c}\text { Fipronil } \\
\text { sulfone } \\
(\mu \mathrm{g} / \mathrm{L}) \\
(62168)\end{array}$ & & & \\
\hline $\begin{array}{l}\text { Benchmark } \\
\text { type }\end{array}$ & na & na & na & $\overline{\text { MCL-US }}$ & MCL-CA & HAL-US & HAL-US & HAL-US & HAL-US & HAL-US & na & na & na & RSD5-US & & \\
\hline $\begin{array}{l}\text { Benchmark } \\
\text { level }\end{array}$ & na & na & na & 4 & 1 & 500 & 400 & 700 & 400 & 70 & na & na & na & 400 & & \\
\hline [LRL] & {$[0.006]$} & {$[0.006]$} & {$[0.0060]$} & {$[0.006]$} & {$[0.008]$} & {$[0.028]$} & [0.012] & {$[0.020]$} & {$[0.012]$} & {$[0.012]$} & {$[0.012]$} & {$[0.012]$} & {$[0.024]$} & {$[0.06]$} & & \\
\hline \multicolumn{17}{|c|}{ San Gabriel study area (33 grid wells sampled) } \\
\hline HR-SG-04 & E0.006 & - & - & 0.011 & - & - & - & - & - & - & - & - & - & - & 2 & \\
\hline HR-SG-06 & E0.029 & - & - & - & - & - & - & - & - & - & - & - & - & - & 1 & \\
\hline HR-SG-15 & - & - & - & - & - & - & 0.011 & - & - & - & - & - & - & - & 1 & \\
\hline HR-SG-18 & - & - & - & 0.007 & - & - & - & - & - & - & - & - & - & - & 1 & \\
\hline HR-SG-21 & - & - & - & - & 0.009 & - & - & ${ }^{2} 0.008$ & - & - & - & - & - & - & 2 & \\
\hline $\begin{array}{l}\text { Number of } \\
\text { wells with } \\
\text { detections }\end{array}$ & 2 & 0 & 0 & 2 & 1 & 0 & 1 & 1 & 0 & 0 & 0 & 0 & 0 & 0 & & 5 \\
\hline $\begin{array}{l}\text { Detection } \\
\text { frequency } \\
\text { (percent) }\end{array}$ & 6.1 & 0 & 0 & 6.1 & 3.0 & 0 & 3.0 & 3.0 & 0 & 0 & 0 & 0 & 0 & 0 & & 15 \\
\hline \multicolumn{17}{|c|}{ Peninsular Ranges study area (35 grid wells sampled) } \\
\hline HR-PR-03 & E0.014 & E0.029 & 0.0031 & - & 0.040 & E0.023 & 0.015 & 0.019 & 0.007 & 0.007 & 0.006 & 0.007 & ${ }^{2} \mathrm{E} 0.006$ & - & 12 & \\
\hline HR-PR-06 & - & - & - & - & - & ${ }^{2} 0.009$ & - & - & - & - & - & - & - & - & 1 & \\
\hline HR-PR-07 & E0.005 & - & - & 0.006 & - & - & - & - & - & - & ${ }^{2} 0.003$ & - & - & - & 3 & \\
\hline HR-PR-12 & E0.006 & - & - & 0.008 & 0.005 & - & - & - & - & - & - & - & - & - & 3 & \\
\hline HR-PR-17 & E0.276 & - & - & 0.010 & 0.005 & - & - & - & - & - & - & - & - & - & 3 & \\
\hline
\end{tabular}


Table 6. Pesticides and pesticide degradates detected in samples collected for the Santa Cruz, San Gabriel, and Peninsular Ranges Hard Rock Aquifers (Hard Rock) study unit, March 2011 through March 2012, California GAMA Priority Basin Project.-Continued

[The five-digit U.S. Geological Survey (USGS) parameter code below the constituent name is used to uniquely identify a specific constituent or property. Samples from 110 wells were analyzed, but only samples with detections of at least one constituent are listed. Constituents are grouped by primary use or source and listed in order of decreasing detection frequency for each group in the 93 grid wells sampled. All analytes are listed in table 3B. GAMA well identification number: HR-SC, Santa Cruz study area grid well; HR-SG, San Gabriel study area grid well; HR-SGU, San Gabriel study area understanding well; HR-PR, Peninsular Ranges study area grid well; HR-PRFP, Peninsular Ranges study area flow-path well; HR-PRU, Peninsular Ranges study area understanding well. Reporting levels, benchmark types, and benchmark levels as of July 6, 2012. Benchmark type: Maximum contaminant level benchmarks are listed as MCL-US when the MCL-US and MCL-CA are identical, and as MCL-CA when the MCL-CA is lower than the MCL-US or no MCL-US exists. MCL-CA; California Department of Public Health maximum contaminant level. Other abbreviations: E, estimated or having a higher degree of uncertainty; LRL, laboratory reporting level; $\mu \mathrm{g} / \mathrm{L}$, micrograms per liter; na, not available; - , not detected]

\begin{tabular}{|c|c|c|c|c|c|c|c|c|c|c|c|c|c|c|c|c|}
\hline \multirow{2}{*}{$\begin{array}{c}\text { GAMA } \\
\text { well } \\
\text { identification } \\
\text { number }\end{array}$} & \multicolumn{3}{|c|}{ Herbicide degradate } & \multicolumn{7}{|c|}{ Herbicide } & \multicolumn{3}{|c|}{ Insecticide degradates } & \multirow{2}{*}{$\begin{array}{c}\begin{array}{c}\text { Insecti- } \\
\text { cide }\end{array} \\
\text { Carbaryl } \\
(\mu \mathrm{g} / \mathrm{L}) \\
(82680)\end{array}$} & \multirow{2}{*}{$\begin{array}{c}\text { Pes- } \\
\text { ticide } \\
\text { detec- } \\
\text { tions } \\
\text { per well }\end{array}$} & \multirow{2}{*}{$\begin{array}{c}\text { Pes- } \\
\text { ticide } \\
\text { detec- } \\
\text { tion } \\
\text { sum- } \\
\text { mary }\end{array}$} \\
\hline & $\begin{array}{c}\text { Deethyl- } \\
\text { atrazine } \\
(\mu \mathrm{g} / \mathrm{L}) \\
(04040)\end{array}$ & $\begin{array}{c}\text { 3,4-Dichloro- } \\
\text { aniline } \\
(\mu \mathrm{g} / \mathrm{L}) \\
(61625)\end{array}$ & $\begin{array}{c}\text { 2,6-Diethyl- } \\
\text { aniline } \\
(\mu \mathrm{g} / \mathrm{L}) \\
(82660)\end{array}$ & $\begin{array}{c}\text { Sima- } \\
\text { zine } \\
(\mu \mathrm{g} / \mathrm{L}) \\
(04035)\end{array}$ & $\begin{array}{c}\text { Atrazine } \\
\text { ( } \mu \mathrm{g} / \mathrm{L}) \\
(39632)\end{array}$ & $\begin{array}{c}\text { Tebuthi- } \\
\text { uron } \\
(\mu \mathrm{g} / \mathrm{L}) \\
(82670)\end{array}$ & $\begin{array}{c}\text { Hexazi- } \\
\text { none } \\
(\mu \mathrm{g} / \mathrm{L}) \\
(04025)\end{array}$ & $\begin{array}{c}\text { Metola- } \\
\text { chlor } \\
(\mu \mathrm{g} / \mathrm{L}) \\
(39415)\end{array}$ & $\begin{array}{c}\text { Prome- } \\
\text { ton } \\
(\mu \mathrm{g} / \mathrm{L}) \\
(04037)\end{array}$ & $\begin{array}{c}\text { Me- } \\
\text { tribuzin } \\
(\mu \mathrm{g} / \mathrm{L}) \\
(82630)\end{array}$ & $\begin{array}{c}\text { Desulfinyl- } \\
\text { fipronil } \\
(\mu \mathrm{g} / \mathrm{L}) \\
(62170)\end{array}$ & $\begin{array}{c}\text { Fipronil } \\
\text { sulfide } \\
(\mu \mathrm{g} / \mathrm{L}) \\
(62167)\end{array}$ & $\begin{array}{c}\text { Fipronil } \\
\text { sulfone } \\
(\mu \mathrm{g} / \mathrm{L}) \\
(62168)\end{array}$ & & & \\
\hline $\begin{array}{l}\text { Benchmark } \\
\text { type }\end{array}$ & na & na & na & MCL-US & MCL-CA & HAL-US & HAL-US & HAL-US & HAL-US & HAL-US & na & na & na & RSD5-US & & \\
\hline $\begin{array}{l}\text { Benchmark } \\
\text { level }\end{array}$ & na & na & na & 4 & 1 & 500 & 400 & 700 & 400 & 70 & na & na & na & 400 & & \\
\hline [LRL] & {$[0.006]$} & {$[0.006]$} & {$[0.0060]$} & {$[0.006]$} & {$[0.008]$} & {$[0.028]$} & {$[0.012]$} & {$[0.020]$} & {$[0.012]$} & {$[0.012]$} & {$[0.012]$} & {$[0.012]$} & {$[0.024]$} & {$[0.06]$} & & \\
\hline \multicolumn{17}{|c|}{ Peninsular Ranges study area (35 grid wells sampled) —Continued } \\
\hline HR-PR-21 & E0.006 & - & - & - & - & E0.031 & - & - & - & - & - & - & - & - & 2 & \\
\hline HR-PR-24 & E0.005 & - & - & - & - & - & - & - & - & - & - & - & - & - & 1 & \\
\hline HR-PR-26 & - & - & - & 0.006 & - & - & - & - & - & - & - & - & - & - & 1 & \\
\hline HR-PR-28 & - & E0.0036 & - & - & - & - & - & - & - & - & - & - & - & - & 1 & \\
\hline HR-PR-33 & - & - & - & - & - & - & - & - & ${ }^{2} 0.005$ & - & - & - & - & - & 1 & \\
\hline $\begin{array}{l}\text { Number of } \\
\text { wells with } \\
\text { detections }\end{array}$ & 6 & 2 & 1 & 4 & 3 & 3 & 1 & 1 & 2 & 1 & 2 & 1 & 1 & 0 & & 10 \\
\hline $\begin{array}{l}\text { Detection } \\
\text { frequency } \\
\text { (percent) }\end{array}$ & 17 & 5.7 & 2.9 & 11 & 8.6 & 8.6 & 2.9 & 2.9 & 5.7 & 2.9 & 5.7 & 2.9 & 2.9 & 0 & & 29 \\
\hline \multicolumn{17}{|c|}{ Hard Rock understanding wells (17 wells sampled) ${ }^{3}$} \\
\hline HR-PRFP-05 & - & - & - & 0.006 & - & - & - & - & 0.007 & - & - & - & - & - & 2 & \\
\hline HR-PRFP-11 & - & - & - & - & - & - & - & - & 0.006 & - & - & - & - & - & 1 & \\
\hline HR-PRFP-12 & E0.035 & - & - & 0.015 & 0.009 & - & - & - & 0.007 & - & - & - & - & - & 4 & \\
\hline HR-PRFP-13 & - & - & - & - & - & E0.015 & - & - & - & - & - & - & - & - & 1 & \\
\hline
\end{tabular}

${ }^{1}$ One grid-well pesticide sample (HR-PR-01) was ruined during analysis.

${ }^{2}$ Result is below long-term method detection level.

${ }^{3}$ For sample HR-SGU-01, all results for organic constituents were coded as "reviewed and rejected" and omitted from this dataset due to suspected chlorination of sample. 
Table 7. Perchlorate detected in samples collected for the Santa Cruz, San Gabriel, and Peninsular Ranges Hard Rock Aquifers (Hard Rock) study unit, March 2011 through March 2012, California GAMA Priority Basin Project.

[The five-digit U.S. Geological Survey (USGS) parameter code below the constituent name is used to uniquely identify a specific constituent or property. Information about the constituent is given in table 3C. Samples from all 112 wells were analyzed, but only samples with detections are listed. GAMA well identification number: HR-SC, Santa Cruz study area grid well; HR-SG, San Gabriel study area grid well; HR-SGU, San Gabriel study area understanding well; HR-PR, Peninsular Ranges study area grid well; HR-PRFP, Peninsular Ranges study area flow-path well; HR-PRU, Peninsular Ranges study area understanding well. Reporting level, benchmark type, and benchmark level as of July 6, 2012. Benchmark type: Maximum contaminant level benchmarks are listed as MCL-US when the MCL-US and MCL-CA are identical, and as MCL-CA when the MCL-CA is lower than the MCL-US or no MCL-US exists. MCL-CA, California Department of Public Health maximum contaminant level. Other abbreviations: MRL, minimum reporting level; $\mu \mathrm{g} / \mathrm{L}, \mathrm{micrograms}$ per liter]

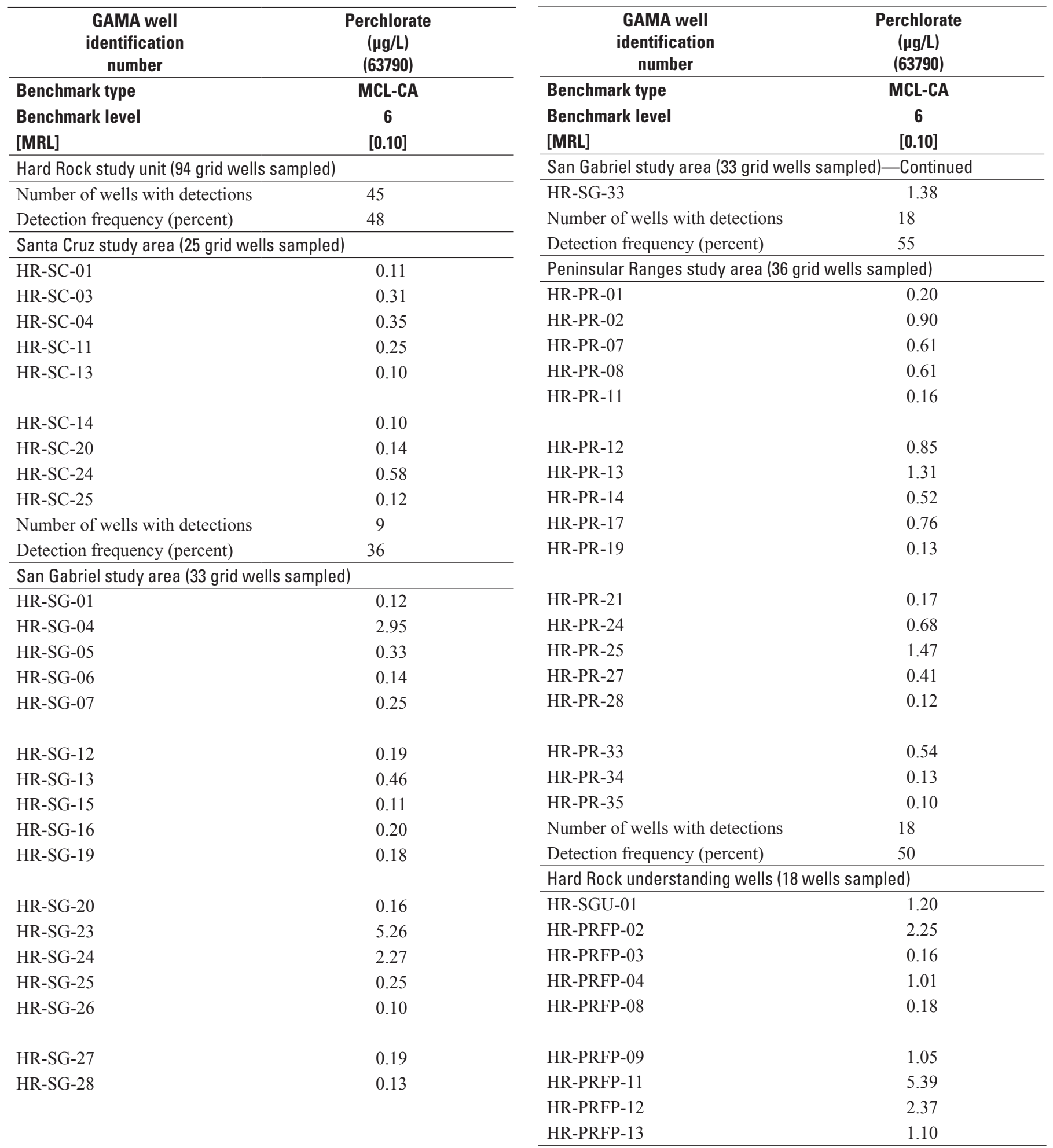


Table 8. Trace elements detected in the samples collected for the Santa Cruz, San Gabriel, and Peninsular Ranges Hard Rock Aquifers (Hard Rock) study unit, March 2011 through March 2012, California GAMA Priority Basin Project.

[The five-digit U.S. Geological Survey (USGS) parameter code below the constituent name is used to uniquely identify a specific constituent or property. GAMA well identification number: HR-SC, Santa Cruz study area grid well; HR-SG, San Gabriel study area grid well; HR-SGU, San Gabriel study area understanding well; HR-PR, Peninsular Ranges study area grid well; HR-PRFP, Peninsular Ranges study area flow-path well; HR-PRU, Peninsular Ranges study area understanding well. Reporting levels, benchmark types, and benchmark levels as of July 6, 2012. Benchmark type: Maximum contaminant level benchmarks are listed as MCL-US when the MCL-US and MCL-CA are identical, and as MCL-CA when the MCL-CA is lower than the MCL-US or no MCL-US exists. Secondary maximum contaminant level benchmarks are listed as SMCL-CA when the SMCL-CA exists, and as MCL-US when the SMCL-CA does not exist. AL-US, U.S. Environmental Protection Agency (USEPA) action level; HAL-US, USEPA lifetime health advisory level; MCL-CA, California Department of Public Health (CDPH) maximum contaminant level; MCL-US, USEPA maximum contaminant level; NL-CA, CDPH notification level;

SMCL-CA, CDPH secondary maximum contaminant level. Other abbreviations: LT-MDL, long-term method detection level; SRL, study reporting level; $\mu \mathrm{g} / \mathrm{L}$, micrograms per liter; - , not detected; $\leq$, less than or equal to; *, value above benchmark level]

\begin{tabular}{|c|c|c|c|c|c|c|c|c|c|c|c|c|}
\hline $\begin{array}{c}\text { GAMA well } \\
\text { identification } \\
\text { number }\end{array}$ & $\begin{array}{c}\text { Aluminum } \\
(\mu \mathrm{g} / \mathrm{L}) \\
(01106)\end{array}$ & $\begin{array}{c}\text { Antimony } \\
(\mu \mathrm{g} / \mathrm{L}) \\
(01095)\end{array}$ & $\begin{array}{c}\text { Arsenic } \\
(\mu \mathrm{g} / \mathrm{L}) \\
(01000)\end{array}$ & $\begin{array}{c}\text { Barium } \\
(\mu \mathrm{g} / \mathrm{L}) \\
(01005)\end{array}$ & $\begin{array}{c}\text { Beryllium } \\
(\mu \mathrm{g} / \mathrm{L}) \\
(01010)\end{array}$ & $\begin{array}{l}\text { Boron } \\
\text { ( } \mu \mathrm{g} / \mathrm{L}) \\
(01020)\end{array}$ & $\begin{array}{c}\text { Cadmium } \\
\text { ( } \mu \mathrm{g} / \mathrm{L}) \\
(01025)\end{array}$ & $\begin{array}{c}\text { Chromium } \\
(\mu \mathrm{g} / \mathrm{L}) \\
(01030)\end{array}$ & $\begin{array}{l}\text { Copper } \\
\text { ( } \mu \mathrm{g} / \mathrm{L}) \\
(01040)\end{array}$ & $\begin{array}{c}\text { Iron } \\
(\mu \mathrm{g} / \mathrm{L}) \\
(01046)\end{array}$ & $\begin{array}{c}\text { Lead } \\
(\mu \mathrm{g} / \mathrm{L}) \\
(01049)\end{array}$ & $\begin{array}{c}\text { Lithium } \\
(\mu \mathrm{g} / \mathrm{L}) \\
(01130)\end{array}$ \\
\hline Benchmark type & MCL-CA & MCL-US & MCL-US & MCL-CA & MCL-US & NL-CA & MCL-US & MCL-CA & AL-US & SMCL-CA & AL-US & na \\
\hline Benchmark level & 1,000 & 6 & 10 & 1,000 & 4 & 1,000 & 5 & 50 & 1,300 & 300 & 15 & na \\
\hline [LT-MDL] or [SRL] & [2.2] & {$[0.027]$} & {$[0.03]$} & {$[0.07]$} & {$[0.006]$} & [3] & {$[0.016]$} & {$[0.07]$} & ${ }^{1}[2.1]$ & ${ }^{1}[6]$ & ${ }^{1}[0.82]$ & {$[0.22]$} \\
\hline \multicolumn{13}{|c|}{ Santa Cruz study area ( 25 wells sampled) } \\
\hline HR-SC-01 & 2.2 & - & 7.9 & 26.2 & - & 13 & 0.045 & - & $\leq 1.0$ & - & $\leq 0.120$ & 0.76 \\
\hline HR-SC-02 & 291 & 0.034 & 0.23 & 44.0 & 0.019 & 83 & 0.023 & 0.30 & 3.4 & 266 & $\leq 0.373$ & 16.9 \\
\hline HR-SC-03 & - & - & $* 20.0$ & 95.7 & 0.026 & $* 1,980$ & 0.205 & 0.07 & $\leq 0.52$ & $* 8,390$ & $\leq 0.092$ & 4.86 \\
\hline HR-SC-04 & - & 0.358 & 0.13 & 38.8 & 0.006 & 142 & - & 0.07 & $\leq 1.2$ & $\leq 4.9$ & $\leq 0.128$ & 24.6 \\
\hline HR-SC-05 & - & - & 0.05 & 15.2 & 0.018 & 10 & - & - & - & $* 1,010$ & - & 16.0 \\
\hline HR-SC-06 & ${ }^{2} 1.7$ & - & 0.08 & 13.9 & 0.006 & 54 & - & - & 2.4 & 5.9 & $\leq 0.137$ & 21.8 \\
\hline HR-SC-07 & 3.0 & 0.067 & 0.20 & 23.6 & 0.011 & 425 & - & 0.10 & - & 276 & $\leq 0.024$ & 103 \\
\hline HR-SC-08 & 3.4 & 0.046 & 0.11 & 190 & 0.019 & 52 & 0.035 & - & 5.5 & * 447 & $\leq 0.322$ & 19.0 \\
\hline HR-SC-09 & 54.0 & - & 1.0 & 116 & 0.217 & $* 7,920$ & - & - & - & 42.8 & $\leq 0.190$ & 187 \\
\hline HR-SC-10 & ${ }^{2} 2.0$ & 0.037 & 0.24 & 0.66 & - & 9 & - & - & - & 7.0 & $\leq 0.642$ & 8.13 \\
\hline HR-SC-11 & - & 0.039 & 0.12 & 281 & - & 43 & - & 0.09 & 4.1 & - & 4.86 & 2.56 \\
\hline HR-SC-12 & - & - & 0.05 & 194 & 0.010 & 897 & - & - & $\leq 0.50$ & 166 & $\leq 0.747$ & 5.43 \\
\hline HR-SC-13 & 4.3 & 0.136 & 0.30 & 1.48 & - & 151 & - & 0.10 & $\leq 1.9$ & 13.1 & $\leq 0.305$ & 3.51 \\
\hline HR-SC-14 & 2.3 & 0.034 & 0.16 & 39.1 & - & 31 & 0.019 & 0.08 & - & * 940 & $\leq 0.399$ & 24.6 \\
\hline HR-SC-15 & 6.4 & 3.63 & * 19.1 & 2.35 & - & 101 & - & - & $\leq 1.1$ & - & $\leq 0.718$ & 36.4 \\
\hline
\end{tabular}


Table 8. Trace elements detected in the samples collected for the Santa Cruz, San Gabriel, and Peninsular Ranges Hard Rock Aquifers (Hard Rock) study unit, March 2011 through March 2012, California GAMA Priority Basin Project.-Continued

[The five-digit U.S. Geological Survey (USGS) parameter code below the constituent name is used to uniquely identify a specific constituent or property. GAMA well identification number: HR-SC, Santa Cruz study area grid well; HR-SG, San Gabriel study area grid well; HR-SGU, San Gabriel study area understanding well; HR-PR, Peninsular Ranges study area grid well; HR-PRFP, Peninsular Ranges study area flow-path well; HR-PRU, Peninsular Ranges study area understanding well. Reporting levels, benchmark types, and benchmark levels as of July 6, 2012. Benchmark type: Maximum contaminant level benchmarks are listed as MCL-US when the MCL-US and MCL-CA are identical, and as MCL-CA when the MCL-CA is lower than the MCL-US or no MCL-US exists. Secondary maximum contaminant leve benchmarks are listed as SMCL-CA when the SMCL-CA exists, and as MCL-US when the SMCL-CA does not exist. AL-US, U.S. Environmental Protection Agency (USEPA) action level; HAL-US, USEPA lifetime health advisory level; MCL-CA, California Department of Public Health (CDPH) maximum contaminant level; MCL-US, USEPA maximum contaminant level; NL-CA, CDPH notification level; SMCL-CA, CDPH secondary maximum contaminant level. Other abbreviations: LT-MDL, long-term method detection level; SRL, study reporting level; $\mu \mathrm{g} / \mathrm{L}$, micrograms per liter; - , not detected; $\leq$, less than or equal to; *, value above benchmark level]

\begin{tabular}{|c|c|c|c|c|c|c|c|c|c|c|c|c|}
\hline $\begin{array}{c}\text { GAMA well } \\
\text { identification } \\
\text { number }\end{array}$ & $\begin{array}{c}\text { Aluminum } \\
(\mu \mathrm{g} / \mathrm{L}) \\
(01106)\end{array}$ & $\begin{array}{c}\text { Antimony } \\
(\mu \mathrm{g} / \mathrm{L}) \\
(01095)\end{array}$ & $\begin{array}{c}\text { Arsenic } \\
(\mu \mathrm{g} / \mathrm{L}) \\
(01000)\end{array}$ & $\begin{array}{l}\text { Barium } \\
\text { ( } \mu \mathrm{g} / \mathrm{L}) \\
(01005)\end{array}$ & $\begin{array}{c}\text { Beryllium } \\
(\mu \mathrm{g} / \mathrm{L}) \\
(01010)\end{array}$ & $\begin{array}{l}\text { Boron } \\
(\mu \mathrm{g} / \mathrm{L}) \\
(01020)\end{array}$ & $\begin{array}{c}\text { Cadmium } \\
(\mu \mathrm{g} / \mathrm{L}) \\
(01025)\end{array}$ & $\begin{array}{c}\text { Chromium } \\
(\mu \mathrm{g} / \mathrm{L}) \\
(01030)\end{array}$ & $\begin{array}{l}\text { Copper } \\
\text { ( } \mu \mathrm{g} / \mathrm{L}) \\
(01040)\end{array}$ & $\begin{array}{c}\text { Iron } \\
(\mu \mathrm{g} / \mathrm{L}) \\
(01046)\end{array}$ & $\begin{array}{c}\text { Lead } \\
\text { ( } \mu \mathrm{g} / \mathrm{L}) \\
(01049)\end{array}$ & $\begin{array}{c}\text { Lithium } \\
(\mu \mathrm{g} / \mathrm{L}) \\
(01130)\end{array}$ \\
\hline Benchmark type & MCL-CA & MCL-US & MCL-US & MCL-CA & MCL-US & NL-CA & MCL-US & MCL-CA & AL-US & SMCL-CA & AL-US & na \\
\hline Benchmark level & 1,000 & 6 & 10 & 1,000 & 4 & 1,000 & 5 & 50 & 1,300 & 300 & 15 & na \\
\hline [LT-MDL] or [SRL] & [2.2] & {$[0.027]$} & {$[0.03]$} & {$[0.07]$} & {$[0.006]$} & [3] & {$[0.016]$} & {$[0.07]$} & ${ }^{1}[2.1]$ & ${ }^{1}[6]$ & ${ }^{1}[0.82]$ & {$[0.22]$} \\
\hline \multicolumn{13}{|c|}{ Santa Cruz study area (25 wells sampled) —Continued } \\
\hline HR-SC-16 & - & 0.550 & 1.2 & 10.7 & - & 14 & - & - & $\leq 0.77$ & - & $\leq 0.332$ & 15.3 \\
\hline HR-SC-17 & 2.3 & - & 0.27 & 10.9 & 0.032 & 7 & 0.537 & 0.24 & 4.9 & 37.8 & $\leq 0.101$ & 2.03 \\
\hline HR-SC-18 & - & - & 8.9 & 18.3 & - & 15 & - & 0.11 & $\leq 0.67$ & * 341 & - & 4.68 \\
\hline HR-SC-19 & 2.2 & - & 0.25 & 59.4 & 0.018 & 491 & - & - & - & $* 1,250$ & $\leq 0.023$ & 45.3 \\
\hline HR-SC-20 & - & 0.055 & 0.47 & 12.2 & - & 221 & 0.029 & 1.4 & 4.0 & 8.2 & $\leq 0.632$ & 56.1 \\
\hline HR-SC-21 & - & - & 1.1 & 9.94 & 0.015 & 786 & - & - & - & $* 2,330$ & $\leq 0.023$ & 44.3 \\
\hline HR-SC-22 & - & 0.030 & 0.07 & 110 & 0.017 & 53 & - & - & - & $* 1,490$ & 1.54 & 43.0 \\
\hline HR-SC-23 & - & - & 0.63 & 73.9 & 0.008 & 329 & - & - & - & 99.0 & $\leq 0.185$ & 98.0 \\
\hline HR-SC-24 & - & 0.061 & 0.62 & 132 & 0.011 & 418 & 0.019 & 2.4 & $\leq 1.9$ & 83.1 & 4.92 & 54.7 \\
\hline HR-SC-25 & 8.9 & 0.112 & 1.8 & 1.17 & - & 15 & 0.185 & 0.28 & 5.4 & - & 1.01 & 2.98 \\
\hline \multicolumn{13}{|c|}{ San Gabriel study area (37 wells sampled) } \\
\hline HR-SG-01 & - & 0.065 & 0.51 & 58.0 & - & 101 & 0.021 & 0.07 & $\leq 1.8$ & $\leq 5.2$ & $\leq 0.393$ & 4.42 \\
\hline HR-SG-02 & - & - & 0.12 & 48.1 & - & 46 & 0.023 & 0.07 & - & 84.8 & $\leq 0.117$ & 1.13 \\
\hline HR-SG-03 & 4.3 & 0.050 & 0.58 & 75.5 & - & 144 & - & 0.08 & $\leq 1.9$ & $\leq 3.6$ & $\leq 0.306$ & 0.24 \\
\hline HR-SG-04 & - & - & 0.35 & 124 & - & 138 & - & 0.64 & - & $\leq 5.6$ & $\leq 0.556$ & 10.4 \\
\hline HR-SG-05 ${ }^{3}$ & - & - & - & 44.5 & - & 44 & - & 0.56 & - & 6.4 & $\leq 0.077$ & 4.41 \\
\hline HR-SG-06 & 2.2 & 0.502 & 4.5 & 7.52 & - & 42 & - & 0.08 & - & - & - & 5.97 \\
\hline HR-SG-07 & 2.5 & - & 0.64 & 4.06 & 0.029 & 23 & - & - & - & $* 484$ & $\leq 0.016$ & 13.7 \\
\hline HR-SG-08 & - & 0.029 & 0.87 & 99.6 & - & 210 & - & - & $\leq 1.1$ & 35.4 & $\leq 0.068$ & 0.23 \\
\hline HR-SG-09 & ${ }^{2} 1.8$ & 0.086 & $* 14.7$ & 4.49 & - & 411 & - & 0.11 & $\leq 0.84$ & 16.6 & 1.20 & 30.1 \\
\hline HR-SG-10 & - & - & 0.07 & 21.8 & - & 44 & 0.023 & 0.46 & $\leq 1.1$ & $\leq 4.8$ & $\leq 0.428$ & 13.1 \\
\hline
\end{tabular}


Table 8. Trace elements detected in the samples collected for the Santa Cruz, San Gabriel, and Peninsular Ranges Hard Rock Aquifers (Hard Rock) study unit, March 2011 through March 2012, California GAMA Priority Basin Project.-Continued

[The five-digit U.S. Geological Survey (USGS) parameter code below the constituent name is used to uniquely identify a specific constituent or property. GAMA well identification number: HR-SC, Santa Cruz study area grid well; HR-SG, San Gabriel study area grid well; HR-SGU, San Gabriel study area understanding well; HR-PR, Peninsular Ranges study area grid well; HR-PRFP, Peninsular Ranges study area flow-path well; HR-PRU, Peninsular Ranges study area understanding well. Reporting levels, benchmark types, and benchmark levels as of July 6, 2012. Benchmark type: Maximum contaminant level benchmarks are listed as MCL-US when the MCL-US and MCL-CA are identical, and as MCL-CA when the MCL-CA is lower than the MCL-US or no MCL-US exists. Secondary maximum contaminant level benchmarks are listed as SMCL-CA when the SMCL-CA exists, and as MCL-US when the SMCL-CA does not exist. AL-US, U.S. Environmental Protection Agency (USEPA) action level; HAL-US, USEPA lifetime health advisory level; MCL-CA, California Department of Public Health (CDPH) maximum contaminant level; MCL-US, USEPA maximum contaminant level; NL-CA, CDPH notification level;

SMCL-CA, CDPH secondary maximum contaminant level. Other abbreviations: LT-MDL, long-term method detection level; SRL, study reporting level; $\mu \mathrm{g} / \mathrm{L}$, micrograms per liter; —, not detected; $\leq$, less than or equal to; *, value above benchmark level]

\begin{tabular}{|c|c|c|c|c|c|c|c|c|c|c|c|c|}
\hline $\begin{array}{c}\text { GAMA well } \\
\text { identification } \\
\text { number }\end{array}$ & $\begin{array}{c}\text { Aluminum } \\
(\mu \mathrm{g} / \mathrm{L}) \\
(01106)\end{array}$ & $\begin{array}{c}\text { Antimony } \\
(\mu \mathrm{g} / \mathrm{L}) \\
(01095)\end{array}$ & $\begin{array}{c}\text { Arsenic } \\
(\mu \mathrm{g} / \mathrm{L}) \\
(01000)\end{array}$ & $\begin{array}{c}\text { Barium } \\
(\mu \mathrm{g} / \mathrm{L}) \\
(01005)\end{array}$ & $\begin{array}{c}\text { Beryllium } \\
(\mu \mathrm{g} / \mathrm{L}) \\
(01010)\end{array}$ & $\begin{array}{c}\text { Boron } \\
(\mu \mathrm{g} / \mathrm{L}) \\
(01020)\end{array}$ & $\begin{array}{c}\text { Cadmium } \\
(\mu \mathrm{g} / \mathrm{L}) \\
(01025)\end{array}$ & $\begin{array}{c}\text { Chromium } \\
(\mu \mathrm{g} / \mathrm{L}) \\
(01030)\end{array}$ & $\begin{array}{c}\text { Copper } \\
(\mu \mathrm{g} / \mathrm{L}) \\
(01040)\end{array}$ & $\begin{array}{c}\text { Iron } \\
\text { (pg/L) } \\
(01046)\end{array}$ & $\begin{array}{c}\text { Lead } \\
(\mu \mathrm{g} / \mathrm{L}) \\
(01049)\end{array}$ & $\begin{array}{c}\text { Lithium } \\
(\mu \mathrm{g} / \mathrm{L}) \\
(01130)\end{array}$ \\
\hline Benchmark type & MCL-CA & MCL-US & MCL-US & MCL-CA & MCL-US & NL-CA & MCL-US & MCL-CA & AL-US & SMCL-CA & AL-US & na \\
\hline Benchmark level & 1,000 & 6 & 10 & 1,000 & 4 & 1,000 & 5 & 50 & 1,300 & 300 & 15 & na \\
\hline [LT-MDL] or [SRL] & [2.2] & {$[0.027]$} & {$[0.03]$} & {$[0.07]$} & {$[0.006]$} & [3] & {$[0.016]$} & {$[0.07]$} & ${ }^{1}[2.1]$ & ${ }^{1}[6]$ & ${ }^{1}[0.82]$ & [0.22] \\
\hline \multicolumn{13}{|c|}{ San Gabriel study area (37 wells sampled)_Continued } \\
\hline HR-SG-11 & 23.7 & - & 0.09 & 29.0 & - & 39 & - & 0.12 & - & 9.8 & $\leq 0.065$ & 17.4 \\
\hline HR-SG-12 & - & 0.238 & 0.55 & 25.0 & - & 23 & - & 0.16 & $\leq 1.1$ & - & 0.941 & 4.09 \\
\hline HR-SG-13 & - & - & 0.13 & 33.0 & - & 37 & - & 0.21 & $\leq 1.2$ & $\leq 5.2$ & $\leq 0.145$ & 6.62 \\
\hline HR-SG-14 & - & - & 0.03 & 5.91 & 0.017 & 13 & - & - & $\leq 2.0$ & $* 457$ & $\leq 0.264$ & 10.3 \\
\hline HR-SG-15 & - & 1.25 & 0.11 & 44.7 & - & 50 & 0.082 & 0.07 & $\leq 1.8$ & - & $\leq 0.294$ & 1.98 \\
\hline HR-SG-16 & ${ }^{2} 2.0$ & 3.85 & 2.6 & 77.7 & - & 92 & 0.020 & 0.08 & 3.8 & - & $\leq 0.644$ & 21.9 \\
\hline HR-SG-17 & - & 0.033 & 0.06 & 2.63 & 0.042 & 5 & - & - & 2.6 & - & 0.827 & 54.1 \\
\hline HR-SG-18 & - & 0.070 & 0.58 & 24.1 & - & 17 & - & - & 4.2 & $\leq 5.2$ & $\leq 0.078$ & 0.39 \\
\hline HR-SG-19 & 2.2 & - & - & 77.4 & - & 16 & - & - & $\leq 1.7$ & 10.3 & $\leq 0.224$ & 11.9 \\
\hline HR-SG-20 & - & 0.154 & 0.07 & 82.2 & - & 110 & 0.044 & 0.11 & 2.4 & 8.0 & 1.83 & 14.3 \\
\hline HR-SG-21 & - & - & 0.07 & 0.92 & - & 4 & 0.021 & - & $\leq 1.2$ & 73.9 & $\leq 0.373$ & - \\
\hline HR-SG-22 & - & 0.278 & 1.3 & 72.2 & - & 418 & 0.031 & - & $\leq 1.7$ & $\leq 4.2$ & 0.868 & 50.8 \\
\hline HR-SG-23 & ${ }^{2} 1.8$ & 0.041 & 0.26 & 10.7 & - & 11 & 0.018 & 0.10 & - & - & $\leq 0.068$ & 4.25 \\
\hline HR-SG-24 & ${ }^{2} 2.0$ & 0.117 & 2.4 & 18.9 & 0.022 & $* 1,530$ & 0.147 & 0.07 & - & $\leq 3.8$ & $\leq 0.125$ & 10.3 \\
\hline HR-SG-25 & 5.0 & - & 0.32 & 23.9 & - & 4 & - & 0.10 & - & $\leq 5.6$ & $\leq 0.019$ & 58.2 \\
\hline HR-SG-26 & 3.7 & 0.404 & 3.3 & 89.1 & - & 28 & - & 0.16 & - & - & $\leq 0.045$ & 1.81 \\
\hline HR-SG-27 & - & 0.097 & 0.28 & 8.82 & - & 4 & - & 0.07 & 5.3 & 15.4 & $\leq 0.709$ & 21.8 \\
\hline HR-SG-28 & - & - & - & 7.62 & - & 9 & - & 0.12 & 2.2 & - & $\leq 0.296$ & 0.85 \\
\hline HR-SG-29 & - & 0.222 & 2.2 & 60.3 & 0.011 & - & 0.036 & - & $\leq 1.3$ & 72.5 & $\leq 0.421$ & 2.41 \\
\hline HR-SG-30 & - & 0.106 & 0.93 & 63.8 & - & 3 & - & 0.19 & $\leq 1.3$ & - & 2.56 & 1.89 \\
\hline
\end{tabular}


Table 8. Trace elements detected in the samples collected for the Santa Cruz, San Gabriel, and Peninsular Ranges Hard Rock Aquifers (Hard Rock) study unit, March 2011 through March 2012, California GAMA Priority Basin Project.-Continued

[The five-digit U.S. Geological Survey (USGS) parameter code below the constituent name is used to uniquely identify a specific constituent or property. GAMA well identification number: HR-SC, Santa Cruz study area grid well; HR-SG, San Gabriel study area grid well; HR-SGU, San Gabriel study area understanding well; HR-PR, Peninsular Ranges study area grid well; HR-PRFP, Peninsular Ranges study area flow-path well; HR-PRU, Peninsular Ranges study area understanding well. Reporting levels, benchmark types, and benchmark levels as of July 6, 2012. Benchmark type: Maximum contaminant level benchmarks are listed as MCL-US when the MCL-US and MCL-CA are identical, and as MCL-CA when the MCL-CA is lower than the MCL-US or no MCL-US exists. Secondary maximum contaminant level benchmarks are listed as SMCL-CA when the SMCL-CA exists, and as MCL-US when the SMCL-CA does not exist. AL-US, U.S. Environmental Protection Agency (USEPA) action level; HAL-US, USEPA lifetime health advisory level; MCL-CA, California Department of Public Health (CDPH) maximum contaminant level; MCL-US, USEPA maximum contaminant level; NL-CA, CDPH notification level; SMCL-CA, CDPH secondary maximum contaminant level. Other abbreviations: LT-MDL, long-term method detection level; SRL, study reporting level; $\mu \mathrm{g} / \mathrm{L}$, micrograms per liter; - , not detected; $\leq$ less than or equal to; *, value above benchmark level]

\begin{tabular}{|c|c|c|c|c|c|c|c|c|c|c|c|c|}
\hline $\begin{array}{c}\text { GAMA well } \\
\text { identification } \\
\text { number }\end{array}$ & $\begin{array}{c}\text { Aluminum } \\
(\mu \mathrm{g} / \mathrm{L}) \\
(01106)\end{array}$ & $\begin{array}{c}\text { Antimony } \\
(\mu \mathrm{g} / \mathrm{L}) \\
(01095)\end{array}$ & $\begin{array}{c}\text { Arsenic } \\
(\mu \mathrm{g} / \mathrm{L}) \\
(01000)\end{array}$ & $\begin{array}{c}\text { Barium } \\
(\mu \mathrm{g} / \mathrm{L}) \\
(01005)\end{array}$ & $\begin{array}{c}\text { Beryllium } \\
\text { ( } \mu \mathrm{g} / \mathrm{L}) \\
(01010)\end{array}$ & $\begin{array}{c}\text { Boron } \\
(\mu g / L) \\
(01020)\end{array}$ & $\begin{array}{c}\text { Cadmium } \\
(\mu \mathrm{g} / \mathrm{L}) \\
(01025)\end{array}$ & $\begin{array}{c}\text { Chromium } \\
(\mu \mathrm{g} / \mathrm{L}) \\
(01030)\end{array}$ & $\begin{array}{c}\text { Copper } \\
\text { ( } \mu \mathrm{g} / \mathrm{L}) \\
(01040)\end{array}$ & $\begin{array}{c}\text { Iron } \\
(\mu \mathrm{gg} / \mathrm{L}) \\
(01046)\end{array}$ & $\begin{array}{c}\text { Lead } \\
(\mu \mathrm{g} / \mathrm{L}) \\
(01049)\end{array}$ & $\begin{array}{c}\text { Lithium } \\
(\mu \mathrm{g} / \mathrm{L}) \\
(01130)\end{array}$ \\
\hline Benchmark type & MCL-CA & MCL-US & MCL-US & MCL-CA & MCL-US & NL-CA & MCL-US & MCL-CA & AL-US & SMCL-CA & AL-US & na \\
\hline Benchmark level & 1,000 & 6 & 10 & 1,000 & 4 & 1,000 & 5 & 50 & 1,300 & 300 & 15 & na \\
\hline [LT-MDL] or [SRL] & {$[2.2]$} & {$[0.027]$} & {$[0.03]$} & {$[0.07]$} & {$[0.006]$} & [3] & {$[0.016]$} & {$[0.07]$} & ${ }^{1}[2.1]$ & ${ }^{1}[6]$ & ${ }^{1}[0.82]$ & {$[0.22]$} \\
\hline \multicolumn{13}{|c|}{ San Gabriel study area (37 wells sampled) —Continued } \\
\hline HR-SG-31 & - & - & 0.12 & 52.2 & - & 74 & 0.029 & - & $\leq 0.74$ & 52.5 & $\leq 0.357$ & 0.58 \\
\hline HR-SG-32 & ${ }^{2} 1.8$ & 0.107 & 0.35 & 7.95 & - & 4 & - & 0.08 & $\leq 0.55$ & $* 5,970$ & $\leq 0.031$ & 6.22 \\
\hline HR-SG-33 & 2.2 & 0.029 & 0.92 & 78.2 & 0.008 & 449 & - & 2.2 & 10.6 & 74.3 & 1.60 & 5.57 \\
\hline HR-SGU-01 & - & 0.118 & 0.12 & 109 & 0.014 & 201 & 0.037 & 0.14 & 4.4 & - & 1.44 & 11.2 \\
\hline HR-SGU-02 & 3.0 & 0.143 & 0.05 & 8.02 & - & 23 & - & 0.15 & $\leq 1.0$ & - & $\leq 0.149$ & 2.18 \\
\hline HR-SGU-03 & - & 0.295 & 1.6 & 6.19 & - & 18 & - & 0.18 & - & $\leq 3.7$ & $\leq 0.016$ & 3.66 \\
\hline HR-SGU-04 & ${ }^{2} 2.1$ & 0.167 & $* 24.7$ & 28.4 & - & 4 & - & 0.09 & $\leq 0.88$ & 271 & 1.17 & 1.97 \\
\hline \multicolumn{13}{|c|}{ Peninsular Ranges study area (50 wells sampled) } \\
\hline HR-PR-01 & - & 0.052 & 1.7 & 204 & - & 58 & 0.055 & - & 3.8 & $\leq 5.7$ & 1.59 & 10.3 \\
\hline HR-PR-02 & 28.3 & - & 0.14 & 753 & - & 58 & 0.018 & - & 3.2 & 23.6 & $\leq 0.229$ & 29.0 \\
\hline HR-PR-03 & - & - & 0.08 & 1.65 & - & 3 & 0.017 & - & - & $* 475$ & $\leq 0.161$ & 10.4 \\
\hline HR-PR-04 & - & - & 0.13 & 73.2 & 0.008 & 19 & - & - & - & * 679 & $\leq 0.409$ & 25.2 \\
\hline HR-PR-05 & - & - & 0.49 & 160 & - & 21 & 0.021 & - & 2.6 & 6.0 & $\leq 0.394$ & 10.9 \\
\hline HR-PR-06 & 2.4 & - & 0.62 & 15.5 & 0.008 & 252 & 0.028 & - & $\leq 0.99$ & $\leq 5.8$ & $\leq 0.095$ & 46.5 \\
\hline HR-PR-07 & - & 0.340 & 4.0 & 44.1 & - & 163 & 0.103 & - & 6.8 & 14.3 & $\leq 0.105$ & 29.4 \\
\hline HR-PR-08 & - & 1.30 & 4.5 & 0.61 & - & 10 & - & - & $\leq 0.82$ & - & $\leq 0.084$ & 0.88 \\
\hline HR-PR-09 & - & - & 0.11 & 1.62 & - & 6 & - & 0.11 & - & 245 & $\leq 0.256$ & 3.60 \\
\hline HR-PR-10 & - & - & 0.31 & 48.1 & 0.155 & 10 & - & - & 0.58 & $* 4,040$ & - & 16.4 \\
\hline
\end{tabular}


Table 8. Trace elements detected in the samples collected for the Santa Cruz, San Gabriel, and Peninsular Ranges Hard Rock Aquifers (Hard Rock) study unit, March 2011 through March 2012, California GAMA Priority Basin Project.-Continued

[The five-digit U.S. Geological Survey (USGS) parameter code below the constituent name is used to uniquely identify a specific constituent or property. GAMA well identification number: HR-SC, Santa Cruz study area grid well; HR-SG, San Gabriel study area grid well; HR-SGU, San Gabriel study area understanding well; HR-PR, Peninsular Ranges study area grid well; HR-PRFP, Peninsular Ranges study area flow-path well; HR-PRU, Peninsular Ranges study area understanding well. Reporting levels, benchmark types, and benchmark levels as of July 6, 2012. Benchmark type: Maximum contaminant level benchmarks are listed as MCL-US when the MCL-US and MCL-CA are identical, and as MCL-CA when the MCL-CA is lower than the MCL-US or no MCL-US exists. Secondary maximum contaminant level benchmarks are listed as SMCL-CA when the SMCL-CA exists, and as MCL-US when the SMCL-CA does not exist. AL-US, U.S. Environmental Protection Agency (USEPA) action level; HAL-US, USEPA lifetime health advisory level; MCL-CA, California Department of Public Health (CDPH) maximum contaminant level; MCL-US, USEPA maximum contaminant level; NL-CA, CDPH notification level;

SMCL-CA, CDPH secondary maximum contaminant level. Other abbreviations: LT-MDL, long-term method detection level; SRL, study reporting level; $\mu \mathrm{g} / \mathrm{L}$, micrograms per liter; - , not detected; $\leq$, less than or equal to; *, value above benchmark level]

\begin{tabular}{|c|c|c|c|c|c|c|c|c|c|c|c|c|}
\hline $\begin{array}{c}\text { GAMA well } \\
\text { identification } \\
\text { number }\end{array}$ & $\begin{array}{c}\text { Aluminum } \\
(\mu \mathrm{g} / \mathrm{L}) \\
(01106)\end{array}$ & $\begin{array}{c}\text { Antimony } \\
(\mu \mathrm{g} / \mathrm{L}) \\
(01095) \\
\end{array}$ & $\begin{array}{c}\text { Arsenic } \\
(\mu \mathrm{g} / \mathrm{L}) \\
(01000)\end{array}$ & $\begin{array}{c}\text { Barium } \\
\text { ( } \mu \mathrm{g} / \mathrm{L}) \\
(01005)\end{array}$ & $\begin{array}{c}\text { Beryllium } \\
(\mu \mathrm{g} / \mathrm{L}) \\
(01010) \\
\end{array}$ & $\begin{array}{l}\text { Boron } \\
(\mu \mathrm{g} / \mathrm{L}) \\
(01020)\end{array}$ & $\begin{array}{c}\text { Cadmium } \\
(\mu \mathrm{g} / \mathrm{L}) \\
(01025)\end{array}$ & $\begin{array}{c}\text { Chromium } \\
(\mu \mathrm{g} / \mathrm{L}) \\
(01030)\end{array}$ & $\begin{array}{c}\text { Copper } \\
(\mu \mathrm{g} / \mathrm{L}) \\
(01040)\end{array}$ & $\begin{array}{c}\text { Iron } \\
\text { ( } \mu \mathrm{g} / \mathrm{L}) \\
(01046)\end{array}$ & $\begin{array}{c}\text { Lead } \\
(\mu \mathrm{g} / \mathrm{L}) \\
(01049)\end{array}$ & $\begin{array}{c}\text { Lithium } \\
(\mu \mathrm{g} / \mathrm{L}) \\
(01130)\end{array}$ \\
\hline Benchmark type & MCL-CA & MCL-US & MCL-US & MCL-CA & MCL-US & NL-CA & MCL-US & MCL-CA & AL-US & SMCL-CA & AL-US & na \\
\hline Benchmark level & 1,000 & 6 & 10 & 1,000 & 4 & 1,000 & 5 & 50 & 1,300 & 300 & 15 & na \\
\hline [LT-MDL] or [SRL] & [2.2] & {$[0.027]$} & {$[0.03]$} & {$[0.07]$} & {$[0.006]$} & [3] & {$[0.016]$} & {$[0.07]$} & ${ }^{1}[2.1]$ & ${ }^{1}[6]$ & ${ }^{1}[0.82]$ & {$[0.22]$} \\
\hline \multicolumn{13}{|c|}{ Peninsular Ranges study area (50 wells sampled)_Continued } \\
\hline HR-PR-11 & - & - & 0.89 & 5.76 & - & 35 & 0.032 & - & 0.68 & - & 1.01 & 65.2 \\
\hline HR-PR-12 & - & 0.033 & 0.44 & 44.4 & - & 103 & 0.019 & - & 3.1 & $\leq 4.9$ & $\leq 0.385$ & 7.02 \\
\hline HR-PR-13 & - & - & 0.71 & 149 & - & 170 & 0.043 & - & $\leq 0.53$ & $\leq 4.3$ & $\leq 0.341$ & 8.16 \\
\hline HR-PR-14 & - & - & 0.33 & 14.2 & - & 41 & 0.018 & 0.15 & 3.1 & - & $\leq 0.028$ & 4.39 \\
\hline HR-PR-15 & 3.1 & - & 0.13 & 45.9 & - & 4 & - & 0.11 & 7.5 & 12.3 & $\leq 0.378$ & 6.60 \\
\hline HR-PR-16 & - & 0.043 & 0.24 & 13.5 & 0.031 & 84 & - & - & - & * 1,220 & $\leq 0.182$ & 28.7 \\
\hline HR-PR-17 & ${ }^{2} 2.0$ & - & 0.25 & 60.1 & - & 55 & 0.047 & - & $\leq 0.89$ & $\leq 3.4$ & $\leq 0.107$ & 12.5 \\
\hline HR-PR-18 & - & 0.121 & 0.50 & 10.9 & 0.113 & 45 & 0.025 & - & - & $* 1,530$ & $\leq 0.120$ & 49.8 \\
\hline HR-PR-19 & - & 0.048 & 0.59 & 29.6 & - & 24 & 0.029 & - & - & 40.2 & $\leq 0.244$ & 16.2 \\
\hline HR-PR-20 & - & 0.182 & 2.0 & 18.5 & - & 15 & 0.033 & - & 3.1 & 21.7 & $\leq 0.635$ & 12.5 \\
\hline HR-PR-21 & - & 0.031 & 0.57 & 36.9 & - & 65 & 0.078 & - & 4.2 & 9.0 & $\leq 0.480$ & 39.1 \\
\hline HR-PR-22 & - & 0.044 & 0.51 & 5.72 & - & 42 & 0.016 & - & - & * 362 & $\leq 0.035$ & 15.2 \\
\hline HR-PR-23 & 3.6 & - & 6.8 & 61.4 & 0.022 & 155 & - & - & - & $* 1,700$ & 1.10 & 32.5 \\
\hline HR-PR-24 & 4.4 & - & 0.35 & 26.7 & - & 31 & 0.054 & 0.83 & 8.0 & 14.3 & 1.52 & 64.4 \\
\hline HR-PR-25 & - & - & 0.76 & 4.79 & - & 53 & - & 0.34 & - & - & $\leq 0.062$ & 1.33 \\
\hline HR-PR-26 3 & - & 0.063 & 0.20 & 32.5 & - & 181 & 0.597 & - & 2.5 & 43.6 & 0.872 & 2.64 \\
\hline HR-PR-27 & - & - & 0.05 & 17.1 & - & 10 & - & - & $\leq 1.2$ & - & $\leq 0.204$ & 14.6 \\
\hline HR-PR-28 & - & - & - & 16.1 & 0.049 & 49 & - & - & - & $* 1,770$ & - & 29.3 \\
\hline HR-PR-29 & 23.7 & - & 0.48 & 0.12 & - & 52 & 0.036 & - & - & 6.7 & $\leq 0.058$ & 2.86 \\
\hline HR-PR-30 & 5.8 & - & 0.18 & 29.9 & - & 9 & - & - & $\leq 0.94$ & - & $\leq 0.153$ & 23.7 \\
\hline
\end{tabular}

HR-PR-30 
Table 8. Trace elements detected in the samples collected for the Santa Cruz, San Gabriel, and Peninsular Ranges Hard Rock Aquifers (Hard Rock) study unit, March 2011 through March 2012, California GAMA Priority Basin Project.-Continued

[The five-digit U.S. Geological Survey (USGS) parameter code below the constituent name is used to uniquely identify a specific constituent or property. GAMA well identification number: HR-SC, Santa Cruz study area grid well; HR-SG, San Gabriel study area grid well; HR-SGU, San Gabriel study area understanding well; HR-PR, Peninsular Ranges study area grid well; HR-PRFP, Peninsular Ranges study area flow-path well; HR-PRU, Peninsular Ranges study area understanding well. Reporting levels, benchmark types, and benchmark levels as of July 6, 2012. Benchmark type: Maximum contaminant level benchmarks are listed as MCL-US when the MCL-US and MCL-CA are identical, and as MCL-CA when the MCL-CA is lower than the MCL-US or no MCL-US exists. Secondary maximum contaminant level benchmarks are listed as SMCL-CA when the SMCL-CA exists, and as MCL-US when the SMCL-CA does not exist. AL-US, U.S. Environmental Protection Agency (USEPA) action level; HAL-US, USEPA lifetime health advisory level; MCL-CA, California Department of Public Health (CDPH) maximum contaminant level; MCL-US, USEPA maximum contaminant level; NL-CA, CDPH notification level; SMCL-CA, CDPH secondary maximum contaminant level. Other abbreviations: LT-MDL, long-term method detection level; SRL, study reporting level; $\mu \mathrm{g} / \mathrm{L}$, micrograms per liter; - , not detected; $\leq$, less than or equal to; *, value above benchmark level]

\begin{tabular}{|c|c|c|c|c|c|c|c|c|c|c|c|c|}
\hline $\begin{array}{c}\text { GAMA well } \\
\text { identification } \\
\text { number }\end{array}$ & $\begin{array}{c}\text { Aluminum } \\
(\mu \mathrm{g} / \mathrm{L}) \\
(01106)\end{array}$ & $\begin{array}{c}\text { Antimony } \\
(\mu \mathrm{g} / \mathrm{L}) \\
(01095)\end{array}$ & $\begin{array}{c}\text { Arsenic } \\
(\mu \mathrm{g} / \mathrm{L}) \\
(01000)\end{array}$ & $\begin{array}{l}\text { Barium } \\
(\mu \mathrm{g} / \mathrm{L}) \\
(01005)\end{array}$ & $\begin{array}{c}\text { Beryllium } \\
(\mu \mathrm{g} / \mathrm{L}) \\
(01010)\end{array}$ & $\begin{array}{l}\text { Boron } \\
(\mu \mathrm{g} / \mathrm{L}) \\
(01020)\end{array}$ & $\begin{array}{c}\text { Cadmium } \\
(\mu \mathrm{g} / \mathrm{L}) \\
(01025)\end{array}$ & $\begin{array}{c}\text { Chromium } \\
(\mu \mathrm{g} / \mathrm{L}) \\
(01030)\end{array}$ & $\begin{array}{c}\text { Copper } \\
(\mu \mathrm{g} / \mathrm{L}) \\
(01040)\end{array}$ & $\begin{array}{c}\text { Iron } \\
(\mu \mathrm{g} / \mathrm{L}) \\
(01046)\end{array}$ & $\begin{array}{c}\text { Lead } \\
\text { ( } \mu \mathrm{g} / \mathrm{L}) \\
(01049)\end{array}$ & $\begin{array}{c}\text { Lithium } \\
(\mu \mathrm{g} / \mathrm{L}) \\
(01130)\end{array}$ \\
\hline Benchmark type & MCL-CA & MCL-US & MCL-US & MCL-CA & MCL-US & NL-CA & MCL-US & MCL-CA & AL-US & SMCL-CA & AL-US & na \\
\hline Benchmark level & 1,000 & 6 & 10 & 1,000 & 4 & 1,000 & 5 & 50 & 1,300 & 300 & 15 & na \\
\hline [LT-MDL] or [SRL] & [2.2] & {$[0.027]$} & {$[0.03]$} & {$[0.07]$} & {$[0.006]$} & [3] & {$[0.016]$} & {$[0.07]$} & ${ }^{1}[2.1]$ & ${ }^{1}[6]$ & ${ }^{1}[0.82]$ & {$[0.22]$} \\
\hline \multicolumn{13}{|c|}{ Peninsular Ranges study area (50 wells sampled)—Continued } \\
\hline HR-PR-31 & 6.0 & 0.034 & 0.84 & 16.7 & - & 19 & 0.020 & - & - & $\leq 4.6$ & $\leq 0.100$ & 4.41 \\
\hline HR-PR-32 & - & - & 0.54 & 11.7 & 0.008 & 43 & 0.028 & - & 6.6 & $\leq 3.4$ & $\leq 0.498$ & 6.18 \\
\hline HR-PR-33 & - & - & 0.15 & 218 & - & 71 & 0.027 & - & - & 10.3 & $\leq 0.061$ & 126 \\
\hline HR-PR-34 & - & 0.036 & 1.1 & 148 & - & 82 & - & - & $\leq 1.5$ & 11.3 & $\leq 0.145$ & 31.4 \\
\hline HR-PR-35 & - & - & 0.32 & 28.2 & - & 11 & - & - & - & - & $\leq 0.098$ & 46.9 \\
\hline HR-PR-36 & - & 0.029 & - & 3.03 & - & 6 & 0.017 & - & $\leq 1.7$ & 8.0 & 2.77 & 15.5 \\
\hline HR-PRFP-01 & - & - & 0.58 & 4.44 & 0.019 & 75 & - & - & - & $* 1,410$ & $\leq 0.098$ & 43.4 \\
\hline HR-PRFP-02 ${ }^{3}$ & - & - & 0.94 & 39.2 & - & 86 & - & - & 4.5 & 7.4 & 1.61 & 27.4 \\
\hline HR-PRFP-03 ${ }^{3}$ & - & - & 1.1 & 35.0 & - & 25 & - & - & - & 8.0 & $\leq 0.686$ & 31.3 \\
\hline HR-PRFP-04 ${ }^{3}$ & - & - & 0.96 & 11.9 & - & 94 & - & - & 11.3 & $\leq 4.1$ & 11.1 & 84.9 \\
\hline HR-PRFP-05 & - & - & 5.0 & 9.94 & 0.012 & 146 & 0.063 & - & - & 21.1 & $\leq 0.120$ & 34.2 \\
\hline HR-PRFP-06 & - & - & $* 13.7$ & 2.16 & 0.007 & 222 & 0.112 & - & - & 129 & $\leq 0.250$ & 22.9 \\
\hline HR-PRFP-07 & - & - & 0.11 & 3.83 & 0.012 & 30 & - & - & - & $* 481$ & $\leq 0.071$ & 11.5 \\
\hline HR-PRFP-08 & 14.0 & - & 0.06 & 2.37 & - & 104 & - & - & - & $\leq 3.9$ & - & 10.7 \\
\hline HR-PRFP-09 & - & 0.097 & 1.9 & 50.2 & - & 198 & 0.073 & 0.23 & 7.0 & - & 1.44 & 43.3 \\
\hline HR-PRFP-10 & - & - & 0.48 & 10.1 & - & 13 & 0.055 & - & 3.9 & 161 & 1.43 & 49.1 \\
\hline HR-PRFP-11 3 & - & 0.073 & 0.76 & 221 & 0.018 & 314 & - & 0.18 & 7.5 & 10.9 & 3.45 & 21.3 \\
\hline HR-PRFP-12 3 & - & - & 0.36 & 25.7 & - & 87 & - & 0.18 & - & $\leq 5.0$ & $\leq 0.104$ & 5.99 \\
\hline HR-PRFP-13 ${ }^{3}$ & - & 1.61 & 5.9 & 16.9 & 0.093 & 308 & 0.851 & - & 3.6 & 257 & $\leq 0.719$ & 147 \\
\hline HR-PRU-01 & 2.3 & - & 0.31 & 23.2 & - & 7 & - & - & $\leq 1.5$ & 12.5 & $\leq 0.484$ & 7.33 \\
\hline
\end{tabular}


Table 8. Trace elements detected in the samples collected for the Santa Cruz, San Gabriel, and Peninsular Ranges Hard Rock Aquifers (Hard Rock) study unit, March 2011

through March 2012, California GAMA Priority Basin Project.-Continued

[The five-digit U.S. Geological Survey (USGS) parameter code below the constituent name is used to uniquely identify a specific constituent or property. GAMA well identification number: HR-SC, Santa Cruz study area grid well; HR-SG, San Gabriel study area grid well; HR-SGU, San Gabriel study area understanding well; HR-PR, Peninsular Ranges study area grid well; HR-PRFP, Peninsular Ranges study area flow-path well; HR-PRU, Peninsular Ranges study area understanding well. Reporting levels, benchmark types, and benchmark levels as of July 6, 2012. Benchmark type: Maximum contaminant level benchmarks are listed as MCL-US when the MCL-US and MCL-CA are identical, and as MCL-CA when the MCL-CA is lower than the MCL-US or no MCL-US exists. Secondary maximum contaminant level benchmarks are listed as SMCL-CA when the SMCL-CA exists, and as MCL-US when the SMCL-CA does not exist. AL-US, U.S. Environmental Protection Agency (USEPA) action level; HAL-US, USEPA lifetime health advisory level; MCL-CA, California Department of Public Health (CDPH) maximum contaminant level; MCL-US, USEPA maximum contaminant level; NL-CA, CDPH notification level;

SMCL-CA, CDPH secondary maximum contaminant level. Other abbreviations: LT-MDL, long-term method detection level; SRL, study reporting level; $\mu \mathrm{g} / \mathrm{L}$, micrograms per liter; —, not detected; $\leq$, less than or equal to; *, value above benchmark level]

\begin{tabular}{|c|c|c|c|c|c|c|c|c|c|c|c|}
\hline $\begin{array}{c}\text { GAMA well } \\
\text { identification } \\
\text { number }\end{array}$ & $\begin{array}{c}\text { Manganese } \\
(\mu \mathrm{g} / \mathrm{L}) \\
(01056)\end{array}$ & $\begin{array}{c}\text { Molybdenum } \\
(\mu \mathrm{g} / \mathrm{L}) \\
(01060)\end{array}$ & $\begin{array}{l}\text { Nickel } \\
\text { ( } \mu \mathrm{g} / \mathrm{L}) \\
(01065)\end{array}$ & $\begin{array}{c}\text { Selenium } \\
(\mu \mathrm{g} / \mathrm{L}) \\
(01145)\end{array}$ & $\begin{array}{c}\text { Silver } \\
(\mu \mathrm{g} / \mathrm{L}) \\
(01075)\end{array}$ & $\begin{array}{c}\text { Strontium } \\
(\mu \mathrm{g} / \mathrm{L}) \\
(01080)\end{array}$ & $\begin{array}{c}\text { Thallium } \\
\text { ( } \mu \mathrm{g} / \mathrm{L}) \\
(01057)\end{array}$ & $\begin{array}{c}\text { Tungsten } \\
\text { ( } \mu \mathrm{g} / \mathrm{L}) \\
(01155)\end{array}$ & $\begin{array}{c}\text { Uranium } \\
(\mu \mathrm{g} / \mathrm{L}) \\
(22703)\end{array}$ & $\begin{array}{c}\text { Vanadium } \\
(\mu \mathrm{g} / \mathrm{L}) \\
(01085)\end{array}$ & $\begin{array}{c}\text { Zinc } \\
(\mu \mathrm{g} / \mathrm{L}) \\
(01090)\end{array}$ \\
\hline Benchmark type & SMCL-CA & HAL-US & MCL-CA & MCL-US & SMCL-CA & HAL-US & MCL-US & na & MCL-US & NL-CA & SMCL-CA \\
\hline Benchmark level & 50 & 40 & 100 & 50 & 100 & 4,000 & 2 & na & 30 & 50 & 5,000 \\
\hline [LT-MDL] or [SRL] & ${ }^{1}[0.66]$ & ${ }^{1}[0.023]$ & ${ }^{1}[0.21]$ & {$[0.03]$} & {$[0.005]$} & {$[0.2]$} & {$[0.010]$} & ${ }^{1}[0.023]$ & {$[0.004]$} & {$[0.08]$} & ${ }^{1}[6.2]$ \\
\hline \multicolumn{12}{|c|}{ Santa Cruz study area (25 wells sampled) } \\
\hline HR-SC-01 & - & 24.8 & $\leq 0.14$ & 0.26 & - & 40.7 & - & 0.806 & 0.104 & 5.5 & 10.3 \\
\hline HR-SC-02 & * 59.6 & 1.34 & 0.95 & 0.16 & - & 230 & - & - & 0.116 & 0.71 & 7.1 \\
\hline HR-SC-03 & * 469 & * 113 & 1.5 & 0.08 & - & 213 & - & - & 0.049 & 0.10 & $\leq 4.0$ \\
\hline HR-SC-04 & $* 93$ & 0.637 & 1.1 & 0.07 & - & 1,220 & 0.018 & $\leq 0.022$ & 0.170 & 0.09 & $\leq 4.5$ \\
\hline HR-SC-05 & * 204 & 0.724 & $\leq 0.09$ & - & - & 91.6 & - & $\leq 0.014$ & - & - & 8.1 \\
\hline HR-SC-06 & 3.32 & 0.091 & 0.24 & 0.22 & - & 295 & - & - & 0.074 & 0.21 & - \\
\hline HR-SC-07 & $* 83.9$ & 0.821 & 0.84 & - & - & 331 & - & - & 0.087 & 0.38 & - \\
\hline HR-SC-08 & * 561 & 0.113 & 2.1 & 0.24 & - & 302 & - & - & 0.006 & - & 18.0 \\
\hline HR-SC-09 & 2.89 & 0.735 & - & - & 0.005 & 351 & - & 4.25 & 0.063 & 0.14 & - \\
\hline HR-SC-10 & 9.29 & 0.430 & 0.45 & 0.22 & - & 162 & - & $\leq 0.010$ & 0.031 & 0.41 & $\leq 2.9$ \\
\hline HR-SC-11 & - & 0.482 & 0.57 & 0.34 & - & 432 & - & - & 0.107 & 0.39 & $\leq 3.2$ \\
\hline HR-SC-12 & 12.9 & 0.653 & 1.6 & 0.04 & - & 2,020 & - & - & 0.304 & 0.76 & 88.1 \\
\hline HR-SC-13 & 0.81 & 0.356 & 11.0 & 0.33 & - & 80.9 & - & $\leq 0.021$ & 0.016 & 1.2 & 48.6 \\
\hline HR-SC-14 & * 897 & 0.237 & 1.7 & 0.30 & - & 181 & - & - & 0.013 & 0.26 & 9.0 \\
\hline HR-SC-15 & 1.93 & 4.77 & - & 0.06 & - & 73.2 & 0.010 & 4.92 & 6.19 & 1.7 & - \\
\hline
\end{tabular}


Table 8. Trace elements detected in the samples collected for the Santa Cruz, San Gabriel, and Peninsular Ranges Hard Rock Aquifers (Hard Rock) study unit, March 2011 through March 2012, California GAMA Priority Basin Project.-Continued

[The five-digit U.S. Geological Survey (USGS) parameter code below the constituent name is used to uniquely identify a specific constituent or property. GAMA well identification number: HR-SC, Santa Cruz study area grid well; HR-SG, San Gabriel study area grid well; HR-SGU, San Gabriel study area understanding well; HR-PR, Peninsular Ranges study area grid well; HR-PRFP, Peninsular Ranges study area flow-path well; HR-PRU, Peninsular Ranges study area understanding well. Reporting levels, benchmark types, and benchmark levels as of July 6, 2012. Benchmark type: Maximum contaminant level benchmarks are listed as MCL-US when the MCL-US and MCL-CA are identical, and as MCL-CA when the MCL-CA is lower than the MCL-US or no MCL-US exists. Secondary maximum contaminant leve benchmarks are listed as SMCL-CA when the SMCL-CA exists, and as MCL-US when the SMCL-CA does not exist. AL-US, U.S. Environmental Protection Agency (USEPA) action level; HAL-US, USEPA lifetime health advisory level; MCL-CA, California Department of Public Health (CDPH) maximum contaminant level; MCL-US, USEPA maximum contaminant level; NL-CA, CDPH notification level; SMCL-CA, CDPH secondary maximum contaminant level. Other abbreviations: LT-MDL, long-term method detection level; SRL, study reporting level; $\mu \mathrm{g} / \mathrm{L}$, micrograms per liter; —, not detected; $\leq$, less than or equal to; *, value above benchmark level]

\begin{tabular}{|c|c|c|c|c|c|c|c|c|c|c|c|}
\hline $\begin{array}{c}\text { GAMA well } \\
\text { identification } \\
\text { number }\end{array}$ & $\begin{array}{c}\text { Manganese } \\
(\mu \mathrm{g} / \mathrm{L}) \\
(01056)\end{array}$ & $\begin{array}{c}\text { Molybdenum } \\
(\mu \mathrm{g} / \mathrm{L}) \\
(01060)\end{array}$ & $\begin{array}{c}\text { Nickel } \\
\text { ( } \mu \mathrm{g} / \mathrm{L}) \\
(01065)\end{array}$ & $\begin{array}{c}\text { Selenium } \\
(\mu \mathrm{g} / \mathrm{L}) \\
(01145) \\
\end{array}$ & $\begin{array}{c}\text { Silver } \\
(\mu \mathrm{g} / \mathrm{L}) \\
(01075)\end{array}$ & $\begin{array}{c}\text { Strontium } \\
(\mu \mathrm{g} / \mathrm{L}) \\
(01080)\end{array}$ & $\begin{array}{c}\text { Thallium } \\
\text { ( } \mu \mathrm{g} / \mathrm{L}) \\
(01057)\end{array}$ & $\begin{array}{c}\text { Tungsten } \\
(\mu \mathrm{g} / \mathrm{L}) \\
(01155)\end{array}$ & $\begin{array}{c}\text { Uranium } \\
\text { ( } \mu \mathrm{g} / \mathrm{L}) \\
(22703)\end{array}$ & $\begin{array}{c}\text { Vanadium } \\
(\mu \mathrm{g} / \mathrm{L}) \\
(01085)\end{array}$ & $\begin{array}{c}\text { Zinc } \\
(\mu \mathrm{g} / \mathrm{L}) \\
(01090)\end{array}$ \\
\hline Benchmark type & SMCL-CA & HAL-US & MCL-CA & MCL-US & SMCL-CA & HAL-US & MCL-US & na & MCL-US & NL-CA & SMCL-CA \\
\hline Benchmark level & 50 & 40 & 100 & 50 & 100 & 4,000 & 2 & na & 30 & 50 & 5,000 \\
\hline [LT-MDL] or [SRL] & ${ }^{1}[0.66]$ & ${ }^{1}[0.023]$ & ${ }^{1}[0.21]$ & {$[0.03]$} & {$[0.005]$} & {$[0.2]$} & {$[0.010]$} & $1[0.023]$ & {$[0.004]$} & {$[0.08]$} & ${ }^{1}[6.2]$ \\
\hline \multicolumn{12}{|c|}{ Santa Cruz study area ( 25 wells sampled)—Continued } \\
\hline HR-SC-16 & $\leq 0.34$ & 3.24 & $\leq 0.11$ & - & - & 111 & - & $\leq 0.016$ & 0.120 & 0.48 & 339 \\
\hline HR-SC-17 & $* 56.3$ & 0.271 & 5.6 & 0.22 & - & 70.4 & - & - & 0.032 & 0.22 & 18.0 \\
\hline HR-SC-18 & 30.2 & 4.90 & $\leq 0.14$ & - & - & 122 & - & 0.027 & - & - & 13.6 \\
\hline HR-SC-19 & * 199 & 1.77 & $\leq 0.11$ & - & - & 166 & 0.015 & 0.036 & - & 0.09 & - \\
\hline HR-SC-20 & 2.55 & 1.42 & 1.3 & 0.11 & - & 321 & - & - & 0.765 & 3.8 & 14.0 \\
\hline HR-SC-21 & $* 359$ & 5.03 & $\leq 0.09$ & - & - & 119 & - & 0.086 & 0.004 & - & - \\
\hline HR-SC-22 & * 528 & 0.109 & 1.3 & - & - & 568 & - & - & 0.054 & 0.10 & $\leq 2.0$ \\
\hline HR-SC-23 & * 62.5 & 0.291 & 0.25 & - & - & 1,900 & - & 0.079 & 0.031 & - & 14.5 \\
\hline HR-SC-24 & *396 & 4.12 & 6.2 & 1.8 & - & 957 & - & - & 8.61 & 0.44 & 6.4 \\
\hline HR-SC-25 & - & 0.298 & 2.6 & 0.75 & - & 69.3 & - & - & 0.006 & 0.78 & 15.7 \\
\hline \multicolumn{12}{|c|}{ San Gabriel study area (37 wells sampled) } \\
\hline HR-SG-01 & - & 6.85 & $\leq 0.16$ & 0.57 & - & 594 & - & - & 2.62 & 1.7 & $\leq 2.4$ \\
\hline HR-SG-02 & 1.52 & 7.26 & $\leq 0.13$ & 0.28 & - & 473 & - & - & 11.9 & 2.3 & - \\
\hline HR-SG-03 & $\leq 0.13$ & 1.74 & $\leq 0.18$ & 0.10 & - & 776 & - & $\leq 0.012$ & 0.495 & 3.5 & - \\
\hline HR-SG-04 & $\leq 0.14$ & 2.32 & 0.34 & 3.42 & - & 777 & - & 0.065 & 8.96 & 1.7 & - \\
\hline HR-SG-05 ${ }^{3}$ & $\leq 0.57$ & 8.10 & - & 0.60 & - & 829 & - & 0.077 & 7.24 & 1.0 & - \\
\hline HR-SG-06 & - & 4.66 & - & 0.41 & - & 192 & - & 0.043 & 10.3 & 1.3 & - \\
\hline HR-SG-07 & 7.12 & 7.19 & - & - & - & 328 & - & 0.028 & $* 66.0$ & - & 20.5 \\
\hline HR-SG-08 & * 219 & 1.75 & 0.43 & 0.05 & - & 1,010 & - & 0.099 & 0.373 & 2.5 & - \\
\hline HR-SG-09 & 3.66 & 5.65 & - & 0.64 & - & 73.3 & - & 2.23 & 5.49 & 9.9 & 9.8 \\
\hline HR-SG-10 & $\leq 0.54$ & 7.56 & $\leq 0.15$ & 0.48 & - & 308 & - & 0.046 & 29.3 & 0.97 & 7.3 \\
\hline
\end{tabular}


Table 8. Trace elements detected in the samples collected for the Santa Cruz, San Gabriel, and Peninsular Ranges Hard Rock Aquifers (Hard Rock) study unit, March 2011

through March 2012, California GAMA Priority Basin Project.-Continued

[The five-digit U.S. Geological Survey (USGS) parameter code below the constituent name is used to uniquely identify a specific constituent or property. GAMA well identification number: HR-SC, Santa Cruz study area grid well; HR-SG, San Gabriel study area grid well; HR-SGU, San Gabriel study area understanding well; HR-PR, Peninsular Ranges study area grid well; HR-PRFP, Peninsular Ranges study area flow-path well; HR-PRU, Peninsular Ranges study area understanding well. Reporting levels, benchmark types, and benchmark levels as of July 6, 2012. Benchmark type: Maximum contaminant level benchmarks are listed as MCL-US when the MCL-US and MCL-CA are identical, and as MCL-CA when the MCL-CA is lower than the MCL-US or no MCL-US exists. Secondary maximum contaminant level benchmarks are listed as SMCL-CA when the SMCL-CA exists, and as MCL-US when the SMCL-CA does not exist. AL-US, U.S. Environmental Protection Agency (USEPA) action level; HAL-US, USEPA lifetime health advisory level; MCL-CA, California Department of Public Health (CDPH) maximum contaminant level; MCL-US, USEPA maximum contaminant level; NL-CA, CDPH notification level;

SMCL-CA, CDPH secondary maximum contaminant level. Other abbreviations: LT-MDL, long-term method detection level; SRL, study reporting level; $\mu \mathrm{g} / \mathrm{L}$, micrograms per liter; -, not detected; $\leq$, less than or equal to; *, value above benchmark level]

\begin{tabular}{|c|c|c|c|c|c|c|c|c|c|c|c|}
\hline $\begin{array}{c}\text { GAMA well } \\
\text { identification } \\
\text { number }\end{array}$ & $\begin{array}{c}\text { Manganese } \\
(\mu \mathrm{g} / \mathrm{L}) \\
(01056)\end{array}$ & $\begin{array}{c}\text { Molybdenum } \\
(\mu \mathrm{g} / \mathrm{L}) \\
(01060)\end{array}$ & $\begin{array}{c}\text { Nickel } \\
\text { ( } \mu \mathrm{g} / \mathrm{L}) \\
(01065)\end{array}$ & $\begin{array}{c}\text { Selenium } \\
(\mu \mathrm{g} / \mathrm{L}) \\
(01145)\end{array}$ & $\begin{array}{c}\text { Silver } \\
(\mu \mathrm{g} / \mathrm{L}) \\
(01075)\end{array}$ & $\begin{array}{c}\text { Strontium } \\
(\mu \mathrm{g} / \mathrm{L}) \\
(01080)\end{array}$ & $\begin{array}{c}\text { Thallium } \\
\text { ( } \mu \mathrm{g} / \mathrm{L}) \\
(01057)\end{array}$ & $\begin{array}{c}\text { Tungsten } \\
(\mu \mathrm{g} / \mathrm{L}) \\
(01155)\end{array}$ & $\begin{array}{c}\text { Uranium } \\
\text { ( } \mu \mathrm{g} / \mathrm{L}) \\
(22703)\end{array}$ & $\begin{array}{c}\text { Vanadium } \\
(\mu \mathrm{g} / \mathrm{L}) \\
(01085)\end{array}$ & $\begin{array}{c}\text { Zinc } \\
\text { ( } \mu \mathrm{g} / \mathrm{L}) \\
(01090)\end{array}$ \\
\hline Benchmark type & SMCL-CA & HAL-US & MCL-CA & MCL-US & SMCL-CA & HAL-US & MCL-US & na & MCL-US & NL-CA & SMCL-CA \\
\hline Benchmark level & 50 & 40 & 100 & 50 & 100 & 4,000 & 2 & na & 30 & 50 & 5,000 \\
\hline [LT-MDL] or [SRL] & ${ }^{1}[0.66]$ & ${ }^{1}[0.023]$ & ${ }^{1}[0.21]$ & {$[0.03]$} & {$[0.005]$} & {$[0.2]$} & {$[0.010]$} & ${ }^{1}[0.023]$ & {$[0.004]$} & {$[0.08]$} & ${ }^{1}[6.2]$ \\
\hline \multicolumn{12}{|c|}{ San Gabriel study area (37 wells sampled)_Continued } \\
\hline HR-SG-11 & $\leq 0.33$ & 4.36 & $\leq 0.13$ & 0.05 & - & 275 & - & 0.384 & 16.0 & 1.5 & $\leq 1.5$ \\
\hline HR-SG-12 & - & 0.043 & - & 0.10 & - & 201 & - & 0.025 & 1.16 & 3.4 & $\leq 3.7$ \\
\hline HR-SG-13 & 3.34 & 1.19 & 1.1 & 0.19 & - & 637 & - & 0.137 & 0.976 & 0.18 & 8.3 \\
\hline HR-SG-14 & 40.5 & 0.596 & 0.56 & 0.08 & - & 495 & - & 0.037 & 0.584 & - & 105 \\
\hline HR-SG-15 & 4.54 & 2.01 & 0.40 & 0.16 & - & 355 & - & - & 1.40 & 0.17 & 109 \\
\hline HR-SG-16 & $\leq 0.22$ & 0.512 & - & 0.52 & - & 905 & - & - & 11.8 & 4.9 & 23.8 \\
\hline HR-SG-17 & - & 0.054 & - & - & 0.009 & 267 & - & $\leq 0.014$ & $* 36.8$ & 0.64 & 15.0 \\
\hline HR-SG-18 & $\leq 0.17$ & 2.70 & 0.29 & 0.12 & - & 540 & - & 0.090 & 1.32 & 3.9 & 9.1 \\
\hline HR-SG-19 & $\leq 0.49$ & 3.00 & $\leq 0.18$ & 1.9 & - & 326 & - & 0.864 & 2.19 & 0.92 & 18.4 \\
\hline HR-SG-20 & $\leq 0.29$ & 11.7 & $\leq 0.13$ & 1.1 & - & 1,350 & - & - & 3.62 & 0.28 & 13.5 \\
\hline HR-SG-21 & 2.64 & 0.225 & $\leq 0.18$ & 0.15 & - & 335 & - & - & 0.011 & 1.1 & 129 \\
\hline HR-SG-22 & $\leq 0.46$ & 13.9 & 0.22 & 0.07 & - & 380 & - & 0.034 & 3.05 & 2.1 & 12.9 \\
\hline HR-SG-23 & - & 3.81 & 0.27 & 0.15 & - & 176 & - & 0.419 & 5.37 & 2.5 & $\leq 2.7$ \\
\hline HR-SG-24 & $\leq 0.63$ & $* 83.2$ & $\leq 0.09$ & 0.21 & - & 481 & - & 2.57 & 4.06 & 1.4 & $\leq 4.9$ \\
\hline HR-SG-25 & - & 0.347 & - & 0.20 & 0.006 & 91.4 & - & $\leq 0.011$ & 4.99 & 8.2 & 9.5 \\
\hline HR-SG-26 & - & 3.54 & - & 0.25 & - & 534 & - & 0.224 & 1.67 & 0.81 & - \\
\hline HR-SG-27 & $\leq 0.29$ & 0.042 & $\leq 0.11$ & 0.09 & 0.005 & 167 & - & $\leq 0.020$ & 13.7 & 2.4 & 26.8 \\
\hline HR-SG-28 & - & 1.54 & $\leq 0.14$ & 0.38 & - & 200 & - & $\leq 0.014$ & 2.54 & 2.5 & $\leq 4.2$ \\
\hline HR-SG-29 & $* 55.6$ & 6.47 & 1.8 & - & - & 419 & - & 0.025 & 6.42 & - & 122 \\
\hline HR-SG-30 & - & 2.61 & $\leq 0.10$ & 0.25 & - & 385 & - & 0.158 & 1.88 & 0.44 & 21.1 \\
\hline
\end{tabular}


Table 8. Trace elements detected in the samples collected for the Santa Cruz, San Gabriel, and Peninsular Ranges Hard Rock Aquifers (Hard Rock) study unit, March 2011 through March 2012, California GAMA Priority Basin Project.-Continued

[The five-digit U.S. Geological Survey (USGS) parameter code below the constituent name is used to uniquely identify a specific constituent or property. GAMA well identification number: HR-SC, Santa Cruz study area grid well; HR-SG, San Gabriel study area grid well; HR-SGU, San Gabriel study area understanding well; HR-PR, Peninsular Ranges study area grid well; HR-PRFP, Peninsular Ranges study area flow-path well; HR-PRU, Peninsular Ranges study area understanding well. Reporting levels, benchmark types, and benchmark levels as of July 6, 2012. Benchmark type: Maximum contaminant level benchmarks are listed as MCL-US when the MCL-US and MCL-CA are identical, and as MCL-CA when the MCL-CA is lower than the MCL-US or no MCL-US exists. Secondary maximum contaminant level benchmarks are listed as SMCL-CA when the SMCL-CA exists, and as MCL-US when the SMCL-CA does not exist. AL-US, U.S. Environmental Protection Agency (USEPA) action level; HAL-US, USEPA lifetime health advisory level; MCL-CA, California Department of Public Health (CDPH) maximum contaminant level; MCL-US, USEPA maximum contaminant level; NL-CA, CDPH notification level; SMCL-CA, CDPH secondary maximum contaminant level. Other abbreviations: LT-MDL, long-term method detection level; SRL, study reporting level; $\mu \mathrm{g} / \mathrm{L}$, micrograms per liter; - , not detected; $\leq$, less than or equal to; *, value above benchmark level]

\begin{tabular}{|c|c|c|c|c|c|c|c|c|c|c|c|}
\hline $\begin{array}{c}\text { GAMA well } \\
\text { identification } \\
\text { number }\end{array}$ & $\begin{array}{c}\text { Manganese } \\
(\mu \mathrm{g} / \mathrm{L}) \\
(01056)\end{array}$ & $\begin{array}{c}\text { Molybdenum } \\
(\mu \mathrm{g} / \mathrm{L}) \\
(01060)\end{array}$ & $\begin{array}{c}\text { Nickel } \\
\text { ( } \mu \mathrm{g} / \mathrm{L}) \\
(01065)\end{array}$ & $\begin{array}{c}\text { Selenium } \\
(\mu \mathrm{g} / \mathrm{L}) \\
(01145)\end{array}$ & $\begin{array}{c}\text { Silver } \\
(\mu \mathrm{g} / \mathrm{L}) \\
(01075)\end{array}$ & $\begin{array}{c}\text { Strontium } \\
(\mu \mathrm{g} / \mathrm{L}) \\
(01080)\end{array}$ & $\begin{array}{c}\text { Thallium } \\
(\mu \mathrm{g} / \mathrm{L}) \\
(01057)\end{array}$ & $\begin{array}{c}\text { Tungsten } \\
\text { ( } \mu \mathrm{g} / \mathrm{L}) \\
(01155)\end{array}$ & $\begin{array}{c}\text { Uranium } \\
(\mu \mathrm{g} / \mathrm{L}) \\
(22703)\end{array}$ & $\begin{array}{c}\text { Vanadium } \\
(\mu \mathrm{g} / \mathrm{L}) \\
(01085)\end{array}$ & $\begin{array}{c}\text { Zinc } \\
(\mu \mathrm{g} / \mathrm{L}) \\
(01090)\end{array}$ \\
\hline Benchmark type & SMCL-CA & HAL-US & MCL-CA & MCL-US & SMCL-CA & HAL-US & MCL-US & na & MCL-US & NL-CA & SMCL-CA \\
\hline Benchmark level & 50 & 40 & 100 & 50 & 100 & 4,000 & 2 & na & 30 & 50 & 5,000 \\
\hline [LT-MDL] or [SRL] & ${ }^{1}[0.66]$ & ${ }^{1}[0.023]$ & ${ }^{1}[0.21]$ & {$[0.03]$} & {$[0.005]$} & {$[0.2]$} & {$[0.010]$} & ${ }^{1}[0.023]$ & {$[0.004]$} & {$[0.08]$} & ${ }^{1}[6.2]$ \\
\hline \multicolumn{12}{|c|}{ San Gabriel study area (37 wells sampled)_Continued } \\
\hline HR-SG-31 & $\leq 0.63$ & 9.19 & $\leq 0.13$ & 0.11 & - & 334 & - & $\leq 0.017$ & 2.47 & 2.6 & $\leq 2.4$ \\
\hline HR-SG-32 & * 93.4 & 1.62 & 0.61 & - & - & 214 & - & - & 0.714 & - & 32.8 \\
\hline HR-SG-33 & 6.52 & 4.46 & 0.65 & 6.2 & - & 466 & - & $\leq 0.015$ & 4.54 & 4.9 & 15.8 \\
\hline HR-SGU-01 & - & 11.9 & $\leq 0.17$ & 0.65 & 0.009 & 1,530 & - & 0.032 & 17.8 & 0.48 & 12.5 \\
\hline HR-SGU-02 & $\leq 0.16$ & 1.27 & - & 0.08 & - & 163 & - & $\leq 0.016$ & 7.99 & 3.2 & $\leq 1.6$ \\
\hline HR-SGU-03 & $\leq 0.21$ & 3.87 & - & 0.62 & - & 121 & - & 0.251 & 2.74 & 0.88 & - \\
\hline HR-SGU-04 & 2.46 & 2.04 & $\leq 0.16$ & 0.34 & - & 479 & - & - & 3.40 & 0.47 & 12.6 \\
\hline \multicolumn{12}{|c|}{ Peninsular Ranges study area ( 50 wells sampled) } \\
\hline HR-PR-01 & $* 290$ & 8.93 & 0.80 & 0.30 & 0.007 & 355 & - & 0.027 & 11.6 & 32.6 & $\leq 6.2$ \\
\hline HR-PR-02 & 15.9 & 2.09 & 0.39 & 0.40 & 0.012 & 536 & - & 0.037 & 0.733 & 3.8 & 44.1 \\
\hline HR-PR-03 & 30.5 & 5.98 & - & 0.16 & - & 68.2 & - & 0.096 & 0.028 & 0.21 & 35.3 \\
\hline HR-PR-04 & $* 102$ & 1.42 & $\leq 0.14$ & 0.14 & - & 109 & - & 0.179 & 0.159 & 0.09 & $\leq 6.1$ \\
\hline HR-PR-05 & $* 10.7$ & 5.49 & - & 0.05 & - & 112 & - & 0.622 & 0.466 & 4.2 & 7.9 \\
\hline HR-PR-06 & * 87.8 & 8.01 & 0.21 & 0.05 & - & 285 & - & 12.7 & 3.60 & 0.77 & $\leq 2.6$ \\
\hline HR-PR-07 & 5.21 & 20.4 & 1.7 & 0.97 & 0.019 & 718 & 0.014 & 0.942 & * 30.7 & 34.5 & 96.6 \\
\hline HR-PR-08 & - & 4.13 & 0.22 & 7.7 & - & 223 & - & 0.023 & 0.156 & 11.9 & $\leq 5.3$ \\
\hline HR-PR-09 & 17.7 & 0.652 & 0.30 & 0.18 & - & 141 & - & 0.965 & 0.568 & 0.54 & 660 \\
\hline HR-PR-10 & * 335 & 0.676 & 1.4 & - & - & 426 & - & 0.117 & 0.022 & - & $\leq 5.0$ \\
\hline
\end{tabular}


Table 8. Trace elements detected in the samples collected for the Santa Cruz, San Gabriel, and Peninsular Ranges Hard Rock Aquifers (Hard Rock) study unit, March 2011 through March 2012, California GAMA Priority Basin Project.-Continued

[The five-digit U.S. Geological Survey (USGS) parameter code below the constituent name is used to uniquely identify a specific constituent or property. GAMA well identification number: HR-SC, Santa Cruz study area grid well; HR-SG, San Gabriel study area grid well; HR-SGU, San Gabriel study area understanding well; HR-PR, Peninsular Ranges study area grid well; HR-PRFP, Peninsular Ranges study area flow-path well; HR-PRU, Peninsular Ranges study area understanding well. Reporting levels, benchmark types, and benchmark levels as of July 6, 2012. Benchmark type: Maximum contaminant level benchmarks are listed as MCL-US when the MCL-US and MCL-CA are identical, and as MCL-CA when the MCL-CA is lower than the MCL-US or no MCL-US exists. Secondary maximum contaminant leve benchmarks are listed as SMCL-CA when the SMCL-CA exists, and as MCL-US when the SMCL-CA does not exist. AL-US, U.S. Environmental Protection Agency (USEPA) action level; HAL-US, USEPA lifetime health advisory level; MCL-CA, California Department of Public Health (CDPH) maximum contaminant level; MCL-US, USEPA maximum contaminant level; NL-CA, CDPH notification level; SMCL-CA, CDPH secondary maximum contaminant level. Other abbreviations: LT-MDL, long-term method detection level; SRL, study reporting level; $\mu \mathrm{g} / \mathrm{L}$, micrograms per liter; - , not detected; $\leq$, less than or equal to; *, value above benchmark level]

\begin{tabular}{|c|c|c|c|c|c|c|c|c|c|c|c|}
\hline $\begin{array}{c}\text { GAMA well } \\
\text { identification } \\
\text { number }\end{array}$ & $\begin{array}{c}\text { Manganese } \\
(\mu \mathrm{g} / \mathrm{L}) \\
(01056)\end{array}$ & $\begin{array}{c}\text { Molybdenum } \\
(\mu \mathrm{g} / \mathrm{L}) \\
(01060)\end{array}$ & $\begin{array}{c}\text { Nickel } \\
\text { ( } \mu \mathrm{g} / \mathrm{L}) \\
(01065)\end{array}$ & $\begin{array}{c}\text { Selenium } \\
(\mu \mathrm{g} / \mathrm{L}) \\
(01145)\end{array}$ & $\begin{array}{c}\text { Silver } \\
(\mu \mathrm{g} / \mathrm{L}) \\
(01075)\end{array}$ & $\begin{array}{c}\text { Strontium } \\
(\mu \mathrm{g} / \mathrm{L}) \\
(01080)\end{array}$ & $\begin{array}{c}\text { Thallium } \\
\text { ( } \mu \mathrm{g} / \mathrm{L}) \\
(01057)\end{array}$ & $\begin{array}{c}\text { Tungsten } \\
(\mu \mathrm{g} / \mathrm{L}) \\
(01155)\end{array}$ & $\begin{array}{c}\text { Uranium } \\
\text { ( } \mu \mathrm{g} / \mathrm{L}) \\
(22703)\end{array}$ & $\begin{array}{c}\text { Vanadium } \\
(\mu \mathrm{g} / \mathrm{L}) \\
(01085)\end{array}$ & $\begin{array}{c}\text { Zinc } \\
(\mu \mathrm{g} / \mathrm{L}) \\
(01090)\end{array}$ \\
\hline Benchmark type & SMCL-CA & HAL-US & MCL-CA & MCL-US & SMCL-CA & HAL-US & MCL-US & na & MCL-US & NL-CA & SMCL-CA \\
\hline Benchmark level & 50 & 40 & 100 & 50 & 100 & 4,000 & 2 & na & 30 & 50 & 5,000 \\
\hline [LT-MDL] or [SRL] & ${ }^{1}[0.66]$ & ${ }^{1}[0.023]$ & ${ }^{1}[0.21]$ & {$[0.03]$} & {$[0.005]$} & {$[0.2]$} & {$[0.010]$} & ${ }^{1}[0.023]$ & {$[0.004]$} & {$[0.08]$} & ${ }^{1}[6.2]$ \\
\hline \multicolumn{12}{|c|}{ Peninsular Ranges study area (50 wells sampled) —Continued } \\
\hline HR-PR-11 & 18.5 & 6.38 & 0.25 & 0.19 & - & 240 & - & 0.127 & 0.890 & 0.23 & 91.9 \\
\hline HR-PR-12 & $\leq 0.15$ & 3.00 & 0.26 & 1.3 & 0.024 & 286 & - & $\leq 0.012$ & 0.724 & 18.1 & 13.6 \\
\hline HR-PR-13 & $\leq 0.57$ & 17.0 & 0.32 & 2.2 & - & 868 & - & 0.031 & 8.62 & 17.4 & - \\
\hline HR-PR-14 & - & 8.47 & 0.36 & 1.1 & 0.010 & 189 & - & 0.029 & 6.10 & 21.4 & $\leq 2.4$ \\
\hline HR-PR-15 & 0.94 & 1.21 & $\leq 0.13$ & 0.25 & - & 70.4 & - & - & 0.148 & 0.73 & 10.7 \\
\hline HR-PR-16 & * 207 & 3.13 & 0.50 & - & - & 338 & - & $\leq 0.018$ & 0.280 & 0.12 & $\leq 5.8$ \\
\hline HR-PR-17 & - & 20.3 & $\leq 0.11$ & 0.70 & 0.027 & 260 & - & 0.570 & * 34.8 & 6.8 & $\leq 2.8$ \\
\hline HR-PR-18 & * 690 & 4.90 & 0.23 & - & - & 294 & 0.011 & 0.996 & 28.8 & - & $\leq 2.4$ \\
\hline HR-PR-19 & 0.70 & 6.58 & 0.40 & 1.3 & - & 106 & - & 0.174 & 7.86 & 9.1 & 46.8 \\
\hline HR-PR-20 & $\leq 0.57$ & 4.3 & 0.43 & 0.33 & - & 259 & - & - & 1.58 & 6.3 & 26.3 \\
\hline HR-PR-21 & * 214 & 14.0 & 0.68 & 0.44 & - & 252 & 0.011 & 0.196 & * 66.6 & 2.0 & 24.1 \\
\hline HR-PR-22 & * 254 & 6.44 & 1.9 & 0.05 & - & 164 & - & 0.731 & $* 61.2$ & 2.2 & 16.8 \\
\hline HR-PR-23 & * 116 & 2.46 & 0.40 & - & - & 104 & 0.015 & 1.99 & 0.093 & - & 81.9 \\
\hline HR-PR-24 & - & 9.28 & 1.6 & 0.61 & 0.113 & 509 & - & - & 1.06 & 8.1 & 37.2 \\
\hline HR-PR-25 & - & 0.734 & 0.80 & 1.2 & 0.005 & 297 & - & 0.225 & 1.18 & 4.9 & - \\
\hline HR-PR-26 3 & 45.9 & 2.77 & 1.3 & 0.18 & 0.098 & 738 & - & - & 4.53 & 1.4 & 17.9 \\
\hline HR-PR-27 & - & 0.322 & 0.21 & 0.17 & 0.007 & 221 & - & - & 0.158 & 3.5 & $\leq 1.6$ \\
\hline HR-PR-28 & $* 161$ & 4.57 & 1.8 & - & - & 508 & - & 3.03 & 0.252 & 0.09 & 13.9 \\
\hline HR-PR-29 & $\leq 0.32$ & 16.6 & - & - & - & 21.7 & - & 0.857 & 0.586 & 0.16 & - \\
\hline HR-PR-30 & $\leq 0.14$ & 3.05 & 0.85 & 0.07 & - & 164 & - & $\leq 0.019$ & 5.14 & 1.6 & 59.1 \\
\hline
\end{tabular}


Table 8. Trace elements detected in the samples collected for the Santa Cruz, San Gabriel, and Peninsular Ranges Hard Rock Aquifers (Hard Rock) study unit, March 2011 through March 2012, California GAMA Priority Basin Project.-Continued

[The five-digit U.S. Geological Survey (USGS) parameter code below the constituent name is used to uniquely identify a specific constituent or property. GAMA well identification number: HR-SC, Santa Cruz study area grid well; HR-SG, San Gabriel study area grid well; HR-SGU, San Gabriel study area understanding well; HR-PR, Peninsular Ranges study area grid well; HR-PRFP, Peninsular Ranges study area flow-path well; HR-PRU, Peninsular Ranges study area understanding well. Reporting levels, benchmark types, and benchmark levels as of July 6, 2012. Benchmark type: Maximum contaminant level benchmarks are listed as MCL-US when the MCL-US and MCL-CA are identical, and as MCL-CA when the MCL-CA is lower than the MCL-US or no MCL-US exists. Secondary maximum contaminant level benchmarks are listed as SMCL-CA when the SMCL-CA exists, and as MCL-US when the SMCL-CA does not exist. AL-US, U.S. Environmental Protection Agency (USEPA) action level; HAL-US, USEPA lifetime health advisory level; MCL-CA, California Department of Public Health (CDPH) maximum contaminant level; MCL-US, USEPA maximum contaminant level; NL-CA, CDPH notification level; SMCL-CA, CDPH secondary maximum contaminant level. Other abbreviations: LT-MDL, long-term method detection level; SRL, study reporting level; $\mu \mathrm{g} / \mathrm{L}$, micrograms per liter; -, not detected; $\leq$, less than or equal to; *, value above benchmark level]

\begin{tabular}{|c|c|c|c|c|c|c|c|c|c|c|c|}
\hline $\begin{array}{c}\text { GAMA well } \\
\text { identification } \\
\text { number }\end{array}$ & $\begin{array}{c}\text { Manganese } \\
(\mu \mathrm{g} / \mathrm{L}) \\
(01056)\end{array}$ & $\begin{array}{c}\text { Molybdenum } \\
\text { ( } \mu \mathrm{g} / \mathrm{L}) \\
(01060)\end{array}$ & 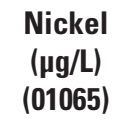 & $\begin{array}{c}\text { Selenium } \\
(\mu \mathrm{g} / \mathrm{L}) \\
(01145)\end{array}$ & $\begin{array}{c}\text { Silver } \\
(\mu \mathrm{g} / \mathrm{L}) \\
(01075)\end{array}$ & $\begin{array}{c}\text { Strontium } \\
(\mu \mathrm{g} / \mathrm{L}) \\
(01080)\end{array}$ & $\begin{array}{c}\text { Thallium } \\
\text { ( } \mu \mathrm{g} / \mathrm{L}) \\
(01057)\end{array}$ & $\begin{array}{c}\text { Tungsten } \\
\text { ( } \mu \mathrm{g} / \mathrm{L}) \\
(01155)\end{array}$ & $\begin{array}{c}\text { Uranium } \\
\text { ( } \mu \mathrm{g} / \mathrm{L}) \\
(22703)\end{array}$ & $\begin{array}{c}\text { Vanadium } \\
(\mu \mathrm{g} / \mathrm{L}) \\
(01085)\end{array}$ & $\begin{array}{c}\text { Zinc } \\
(\mu \mathrm{g} / \mathrm{L}) \\
(01090)\end{array}$ \\
\hline Benchmark type & SMCL-CA & HAL-US & MCL-CA & MCL-US & SMCL-CA & HAL-US & MCL-US & na & MCL-US & NL-CA & SMCL-CA \\
\hline Benchmark level & 50 & 40 & 100 & 50 & 100 & 4,000 & 2 & na & 30 & 50 & 5,000 \\
\hline [LT-MDL] or [SRL] & ${ }^{1}[0.66]$ & ${ }^{1}[0.023]$ & ${ }^{1}[0.21]$ & {$[0.03]$} & {$[0.005]$} & {$[0.2]$} & {$[0.010]$} & ${ }^{1}[0.023]$ & {$[0.004]$} & {$[0.08]$} & ${ }^{1}[6.2]$ \\
\hline \multicolumn{12}{|c|}{ Peninsular Ranges study area (50 wells sampled) —Continued } \\
\hline HR-PR-31 & 1.52 & 9.26 & 0.20 & 0.04 & - & 98.0 & - & 0.096 & 21.7 & 1.5 & $\leq 2.9$ \\
\hline HR-PR-32 & 3.00 & 5.28 & 1.8 & 0.07 & - & 1,220 & - & 0.054 & 0.796 & 0.94 & 14.2 \\
\hline HR-PR-33 & 28.4 & 0.359 & 0.37 & 0.84 & 0.015 & 492 & - & 0.030 & 2.85 & 2.3 & 9.7 \\
\hline HR-PR-34 & $\leq 0.21$ & 1.93 & 0.49 & 0.96 & - & 763 & - & $\leq 0.014$ & 4.82 & 5.5 & $\leq 5.7$ \\
\hline HR-PR-35 & $\leq 0.33$ & 1.45 & $\leq 0.14$ & 0.26 & - & 197 & - & $\leq 0.012$ & 1.26 & 3.5 & 12.5 \\
\hline HR-PR-36 & * 376 & 0.119 & 0.31 & - & - & 107 & - & - & 2.13 & 0.37 & 4,530 \\
\hline HR-PRFP-01 & * 226 & 1.58 & $\leq 0.09$ & - & - & 137 & 0.054 & 4.57 & 0.242 & - & $\leq 2.8$ \\
\hline HR-PRFP-02 3 & 0.90 & 7.12 & - & 4.2 & - & 179 & - & - & 12.0 & 14.7 & 938 \\
\hline HR-PRFP-03 ${ }^{3}$ & $* 136$ & 0.369 & - & 0.29 & - & 152 & - & 0.952 & 1.47 & 0.88 & 315 \\
\hline HR-PRFP-04 ${ }^{3}$ & 1.09 & 5.95 & 2.4 & 6.9 & - & 152 & - & 0.190 & $* 35.6$ & 4.5 & 248 \\
\hline HR-PRFP-05 & * 129 & 11.6 & 0.95 & 0.09 & 0.014 & 136 & 0.010 & 1.26 & $* 35.8$ & 1.8 & 45.4 \\
\hline HR-PRFP-06 & * 160 & 18.7 & 0.33 & 0.04 & - & 59.6 & - & 3.30 & 17.8 & 0.26 & 10.8 \\
\hline HR-PRFP-07 & * 107 & 12.3 & $\leq 0.12$ & - & - & 114 & - & 2.65 & 0.283 & - & 28.5 \\
\hline HR-PRFP-08 & 2.73 & 1.71 & - & - & - & 10.9 & - & 17.2 & 0.445 & 0.29 & $\leq 1.5$ \\
\hline HR-PRFP-09 & * 50.6 & 16.8 & 0.31 & 2.8 & 0.022 & 332 & 0.013 & 0.090 & $* 45.2$ & 10.3 & 12.8 \\
\hline
\end{tabular}


Table 8. Trace elements detected in the samples collected for the Santa Cruz, San Gabriel, and Peninsular Ranges Hard Rock Aquifers (Hard Rock) study unit, March 2011 through March 2012, California GAMA Priority Basin Project.-Continued

[The five-digit U.S. Geological Survey (USGS) parameter code below the constituent name is used to uniquely identify a specific constituent or property. GAMA well identification number: HR-SC, Santa Cruz study area grid well; HR-SG, San Gabriel study area grid well; HR-SGU, San Gabriel study area understanding well; HR-PR, Peninsular Ranges study area grid well; HR-PRFP, Peninsular Ranges study area flow-path well; HR-PRU, Peninsular Ranges study area understanding well. Reporting levels, benchmark types, and benchmark levels as of July 6, 2012. Benchmark type: Maximum contaminant level benchmarks are listed as MCL-US when the MCL-US and MCL-CA are identical, and as MCL-CA when the MCL-CA is lower than the MCL-US or no MCL-US exists. Secondary maximum contaminant level benchmarks are listed as SMCL-CA when the SMCL-CA exists, and as MCL-US when the SMCL-CA does not exist. AL-US, U.S. Environmental Protection Agency (USEPA) action level; HAL-US, USEPA lifetime health advisory level; MCL-CA, California Department of Public Health (CDPH) maximum contaminant level; MCL-US, USEPA maximum contaminant level; NL-CA, CDPH notification level;

SMCL-CA, CDPH secondary maximum contaminant level. Other abbreviations: LT-MDL, long-term method detection level; SRL, study reporting level; $\mu \mathrm{g} / \mathrm{L}$, micrograms per liter; —, not detected; $\leq$, less than or equal to; *, value above benchmark level]

\begin{tabular}{|c|c|c|c|c|c|c|c|c|c|c|c|}
\hline $\begin{array}{c}\text { GAMA well } \\
\text { identification } \\
\text { number }\end{array}$ & $\begin{array}{c}\text { Manganese } \\
(\mu \mathrm{g} / \mathrm{L}) \\
(01056)\end{array}$ & $\begin{array}{c}\text { Molybdenum } \\
(\mu \mathrm{g} / \mathrm{L}) \\
(01060) \\
\end{array}$ & $\begin{array}{c}\text { Nickel } \\
\text { ( } \mu \mathrm{g} / \mathrm{L}) \\
(01065)\end{array}$ & $\begin{array}{c}\text { Selenium } \\
(\mu \mathrm{g} / \mathrm{L}) \\
(01145) \\
\end{array}$ & $\begin{array}{c}\text { Silver } \\
(\mu \mathrm{g} / \mathrm{L}) \\
(01075) \\
\end{array}$ & $\begin{array}{c}\text { Strontium } \\
(\mu \mathrm{g} / \mathrm{L}) \\
(01080) \\
\end{array}$ & $\begin{array}{c}\text { Thallium } \\
\text { ( } \mu \mathrm{g} / \mathrm{L}) \\
(01057) \\
\end{array}$ & $\begin{array}{c}\text { Tungsten } \\
(\mu \mathrm{g} / \mathrm{L}) \\
(01155) \\
\end{array}$ & $\begin{array}{c}\text { Uranium } \\
(\mu \mathrm{g} / \mathrm{L}) \\
(22703)\end{array}$ & $\begin{array}{c}\text { Vanadium } \\
(\mu \mathrm{g} / \mathrm{L}) \\
(01085) \\
\end{array}$ & $\begin{array}{c}\text { Zinc } \\
\text { ( } \mu \mathrm{g} / \mathrm{L}) \\
(01090)\end{array}$ \\
\hline Benchmark type & SMCL-CA & HAL-US & MCL-CA & MCL-US & SMCL-CA & HAL-US & MCL-US & na & MCL-US & NL-CA & SMCL-CA \\
\hline Benchmark level & 50 & 40 & 100 & 50 & 100 & 4,000 & 2 & na & 30 & 50 & 5,000 \\
\hline [LT-MDL] or [SRL] & ${ }^{1}[0.66]$ & ${ }^{1}[0.023]$ & ${ }^{1}[0.21]$ & {$[0.03]$} & {$[0.005]$} & {$[0.2]$} & {$[0.010]$} & ${ }^{1}[0.023]$ & {$[0.004]$} & {$[0.08]$} & ${ }^{1}[6.2]$ \\
\hline \multicolumn{12}{|c|}{ Peninsular Ranges study area (50 wells sampled)—Continued } \\
\hline HR-PRFP-10 & $* 337$ & 2.91 & 0.32 & 0.07 & - & 112 & 0.016 & 0.036 & $* 286$ & 0.53 & 29.4 \\
\hline HR-PRFP-11 3 & 7.31 & 0.458 & 2.4 & 3.8 & 0.348 & $* 4,050$ & - & - & * 64.6 & 8.3 & 14.2 \\
\hline HR-PRFP-12 3 & $\leq 0.30$ & 6.56 & 0.61 & 1.2 & 0.071 & 600 & 0.037 & 0.340 & 8.28 & 17.9 & $\leq 3.4$ \\
\hline HR-PRFP-13 ${ }^{3}$ & $* 425$ & 6.05 & 4.4 & 4.5 & 0.027 & 741 & - & 0.042 & * 48.9 & 0.67 & 295 \\
\hline HR-PRU-01 & 1.25 & 3.92 & - & 0.04 & - & 47.0 & - & - & 0.305 & 0.29 & $\leq 3.1$ \\
\hline
\end{tabular}

${ }^{1}$ SRL defined based on examination of quality-control results from October 2009 through March 2013 (Davis and others, 2014). All results for cobalt were censored from this dataset and coded in the USGS National Water Information System (NWIS) database as "reviewed and rejected." All detections of aluminum, copper, iron, lead, manganese, molybdenum, nickel, tungsten, and zinc at concentrations less than the respective SRL are reported as greater than or equal to $(\leq)$ the value reported by the laboratory. In the NWIS database, results are accompanied with the following comment: Result is $<$ or $=$ reported value, based on quality-control data (Davis and others, 2014).

${ }^{2}$ Value is below the highest LT-MDL used by the NWQL during the time in which samples were analyzed for the Hard Rock study unit.

${ }^{3}$ LT-MDLs reported by the NWQL were raised due to dilution during analyses for all trace elements for the following Hard Rock samples: HR-SG-05 (by factor of 3); HR-PR-26 (by factor of 2); HR-PRFP-02, 03, and 04 (by factor of 5); and HR-PRFP-11, 12, and 13 (by factor of 2). 
Table 9. Nutrients detected in samples collected for the Santa Cruz, San Gabriel, and Peninsular Ranges Hard Rock Aquifers (Hard Rock) study unit, March 2011 through March 2012, California GAMA Priority Basin Project.

[The five-digit U.S. Geological Survey (USGS) parameter code below the constituent name is used to uniquely identify a specific constituent or property. Samples from all 112 wells were analyzed, but only samples with detections of at least one constituent are listed. GAMA well identification number: HR-SC, Santa Cruz study area grid well; HR-SG, San Gabriel study area grid well; HR-SGU, San Gabriel study area understanding well; HR-PR, Peninsular Ranges study area grid well; HR-PRFP, Peninsular Ranges study area flow-path well; HR-PRU, Peninsular Ranges study area understanding well. Reporting level, benchmark type, and benchmark level as of July 6, 2012. Benchmark type: Maximum contaminant level benchmarks are listed as MCL-US when the MCL-US and MCL-CA are identical, and as MCL-CA when the MCL-CA is lower than the MCL-US or no MCL-US exists. HAL-US, U.S. Environmental Protection Agency (USEPA) lifetime health advisory level; MCL-US, USEPA maximum contaminant level. Other abbreviations: SRL, study reporting level; IRL, interim reporting level; LT-MDL, long-term method detection level; mg/L, milligrams per liter; *, value above benchmark level; na, not available; —, not detected; $\leq$, less than or equal to]

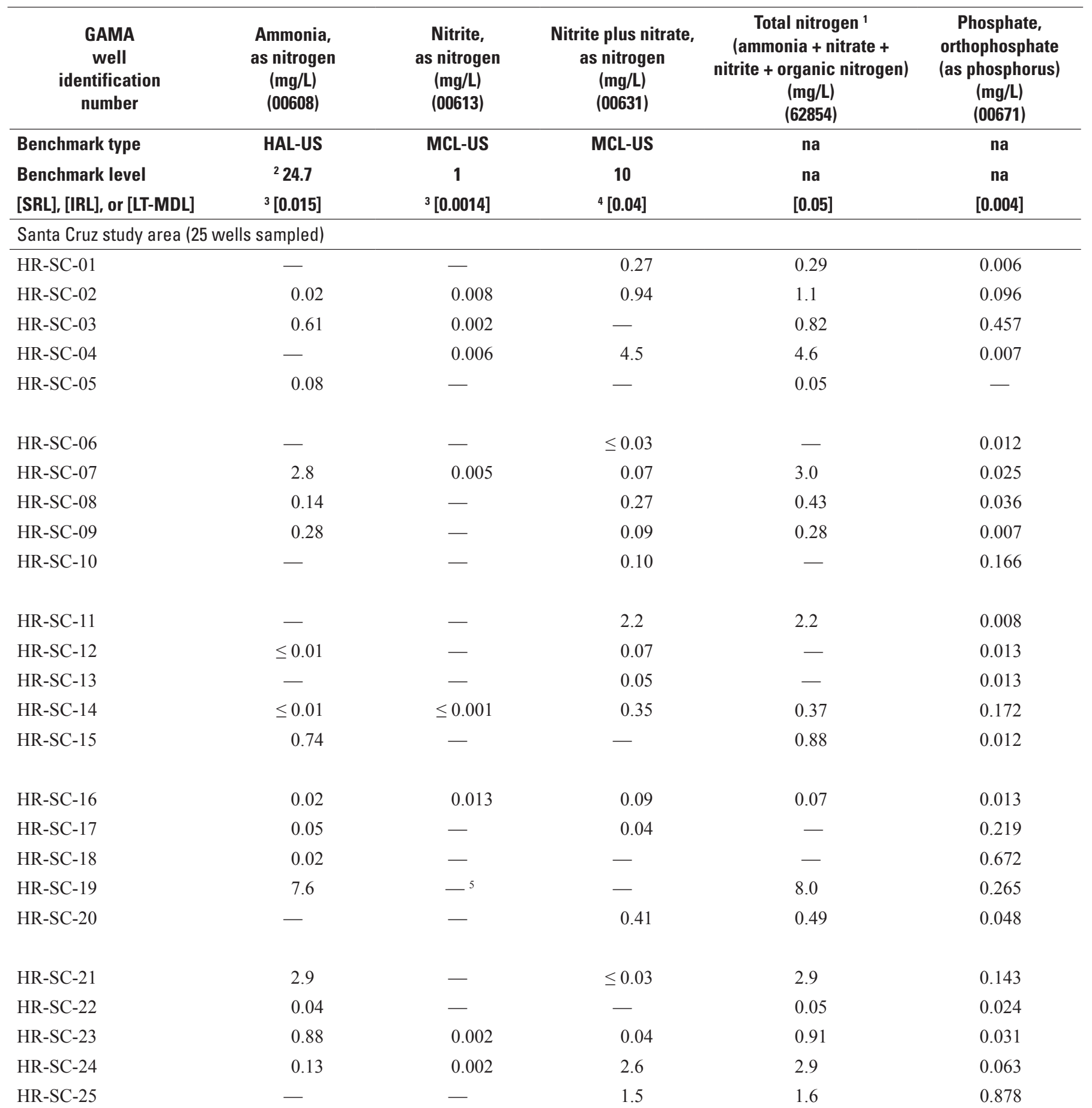


Table 9. Nutrients detected in samples collected for the Santa Cruz, San Gabriel, and Peninsular Ranges Hard Rock Aquifers (Hard Rock) study unit, March 2011 through March 2012, California GAMA Priority Basin Project.—Continued

[The five-digit U.S. Geological Survey (USGS) parameter code below the constituent name is used to uniquely identify a specific constituent or property. Samples from all 112 wells were analyzed, but only samples with detections of at least one constituent are listed. GAMA well identification number: HR-SC, Santa Cruz study area grid well; HR-SG, San Gabriel study area grid well; HR-SGU, San Gabriel study area understanding well; HR-PR, Peninsular Ranges study area grid well; HR-PRFP, Peninsular Ranges study area flow-path well; HR-PRU, Peninsular Ranges study area understanding well. Reporting level, benchmark type, and benchmark level as of July 6, 2012. Benchmark type: Maximum contaminant level benchmarks are listed as MCL-US when the MCL-US and MCL-CA are identical, and as MCL-CA when the MCL-CA is lower than the MCL-US or no MCL-US exists. HAL-US, U.S. Environmental Protection Agency (USEPA) lifetime health advisory level; MCL-US, USEPA maximum contaminant level. Other abbreviations: SRL, study reporting level; IRL, interim reporting level; LT-MDL, long-term method detection level; mg/L, milligrams per liter; *, value above benchmark level; na, not available; —, not detected; $\leq$, less than or equal to]

\begin{tabular}{|c|c|c|c|c|c|}
\hline $\begin{array}{c}\text { GAMA } \\
\text { well } \\
\text { identification } \\
\text { number }\end{array}$ & $\begin{array}{l}\text { Ammonia, } \\
\text { as nitrogen } \\
(\mathrm{mg} / \mathrm{L}) \\
(00608)\end{array}$ & $\begin{array}{c}\text { Nitrite, } \\
\text { as nitrogen } \\
(\mathrm{mg} / \mathrm{L}) \\
(00613)\end{array}$ & $\begin{array}{c}\text { Nitrite plus nitrate, } \\
\text { as nitrogen } \\
(\mathrm{mg} / \mathrm{L}) \\
(\mathbf{0 0 6 3 1})\end{array}$ & $\begin{array}{c}\text { Total nitrogen }{ }^{1} \\
\text { (ammonia + nitrate + } \\
\text { nitrite + organic nitrogen) } \\
\text { (mg/L) } \\
(62854)\end{array}$ & $\begin{array}{c}\text { Phosphate, } \\
\text { orthophosphate } \\
\text { (as phosphorus) } \\
\text { (mg/L) } \\
\text { (00671) }\end{array}$ \\
\hline Benchmark type & HAL-US & MCL-US & MCL-US & na & na \\
\hline Benchmark level & ${ }^{2} 24.7$ & 1 & 10 & na & na \\
\hline \multicolumn{6}{|c|}{ San Gabriel study area (37 wells sampled) ${ }^{6}$} \\
\hline HR-SG-01 & $\leq 0.01$ & - & 0.25 & 0.25 & 0.027 \\
\hline HR-SG-02 & $\leq 0.01$ & $\leq 0.001$ & 0.32 & 0.30 & 0.027 \\
\hline HR-SG-03 & $\leq 0.01$ & $\leq 0.001$ & 0.47 & 0.51 & 0.058 \\
\hline HR-SG-04 & $\leq 0.01$ & - & 6.0 & 6.2 & 0.041 \\
\hline HR-SG-08 & 0.02 & $\leq 0.001$ & - & 0.08 & 0.115 \\
\hline HR-SG-09 & 0.07 & 0.003 & 1.2 & 1.25 & 0.034 \\
\hline HR-SG-10 & - & - & 3.5 & 3.5 & 0.010 \\
\hline HR-SG-11 & - & - & $\leq 0.02$ & - & 0.006 \\
\hline HR-SG-12 & - & - & 0.04 & - & 0.014 \\
\hline HR-SG-13 & - & $\leq 0.001$ & 0.11 & 0.09 & 0.012 \\
\hline HR-SG-14 & - & 0.002 & 0.16 & 0.16 & 0.013 \\
\hline HR-SG-20 & - & - & 0.38 & 0.35 & 0.009 \\
\hline HR-SG-21 & - & $\leq 0.001$ & 0.18 & 0.15 & 0.023 \\
\hline HR-SG-22 & - & - & 0.13 & 0.17 & 0.045 \\
\hline HR-SG-23 & - & - & 0.48 & 0.48 & 0.015 \\
\hline HR-SG-24 & - & - & 0.22 & 0.21 & 0.012 \\
\hline HR-SG-25 & - & - & 1.3 & 1.2 & 0.044 \\
\hline HR-SG-26 & - & - & 0.38 & 0.37 & 0.012 \\
\hline
\end{tabular}


Table 9. Nutrients detected in samples collected for the Santa Cruz, San Gabriel, and Peninsular Ranges Hard Rock Aquifers (Hard Rock) study unit, March 2011 through March 2012, California GAMA Priority Basin Project._Continued

[The five-digit U.S. Geological Survey (USGS) parameter code below the constituent name is used to uniquely identify a specific constituent or property. Samples from all 112 wells were analyzed, but only samples with detections of at least one constituent are listed. GAMA well identification number: HR-SC, Santa Cruz study area grid well; HR-SG, San Gabriel study area grid well; HR-SGU, San Gabriel study area understanding well; HR-PR, Peninsular Ranges study area grid well; HR-PRFP, Peninsular Ranges study area flow-path well; HR-PRU, Peninsular Ranges study area understanding well. Reporting level, benchmark type, and benchmark level as of July 6, 2012. Benchmark type: Maximum contaminant level benchmarks are listed as MCL-US when the MCL-US and MCL-CA are identical, and as MCL-CA when the MCL-CA is lower than the MCL-US or no MCL-US exists. HAL-US, U.S. Environmental Protection Agency (USEPA) lifetime health advisory level; MCL-US, USEPA maximum contaminant level. Other abbreviations: SRL, study reporting level; IRL, interim reporting level; LT-MDL, long-term method detection level; mg/L, milligrams per liter; *, value above benchmark level; na, not available; —, not detected; $\leq$, less than or equal to]

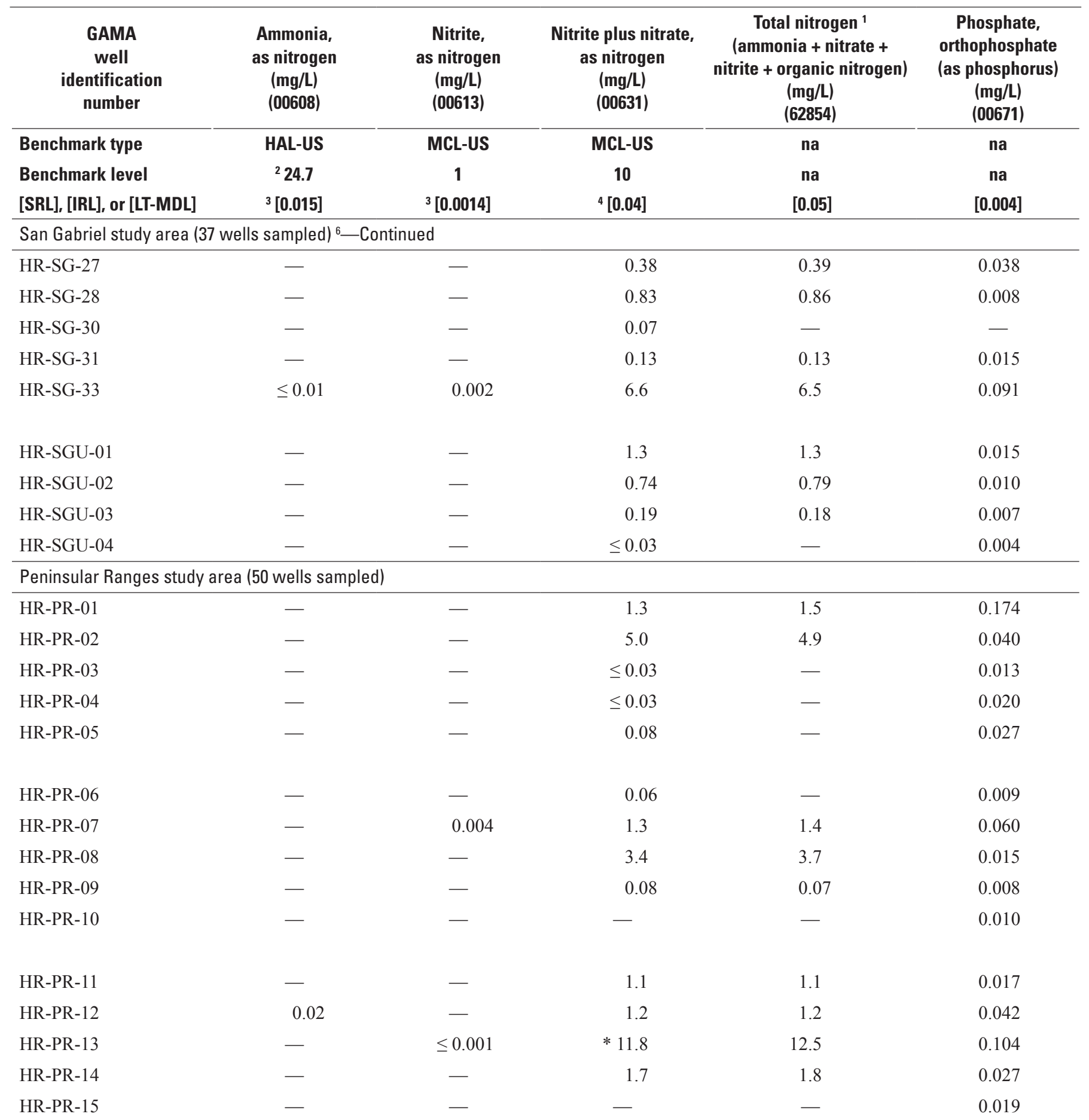


Table 9. Nutrients detected in samples collected for the Santa Cruz, San Gabriel, and Peninsular Ranges Hard Rock Aquifers (Hard Rock) study unit, March 2011 through March 2012, California GAMA Priority Basin Project.—Continued

[The five-digit U.S. Geological Survey (USGS) parameter code below the constituent name is used to uniquely identify a specific constituent or property. Samples from all 112 wells were analyzed, but only samples with detections of at least one constituent are listed. GAMA well identification number: HR-SC, Santa Cruz study area grid well; HR-SG, San Gabriel study area grid well; HR-SGU, San Gabriel study area understanding well; HR-PR, Peninsular Ranges study area grid well; HR-PRFP, Peninsular Ranges study area flow-path well; HR-PRU, Peninsular Ranges study area understanding well. Reporting level, benchmark type, and benchmark level as of July 6, 2012. Benchmark type: Maximum contaminant level benchmarks are listed as MCL-US when the MCL-US and MCL-CA are identical, and as MCL-CA when the MCL-CA is lower than the MCL-US or no MCL-US exists. HAL-US, U.S. Environmental Protection Agency (USEPA) lifetime health advisory level; MCL-US, USEPA maximum contaminant level. Other abbreviations: SRL, study reporting level; IRL, interim reporting level; LT-MDL, long-term method detection level; mg/L, milligrams per liter; *, value above benchmark level; na, not available; —, not detected; $\leq$, less than or equal to]

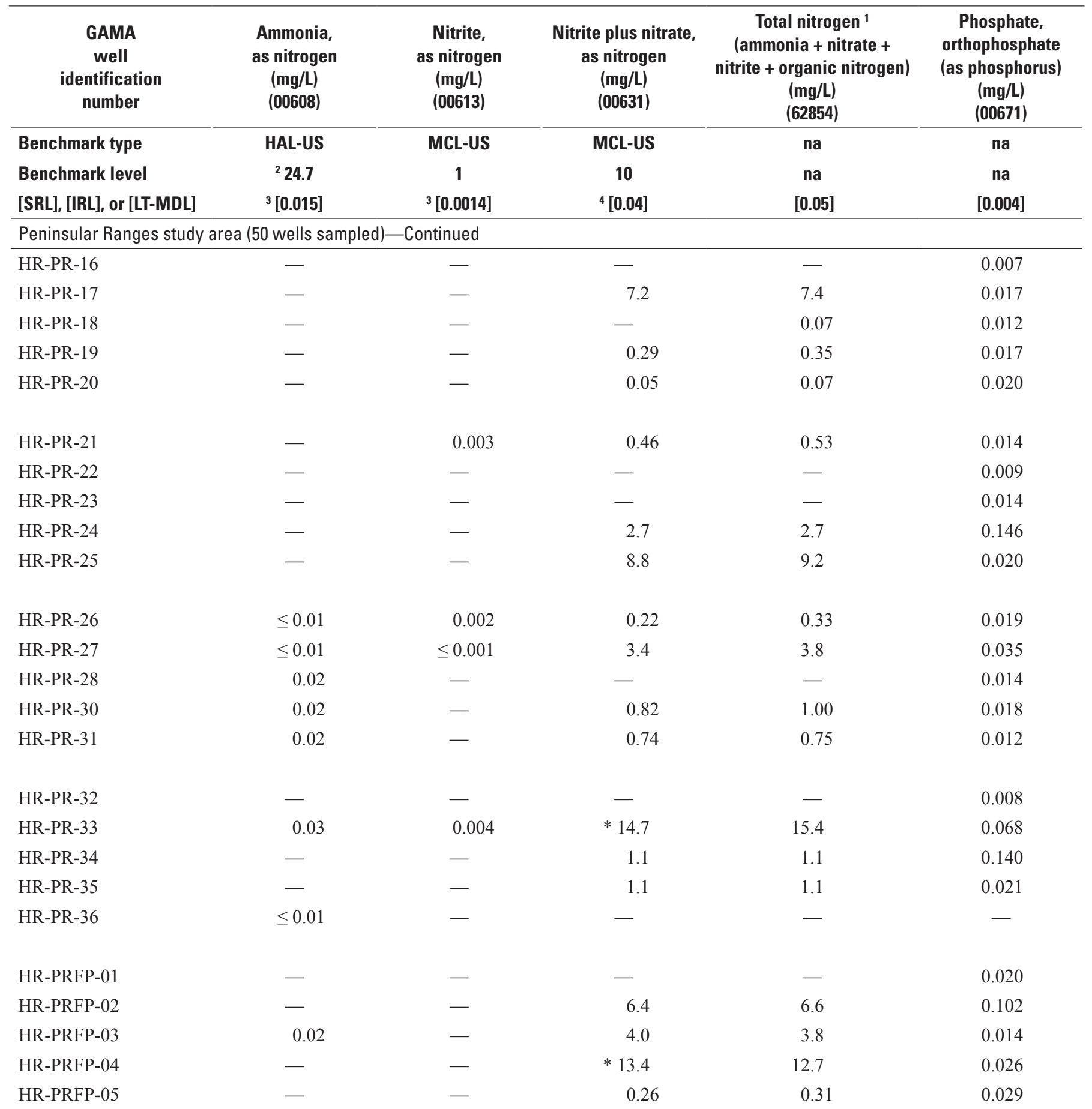


Table 9. Nutrients detected in samples collected for the Santa Cruz, San Gabriel, and Peninsular Ranges Hard Rock Aquifers (Hard Rock) study unit, March 2011 through March 2012, California GAMA Priority Basin Project.—Continued

[The five-digit U.S. Geological Survey (USGS) parameter code below the constituent name is used to uniquely identify a specific constituent or property. Samples from all 112 wells were analyzed, but only samples with detections of at least one constituent are listed. GAMA well identification number: HR-SC, Santa Cruz study area grid well; HR-SG, San Gabriel study area grid well; HR-SGU, San Gabriel study area understanding well; HR-PR, Peninsular Ranges study area grid well; HR-PRFP, Peninsular Ranges study area flow-path well; HR-PRU, Peninsular Ranges study area understanding well. Reporting level, benchmark type, and benchmark level as of July 6, 2012. Benchmark type: Maximum contaminant level benchmarks are listed as MCL-US when the MCL-US and MCL-CA are identical, and as MCL-CA when the MCL-CA is lower than the MCL-US or no MCL-US exists. HAL-US, U.S. Environmental Protection Agency (USEPA) lifetime health advisory level; MCL-US, USEPA maximum contaminant level. Other abbreviations: SRL, study reporting level; IRL, interim reporting level; LT-MDL, long-term method detection level; mg/L, milligrams per liter; *, value above benchmark level; na, not available; —, not detected; $\leq$, less than or equal to]

\begin{tabular}{|c|c|c|c|c|c|}
\hline $\begin{array}{c}\text { GAMA } \\
\text { well } \\
\text { identification } \\
\text { number }\end{array}$ & $\begin{array}{c}\text { Ammonia, } \\
\text { as nitrogen } \\
(\mathrm{mg} / \mathrm{L}) \\
(00608)\end{array}$ & $\begin{array}{l}\text { Nitrite, } \\
\text { as nitrogen } \\
(\mathbf{m g} / \mathrm{L}) \\
(00613)\end{array}$ & $\begin{array}{l}\text { Nitrite plus nitrate, } \\
\text { as nitrogen } \\
(\mathrm{mg} / \mathrm{L}) \\
(00631)\end{array}$ & $\begin{array}{c}\text { Total nitrogen }{ }^{1} \\
\text { (ammonia + nitrate + } \\
\text { nitrite + organic nitrogen) } \\
(\mathrm{mg} / \mathrm{L}) \\
(\mathbf{6 2 8 5 4 )}\end{array}$ & $\begin{array}{c}\text { Phosphate, } \\
\text { orthophosphate } \\
\text { (as phosphorus } \\
\text { (mg/L) } \\
\text { (00671) }\end{array}$ \\
\hline Benchmark type & HAL-US & MCL-US & MCL-US & na & na \\
\hline Benchmark level & ${ }^{2} 24.7$ & 1 & 10 & na & na \\
\hline [SRL], [IRL], or [LT-MDL] & ${ }^{3}[0.015]$ & ${ }^{3}[0.0014]$ & ${ }^{4}[0.04]$ & [0.05] & {$[0.004]$} \\
\hline \multicolumn{6}{|c|}{ Peninsular Ranges study area (50 wells sampled)—Continued } \\
\hline HR-PRFP-06 & - & - & - & - & 0.030 \\
\hline HR-PRFP-07 & - & - & - & - & 0.007 \\
\hline HR-PRFP-08 & - & - & - & - & 0.008 \\
\hline HR-PRFP-09 & - & 0.002 & 1.5 & 1.8 & 0.039 \\
\hline HR-PRFP-10 & - & 0.003 & 0.15 & 0.18 & 0.014 \\
\hline HR-PRFP-11 & - & $\leq 0.001$ & * 33.9 & 29.8 & 0.035 \\
\hline HR-PRFP-12 & - & - & $* 11.0$ & 10.5 & 0.046 \\
\hline HR-PRFP-13 & 0.03 & 0.118 & * 20.4 & 20.3 & 0.018 \\
\hline HR-PRU-01 & - & - & - & - & 0.007 \\
\hline
\end{tabular}

${ }^{1}$ Total nitrogen concentration ( $\mathrm{T}$ ) was compared with the sum of ammonia, nitrite, and nitrate concentrations (S) for each sample, using the same criteria used to evaluate acceptibility of replicates: for T greater than $0.25 \mathrm{mg} / \mathrm{L}$ ( 5 times the LT-MDL), relative percent difference less than 10 percent indicates acceptable replication; for T less than $0.25 \mathrm{mg} / \mathrm{L}$, standard deviation less than $0.025 \mathrm{mg} / \mathrm{L}$ indicates acceptable precision. There were five samples (HR-SC-09, HR-SC-10, HR-SC-12, HR-SC-16, and HR-SC-17) with T less than S, and precision between the two parameters was considered unacceptable. For T greater than S and unacceptable replication by these criteria, organic nitrogen may be present.

${ }^{2}$ The HAL-US is $30 \mathrm{mg} / \mathrm{L}$ "as ammonia." To facilitate comparson to the analytical results, we have converted and reported this HAL-US as $24.7 \mathrm{mg} / \mathrm{L}$ "as nitrogen."

${ }^{3}$ SRLs were defined for nutrients based on the highest detected concentrations in field blanks for the Hard Rock study unit. Values less than the SRL are reported as greater than or equal to $(\leq)$ the value reported by the laboratory. In the USGS National Water Information System (NWIS) database, the result is accompanied by the following comment: Result is < or = reported value, based on quality-control data. See appendix table A3 for more information.

\footnotetext{
${ }^{4}$ The interim reporting level is the highest reporting level for nitrite plus nitrate during March 2011 to March 2012 and therefore is used in this table. The SRL applied to nitrite plus nitrate, based on the highest concentration detected in field blanks for the Hard Rock study unit, is $0.031 \mathrm{mg} / \mathrm{L}$.

${ }^{5}$ Sample HR-SC-19 was diluted during analysis, increasing the LT-MDL of nitrite by a factor of two $(0.002 \mathrm{mg} / \mathrm{L})$.

${ }^{6}$ Nutrients were not detected in sample HR-SG-32. However, the sample was diluted, increasing the LT-MDLs for ammonia and orthophosphate by a factor of two $(0.020 \mathrm{mg} / \mathrm{L}$ and $0.008 \mathrm{mg} / \mathrm{L}$, respectively).
} 
Table 10. Major and minor ions, silica, and total dissolved solids (TDS) detected in samples collected for the Santa Cruz, San Gabriel, and Peninsular Ranges Hard Rock Aquifers (Hard Rock) study unit, March 2011 through March 2012, California GAMA Priority Basin Project.

[The five-digit U.S. Geological Survey (USGS) parameter code below the constituent name is used to uniquely identify a specific constituent or property. GAMA well identification number: HR-SC, Santa Cruz study area grid well; HR-SG, San Gabriel study area grid well; HR-SGU, San Gabriel study area understanding well; HR-PR, Peninsular Ranges study area grid well; HR-PRFP, Peninsular Ranges study area flow-path well; HR-PRU, Peninsular Ranges study area understanding well. Reporting levels, benchmark types, and benchmark levels as of July 6, 2012. Benchmark type: Maximum contaminant level benchmarks are listed as MCL-US when the MCL-US and MCL-CA are identical, and as MCL-CA when the MCL-CA is lower than the MCL-US or no MCL-US exists. Secondary maximum contaminant level benchmarks are listed as SMCL-CA when the SMCL-CA exists, and as SMCL-US when the SMCL-CA does not exist. MCL-CA, California Department of Public Health (CDPH) maximum contaminant level; SMCL-CA, CDPH secondary maximum contaminant level. Other abbreviations: LT-MDL, long-term method detection level; MRL, minimum reporting level; mg/L, milligrams per liter; SiO, silicon dioxide; na, not available; —, not detected; *, value above benchmark level, **, value above upper benchmark level]

\begin{tabular}{|c|c|c|c|c|c|c|c|c|c|c|c|}
\hline $\begin{array}{c}\text { GAMA } \\
\text { well } \\
\text { identification } \\
\text { number }\end{array}$ & $\begin{array}{c}\text { Bromide } \\
(\mathrm{mg} / \mathrm{L}) \\
(71870)\end{array}$ & $\begin{array}{l}\text { Calcium } \\
\text { (mg/L) } \\
(00915)\end{array}$ & $\begin{array}{l}\text { Chloride } \\
\text { (mg/L) } \\
(00940)\end{array}$ & $\begin{array}{l}\text { Fluoride } \\
\text { (mg/L) } \\
(00950)\end{array}$ & $\begin{array}{l}\text { lodide } \\
\text { (mg/L) } \\
(71865)\end{array}$ & $\begin{array}{l}\text { Magnesium } \\
(\mathrm{mg} / \mathrm{L}) \\
(00925)\end{array}$ & $\begin{array}{l}\text { Potassium } \\
\text { (mg/L) } \\
(00935)\end{array}$ & $\begin{array}{c}\text { Silica } \\
\text { (as SiO }) \\
(\mathrm{mg} / \mathrm{L}) \\
(00955)\end{array}$ & $\begin{array}{l}\text { Sodium } \\
\text { (mg/L) } \\
(00930)\end{array}$ & $\begin{array}{l}\text { Sulfate } \\
\text { (mg/L) } \\
(00945)\end{array}$ & $\begin{array}{c}\text { TDS } \\
\text { (as residue on evaporation) } \\
\text { (mg/L) } \\
(70300)\end{array}$ \\
\hline Benchmark type & na & na & SMCL-CA & MCL-CA & na & na & na & na & na & SMCL-CA & SMCL-CA \\
\hline Benchmark level & na & na & ${ }^{1} 250(500)$ & 2 & na & na & na & na & na & $1250(500)$ & ${ }^{1} 500(1,000)$ \\
\hline [LT-MDL] or [MRL] & {$[0.010]$} & {$[0.022]$} & {$[0.06]$} & {$[0.04]$} & {$[0.001]$} & {$[0.011]$} & {$[0.03]$} & {$[0.029]$} & {$[0.06]$} & {$[0.09]$} & [20] \\
\hline \multicolumn{12}{|c|}{ Santa Cruz study area (25 wells sampled) } \\
\hline HR-SC-01 & 0.138 & 28.7 & 40.2 & 0.57 & - & 3.48 & 0.36 & 16.8 & 23.9 & 10.3 & 166 \\
\hline HR-SC-02 & 0.138 & 26.3 & 33.2 & 0.18 & -2 & 11.2 & 1.61 & 33.8 & 45.5 & 58.0 & 318 \\
\hline HR-SC-03 & 0.549 & 20.4 & 76.9 & 1.74 & 0.195 & 15.9 & 2.56 & 16.3 & 107 & 32.0 & 415 \\
\hline HR-SC-04 & 0.054 & 146 & 31.7 & 0.14 & - & 20.3 & 2.06 & 18.7 & 22.9 & 203 & *609 \\
\hline HR-SC-05 & 0.043 & 33.4 & 8.40 & 0.11 & 0.003 & 8.72 & 2.46 & 30.7 & 9.75 & 16.7 & 170 \\
\hline HR-SC-06 & 0.046 & 60.4 & 13.6 & 0.18 & 0.001 & 10.1 & 1.36 & 28.4 & 16.3 & 26.2 & 257 \\
\hline HR-SC-07 & 0.904 & 86.4 & $* 258$ & 0.21 & 0.094 & 68.8 & 5.15 & 29.4 & 174 & 175 & $* * 1,020$ \\
\hline HR-SC-08 & 0.039 & 56.6 & 13.5 & 0.16 & 0.003 & 10.4 & 1.70 & 32.2 & 18.9 & 36.9 & 286 \\
\hline HR-SC-09 & 0.039 & 3.01 & 13.8 & $* 2.42$ & 0.039 & 0.298 & 1.63 & 11.5 & 240 & 13.3 & *620 \\
\hline HR-SC-10 & 0.028 & 50.5 & 4.32 & 0.17 & - & 3.25 & 0.80 & 35.8 & 9.78 & 17.2 & 212 \\
\hline HR-SC-11 & 0.080 & 128 & 34.0 & 0.11 & - & 15.9 & 0.71 & 16.9 & 14.3 & 13.3 & 425 \\
\hline HR-SC-12 & 0.059 & 87.2 & 17.6 & 0.24 & 0.002 & 64.8 & 0.90 & 40.6 & 22.9 & 35.4 & *542 \\
\hline HR-SC-13 & 0.010 & 31.4 & 3.74 & 0.19 & - & 10.2 & 0.52 & 18.2 & 10.8 & 17.8 & 166 \\
\hline HR-SC-14 & 0.063 & 65.6 & 20.8 & 0.35 & 0.007 & 19.7 & 1.84 & 46.7 & 21.4 & 91.2 & 402 \\
\hline HR-SC-15 & 0.043 & 4.07 & 10.6 & 0.72 & 0.004 & 1.99 & 3.34 & 14.3 & 112 & 33.8 & 311 \\
\hline
\end{tabular}


Table 10. Major and minor ions, silica, and total dissolved solids (TDS) detected in samples collected for the Santa Cruz, San Gabriel, and Peninsular Ranges Hard Rock Aquifers (Hard Rock) study unit, March 2011 through March 2012, California GAMA Priority Basin Project.—Continued

[The five-digit U.S. Geological Survey (USGS) parameter code below the constituent name is used to uniquely identify a specific constituent or property. GAMA well identification number: HR-SC, Santa Cruz study area grid well; HR-SG, San Gabriel study area grid well; HR-SGU, San Gabriel study area understanding well; HR-PR, Peninsular Ranges study area grid well; HR-PRFP, Peninsular Ranges study area flow-path well; HR-PRU, Peninsular Ranges study area understanding well. Reporting levels, benchmark types, and benchmark levels as of July 6, 2012. Benchmark type: Maximum contaminant level benchmarks are listed as MCL-US when the MCL-US and MCL-CA are identical, and as MCL-CA when the MCL-CA is lower than the MCL-US or no MCL-US exists. Secondary maximum contaminant level benchmarks are listed as SMCL-CA when the SMCL-CA exists, and as SMCL-US when the SMCL-CA does not exist. MCL-CA, California Department of Public Health (CDPH) maximum contaminant level; SMCL-CA, CDPH secondary maximum contaminant level. Other abbreviations: LT-MDL, long-term method detection level; MRL, minimum reporting level; mg/L, milligrams per liter; SiO ${ }_{2}$, silicon dioxide; na, not available; - , not detected; *, value above benchmark level, **, value above upper benchmark level]

\begin{tabular}{|c|c|c|c|c|c|c|c|c|c|c|c|}
\hline $\begin{array}{c}\text { GAMA } \\
\text { well } \\
\text { identification } \\
\text { number }\end{array}$ & $\begin{array}{l}\text { Bromide } \\
\text { (mg/L) } \\
(71870)\end{array}$ & $\begin{array}{l}\text { Calcium } \\
\text { (mg/L) } \\
(00915)\end{array}$ & $\begin{array}{l}\text { Chloride } \\
\text { (mg/L) } \\
(00940)\end{array}$ & $\begin{array}{l}\text { Fluoride } \\
\text { (mg/L) } \\
(00950)\end{array}$ & $\begin{array}{l}\text { lodide } \\
\text { (mg/L) } \\
(71865)\end{array}$ & $\begin{array}{l}\text { Magnesium } \\
(\mathrm{mg} / \mathrm{L}) \\
(00925)\end{array}$ & $\begin{array}{l}\text { Potassium } \\
\text { (mg/L) } \\
(00935)\end{array}$ & $\begin{array}{c}\text { Silica } \\
\left.\text { (as } \mathrm{SiO}_{2}\right) \\
(\mathrm{mg} / \mathrm{L}) \\
(00955)\end{array}$ & $\begin{array}{l}\text { Sodium } \\
\text { (mg/L) } \\
(00930)\end{array}$ & $\begin{array}{l}\text { Sulfate } \\
\text { (mg/L) } \\
(00945)\end{array}$ & $\begin{array}{c}\text { TDS } \\
\text { (as residue on evaporation) } \\
(\mathrm{mg} / \mathrm{L}) \\
(70300)\end{array}$ \\
\hline Benchmark type & na & na & SMCL-CA & MCL-CA & na & na & na & na & na & SMCL-CA & SMCL-CA \\
\hline Benchmark level & na & na & ${ }^{1} 250(500)$ & 2 & na & na & na & na & na & ${ }^{1} 250(500)$ & $1500(1,000)$ \\
\hline [LT-MDL] or [MRL] & {$[0.010]$} & {$[0.022]$} & {$[0.06]$} & {$[0.04]$} & {$[0.001]$} & {$[0.011]$} & {$[0.03]$} & [0.029] & {$[0.06]$} & {$[0.09]$} & [20] \\
\hline \multicolumn{12}{|c|}{ Santa Cruz study area ( 25 wells sampled) —Continued } \\
\hline HR-SC-16 & 0.015 & 29.8 & 6.35 & 0.12 & 0.002 & 10.5 & 2.33 & 15.7 & 15.9 & 14.9 & 158 \\
\hline HR-SC-17 & 0.020 & 6.22 & 7.27 & 0.06 & 0.001 & 1.21 & 0.95 & 45.2 & 8.72 & 7.51 & 95 \\
\hline HR-SC-18 & 0.025 & 22.0 & 8.36 & 0.10 & 0.005 & 1.47 & 1.28 & 37.8 & 16.6 & 27.7 & 154 \\
\hline HR-SC-19 & 0.200 & 37.5 & 41.7 & 0.32 & 0.059 & 37.6 & 4.67 & 36.3 & 71.1 & 35.6 & 436 \\
\hline HR-SC-20 & 0.491 & 50.6 & 236 & 0.24 & 0.004 & 64.5 & 1.17 & 26.2 & 192 & 152 & *912 \\
\hline HR-SC-21 & 0.138 & 45.6 & 64.0 & 0.19 & 0.003 & 37.2 & 11.6 & 56.9 & 150 & $* 268$ & *792 \\
\hline HR-SC-22 & 0.092 & 118 & 30.1 & 0.10 & 0.009 & 36.6 & 1.46 & 30.8 & 20.4 & 70.3 & $* 544$ \\
\hline HR-SC-23 & 0.060 & 80.0 & 19.6 & 0.31 & 0.003 & 28.4 & 3.81 & 29.6 & 60.3 & 59.8 & 480 \\
\hline HR-SC-24 & 0.206 & 133 & 119 & 0.29 & 0.028 & 89.0 & 2.43 & 25.1 & 81.8 & 142 & *941 \\
\hline HR-SC-25 & 0.016 & 16.5 & 7.73 & 0.18 & - & 1.02 & 0.97 & 33.2 & 10.6 & 8.19 & 102 \\
\hline \multicolumn{12}{|c|}{ San Gabriel study area (37 wells sampled) } \\
\hline HR-SG-01 & 0.054 & 71.1 & 20.2 & 0.90 & 0.003 & 36.1 & 1.80 & 21.1 & 36.9 & 134 & 482 \\
\hline HR-SG-02 & 0.055 & 73.4 & 20.3 & 0.84 & 0.002 & 28.8 & 2.52 & 23.6 & 29.9 & 67.3 & 413 \\
\hline HR-SG-03 & 0.103 & 70.3 & 24.4 & 0.32 & 0.004 & 18.0 & 1.96 & 28.1 & 40.5 & 47.4 & 408 \\
\hline HR-SG-04 & 0.278 & 118 & 79.1 & 0.39 & 0.001 & 48.2 & 1.62 & 28.1 & 45.4 & 141 & *734 \\
\hline HR-SG-05 & 0.041 & 80.0 & 13.1 & 0.58 & 0.001 & 23.8 & 2.40 & 18.1 & 31.8 & 52.2 & 404 \\
\hline HR-SG-06 & 0.029 & 36.4 & 6.39 & 0.19 & - & 12.3 & 4.27 & 20.2 & 17.2 & 29.4 & 207 \\
\hline HR-SG-07 & 0.022 & 45.5 & 4.50 & 0.88 & 0.005 & 8.92 & 0.60 & 22.6 & 17.3 & 18.7 & 216 \\
\hline HR-SG-08 & 0.177 & 90.5 & 46.3 & 0.33 & 0.019 & 23.7 & 2.48 & 30.0 & 62.0 & 56.7 & $* 515$ \\
\hline HR-SG-09 & 0.209 & 4.10 & 62.0 & 1.47 & 0.038 & 0.199 & 0.88 & 37.7 & 274 & 232 & *780 \\
\hline HR-SG-10 & 0.054 & 67.0 & 13.5 & 1.51 & 0.001 & 25.6 & 2.82 & 24.1 & 31.8 & 64.8 & 380 \\
\hline
\end{tabular}


Table 10. Major and minor ions, silica, and total dissolved solids (TDS) detected in samples collected for the Santa Cruz, San Gabriel, and Peninsular Ranges Hard Rock Aquifers (Hard Rock) study unit, March 2011 through March 2012, California GAMA Priority Basin Project.-Continued

[The five-digit U.S. Geological Survey (USGS) parameter code below the constituent name is used to uniquely identify a specific constituent or property. GAMA well identification number: HR-SC, Santa Cruz study area grid well; HR-SG, San Gabriel study area grid well; HR-SGU, San Gabriel study area understanding well; HR-PR, Peninsular Ranges study area grid well; HR-PRFP, Peninsular Ranges study area flow-path well; HR-PRU, Peninsular Ranges study area understanding well. Reporting levels, benchmark types, and benchmark levels as of July 6, 2012. Benchmark type: Maximum contaminant level benchmarks are listed as MCL-US when the MCL-US and MCL-CA are identical, and as MCL-CA when the MCL-CA is lower than the MCL-US or no MCL-US exists. Secondary maximum contaminant level benchmarks are listed as SMCL-CA when the SMCL-CA exists, and as SMCL-US when the SMCL-CA does not exist. MCL-CA, California Department of Public Health (CDPH) maximum contaminant level; SMCL-CA, CDPH secondary maximum contaminant level. Other abbreviations: LT-MDL, long-term method detection level; MRL, minimum reporting level; mg/L, milligrams per liter; SiO, silicon dioxide; na, not available; - , not detected; *, value above benchmark level, **, value above upper benchmark level]

\begin{tabular}{|c|c|c|c|c|c|c|c|c|c|c|c|}
\hline $\begin{array}{c}\text { GAMA } \\
\text { well } \\
\text { identification } \\
\text { number }\end{array}$ & $\begin{array}{c}\text { Bromide } \\
(\mathrm{mg} / \mathrm{L}) \\
(71870)\end{array}$ & $\begin{array}{l}\text { Calcium } \\
\text { (mg/L) } \\
(00915)\end{array}$ & $\begin{array}{l}\text { Chloride } \\
\text { (mg/L) } \\
(00940)\end{array}$ & $\begin{array}{l}\text { Fluoride } \\
\text { (mg/L) } \\
(00950)\end{array}$ & $\begin{array}{l}\text { lodide } \\
\text { (mg/L) } \\
(71865)\end{array}$ & $\begin{array}{l}\text { Magnesium } \\
(\mathrm{mg} / \mathrm{L}) \\
(00925)\end{array}$ & $\begin{array}{l}\text { Potassium } \\
\text { (mg/L) } \\
(00935)\end{array}$ & $\begin{array}{c}\text { Silica } \\
\left.\text { (as } \mathrm{SiO}_{2}\right) \\
(\mathrm{mg} / \mathrm{L}) \\
(00955)\end{array}$ & $\begin{array}{l}\text { Sodium } \\
\text { (mg/L) } \\
(00930)\end{array}$ & $\begin{array}{l}\text { Sulfate } \\
\text { (mg/L) } \\
(00945)\end{array}$ & $\begin{array}{c}\text { TDS } \\
\text { (as residue on evaporation) } \\
(\mathrm{mg} / \mathrm{L}) \\
(70300)\end{array}$ \\
\hline Benchmark type & na & na & SMCL-CA & MCL-CA & na & na & na & na & na & SMCL-CA & SMCL-CA \\
\hline Benchmark level & na & na & ${ }^{1} 250(500)$ & 2 & na & na & na & na & na & ${ }^{1} 250(500)$ & ${ }^{1} 500(1,000)$ \\
\hline [LT-MDL] or [MRL] & {$[0.010]$} & {$[0.022]$} & {$[0.06]$} & {$[0.04]$} & {$[0.001]$} & {$[0.011]$} & {$[0.03]$} & {$[0.029]$} & {$[0.06]$} & {$[0.09]$} & [20] \\
\hline \multicolumn{12}{|c|}{ San Gabriel study area (37 wells sampled)—Continued } \\
\hline HR-SG-11 & 0.058 & 18.4 & 13.1 & $* 8.67$ & 0.006 & 8.28 & 0.84 & 24.4 & 46.4 & 17.7 & 193 \\
\hline HR-SG-12 & - & 49.0 & 2.64 & 0.21 & - & 12.0 & 2.28 & 20.9 & 6.55 & 2.35 & 199 \\
\hline HR-SG-13 & 0.014 & 71.4 & 3.00 & 0.25 & - & 20.5 & 2.18 & 14.7 & 17.9 & 26.7 & 326 \\
\hline HR-SG-14 & 0.013 & 102 & 2.51 & 0.09 & - & 20.3 & 2.80 & 18.9 & 4.15 & 16.0 & 373 \\
\hline HR-SG-15 & - & 44.3 & 0.83 & 0.57 & - & 6.79 & 3.30 & 9.38 & 3.65 & 7.66 & 167 \\
\hline HR-SG-16 & 0.038 & 58.6 & 7.46 & 0.90 & - & 12.0 & 0.22 & 19.9 & 13.8 & 9.70 & 249 \\
\hline HR-SG-17 & - & 52.9 & 1.49 & 0.13 & - & 3.86 & 1.53 & 25.7 & 9.34 & 2.38 & 212 \\
\hline HR-SG-18 & 0.072 & 62.1 & 11.4 & 0.21 & 0.002 & 16.0 & 2.25 & 31.1 & 34.6 & 34.0 & 312 \\
\hline HR-SG-19 & 0.089 & 75.3 & 13.5 & 0.38 & 0.003 & 13.6 & 3.92 & 18.9 & 25.8 & 33.0 & 341 \\
\hline HR-SG-20 & - & 69.6 & 3.04 & 0.45 & - & 35.3 & 4.27 & 12.7 & 29.7 & 85.1 & 426 \\
\hline HR-SG-21 & - & 66.6 & 4.08 & - & 0.001 & 13.0 & 0.65 & 24.3 & 12.8 & 14.9 & 295 \\
\hline HR-SG-22 & 0.065 & 49.1 & 30.4 & 0.76 & 0.038 & 16.2 & 3.36 & 21.6 & 44.8 & 34.5 & 323 \\
\hline HR-SG-23 & - & 38.2 & 1.97 & 0.27 & 0.001 & 6.25 & 2.14 & 18.3 & 6.86 & 9.11 & 153 \\
\hline HR-SG-24 & 0.039 & 38.8 & 9.59 & $* 3.49$ & 0.005 & 16.2 & 1.76 & 24.8 & 33.8 & 58.1 & 295 \\
\hline HR-SG-25 & 0.040 & 16.8 & 6.92 & 0.34 & - & 11.1 & 2.59 & 27.8 & 18.4 & 8.80 & 149 \\
\hline HR-SG-26 & 0.017 & 52.3 & 2.74 & 0.39 & - & 12.7 & 3.14 & 12.7 & 8.53 & 24.0 & 227 \\
\hline HR-SG-27 & 0.017 & 31.3 & 3.24 & 0.16 & - & 5.36 & 2.24 & 29.5 & 11.2 & 3.85 & 154 \\
\hline HR-SG-28 & - & 40.8 & 2.37 & 0.10 & - & 7.70 & 2.19 & 21.0 & 8.28 & 16.9 & 174 \\
\hline HR-SG-29 & - & 83.2 & 0.80 & 0.09 & - & 6.12 & 1.79 & 11.8 & 4.16 & 38.4 & 274 \\
\hline HR-SG-30 & - & 69.5 & 0.89 & 0.14 & - & 7.30 & 1.12 & 9.69 & 3.67 & 13.9 & 236 \\
\hline
\end{tabular}


Table 10. Major and minor ions, silica, and total dissolved solids (TDS) detected in samples collected for the Santa Cruz, San Gabriel, and Peninsular Ranges Hard Rock Aquifers (Hard Rock) study unit, March 2011 through March 2012, California GAMA Priority Basin Project.-Continued

[The five-digit U.S. Geological Survey (USGS) parameter code below the constituent name is used to uniquely identify a specific constituent or property. GAMA well identification number: HR-SC, Santa Cruz study area grid well; HR-SG, San Gabriel study area grid well; HR-SGU, San Gabriel study area understanding well; HR-PR, Peninsular Ranges study area grid well; HR-PRFP, Peninsular Ranges study area flow-path well; HR-PRU, Peninsular Ranges study area understanding well. Reporting levels, benchmark types, and benchmark levels as of July 6, 2012. Benchmark type: Maximum contaminant level benchmarks are listed as MCL-US when the MCL-US and MCL-CA are identical, and as MCL-CA when the MCL-CA is lower than the MCL-US or no MCL-US exists. Secondary maximum contaminant leve benchmarks are listed as SMCL-CA when the SMCL-CA exists, and as SMCL-US when the SMCL-CA does not exist. MCL-CA, California Department of Public Health (CDPH) maximum contaminant level; SMCL-CA, CDPH secondary maximum contaminant level. Other abbreviations: LT-MDL, long-term method detection level; MRL, minimum reporting level; mg/L, milligrams per liter; SiO ${ }_{2}$, silicon dioxide; na, not available; - , not detected; *, value above benchmark level, **, value above upper benchmark level]

\begin{tabular}{|c|c|c|c|c|c|c|c|c|c|c|c|}
\hline $\begin{array}{c}\text { GAMA } \\
\text { well } \\
\text { identification } \\
\text { number }\end{array}$ & $\begin{array}{c}\text { Bromide } \\
\text { (mg/L) } \\
(71870)\end{array}$ & $\begin{array}{l}\text { Calcium } \\
(\mathrm{mg} / \mathrm{L}) \\
(00915)\end{array}$ & $\begin{array}{c}\text { Chloride } \\
\text { (mg/L) } \\
(00940)\end{array}$ & $\begin{array}{c}\text { Fluoride } \\
(\mathrm{mg} / \mathrm{L}) \\
(00950)\end{array}$ & $\begin{array}{l}\text { lodide } \\
\text { (mg/L) } \\
(71865)\end{array}$ & $\begin{array}{c}\text { Magnesium } \\
(\mathrm{mg} / \mathrm{L}) \\
(00925)\end{array}$ & $\begin{array}{l}\text { Potassium } \\
\text { (mg/L) } \\
(00935)\end{array}$ & $\begin{array}{c}\text { Silica } \\
\left(\mathrm{as} \mathrm{SiO}_{2}\right) \\
(\mathrm{mg} / \mathrm{L}) \\
(00955)\end{array}$ & $\begin{array}{l}\text { Sodium } \\
\text { (mg/L) } \\
(00930)\end{array}$ & $\begin{array}{l}\text { Sulfate } \\
\text { (mg/L) } \\
(00945)\end{array}$ & $\begin{array}{c}\text { TDS } \\
\text { (as residue on evaporation) } \\
(\mathrm{mg} / \mathrm{L}) \\
(70300)\end{array}$ \\
\hline Benchmark type & na & na & SMCL-CA & MCL-CA & na & na & na & na & na & SMCL-CA & SMCL-CA \\
\hline Benchmark level & na & na & $1250(500)$ & 2 & na & na & na & na & na & ${ }^{1} 250(500)$ & ${ }^{1} 500(1,000)$ \\
\hline [LT-MDL] or [MRL] & {$[0.010]$} & {$[0.022]$} & {$[0.06]$} & {$[0.04]$} & {$[0.001]$} & {$[0.011]$} & [0.03] & {$[0.029]$} & {$[0.06]$} & [0.09] & [20] \\
\hline \multicolumn{12}{|c|}{ San Gabriel study area (37 wells sampled)—Continued } \\
\hline HR-SG-31 & 0.066 & 62.5 & 17.1 & 1.02 & 0.004 & 34.4 & 1.81 & 29.9 & 30.3 & 61.1 & 404 \\
\hline HR-SG-32 & 0.042 & 18.6 & 4.98 & 0.06 & 0.004 & 5.02 & 0.88 & 24.0 & 12.6 & 3.01 & 113 \\
\hline HR-SG-33 & 0.471 & 87.4 & 87.0 & 0.54 & 0.002 & 27.3 & 1.81 & 32.9 & 53.2 & 82.8 & $* 521$ \\
\hline HR-SGU-01 & 0.040 & 39.1 & 61.0 & 1.06 & 0.002 & 46.5 & 4.12 & 22.2 & 76.8 & 35.7 & $* 506$ \\
\hline HR-SGU-02 & - & 27.2 & 1.53 & 0.09 & - & 3.44 & 1.84 & 15.1 & 6.80 & 3.55 & 124 \\
\hline HR-SGU-03 & - & 34.6 & 0.78 & 0.36 & - & 7.58 & 2.18 & 14.5 & 3.13 & 24.6 & 150 \\
\hline HR-SGU-04 & - & 74.0 & 0.61 & - & - & 4.16 & 1.63 & 9.17 & 3.13 & 31.5 & 240 \\
\hline \multicolumn{12}{|c|}{ Peninsular Ranges study area ( 50 wells sampled) } \\
\hline HR-PR-01 & 0.329 & 55.8 & 91.0 & 0.27 & 0.038 & 38.5 & 2.82 & 48.5 & 57.7 & 21.9 & $* 516$ \\
\hline HR-PR-02 & 0.312 & 38.8 & 58.0 & 0.31 & 0.004 & 11.0 & 5.23 & 38.9 & 53.2 & 6.44 & 338 \\
\hline HR-PR-03 & 0.020 & 15.0 & 6.31 & 0.05 & 0.005 & 7.82 & 3.20 & 35.5 & 14.6 & 9.50 & 143 \\
\hline HR-PR-04 & 0.069 & 25.2 & 19.8 & 0.15 & 0.007 & 10.5 & 6.43 & 55.6 & 24.5 & 19.7 & 231 \\
\hline HR-PR-05 & 0.043 & 18.5 & 10.7 & 0.23 & 0.005 & 6.48 & 2.79 & 27.7 & 18.7 & 12.6 & 158 \\
\hline HR-PR-06 & 0.296 & 79.3 & 114 & 0.37 & 0.037 & 7.05 & 1.30 & 21.8 & 97.6 & 64.6 & *537 \\
\hline HR-PR-07 & 0.607 & 151 & *328 & 0.44 & 0.067 & 100 & 5.06 & 50.8 & 210 & $* 481$ & $* * 1,590$ \\
\hline HR-PR-08 & 0.080 & 41.5 & 21.7 & 0.11 & 0.001 & 8.92 & 0.57 & 30.2 & 27.6 & 34.7 & 260 \\
\hline HR-PR-09 & 0.069 & 35.9 & 24.2 & 0.11 & 0.003 & 8.69 & 1.33 & 25.8 & 22.1 & 5.26 & 216 \\
\hline HR-PR-10 & 0.143 & 64.1 & 46.8 & 0.06 & 0.009 & 29.0 & 3.51 & 46.2 & 36.7 & 212 & $* 515$ \\
\hline
\end{tabular}


Table 10. Major and minor ions, silica, and total dissolved solids (TDS) detected in samples collected for the Santa Cruz, San Gabriel, and Peninsular Ranges Hard Rock Aquifers (Hard Rock) study unit, March 2011 through March 2012, California GAMA Priority Basin Project.-Continued

[The five-digit U.S. Geological Survey (USGS) parameter code below the constituent name is used to uniquely identify a specific constituent or property. GAMA well identification number: HR-SC, Santa Cruz study area grid well; HR-SG, San Gabriel study area grid well; HR-SGU, San Gabriel study area understanding well; HR-PR, Peninsular Ranges study area grid well; HR-PRFP, Peninsular Ranges study area flow-path well; HR-PRU, Peninsular Ranges study area understanding well. Reporting levels, benchmark types, and benchmark levels as of July 6, 2012. Benchmark type: Maximum contaminant level benchmarks are listed as MCL-US when the MCL-US and MCL-CA are identical, and as MCL-CA when the MCL-CA is lower than the MCL-US or no MCL-US exists. Secondary maximum contaminant level benchmarks are listed as SMCL-CA when the SMCL-CA exists, and as SMCL-US when the SMCL-CA does not exist. MCL-CA, California Department of Public Health (CDPH) maximum contaminant level; SMCL-CA, CDPH secondary maximum contaminant level. Other abbreviations: LT-MDL, long-term method detection level; MRL, minimum reporting level; mg/L, milligrams per liter; SiO, silicon dioxide; na, not available; —, not detected; *, value above benchmark level, **, value above upper benchmark level]

\begin{tabular}{|c|c|c|c|c|c|c|c|c|c|c|c|}
\hline $\begin{array}{c}\text { GAMA } \\
\text { well } \\
\text { identification } \\
\text { number }\end{array}$ & $\begin{array}{c}\text { Bromide } \\
(\mathrm{mg} / \mathrm{L}) \\
(71870)\end{array}$ & $\begin{array}{l}\text { Calcium } \\
\text { (mg/L) } \\
(00915)\end{array}$ & $\begin{array}{l}\text { Chloride } \\
\text { (mg/L) } \\
(00940)\end{array}$ & $\begin{array}{c}\text { Fluoride } \\
\text { (mg/L) } \\
(00950)\end{array}$ & $\begin{array}{l}\text { lodide } \\
\text { (mg/L) } \\
(71865)\end{array}$ & $\begin{array}{l}\text { Magnesium } \\
(\mathrm{mg} / \mathrm{L}) \\
(00925)\end{array}$ & $\begin{array}{l}\text { Potassium } \\
\text { (mg/L) } \\
(00935)\end{array}$ & $\begin{array}{c}\text { Silica } \\
\text { (as SiO }) \\
\left(\mathrm{mg}_{2}\right) \\
(00955)\end{array}$ & $\begin{array}{l}\text { Sodium } \\
(\mathrm{mg} / \mathrm{L}) \\
(00930)\end{array}$ & $\begin{array}{l}\text { Sulfate } \\
\text { (mg/L) } \\
(00945)\end{array}$ & $\begin{array}{c}\text { TDS } \\
\text { (as residue on evaporation) } \\
(\mathrm{mg} / \mathrm{L}) \\
(70300)\end{array}$ \\
\hline Benchmark type & na & na & SMCL-CA & MCL-CA & na & na & na & na & na & SMCL-CA & SMCL-CA \\
\hline Benchmark level & na & na & ${ }^{1} 250(500)$ & 2 & na & na & na & na & na & $1250(500)$ & $1500(1,000)$ \\
\hline [LT-MDL] or [MRL] & {$[0.010]$} & {$[0.022]$} & {$[0.06]$} & {$[0.04]$} & {$[0.001]$} & {$[0.011]$} & {$[0.03]$} & {$[0.029]$} & {$[0.06]$} & {$[0.09]$} & [20] \\
\hline \multicolumn{12}{|c|}{ Peninsular Ranges study area (50 wells sampled)_-Continued } \\
\hline HR-PR-11 & 0.373 & 46.6 & 107 & 0.34 & 0.015 & 22.0 & 2.52 & 40.0 & 54.3 & 37.2 & 393 \\
\hline HR-PR-12 & 0.133 & 58.7 & 93.4 & 0.19 & 0.002 & 32.5 & 1.88 & 39.0 & 73.7 & 191 & $* 595$ \\
\hline HR-PR-13 & 0.536 & 98.1 & 222 & 0.32 & 0.014 & 43.8 & 4.23 & 37.0 & 129 & 66.9 & *839 \\
\hline HR-PR-14 & 0.333 & 47.6 & 70.9 & 0.29 & 0.005 & 14.8 & 4.28 & 39.7 & 55.2 & 31.1 & 379 \\
\hline HR-PR-15 & 0.015 & 15.2 & 13.4 & - & - & 7.56 & 3.82 & 30.2 & 12.8 & 5.25 & 137 \\
\hline HR-PR-16 & 0.817 & 65.7 & 241 & 0.33 & 0.128 & 32.0 & 4.65 & 37.8 & 135 & 72.3 & *709 \\
\hline HR-PR-17 & 0.210 & 47.5 & 43.0 & 0.32 & 0.002 & 16.4 & 3.07 & 34.3 & 47.4 & 17.7 & 356 \\
\hline HR-PR-18 & 0.528 & 94.9 & 67.8 & 0.44 & 0.601 & 21.4 & 3.35 & 42.4 & 69.2 & 35.8 & *575 \\
\hline HR-PR-19 & 0.143 & 34.0 & 27.9 & 0.34 & 0.004 & 17.5 & 3.34 & 46.0 & 32.3 & 21.9 & 277 \\
\hline HR-PR-20 & 0.099 & 59.1 & 23.6 & 0.26 & 0.002 & 14.7 & 1.46 & 43.0 & 28.9 & 81.8 & 347 \\
\hline HR-PR-21 & 0.511 & 91.3 & 194 & 0.64 & 0.037 & 35.7 & 4.24 & 41.5 & 89.7 & 83.8 & $* 672$ \\
\hline HR-PR-22 & 0.252 & 106 & 77.0 & 0.17 & 0.016 & 24.0 & 5.38 & 32.0 & 42.7 & 88.3 & $* 538$ \\
\hline HR-PR-23 & 0.159 & 40.3 & 51.0 & 0.36 & 0.029 & 17.0 & 6.75 & 68.2 & 41.3 & 24.6 & 355 \\
\hline HR-PR-24 & 0.375 & 106 & 197 & 0.28 & 0.006 & 37.1 & 6.04 & 79.1 & 78.9 & 149 & *777 \\
\hline HR-PR-25 & 0.237 & 89.7 & 89.9 & - & 0.004 & 63.5 & 2.74 & 65.0 & 25.3 & 71.4 & $* 590$ \\
\hline HR-PR-26 & 1.84 & 185 & $* * 639$ & 0.28 & 0.065 & 131 & 0.47 & 34.8 & 213 & 228 & $* * 1,590$ \\
\hline HR-PR-27 & 0.052 & 18.1 & 12.2 & 0.19 & - & 5.52 & 1.34 & 36.3 & 19.4 & 1.77 & 157 \\
\hline HR-PR-28 & 0.370 & 138 & 93.7 & 0.35 & 0.021 & 35.4 & 9.90 & 40.3 & 74.0 & $* 327$ & *850 \\
\hline HR-PR-29 & 0.247 & 3.94 & 39.3 & 0.36 & 0.019 & 0.037 & 0.73 & 13.8 & 56.9 & 23.7 & 179 \\
\hline HR-PR-30 & 0.061 & 28.9 & 17.9 & 0.18 & 0.005 & 5.07 & 3.62 & 39.8 & 28.3 & 7.25 & 204 \\
\hline
\end{tabular}


Table 10. Major and minor ions, silica, and total dissolved solids (TDS) detected in samples collected for the Santa Cruz, San Gabriel, and Peninsular Ranges Hard Rock Aquifers (Hard Rock) study unit, March 2011 through March 2012, California GAMA Priority Basin Project.—Continued

[The five-digit U.S. Geological Survey (USGS) parameter code below the constituent name is used to uniquely identify a specific constituent or property. GAMA well identification number: HR-SC, Santa Cruz study area grid well; HR-SG, San Gabriel study area grid well; HR-SGU, San Gabriel study area understanding well; HR-PR, Peninsular Ranges study area grid well; HR-PRFP, Peninsular Ranges study area flow-path well; HR-PRU, Peninsular Ranges study area understanding well. Reporting levels, benchmark types, and benchmark levels as of July 6, 2012. Benchmark type: Maximum contaminant level benchmarks are listed as MCL-US when the MCL-US and MCL-CA are identical, and as MCL-CA when the MCL-CA is lower than the MCL-US or no MCL-US exists. Secondary maximum contaminant leve benchmarks are listed as SMCL-CA when the SMCL-CA exists, and as SMCL-US when the SMCL-CA does not exist. MCL-CA, California Department of Public Health (CDPH) maximum contaminant level; SMCL-CA, CDPH secondary maximum contaminant level. Other abbreviations: LT-MDL, long-term method detection level; MRL, minimum reporting level; mg/L, milligrams per liter; SiO , silicon dioxide; na, not available; - , not detected; *, value above benchmark level, **, value above upper benchmark level]

\begin{tabular}{|c|c|c|c|c|c|c|c|c|c|c|c|}
\hline $\begin{array}{c}\text { GAMA } \\
\text { well } \\
\text { identification } \\
\text { number }\end{array}$ & $\begin{array}{c}\text { Bromide } \\
(\mathrm{mg} / \mathrm{L}) \\
(71870)\end{array}$ & $\begin{array}{l}\text { Calcium } \\
\text { (mg/L) } \\
(00915)\end{array}$ & $\begin{array}{l}\text { Chloride } \\
\text { (mg/L) } \\
(00940)\end{array}$ & $\begin{array}{l}\text { Fluoride } \\
\text { (mg/L) } \\
(00950)\end{array}$ & $\begin{array}{l}\text { lodide } \\
(\mathrm{mg} / \mathrm{L}) \\
(71865)\end{array}$ & $\begin{array}{l}\text { Magnesium } \\
(\mathrm{mg} / \mathrm{L}) \\
(00925)\end{array}$ & $\begin{array}{l}\text { Potassium } \\
\text { (mg/L) } \\
(00935)\end{array}$ & $\begin{array}{c}\text { Silica } \\
\left(\mathrm{as} \mathrm{SiO}_{2}\right) \\
(\mathrm{mg} / \mathrm{L}) \\
(00955)\end{array}$ & $\begin{array}{l}\text { Sodium } \\
\text { (mg/L) } \\
(00930)\end{array}$ & $\begin{array}{l}\text { Sulfate } \\
\text { (mg/L) } \\
(00945)\end{array}$ & $\begin{array}{c}\text { TDS } \\
\text { (as residue on evaporation) } \\
\text { (mg/L) } \\
(70300)\end{array}$ \\
\hline Benchmark type & na & na & SMCL-CA & MCL-CA & na & na & na & na & na & SMCL-CA & SMCL-CA \\
\hline Benchmark level & na & na & ${ }^{1} 250(500)$ & 2 & na & na & na & na & na & ${ }^{1} 250(500)$ & $1500(1,000)$ \\
\hline [LT-MDL] or [MRL] & {$[0.010]$} & {$[0.022]$} & {$[0.06]$} & {$[0.04]$} & {$[0.001]$} & {$[0.011]$} & {$[0.03]$} & {$[0.029]$} & {$[0.06]$} & {$[0.09]$} & [20] \\
\hline \multicolumn{12}{|c|}{ Peninsular Ranges study area (50 wells sampled)—Continued } \\
\hline HR-PR-31 & - & 13.7 & 9.80 & 0.07 & - & 1.41 & 1.27 & 23.8 & 20.0 & 2.98 & 105 \\
\hline HR-PR-32 & 0.312 & 129 & 55.1 & 0.21 & 0.018 & 61.5 & 0.56 & 37.5 & 53.8 & *308 & $* 820$ \\
\hline HR-PR-33 & 0.298 & 57.0 & 60.9 & 0.45 & 0.006 & 16.1 & 1.51 & 54.6 & 64.2 & 22.6 & 451 \\
\hline HR-PR-34 & 0.373 & 65.8 & 72.3 & 0.84 & 0.004 & 18.1 & 1.05 & 47.2 & 82.1 & 26.9 & 466 \\
\hline HR-PR-35 & 0.082 & 31.3 & 15.6 & 0.23 & 0.002 & 7.05 & 2.61 & 40.6 & 21.9 & 5.02 & 205 \\
\hline HR-PR-36 & 0.023 & 18.8 & 5.56 & 0.08 & 0.004 & 7.89 & 1.83 & 26.8 & 14.8 & 0.75 & 148 \\
\hline HR-PRFP-01 & 0.157 & 57.1 & 45.9 & 0.50 & 0.016 & 13.1 & 3.57 & 52.5 & 34.7 & 64.5 & 365 \\
\hline HR-PRFP-02 & 0.256 & 74.7 & 171 & 0.37 & 0.009 & 57.9 & 2.71 & 51.1 & 121 & 226 & $* 873$ \\
\hline HR-PRFP-03 & 0.294 & 79.0 & 88.6 & 0.45 & 0.015 & 20.5 & 5.35 & 49.7 & 51.9 & 97.2 & 496 \\
\hline HR-PRFP-04 & 0.360 & 60.6 & 131 & 0.25 & 0.003 & 17.9 & 2.63 & 39.5 & 79.4 & 34.3 & $* 506$ \\
\hline HR-PRFP-05 & 0.502 & 130 & 187 & 0.30 & 0.083 & 40.8 & 6.08 & 45.1 & 91.4 & 152 & *830 \\
\hline HR-PRFP-06 & 0.317 & 88.3 & 115 & 0.23 & 0.084 & 19.6 & 4.85 & 44.9 & 64.0 & 67.5 & *540 \\
\hline HR-PRFP-07 & 0.107 & 45.8 & 30.8 & 0.39 & 0.006 & 10.7 & 2.77 & 26.7 & 27.9 & 69.8 & 279 \\
\hline HR-PRFP-08 & 0.065 & 7.69 & 17.1 & $* 2.93$ & 0.008 & 0.518 & 0.70 & 24.3 & 34.4 & 14.7 & 127 \\
\hline HR-PRFP-09 & 0.603 & 121 & $* 288$ & 0.51 & 0.042 & 73.4 & 9.74 & 39.5 & 184 & $* 256$ & $* * 1,210$ \\
\hline HR-PRFP-10 & 0.126 & 43.6 & 48.3 & 0.28 & 0.015 & 12.9 & 4.07 & 42.9 & 31.6 & 36.4 & 309 \\
\hline HR-PRFP-11 & 3.43 & 448 & $* * 1,290$ & 0.17 & 0.034 & 133 & 3.39 & 30.7 & 325 & 223 & $* * 3,180$ \\
\hline HR-PRFP-12 & 0.718 & 128 & $* 259$ & 0.41 & 0.011 & 43.5 & 4.06 & 47.3 & 120 & 128 & *951 \\
\hline HR-PRFP-13 & 2.59 & 149 & $* * 753$ & 0.71 & 0.530 & 117 & 2.10 & 45.1 & 414 & $* 444$ & $* * 2,220$ \\
\hline HR-PRU-01 & 0.033 & 19.6 & 9.11 & - & 0.002 & 9.47 & 5.24 & 22.7 & 11.6 & 6.25 & 144 \\
\hline
\end{tabular}

${ }^{1}$ The SMCL-CAs for chloride, sulfate, and TDS have recommended and upper benchmark values. The upper value is shown in parentheses.

${ }^{2}$ The LT-MDL for iodide was raised by a factor of two $(0.002 \mathrm{mg} / \mathrm{L})$ for HR-SC-02 due to sample dilution during analysis. 
Table 11. Arsenic, chromium, and iron species detected in samples collected for the Santa Cruz, San Gabriel, and Peninsular Ranges Hard Rock Aquifers (Hard Rock) study unit, March 2011 through March 2012, California GAMA Priority Basin Project.

[The five-digit U.S. Geological Survey (USGS) parameter code below the constituent name is used to uniquely identify a specific constituent or property. Samples from 110 wells were analyzed for arsenic and iron species. Samples from all 37 San Gabriel study area wells were analyzed for chromium species. Only samples with detections of at least one constituent are listed. GAMA well identification number: HR-SC, Santa Cruz study area grid well; HR-SG, San Gabriel study area grid well; HR-SGU, San Gabriel study area understanding well; HR-PR, Peninsular Ranges study area grid well; HR-PRFP, Peninsular Ranges study area flow-path well; HR-PRU, Peninsular Ranges study area understanding well. Reporting levels, benchmark types, and benchmark levels as of July 6, 2012. Benchmark type: Maximum contaminant level benchmarks are listed as MCL-US when the MCL-US and MCL-CA are identical, and as MCL-CA when the MCL-CA is lower than the MCL-US or no MCL-US exists. Secondary maximum contaminant level benchmarks are listed as SMCL-CA when the SMCL-CA exists, and as the SMCL-US when the SMCL-CA does not exist. MCL-US, U.S. Environmental Protection Agency maximum contaminant level; MCL-CA, California Department of Public Health maximum contaminant level; SMCL-CA, California Department of Public Health secondary maximum contaminant level. Other abbreviations: MDL, method detection limit; $\mu \mathrm{g} / \mathrm{L}$, micrograms per liter; 一, not detected; na, not available; *, greater than the benchmark level]

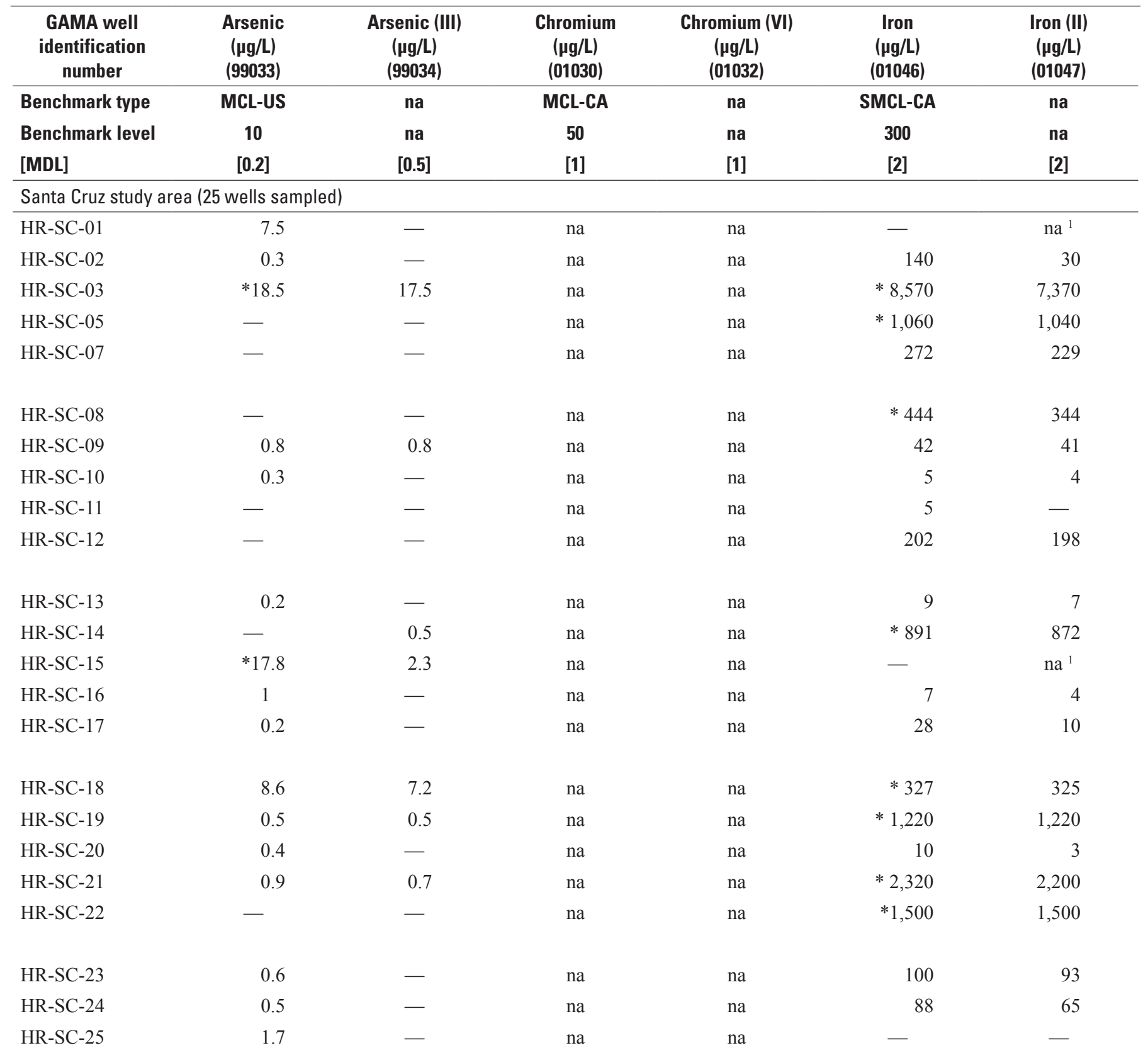


Table 11. Arsenic, chromium, and iron species detected in samples collected for the Santa Cruz, San Gabriel, and Peninsular Ranges Hard Rock Aquifers (Hard Rock) study unit, March 2011 through March 2012, California GAMA Priority Basin Project.—Continued

[The five-digit U.S. Geological Survey (USGS) parameter code below the constituent name is used to uniquely identify a specific constituent or property. Samples from 110 wells were analyzed for arsenic and iron species. Samples from all 37 San Gabriel study area wells were analyzed for chromium species. Only samples with detections of at least one constituent are listed. GAMA well identification number: HR-SC, Santa Cruz study area grid well; HR-SG, San Gabriel study area grid well; HR-SGU, San Gabriel study area understanding well; HR-PR, Peninsular Ranges study area grid well; HR-PRFP, Peninsular Ranges study area flow-path well; HR-PRU, Peninsular Ranges study area understanding well. Reporting levels, benchmark types, and benchmark levels as of July 6, 2012. Benchmark type: Maximum contaminant level benchmarks are listed as MCL-US when the MCL-US and MCL-CA are identical, and as MCL-CA when the MCL-CA is lower than the MCL-US or no MCL-US exists. Secondary maximum contaminant level benchmarks are listed as SMCL-CA when the SMCL-CA exists, and as the SMCL-US when the SMCL-CA does not exist. MCL-US, U.S. Environmental Protection Agency maximum contaminant level; MCL-CA, California Department of Public Health maximum contaminant level; SMCL-CA, California Department of Public Health secondary maximum contaminant level. Other abbreviations: MDL, method detection limit; $\mu \mathrm{g} / \mathrm{L}$, micrograms per liter; —, not detected; na, not available; *, greater than the benchmark level]

\begin{tabular}{|c|c|c|c|c|c|c|}
\hline $\begin{array}{l}\text { GAMA well } \\
\text { identification } \\
\text { number }\end{array}$ & $\begin{array}{c}\text { Arsenic } \\
(\mu \mathrm{g} / \mathrm{L}) \\
(99033)\end{array}$ & $\begin{array}{c}\text { Arsenic (III) } \\
(\mu \mathrm{g} / \mathrm{L}) \\
(99034)\end{array}$ & $\begin{array}{c}\text { Chromium } \\
(\mu \mathrm{g} / \mathrm{L}) \\
(01030)\end{array}$ & $\begin{array}{c}\text { Chromium (VI) } \\
(\mu \mathrm{g} / \mathrm{L}) \\
(01032)\end{array}$ & $\begin{array}{c}\text { Iron } \\
(\mu \mathrm{g} / \mathrm{L}) \\
(01046)\end{array}$ & $\begin{array}{c}\text { Iron (II) } \\
(\mu \mathrm{g} / \mathrm{L}) \\
(01047)\end{array}$ \\
\hline Benchmark type & MCL-US & na & MCL-CA & na & SMCL-CA & na \\
\hline Benchmark level & 10 & na & 50 & na & 300 & na \\
\hline [MDL] & {$[0.2]$} & [0.5] & [1] & [1] & [2] & [2] \\
\hline \multicolumn{7}{|c|}{ San Gabriel study area (37 wells sampled) } \\
\hline HR-SG-01 & $\mathrm{na}^{2}$ & $\mathrm{na}^{2}$ & - & - & $\mathrm{na}^{2}$ & $\mathrm{na}^{2}$ \\
\hline HR-SG-02 & - & - & - & - & 86 & 82 \\
\hline HR-SG-03 & 0.5 & - & - & - & - & - \\
\hline HR-SG-04 & 0.3 & - & - & - & 5 & - \\
\hline HR-SG-05 & - & - & - & - & 5 & - \\
\hline HR-SG-06 & 4.3 & - & - & - & 3 & - \\
\hline HR-SG-07 & 0.6 & - & - & - & $* 473$ & 245 \\
\hline HR-SG-08 & 0.8 & - & - & - & 34 & 26 \\
\hline HR-SG-09 & * 14.1 & 2.2 & - & - & 15 & 4 \\
\hline HR-SG-10 & - & - & - & - & 4 & - \\
\hline HR-SG-11 & - & - & - & - & 7 & 2 \\
\hline HR-SG-12 & 0.5 & - & - & - & - & - \\
\hline HR-SG-13 & - & - & - & - & 7 & 5 \\
\hline HR-SG-14 & - & - & - & - & $* 471$ & 455 \\
\hline HR-SG-15 & - & - & - & - & 4 & - \\
\hline HR-SG-16 & 2.2 & - & - & - & - & - \\
\hline HR-SG-17 & - & - & - & - & 3 & - \\
\hline HR-SG-18 & 0.5 & - & - & - & 3 & 3 \\
\hline HR-SG-19 & - & - & - & - & 7 & 5 \\
\hline HR-SG-20 & - & - & - & - & 5 & 3 \\
\hline HR-SG-21 & - & - & - & - & 87 & 38 \\
\hline HR-SG-22 & 1.1 & - & - & - & 3 & 3 \\
\hline HR-SG-23 & 0.2 & - & - & - & - & - \\
\hline HR-SG-24 & 2.3 & 0.8 & - & - & 5 & - \\
\hline HR-SG-25 & 0.3 & - & - & - & 6 & 4 \\
\hline
\end{tabular}


Table 11. Arsenic, chromium, and iron species detected in samples collected for the Santa Cruz, San Gabriel, and Peninsular Ranges Hard Rock Aquifers (Hard Rock) study unit, March 2011 through March 2012, California GAMA Priority Basin Project.—Continued

[The five-digit U.S. Geological Survey (USGS) parameter code below the constituent name is used to uniquely identify a specific constituent or property. Samples from 110 wells were analyzed for arsenic and iron species. Samples from all 37 San Gabriel study area wells were analyzed for chromium species. Only samples with detections of at least one constituent are listed. GAMA well identification number: HR-SC, Santa Cruz study area grid well; HR-SG, San Gabriel study area grid well; HR-SGU, San Gabriel study area understanding well; HR-PR, Peninsular Ranges study area grid well; HR-PRFP, Peninsular Ranges study area flow-path well; HR-PRU, Peninsular Ranges study area understanding well. Reporting levels, benchmark types, and benchmark levels as of July 6, 2012. Benchmark type: Maximum contaminant level benchmarks are listed as MCL-US when the MCL-US and MCL-CA are identical, and as MCL-CA when the MCL-CA is lower than the MCL-US or no MCL-US exists. Secondary maximum contaminant level benchmarks are listed as SMCL-CA when the SMCL-CA exists, and as the SMCL-US when the SMCL-CA does not exist. MCL-US, U.S. Environmental Protection Agency maximum contaminant level; MCL-CA, California Department of Public Health maximum contaminant level; SMCL-CA, California Department of Public Health secondary maximum contaminant level. Other abbreviations: MDL, method detection limit; $\mu \mathrm{g} / \mathrm{L}$, micrograms per liter; -, not detected; na, not available; *, greater than the benchmark level]

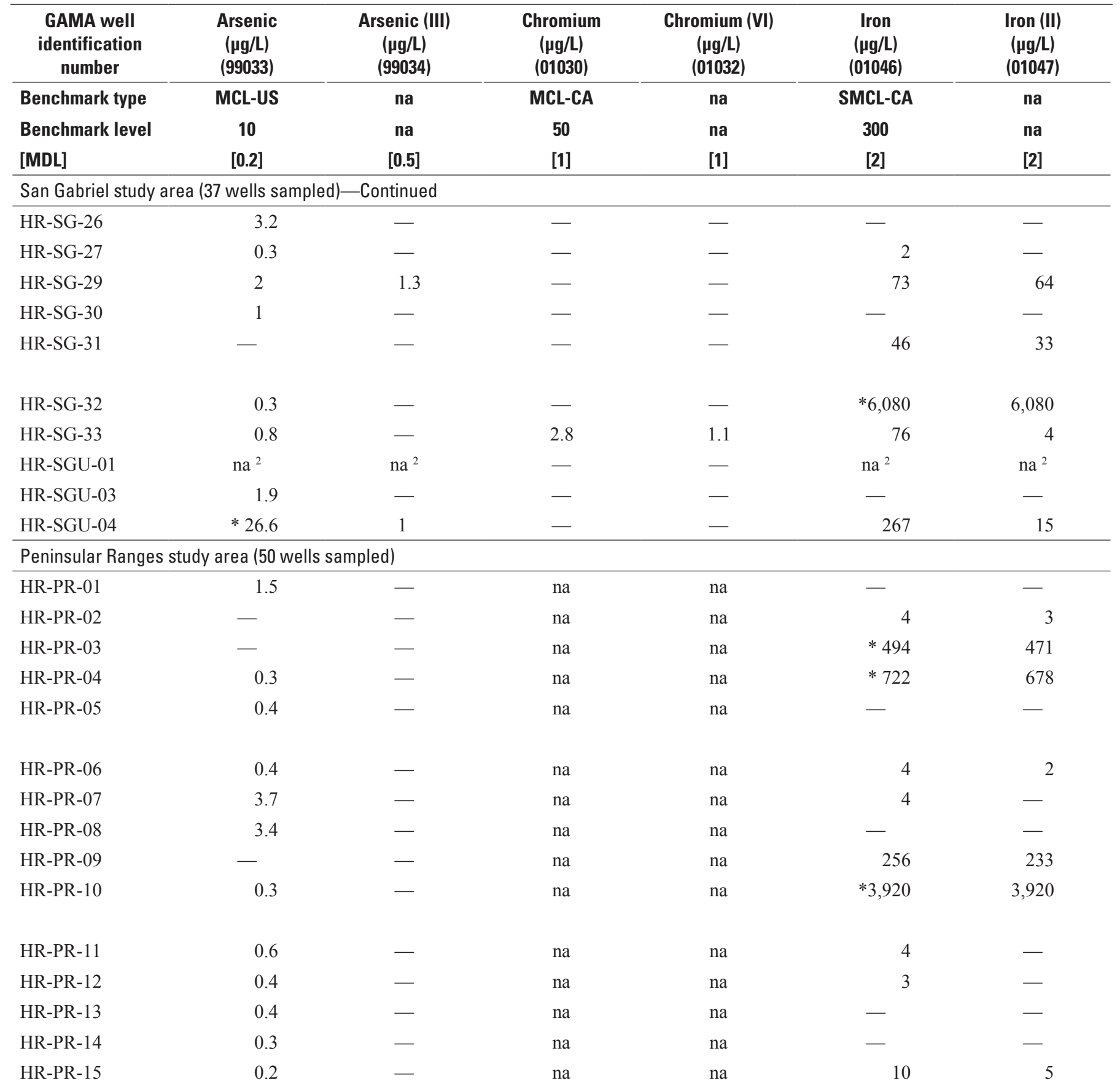


Table 11. Arsenic, chromium, and iron species detected in samples collected for the Santa Cruz, San Gabriel, and Peninsular Ranges Hard Rock Aquifers (Hard Rock) study unit, March 2011 through March 2012, California GAMA Priority Basin Project._Continued

[The five-digit U.S. Geological Survey (USGS) parameter code below the constituent name is used to uniquely identify a specific constituent or property. Samples from 110 wells were analyzed for arsenic and iron species. Samples from all 37 San Gabriel study area wells were analyzed for chromium species. Only samples with detections of at least one constituent are listed. GAMA well identification number: HR-SC, Santa Cruz study area grid well; HR-SG, San Gabriel study area grid well; HR-SGU, San Gabriel study area understanding well; HR-PR, Peninsular Ranges study area grid well; HR-PRFP, Peninsular Ranges study area flow-path well; HR-PRU, Peninsular Ranges study area understanding well. Reporting levels, benchmark types, and benchmark levels as of July 6, 2012. Benchmark type: Maximum contaminant level benchmarks are listed as MCL-US when the MCL-US and MCL-CA are identical, and as MCL-CA when the MCL-CA is lower than the MCL-US or no MCL-US exists. Secondary maximum contaminant level benchmarks are listed as SMCL-CA when the SMCL-CA exists, and as the SMCL-US when the SMCL-CA does not exist. MCL-US, U.S. Environmental Protection Agency maximum contaminant level; MCL-CA, California Department of Public Health maximum contaminant level; SMCL-CA, California Department of Public Health secondary maximum contaminant level. Other abbreviations: MDL, method detection limit; $\mu \mathrm{g} / \mathrm{L}$, micrograms per liter; —, not detected; na, not available; *, greater than the benchmark level]

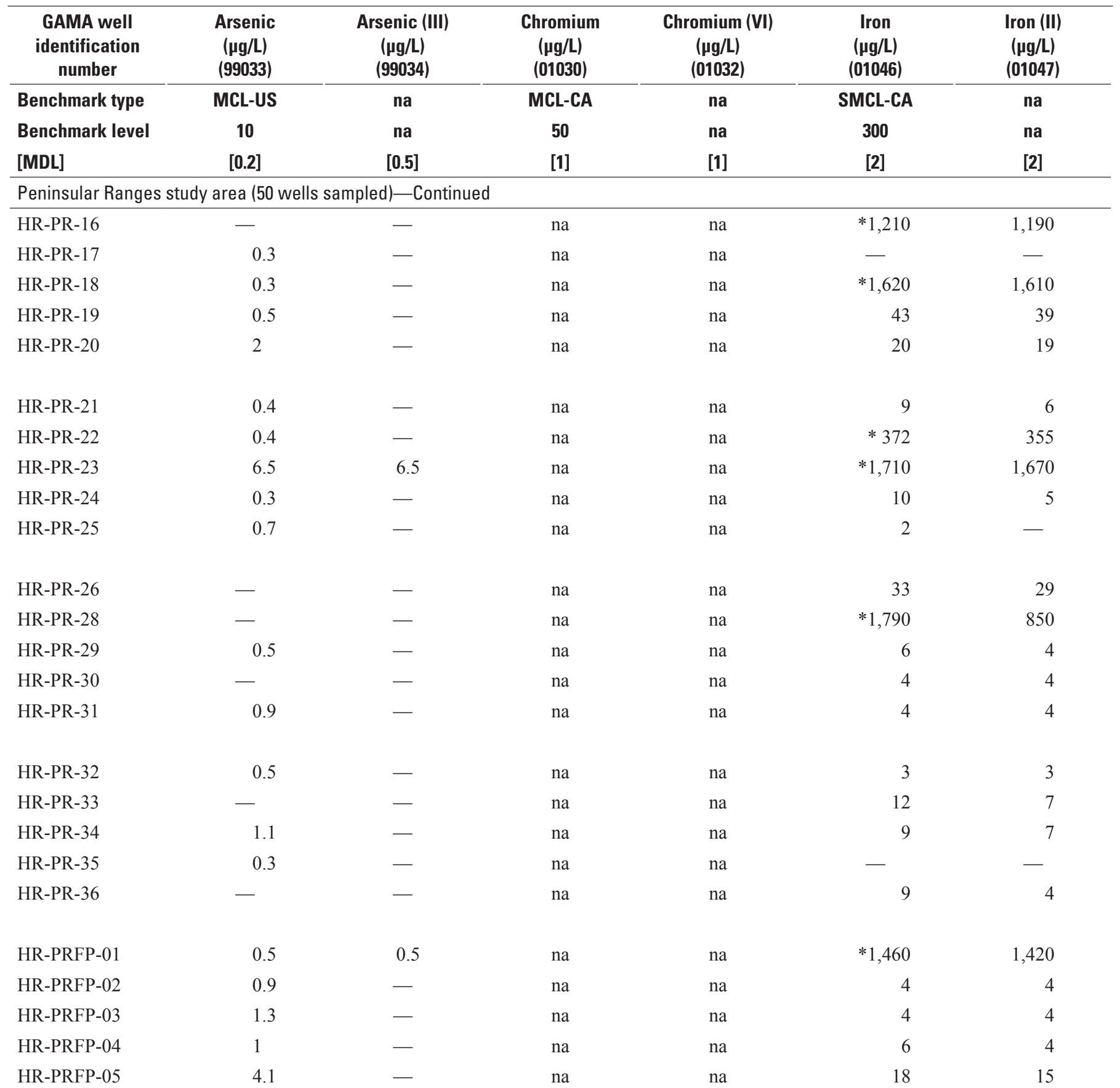


Table 11. Arsenic, chromium, and iron species detected in samples collected for the Santa Cruz, San Gabriel, and Peninsular Ranges Hard Rock Aquifers (Hard Rock) study unit, March 2011 through March 2012, California GAMA Priority Basin Project.—Continued

[The five-digit U.S. Geological Survey (USGS) parameter code below the constituent name is used to uniquely identify a specific constituent or property. Samples from 110 wells were analyzed for arsenic and iron species. Samples from all 37 San Gabriel study area wells were analyzed for chromium species. Only samples with detections of at least one constituent are listed. GAMA well identification number: HR-SC, Santa Cruz study area grid well; HR-SG, San Gabriel study area grid well; HR-SGU, San Gabriel study area understanding well; HR-PR, Peninsular Ranges study area grid well; HR-PRFP, Peninsular Ranges study area flow-path well; HR-PRU, Peninsular Ranges study area understanding well. Reporting levels, benchmark types, and benchmark levels as of July 6, 2012. Benchmark type: Maximum contaminant level benchmarks are listed as MCL-US when the MCL-US and MCL-CA are identical, and as MCL-CA when the MCL-CA is lower than the MCL-US or no MCL-US exists. Secondary maximum contaminant level benchmarks are listed as SMCL-CA when the SMCL-CA exists, and as the SMCL-US when the SMCL-CA does not exist. MCL-US, U.S. Environmental Protection Agency maximum contaminant level; MCL-CA, California Department of Public Health maximum contaminant level; SMCL-CA, California Department of Public Health secondary maximum contaminant level. Other abbreviations: MDL, method detection limit; $\mu \mathrm{g} / \mathrm{L}$, micrograms per liter; 一, not detected; na, not available; *, greater than the benchmark level]

\begin{tabular}{|c|c|c|c|c|c|c|}
\hline $\begin{array}{l}\text { GAMA well } \\
\text { identification } \\
\text { number }\end{array}$ & $\begin{array}{c}\text { Arsenic } \\
(\mu g / L) \\
(99033)\end{array}$ & $\begin{array}{c}\text { Arsenic (III) } \\
(\mu \mathrm{g} / \mathrm{L}) \\
(99034)\end{array}$ & $\begin{array}{c}\text { Chromium } \\
(\mu \mathrm{g} / \mathrm{L}) \\
(01030)\end{array}$ & $\begin{array}{c}\text { Chromium (VI) } \\
(\mu \mathrm{g} / \mathrm{L}) \\
(01032)\end{array}$ & $\begin{array}{c}\text { Iron } \\
(\mu \mathrm{g} / \mathrm{L}) \\
(01046)\end{array}$ & $\begin{array}{c}\text { Iron (II) } \\
(\mu \mathrm{g} / \mathrm{L}) \\
(01047)\end{array}$ \\
\hline Benchmark type & MCL-US & na & MCL-CA & na & SMCL-CA & na \\
\hline Benchmark level & 10 & na & 50 & na & 300 & na \\
\hline [MDL] & [0.2] & {$[0.5]$} & [1] & [1] & [2] & [2] \\
\hline \multicolumn{7}{|c|}{ Peninsular Ranges study area (50 wells sampled)—Continued } \\
\hline HR-PRFP-06 & $* 12.8$ & 8.4 & na & na & 132 & 127 \\
\hline HR-PRFP-07 & - & - & na & na & * 480 & 469 \\
\hline HR-PRFP-08 & - & - & na & na & 2 & 2 \\
\hline HR-PRFP-09 & 1.8 & - & na & na & - & - \\
\hline HR-PRFP-10 & 0.4 & - & na & na & 176 & 148 \\
\hline HR-PRFP-11 & 0.6 & - & na & na & 6 & 5 \\
\hline HR-PRFP-12 & 0.3 & - & na & na & - & - \\
\hline HR-PRFP-13 & 5.2 & - & na & na & 240 & 9 \\
\hline HR-PRU-01 & 0.3 & - & na & na & 13 & 3 \\
\hline
\end{tabular}

${ }^{1}$ Iron (II) is frequently not measured if iron (total) is not detected.

${ }^{2}$ HR-SG-01 and HR-SGU-01 samples were not analyzed for arsenic and iron speciation due to sample mix-up. 
Table 12. Results for analyses of stable isotope ratios, carbon-14 abundance, and tritium activity in samples collected for the Santa Cruz, San Gabriel, and Peninsular Ranges Hard Rock Aquifers (Hard Rock) study unit, March 2011 through March 2012, California GAMA Priority Basin Project.

[The five-digit U.S. Geological Survey (USGS) parameter code below the constituent name is used to uniquely identify a specific constituent or property. Stable isotope ratios are reported in the standard delta notation $(\delta)$, the ratio of a heavier isotope to more common lighter isotope of that element, relative to a standard reference material. Measured values of tritium less than the sample-specific critical level ( $\left.\mathrm{ssL}_{\mathrm{C}}\right)$ are reported as not detected (-). GAMA well identification number: HR-SC, Santa Cruz study area grid well; HR-SG, San Gabriel study area grid well; HR-SGU, San Gabriel study area understanding well; HR-PR, Peninsular Ranges study area grid well; HR-PRFP, Peninsular Ranges study area flow-path well; HR-PRU, Peninsular Ranges study area understanding well. Reporting levels, benchmark types, and benchmark levels as of July 6, 2012. Benchmark type: Maximum contaminant level benchmarks are listed as MCL-US when the MCL-US and MCL-CA are identical, and as MCL-CA when the MCL-CA is lower than the MCL-US or no MCL-US exists. MCL-CA, California Department of Public Health maximum contaminant level. Elements: H, hydrogen; O, oxygen; C, carbon. Other abbreviations: CSU, 1-sigma combined standard uncertainty; $\mathrm{ssL}_{\mathrm{C}}$, sample-specific critical level; \pm , plus or minus; $\mathrm{pCi} / \mathrm{L}$, picocuries per liter; na, not available]

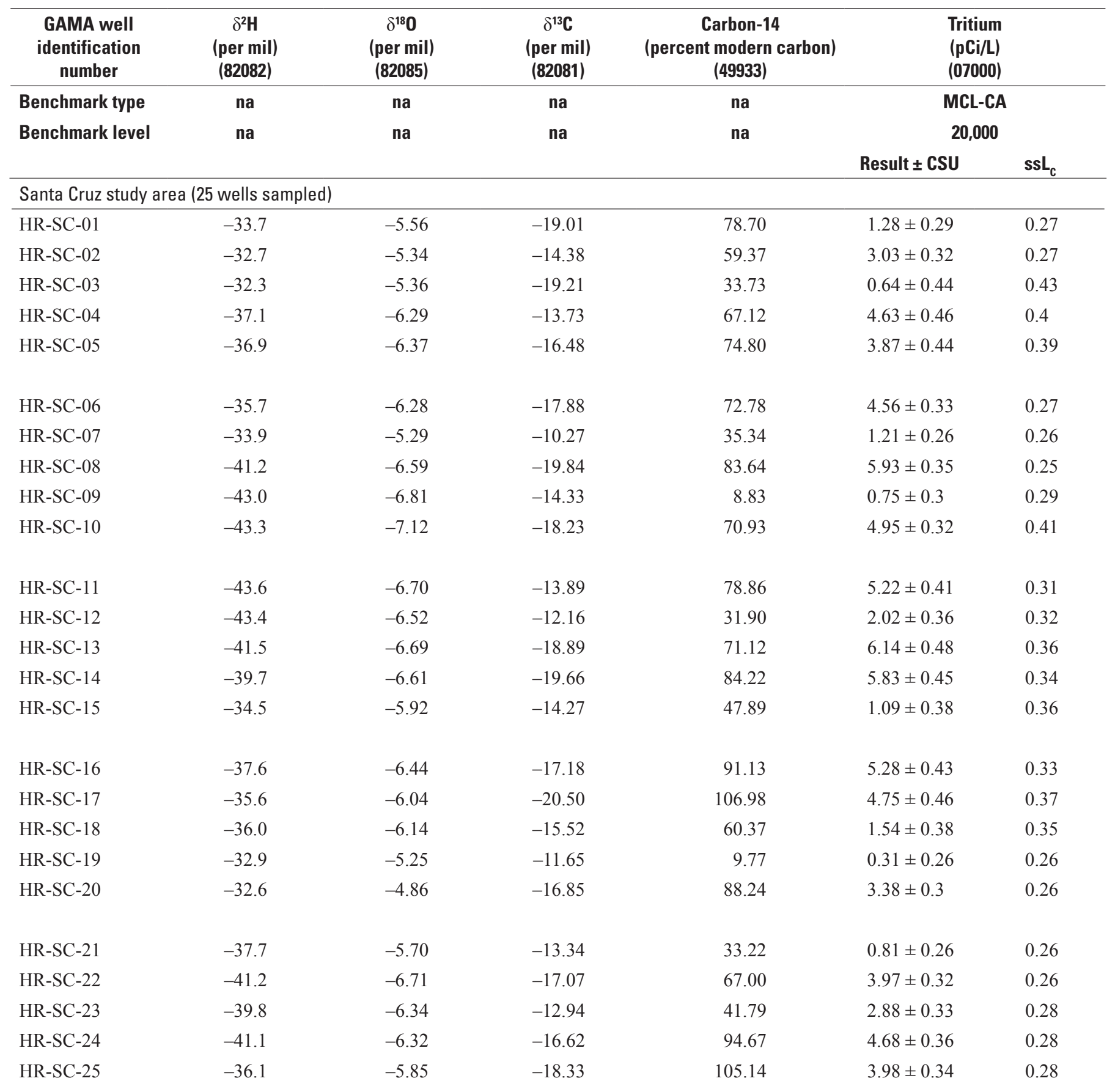


Table 12. Results for analyses of stable isotope ratios, carbon-14 abundance, and tritium activity in samples collected for the Santa Cruz, San Gabriel, and Peninsular Ranges Hard Rock Aquifers (Hard Rock) study unit, March 2011 through March 2012, California GAMA Priority Basin Project.-Continued

[The five-digit U.S. Geological Survey (USGS) parameter code below the constituent name is used to uniquely identify a specific constituent or property. Stable isotope ratios are reported in the standard delta notation $(\delta)$, the ratio of a heavier isotope to more common lighter isotope of that element, relative to a standard reference material. Measured values of tritium less than the sample-specific critical level $\left(\mathrm{ssL}_{C}\right)$ are reported as not detected ( - ). GAMA well identification number: HR-SC, Santa Cruz study area grid well; HR-SG, San Gabriel study area grid well; HR-SGU, San Gabriel study area understanding well; HR-PR, Peninsular Ranges study area grid well; HR-PRFP, Peninsular Ranges study area flow-path well; HR-PRU, Peninsular Ranges study area understanding well. Reporting levels, benchmark types, and benchmark levels as of July 6, 2012. Benchmark type: Maximum contaminant level benchmarks are listed as MCL-US when the MCL-US and MCL-CA are identical, and as MCL-CA when the MCL-CA is lower than the MCL-US or no MCL-US exists. MCL-CA, California Department of Public Health maximum contaminant level. Elements: H, hydrogen; O, oxygen; C, carbon. Other abbreviations: CSU, 1-sigma combined standard uncertainty; $\mathrm{ssL}_{\mathrm{C}}$, sample-specific critical level; \pm , plus or minus; $\mathrm{pCi} / \mathrm{L}$, picocuries per liter; na, not available]

\begin{tabular}{|c|c|c|c|c|c|c|}
\hline $\begin{array}{l}\text { GAMA well } \\
\text { identification } \\
\text { number }\end{array}$ & $\begin{array}{c}\delta^{2} \mathrm{H} \\
\text { (per mil) } \\
(82082)\end{array}$ & $\begin{array}{c}\delta^{180} \\
\text { (per mil) } \\
(82085)\end{array}$ & $\begin{array}{c}\delta^{13} \mathrm{C} \\
\text { (per mil) } \\
(82081)\end{array}$ & $\begin{array}{c}\text { Carbon-14 } \\
\text { (percent modern carbon) } \\
\text { (49933) }\end{array}$ & \multicolumn{2}{|c|}{$\begin{array}{l}\text { Tritium } \\
\text { (pCi/L) } \\
\text { (07000) }\end{array}$} \\
\hline Benchmark type & na & na & na & na & \multicolumn{2}{|c|}{ MCL-CA } \\
\hline \multirow[t]{2}{*}{ Benchmark level } & \multirow[t]{2}{*}{ na } & \multirow[t]{2}{*}{ na } & \multirow[t]{2}{*}{ na } & \multirow[t]{2}{*}{ na } & \multicolumn{2}{|c|}{20,000} \\
\hline & & & & & Result \pm CSU & ssL $_{c}$ \\
\hline HR-SG-02 & -55.9 & -8.51 & -14.61 & 104.04 & $7.59 \pm 0.5$ & 0.34 \\
\hline HR-SG-03 & -59.8 & -8.35 & -14.40 & 103.94 & $9.83 \pm 0.54$ & 0.32 \\
\hline HR-SG-04 & -58.3 & -8.65 & -14.03 & 96.43 & $3.57 \pm 0.38$ & 0.31 \\
\hline HR-SG-05 & -60.2 & -8.94 & -13.40 & 103.11 & $8.06 \pm 0.51$ & 0.34 \\
\hline HR-SG-08 & -60.3 & -8.20 & -15.81 & 104.04 & $6.93 \pm 0.45$ & 0.31 \\
\hline HR-SG-09 & -54.6 & -7.65 & -11.33 & 60.03 & $0.64 \pm 0.31$ & 0.3 \\
\hline HR-SG-10 & -65.6 & -9.61 & -14.89 & 102.93 & $5.61 \pm 0.33$ & 0.25 \\
\hline HR-SG-11 & -67.0 & -9.97 & -15.40 & 83.10 & - & 0.27 \\
\hline HR-SG-12 & -57.5 & -8.86 & -17.68 & 89.20 & $6.44 \pm 0.39$ & 0.28 \\
\hline HR-SG-13 & -76.7 & -11.14 & -14.74 & 80.00 & $5.03 \pm 0.33$ & 0.25 \\
\hline HR-SG-14 & -74.2 & -10.96 & -16.16 & 77.51 & $10.19 \pm 0.63$ & 0.38 \\
\hline HR-SG-15 & -69.8 & -10.45 & -15.60 & 95.83 & $13.74 \pm 0.76$ & 0.41 \\
\hline HR-SG-21 & -63.8 & -9.62 & -17.23 & 94.76 & $5.3 \pm 0.53$ & 0.41 \\
\hline HR-SG-22 & -98.0 & -12.58 & -12.08 & 91.40 & $11.67 \pm 0.66$ & 0.36 \\
\hline HR-SG-23 & -60.8 & -9.30 & -12.56 & 94.22 & $8.67 \pm 0.59$ & 0.38 \\
\hline HR-SG-24 & -50.4 & -7.76 & -15.60 & 101.75 & $8.47 \pm 0.59$ & 0.38 \\
\hline HR-SG-25 & -49.6 & -7.92 & -19.82 & 95.70 & $3.55 \pm 0.45$ & 0.37 \\
\hline
\end{tabular}


Table 12. Results for analyses of stable isotope ratios, carbon-14 abundance, and tritium activity in samples collected for the Santa Cruz, San Gabriel, and Peninsular Ranges Hard Rock Aquifers (Hard Rock) study unit, March 2011 through March 2012, California GAMA Priority Basin Project.-Continued

[The five-digit U.S. Geological Survey (USGS) parameter code below the constituent name is used to uniquely identify a specific constituent or property. Stable isotope ratios are reported in the standard delta notation $(\delta)$, the ratio of a heavier isotope to more common lighter isotope of that element, relative to a standard reference material. Measured values of tritium less than the sample-specific critical level ( $\left.\mathrm{ssL}_{\mathrm{C}}\right)$ are reported as not detected (-). GAMA well identification number: HR-SC, Santa Cruz study area grid well; HR-SG, San Gabriel study area grid well; HR-SGU, San Gabriel study area understanding well; HR-PR, Peninsular Ranges study area grid well; HR-PRFP, Peninsular Ranges study area flow-path well; HR-PRU, Peninsular Ranges study area understanding well. Reporting levels, benchmark types, and benchmark levels as of July 6, 2012. Benchmark type: Maximum contaminant level benchmarks are listed as MCL-US when the MCL-US and MCL-CA are identical, and as MCL-CA when the MCL-CA is lower than the MCL-US or no MCL-US exists. MCL-CA, California Department of Public Health maximum contaminant level. Elements: H, hydrogen; O, oxygen; C, carbon. Other abbreviations: CSU, 1-sigma combined standard uncertainty; $\mathrm{ssL}_{\mathrm{C}}$, sample-specific critical level; \pm , plus or minus; $\mathrm{pCi} / \mathrm{L}$, picocuries per liter; na, not available]

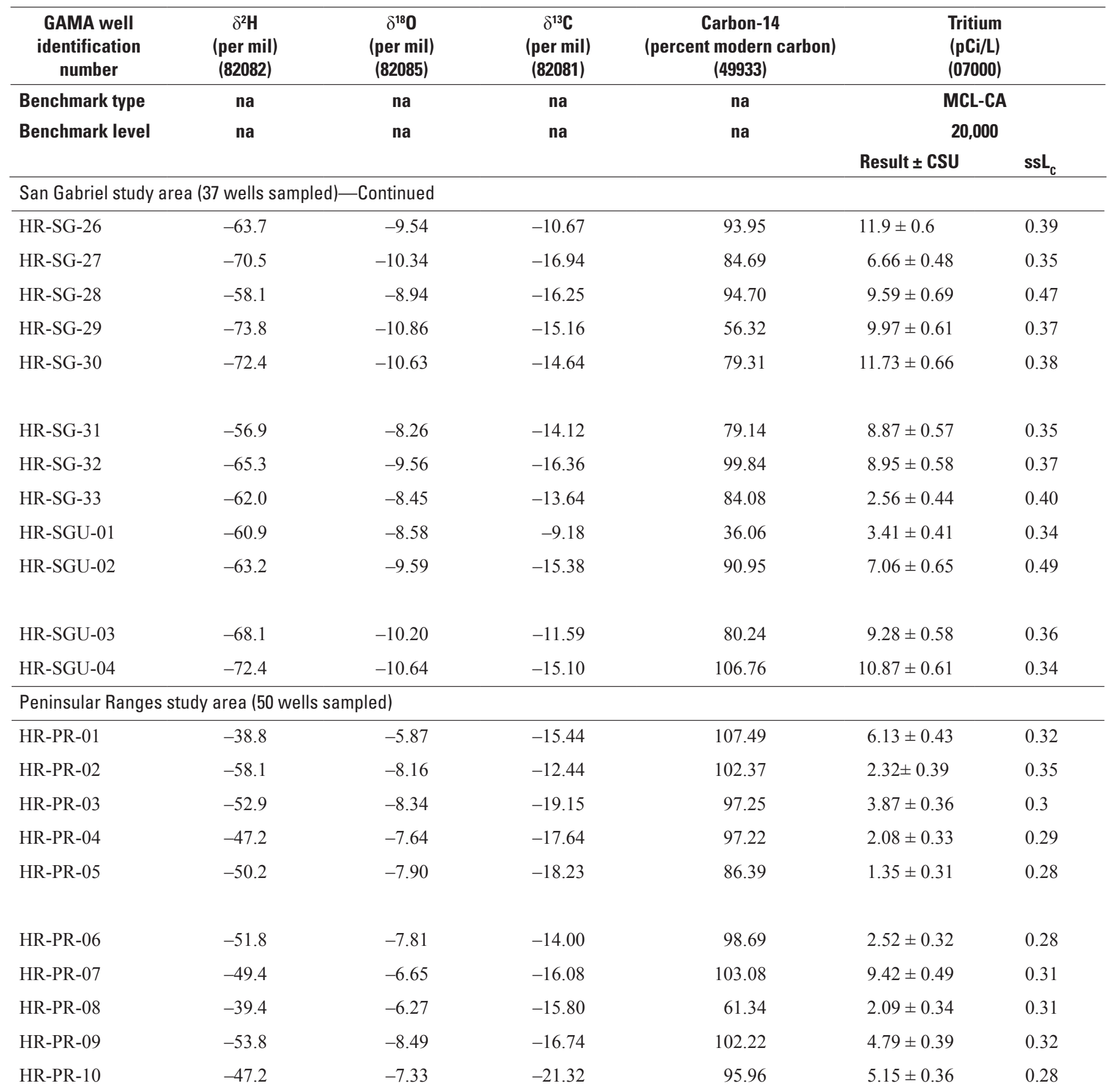


Table 12. Results for analyses of stable isotope ratios, carbon-14 abundance, and tritium activity in samples collected for the Santa Cruz, San Gabriel, and Peninsular Ranges Hard Rock Aquifers (Hard Rock) study unit, March 2011 through March 2012, California GAMA Priority Basin Project.-Continued

[The five-digit U.S. Geological Survey (USGS) parameter code below the constituent name is used to uniquely identify a specific constituent or property. Stable isotope ratios are reported in the standard delta notation $(\delta)$, the ratio of a heavier isotope to more common lighter isotope of that element, relative to a standard reference material. Measured values of tritium less than the sample-specific critical level $\left(\mathrm{ssL}_{\mathrm{C}}\right)$ are reported as not detected (一). GAMA well identification number: HR-SC, Santa Cruz study area grid well; HR-SG, San Gabriel study area grid well; HR-SGU, San Gabriel study area understanding well; HR-PR, Peninsular Ranges study area grid well; HR-PRFP, Peninsular Ranges study area flow-path well; HR-PRU, Peninsular Ranges study area understanding well. Reporting levels, benchmark types, and benchmark levels as of July 6, 2012. Benchmark type: Maximum contaminant level benchmarks are listed as MCL-US when the MCL-US and MCL-CA are identical, and as MCL-CA when the MCL-CA is lower than the MCL-US or no MCL-US exists. MCL-CA, California Department of Public Health maximum contaminant level. Elements: H, hydrogen; O, oxygen; C, carbon. Other abbreviations: CSU, 1-sigma combined standard uncertainty; $\mathrm{ssL}_{\mathrm{C}}$, sample-specific critical level; \pm , plus or minus; $\mathrm{pCi} / \mathrm{L}$, picocuries per liter; na, not available]

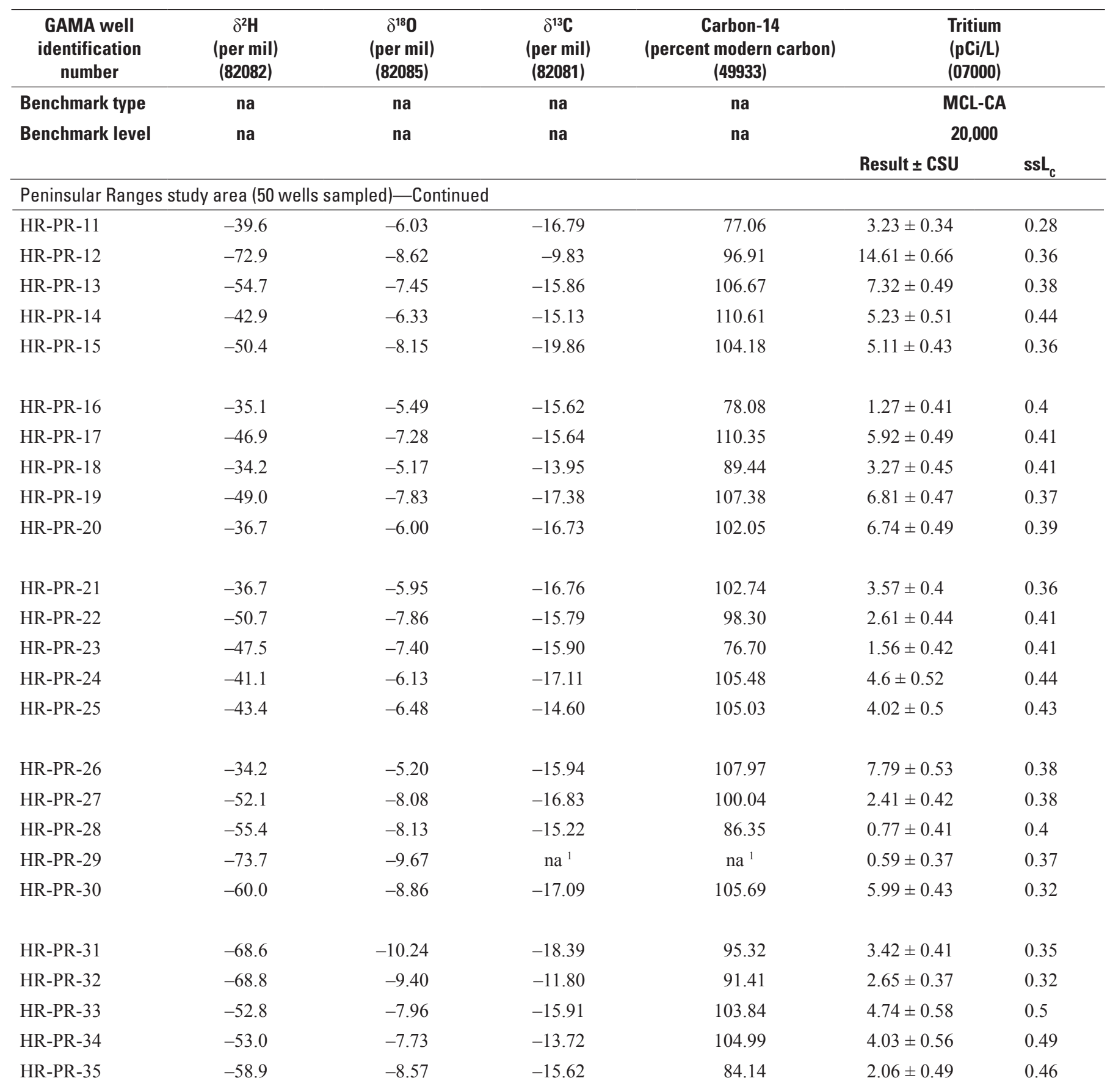


Table 12. Results for analyses of stable isotope ratios, carbon-14 abundance, and tritium activity in samples collected for the Santa Cruz, San Gabriel, and Peninsular Ranges Hard Rock Aquifers (Hard Rock) study unit, March 2011 through March 2012, California GAMA Priority Basin Project.-Continued

[The five-digit U.S. Geological Survey (USGS) parameter code below the constituent name is used to uniquely identify a specific constituent or property. Stable isotope ratios are reported in the standard delta notation $(\delta)$, the ratio of a heavier isotope to more common lighter isotope of that element, relative to a standard reference material. Measured values of tritium less than the sample-specific critical level ( $\left.\mathrm{ssL}_{\mathrm{C}}\right)$ are reported as not detected (-). GAMA well identification number: HR-SC, Santa Cruz study area grid well; HR-SG, San Gabriel study area grid well; HR-SGU, San Gabriel study area understanding well; HR-PR, Peninsular Ranges study area grid well; HR-PRFP, Peninsular Ranges study area flow-path well; HR-PRU, Peninsular Ranges study area understanding well. Reporting levels, benchmark types, and benchmark levels as of July 6, 2012. Benchmark type: Maximum contaminant level benchmarks are listed as MCL-US when the MCL-US and MCL-CA are identical, and as MCL-CA when the MCL-CA is lower than the MCL-US or no MCL-US exists. MCL-CA, California Department of Public Health maximum contaminant level. Elements: H, hydrogen; O, oxygen; C, carbon. Other abbreviations: CSU, 1-sigma combined standard uncertainty; $\mathrm{ssL}_{\mathrm{C}}$, sample-specific critical level; \pm , plus or minus; $\mathrm{pCi} / \mathrm{L}$, picocuries per liter; na, not available]

\begin{tabular}{|c|c|c|c|c|c|c|}
\hline $\begin{array}{c}\text { GAMA well } \\
\text { identification } \\
\text { number }\end{array}$ & $\begin{array}{c}\delta^{2} \mathrm{H} \\
\text { (per mil) } \\
(82082)\end{array}$ & $\begin{array}{c}\delta^{180} \\
\text { (per mil) } \\
(82085)\end{array}$ & $\begin{array}{c}\delta^{13} \mathrm{C} \\
\text { (per mil) } \\
(82081)\end{array}$ & $\begin{array}{c}\text { Carbon-14 } \\
\text { (percent modern carbon) } \\
\text { (49933) }\end{array}$ & \multicolumn{2}{|c|}{$\begin{array}{l}\text { Tritium } \\
\text { (pCi/L) } \\
(07000)\end{array}$} \\
\hline Benchmark type & na & na & na & na & \multicolumn{2}{|c|}{ MCL-CA } \\
\hline Benchmark level & na & na & na & na & \multicolumn{2}{|c|}{20,000} \\
\hline & & & & & Result \pm CSU & ssL $_{c}$ \\
\hline \multicolumn{7}{|c|}{ Peninsular Ranges study area (50 wells sampled)_Continued } \\
\hline HR-PRFP-01 & -49.1 & -7.58 & -16.07 & 72.67 & $0.67 \pm 0.48$ & 0.47 \\
\hline HR-PRFP-02 & -51.6 & -6.85 & -17.37 & 97.44 & $8.08 \pm 0.62$ & 0.47 \\
\hline HR-PRFP-03 & -46.8 & -7.37 & -17.98 & 58.49 & $1.28 \pm 0.48$ & 0.46 \\
\hline HR-PRFP-04 & -38.6 & -6.02 & -17.15 & 92.69 & $2.44 \pm 0.5$ & 0.46 \\
\hline HR-PRFP-07 & -51.8 & -8.11 & -16.51 & 99.39 & $5.25 \pm 0.55$ & 0.46 \\
\hline HR-PRFP-08 & -57.0 & -8.92 & -17.85 & 43.08 & $0.93 \pm 0.35$ & 0.33 \\
\hline HR-PRFP-09 & -44.8 & -6.08 & -16.97 & 103.39 & $7.29 \pm 0.45$ & 0.31 \\
\hline HR-PRFP-10 & -47.3 & -7.48 & $\mathrm{na}^{1}$ & $\mathrm{na}^{1}$ & $1.00 \pm 0.37$ & 0.35 \\
\hline HR-PRFP-11 & -34.1 & -5.24 & -15.58 & 93.70 & $2.55 \pm 0.27$ & 0.24 \\
\hline HR-PRFP-12 & -37.1 & -5.60 & -14.85 & 106.25 & $4.96 \pm 0.27$ & 0.18 \\
\hline HR-PRFP-13 & -41.1 & -6.16 & -17.02 & 104.79 & $8.18 \pm 0.39$ & 0.23 \\
\hline HR-PRU-01 & -52.1 & -8.41 & -18.54 & 98.45 & $1.99 \pm 0.44$ & 0.38 \\
\hline
\end{tabular}

${ }^{1}$ No results for samples HR-PR-29 (valve malfunction during analysis) and HR-PRFP-10 (broken during storage). 
Table 13. Results for analyses of dissolved noble gases by the Lawrence Livermore National Laboratory (LLNL) for samples collected for the Santa Cruz, San Gabriel, and Peninsular Ranges Hard Rock Aquifers (Hard Rock) study unit, March 2011 through March 2012, California GAMA Priority Basin Project.

[Results for noble gas analyses from the LLNL are stored in a separate database maintained by the GAMA Program. Information about the analytes given in table 3I. GAMA well identification number: HR-SC, Santa Cruz study area grid well; HR-SG, San Gabriel study area grid well; HR-SGU, San Gabriel study area understanding well; HR-PR, Peninsular Ranges study area grid well; HR-PRFP, Peninsular Ranges study area flow-path well; HR-PRU, Peninsular Ranges study area understanding well. Other abbreviations: $\mathrm{cm}^{3} \mathrm{STP} / \mathrm{g}$, cubic centimeters per gram at standard temperature and pressure]

\begin{tabular}{|c|c|c|c|c|c|c|}
\hline $\begin{array}{c}\text { GAMA well } \\
\text { identification number }\end{array}$ & $\begin{array}{c}\text { Argon } \\
\left(10^{4} \mathrm{~cm}^{3} \mathrm{STP} / \mathrm{g}\right)\end{array}$ & $\begin{array}{l}\text { Helium-3/Helium-4 } \\
\text { (10 atom ratio) }\end{array}$ & $\begin{array}{c}\text { Helium-4 } \\
\left(10^{8} \mathrm{~cm}^{3} \mathrm{STP} / \mathrm{g}\right)\end{array}$ & $\begin{array}{c}\text { Krypton } \\
\left(10^{8} \mathrm{~cm}^{3} \mathrm{STP} / \mathrm{g}\right)\end{array}$ & $\begin{array}{c}\text { Neon } \\
\left(10^{7} \mathrm{~cm}^{3} \mathrm{STP} / \mathrm{g}\right)\end{array}$ & $\begin{array}{c}\text { Xenon } \\
\left(10^{8} \mathrm{~cm}^{3} S T P / g\right)\end{array}$ \\
\hline \multicolumn{7}{|c|}{ Santa Cruz study area ( 23 wells sampled) ${ }^{1}$} \\
\hline HR-SC-02 & 3.756 & 1.43 & 5.85 & 8.46 & 2.44 & 1.17 \\
\hline HR-SC-03 & 9.632 & 1.39 & 28.4 & 18.73 & 10.29 & 2.12 \\
\hline HR-SC-06 & 3.62 & 1.37 & 4.57 & 8.51 & 1.97 & 1.23 \\
\hline HR-SC-07 & 4.06 & 1.32 & 6.06 & 8.85 & 2.60 & 1.29 \\
\hline HR-SC-08 & 4.35 & 1.41 & 8.96 & 9.07 & 3.58 & 1.28 \\
\hline HR-SC-12 & 4.54 & 1.38 & 8.19 & 9.63 & 3.41 & 1.28 \\
\hline HR-SC-14 & 4.51 & 1.41 & 7.99 & 9.60 & 3.44 & 1.34 \\
\hline HR-SC-15 & 7.90 & 1.14 & 25.2 & 13.73 & 8.46 & 1.85 \\
\hline HR-SC-16 & 6.90 & 1.38 & 19.8 & 12.18 & 8.12 & 1.58 \\
\hline HR-SC-17 & 3.49 & 1.43 & 4.71 & 7.76 & 2.06 & 1.08 \\
\hline HR-SC-18 & 4.24 & 1.42 & 7.77 & 8.97 & 3.12 & 1.24 \\
\hline HR-SC-19 & 3.76 & 0.51 & 18.5 & 8.48 & 2.35 & 1.17 \\
\hline HR-SC-20 & 4.02 & 1.40 & 6.45 & 8.94 & 2.71 & 1.13 \\
\hline HR-SC-25 & 4.15 & 1.46 & 7.26 & 8.97 & 2.95 & 1.17 \\
\hline \multicolumn{7}{|c|}{ San Gabriel study area (32 wells sampled) ${ }^{1}$} \\
\hline HR-SG-01 & 4.39 & 1.37 & 9.43 & 8.88 & 3.58 & 1.17 \\
\hline HR-SG-02 & 3.93 & 1.30 & 7.08 & 8.22 & 3.72 & 1.12 \\
\hline HR-SG-03 & 4.38 & 1.21 & 13.96 & 8.17 & 4.31 & 1.09 \\
\hline HR-SG-04 & 2.80 & 1.18 & 5.08 & 6.20 & 1.78 & 0.82 \\
\hline HR-SG-05 & 3.46 & 1.38 & 5.10 & 7.81 & 2.14 & 1.34 \\
\hline HR-SG-07 & 3.63 & 0.49 & 18.25 & 7.77 & 2.58 & 1.19 \\
\hline HR-SG-08 & 3.37 & 0.79 & 11.51 & 7.52 & 2.06 & 1.27 \\
\hline HR-SG-09 & 4.83 & 1.26 & 10.37 & 9.63 & 3.73 & 1.33 \\
\hline HR-SG-10 & 3.19 & 1.11 & 6.03 & 7.08 & 2.00 & 1.08 \\
\hline HR-SG-11 & 3.49 & 1.07 & 8.41 & 7.65 & 2.61 & 1.28 \\
\hline
\end{tabular}


Table 13. Results for analyses of dissolved noble gases by the Lawrence Livermore National Laboratory (LLNL) for samples collected for the Santa Cruz, San Gabriel, and Peninsular Ranges Hard Rock Aquifers (Hard Rock) study unit, March 2011 through March 2012, California GAMA Priority Basin Project.—Continued

[Results for noble gas analyses from the LLNL are stored in a separate database maintained by the GAMA Program. Information about the analytes given in table 3I. GAMA well identification number: HR-SC, Santa Cruz study area grid well; HR-SG, San Gabriel study area grid well; HR-SGU, San Gabriel study area understanding well; HR-PR, Peninsular Ranges study area grid well; HR-PRFP, Peninsular Ranges study area flow-path well; HR-PRU, Peninsular Ranges study area understanding well. Other abbreviations: $\mathrm{cm}^{3} \mathrm{STP} / \mathrm{g}$, cubic centimeters per gram at standard temperature and pressure]

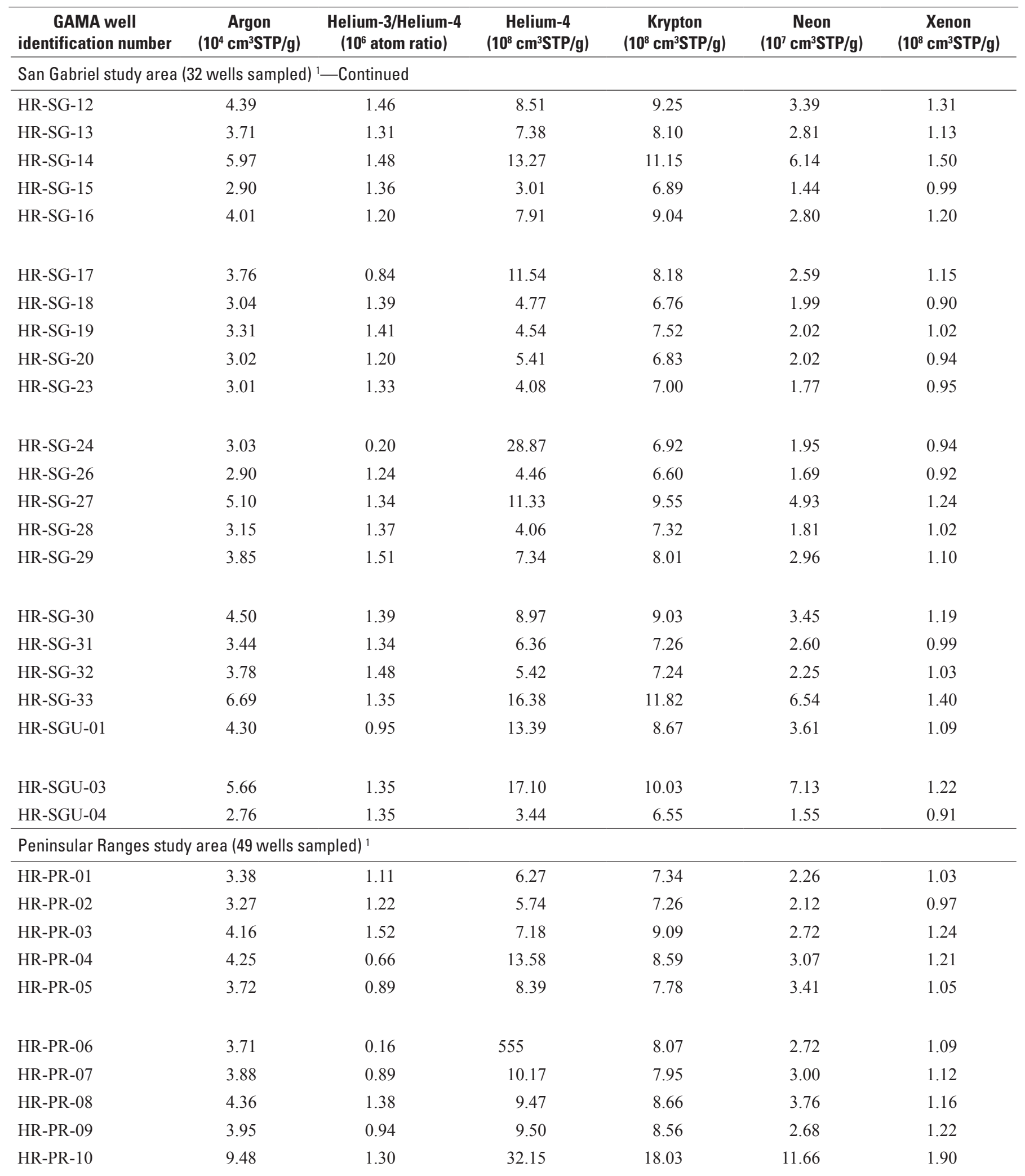


Table 13. Results for analyses of dissolved noble gases by the Lawrence Livermore National Laboratory (LLNL) for samples collected for the Santa Cruz, San Gabriel, and Peninsular Ranges Hard Rock Aquifers (Hard Rock) study unit, March 2011 through March 2012, California GAMA Priority Basin Project.-Continued

[Results for noble gas analyses from the LLNL are stored in a separate database maintained by the GAMA Program. Information about the analytes given in table 3I. GAMA well identification number: HR-SC, Santa Cruz study area grid well; HR-SG, San Gabriel study area grid well; HR-SGU, San Gabriel study area understanding well; HR-PR, Peninsular Ranges study area grid well; HR-PRFP, Peninsular Ranges study area flow-path well; HR-PRU, Peninsular Ranges study area understanding well. Other abbreviations: $\mathrm{cm}^{3} \mathrm{STP} / \mathrm{g}$, cubic centimeters per gram at standard temperature and pressure]

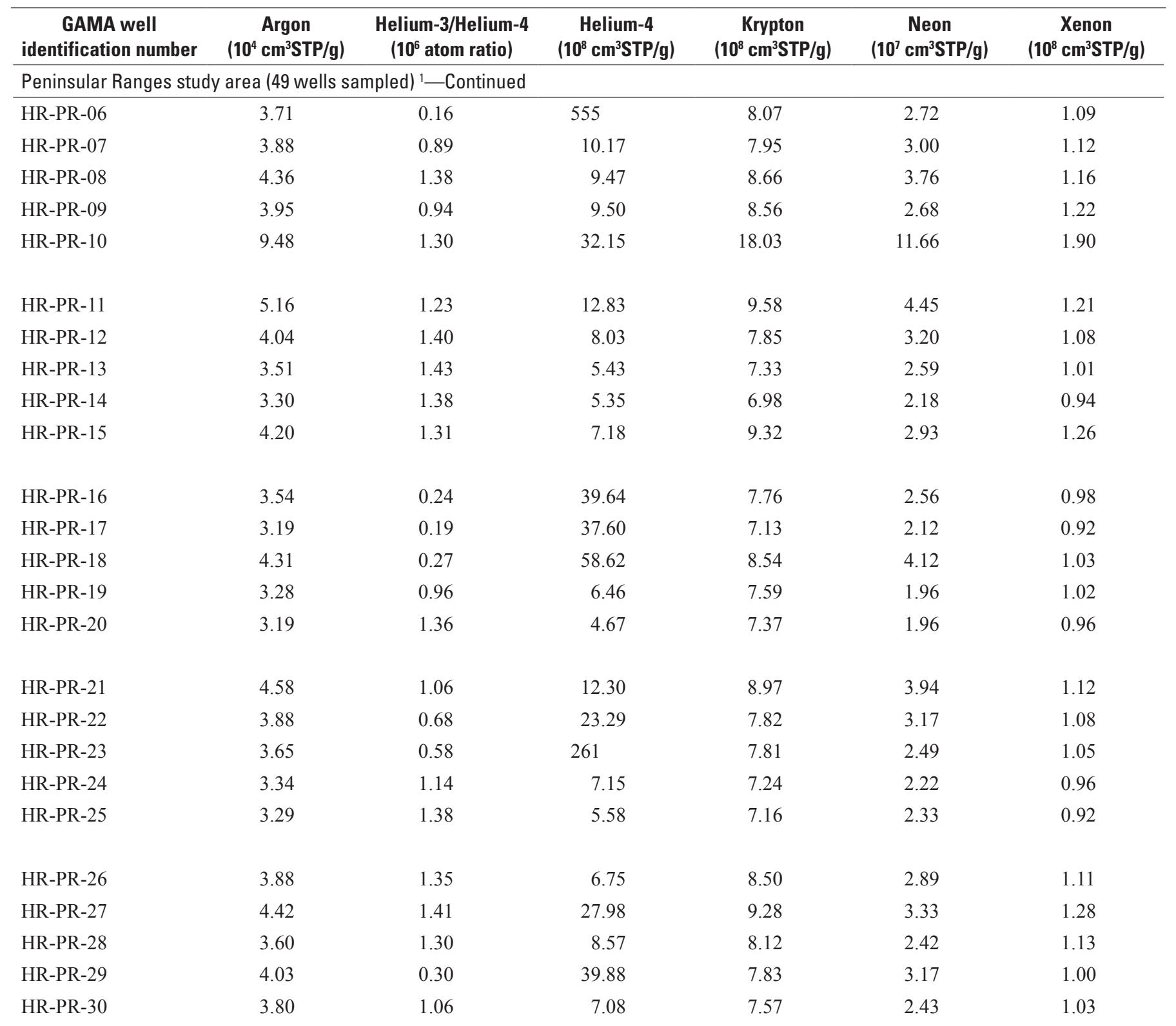


Table 13. Results for analyses of dissolved noble gases by the Lawrence Livermore National Laboratory (LLNL) for samples collected for the Santa Cruz, San Gabriel, and Peninsular Ranges Hard Rock Aquifers (Hard Rock) study unit, March 2011 through March 2012 , California GAMA Priority Basin Project.-Continued

[Results for noble gas analyses from the LLNL are stored in a separate database maintained by the GAMA Program. Information about the analytes given in table 3I. GAMA well identification number: HR-SC, Santa Cruz study area grid well; HR-SG, San Gabriel study area grid well; HR-SGU, San Gabriel study area understanding well; HR-PR, Peninsular Ranges study area grid well; HR-PRFP, Peninsular Ranges study area flow-path well; HR-PRU, Peninsular Ranges study area understanding well. Other abbreviations: $\mathrm{cm}^{3} \mathrm{STP} / \mathrm{g}$, cubic centimeters per gram at standard temperature and pressure]

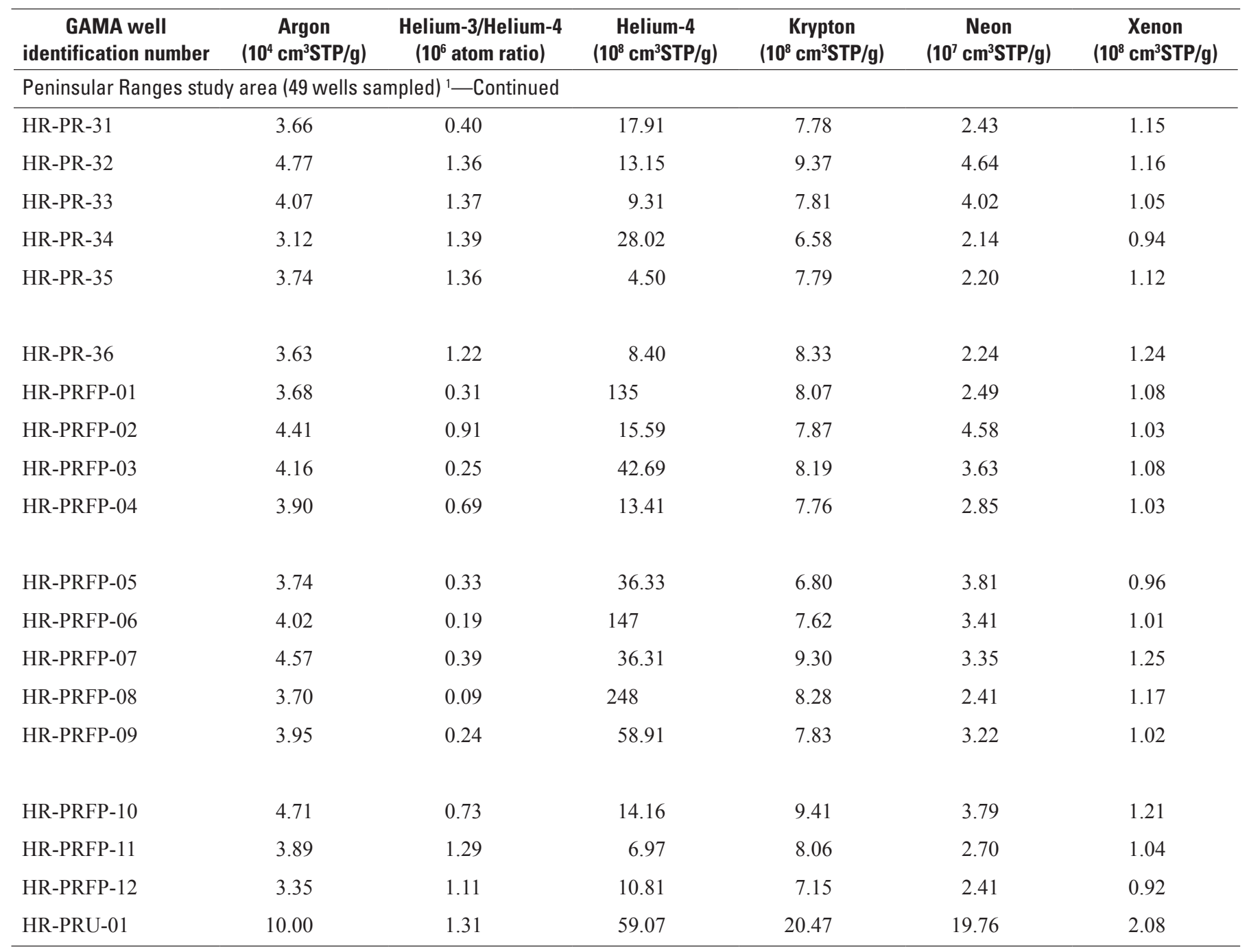

${ }^{1}$ Samples were not collected for analyses of dissolved noble gases at HR-SC-13, HR-SG-06, HR-SG-21, and HR-SGU-02, due to lack of back pressure, at HR-SC-09 and HR-SG-25 due to suspected aeration at sample point, and at HR-PRFP-13 due to short run time at the well. No result reported for HR-SG-22. 
Table 14A. Radon-222 detected in samples collected for the Santa Cruz, San Gabriel, and Peninsular Ranges Hard Rock Aquifers (Hard Rock) study unit, March 2011 through March 2012, California GAMA Priority Basin Project.

[The five-digit U.S. Geological Survey (USGS) parameter code below the constituent name is used to uniquely identify a specific constituent or property. Samples from 105 wells were analyzed. Information about the analytes are given in table $3 \mathrm{H}$. The reporting levels for radioactive constituents are given as sample-specific critical levels ( $\mathrm{ssL}_{\mathrm{c}}$ ). GAMA well identification number: HR-SC, Santa Cruz study area grid well; HR-SG, San Gabriel study area grid well; HR-SGU, San Gabriel study area understanding well; HR-PR, Peninsular Ranges study area grid well; HR-PRFP, Peninsular Ranges study area flow-path well; HR-PRU, Peninsular Ranges study area understanding well. Reporting level, benchmark type, and benchmark level as of July 6, 2012. Benchmark type: Maximum contaminant level benchmarks are listed as MCL-US when the MCL-US and MCL-CA are identical, and as MCL-CA when the MCL-CA is lower than the MCL-US or no MCL-US exists. MCL-US, U.S. Environmental Protection Agency maximum contaminant level. Other abbreviations: CSU, 1-sigma combined standard uncertainty; pCi/L, picocuries per liter; \pm , plus or minus; *, value above benchmark level]

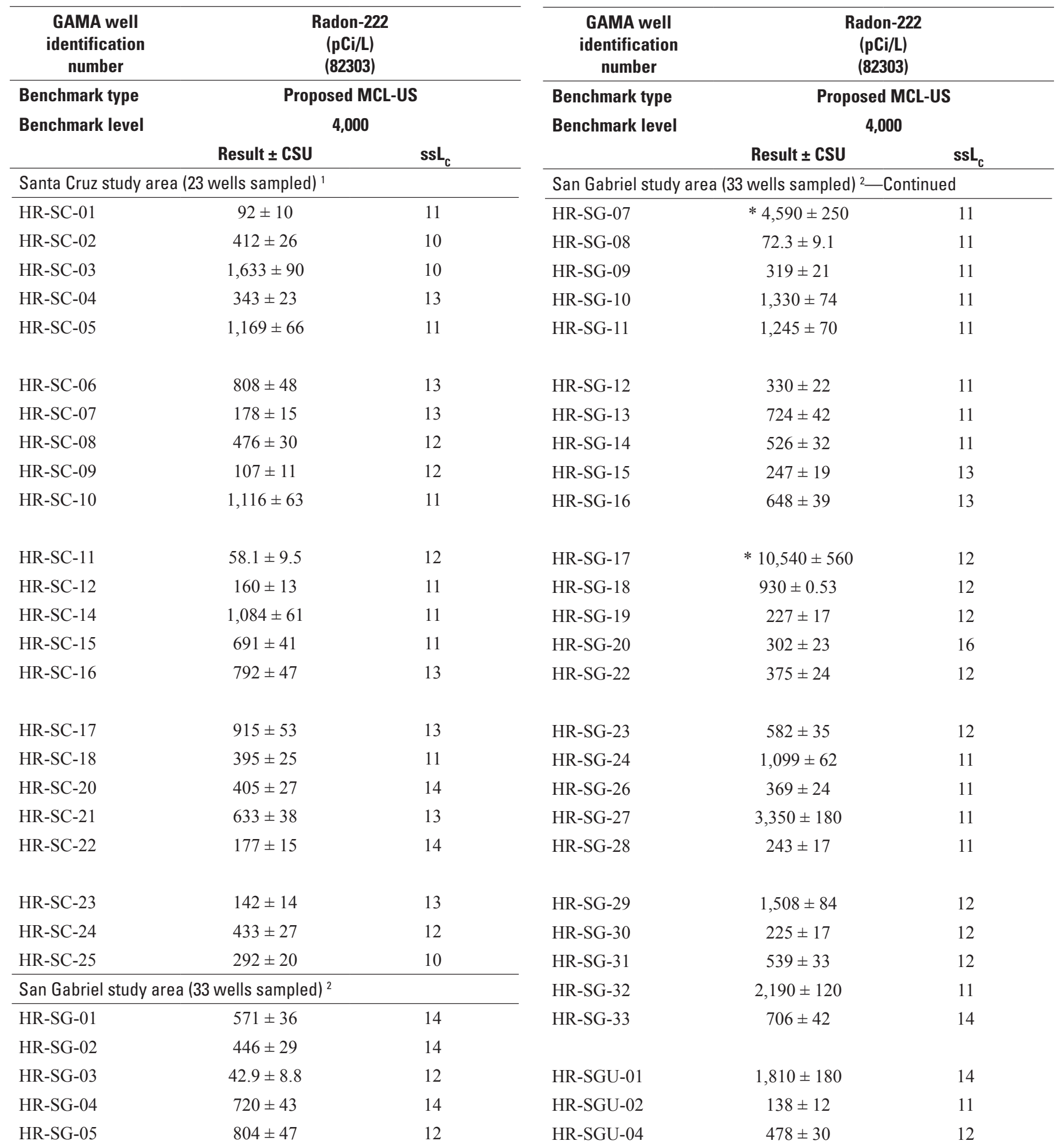


Table 14A. Radon-222 detected in samples collected for the Santa Cruz, San Gabriel, and Peninsular Ranges Hard Rock Aquifers (Hard Rock) study unit, March 2011 through March 2012, California GAMA Priority Basin Project.—Continued

[The five-digit U.S. Geological Survey (USGS) parameter code below the constituent name is used to uniquely identify a specific constituent or property. Samples from 105 wells were analyzed. Information about the analytes are given in table $3 \mathrm{H}$. The reporting levels for radioactive constituents are given as sample-specific critical levels ( $\mathrm{ssL}_{\mathrm{C}}$ ). GAMA well identification number: HR-SC, Santa Cruz study area grid well; HR-SG, San Gabriel study area grid well; HR-SGU, San Gabriel study area understanding well; HR-PR, Peninsular Ranges study area grid well; HR-PRFP, Peninsular Ranges study area flow-path well; HR-PRU, Peninsular Ranges study area understanding well. Reporting level, benchmark type, and benchmark level as of July 6, 2012. Benchmark type: Maximum contaminant level benchmarks are listed as MCL-US when the MCL-US and MCL-CA are identical, and as MCL-CA when the MCL-CA is lower than the MCL-US or no MCL-US exists. MCL-US, U.S. Environmental Protection Agency maximum contaminant level. Other abbreviations: CSU, 1-sigma combined standard uncertainty; pCi/L, picocuries per liter; \pm , plus or minus; *, value above benchmark level]

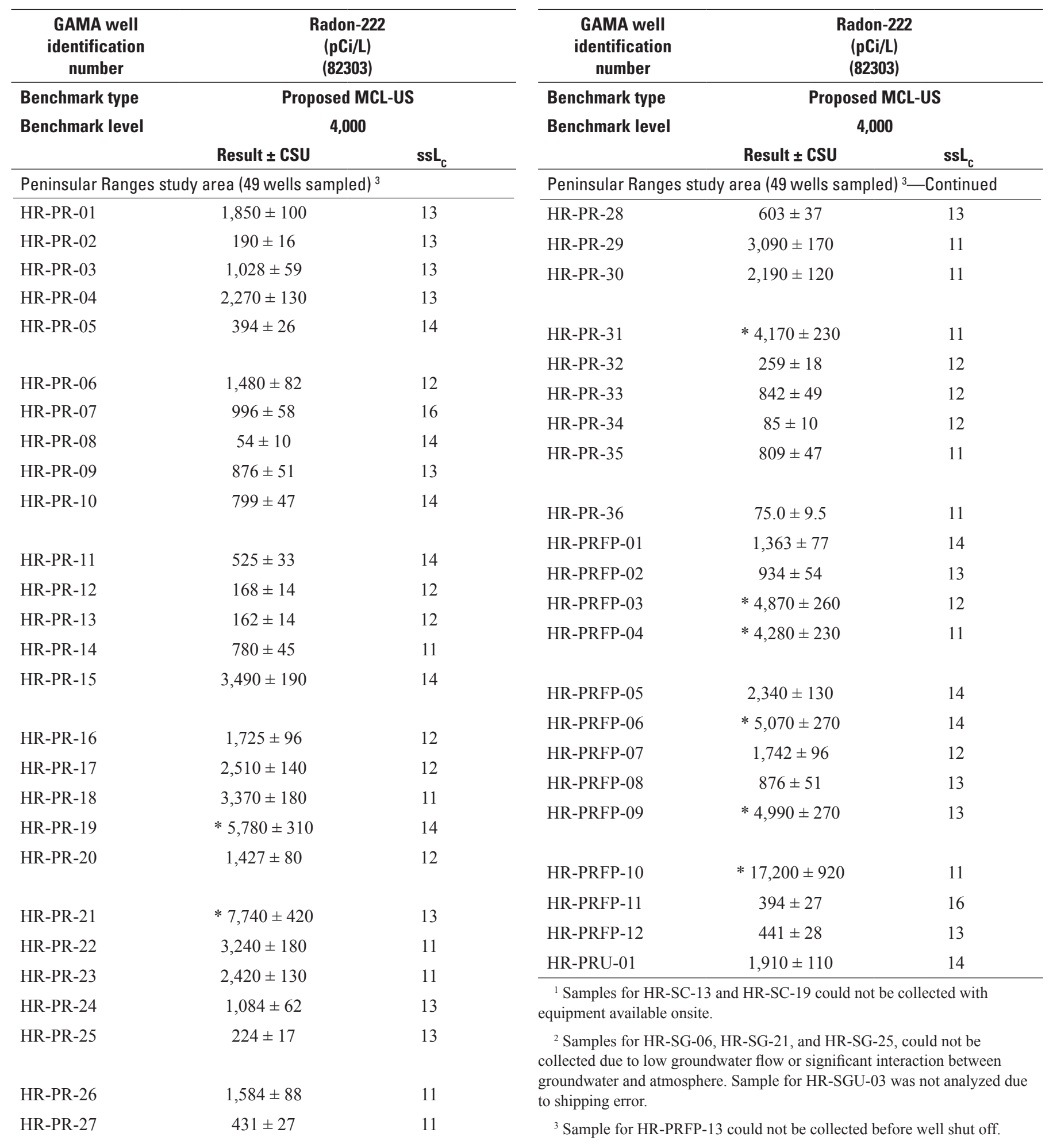


Table 14B. Gross alpha and gross beta radioactivity detected in samples collected for the Santa Cruz, San Gabriel, and Peninsular Ranges Hard Rock Aquifers (Hard Rock) study unit, March 2011 through March 2012, California GAMA Priority Basin Project.

[The five-digit U.S. Geological Survey (USGS) parameter code below the constituent name is used to uniquely identify a specific constituent or property. Samples from 110 wells were analyzed. Information about the analytes given in table $3 \mathrm{H}$. The reference nuclide for measurement of gross alpha radioactivity is thorium-230, and the reference nuclide for measurement of gross beta radioactivity is cesium-137. The reporting levels for radioactive constituents are given as sample-specific critical levels $\left(\mathrm{ssL}_{\mathrm{C}}\right)$. Measured values less than the $\mathrm{ssL}_{\mathrm{C}}$ are reported as not detected $(-)$. GAMA well identification number: HR-SC, Santa Cruz study area grid well; HR-SG, San Gabriel study area grid well; HR-SGU, San Gabriel study area understanding well; HR-PR, Peninsular Ranges study area grid well; HR-PRFP, Peninsular Ranges study area flow-path well; HR-PRU, Peninsular Ranges study area understanding well. Reporting levels, benchmark types, and benchmark levels as of July 6, 2012. Benchmark type: Maximum contaminant level benchmarks are listed as MCL-US when the MCL-US and MCL-CA are identical, and as MCL-CA when the MCL-CA is lower than the MCL-US or no MCL-US exists. MCL-US, U.S. Environmental Protection Agency maximum contaminant level; MCL-CA, California Department of Public Health (CDPH) maximum contaminant level. Other abbreviations: CSU, 1-sigma combined standard uncertainty; $\mathrm{pCi} / \mathrm{L}$, picocuries per liter; \pm , plus or minus; na, not available; *, value above benchmark level]

\begin{tabular}{|c|c|c|c|c|c|c|c|c|}
\hline $\begin{array}{c}\text { GAMA } \\
\text { well } \\
\text { identification } \\
\text { number }\end{array}$ & \multicolumn{2}{|c|}{$\begin{array}{c}\text { Gross alpha radioactivity, } \\
\text { 72-hour count } \\
\text { (pCi/L) } \\
(62636)\end{array}$} & \multicolumn{2}{|c|}{$\begin{array}{c}\text { Gross alpha radioactivity, } \\
\text { 30-day count } \\
\text { (pCi/L) } \\
(62639)\end{array}$} & \multicolumn{2}{|c|}{$\begin{array}{c}\text { Gross beta radioactivity, } \\
\text { 72-hour count } \\
\text { (pCi/L) } \\
(62642)\end{array}$} & \multicolumn{2}{|c|}{$\begin{array}{c}\text { Gross beta radioactivity, } \\
\text { 30-day count } \\
\text { (pCi/L) } \\
(62645)\end{array}$} \\
\hline \multirow[t]{2}{*}{ Benchmark level } & \multicolumn{2}{|c|}{15} & \multicolumn{2}{|c|}{15} & \multicolumn{2}{|c|}{50} & \multicolumn{2}{|c|}{50} \\
\hline & Result \pm CSU & $\operatorname{ssL}_{c}$ & Result \pm CSU & ssL $_{c}$ & Result \pm CSU & $\mathrm{ssL}_{\mathrm{c}}$ & Result \pm CSU & $\operatorname{ssL}_{c}$ \\
\hline HR-SC-01 & - & 0.27 & - & 0.48 & - & 0.47 & $0.5 \pm 0.3$ & 0.47 \\
\hline HR-SC-02 & $0.8 \pm 0.4$ & 0.46 & - & 0.51 & $2.64 \pm 0.43$ & 0.61 & $1.95 \pm 0.38$ & 0.53 \\
\hline HR-SC-03 & - & 1.1 & $2.55 \pm 0.84$ & 0.88 & $2.54 \pm 0.38$ & 0.53 & $2.4 \pm 0.35$ & 0.47 \\
\hline HR-SC-04 & $2.17 \pm 0.93^{2}$ & 1.1 & $2.1 \pm 0.97$ & 1.1 & $2.5 \pm 0.39$ & 0.52 & $2.66 \pm 0.39$ & 0.52 \\
\hline HR-SC-07 & $4.5 \pm 1.6$ & 1.7 & - & 2 & $4.3 \pm 0.6$ & 0.74 & $3.99 \pm 0.73$ & 1 \\
\hline HR-SC-08 & $3 \pm 0.63$ & 0.5 & $0.70 \pm 0.37$ & 0.45 & $2.08 \pm 0.34$ & 0.46 & $1.45 \pm 0.34$ & 0.49 \\
\hline HR-SC-09 & - & 1.3 & -3 & 1.8 & $1.04 \pm 0.36$ & 0.56 & $1.3 \pm 0.4^{4}$ & 0.6 \\
\hline HR-SC-10 & - & 0.4 & - & 0.36 & - & 0.43 & - & 0.45 \\
\hline HR-SC-11 & $2.47 \pm 0.65$ & 0.49 & - & 0.97 & $1.16 \pm 0.48$ & 0.74 & $0.9 \pm 0.5$ & 0.84 \\
\hline HR-SC-12 & - & 0.75 & - & 0.89 & - & 0.83 & - & 0.91 \\
\hline HR-SC-13 & - & 0.38 & - & 0.4 & - & 0.47 & - & 0.49 \\
\hline HR-SC-14 & $1.24 \pm 0.45$ & 0.46 & $1.16 \pm 0.47$ & 0.5 & $1.65 \pm 0.48$ & 0.73 & $1.71 \pm 0.56$ & 0.85 \\
\hline HR-SC-19 & $2.2 \pm 0.78$ & 0.9 & - & 0.77 & $4.5 \pm 0.6$ & 0.78 & $4.51 \pm 0.84$ & 1.2 \\
\hline HR-SC-20 & - & 1.9 & - & 2.1 & $0.95 \pm 0.55$ & 0.84 & $0.84 \pm 0.42$ & 0.66 \\
\hline HR-SC-21 & - & 1.6 & - & 1.5 & $11.9 \pm 0.86$ & 0.62 & $9.92 \pm 0.74$ & 0.62 \\
\hline HR-SC-22 & $-^{2,3}$ & 1.5 & -3 & 1.5 & $1.69 \pm 0.37^{2}$ & 0.54 & $1.48 \pm 0.46$ & 0.69 \\
\hline HR-SC-23 & -2 & 1.2 & - & 1.3 & $4.36 \pm 0.48^{2}$ & 0.58 & $4.1 \pm 0.42$ & 0.48 \\
\hline HR-SC-24 & $6.2 \pm 2.1$ & 2.4 & - & 2.3 & $2.33 \pm 0.66$ & 0.97 & $3.55 \pm 0.58$ & 0.8 \\
\hline HR-SC-25 & $1.25 \pm 0.31$ & 0.25 & - & 0.36 & $0.89 \pm 0.28$ & 0.42 & $0.54 \pm 0.27$ & 0.42 \\
\hline
\end{tabular}


Table 14B. Gross alpha and gross beta radioactivity detected in samples collected for the Santa Cruz, San Gabriel, and Peninsular Ranges Hard Rock Aquifers (Hard Rock) study unit, March 2011 through March 2012, California GAMA Priority Basin Project.Continued

[The five-digit U.S. Geological Survey (USGS) parameter code below the constituent name is used to uniquely identify a specific constituent or property. Samples from 110 wells were analyzed. Information about the analytes given in table $3 \mathrm{H}$. The reference nuclide for measurement of gross alpha radioactivity is thorium-230, and the reference nuclide for measurement of gross beta radioactivity is cesium-137. The reporting levels for radioactive constituents are given as sample-specific critical levels $\left(\mathrm{ssL}_{\mathrm{C}}\right)$. Measured values less than the $\mathrm{ssL}_{\mathrm{C}}$ are reported as not detected (一). GAMA well identification number: HR-SC, Santa Cruz study area grid well; HR-SG, San Gabriel study area grid well; HR-SGU, San Gabriel study area understanding well; HR-PR, Peninsular Ranges study area grid well; HR-PRFP, Peninsular Ranges study area flow-path well; HR-PRU, Peninsular Ranges study area understanding well. Reporting levels, benchmark types, and benchmark levels as of July 6, 2012. Benchmark type: Maximum contaminant level benchmarks are listed as MCL-US when the MCL-US and MCL-CA are identical, and as MCL-CA when the MCL-CA is lower than the MCL-US or no MCL-US exists. MCL-US, U.S. Environmental Protection Agency maximum contaminant level; MCL-CA, California Department of Public Health (CDPH) maximum contaminant level. Other abbreviations: CSU, 1-sigma combined standard uncertainty; $\mathrm{pCi} / \mathrm{L}$, picocuries per liter; \pm , plus or minus; na, not available; *, value above benchmark level]

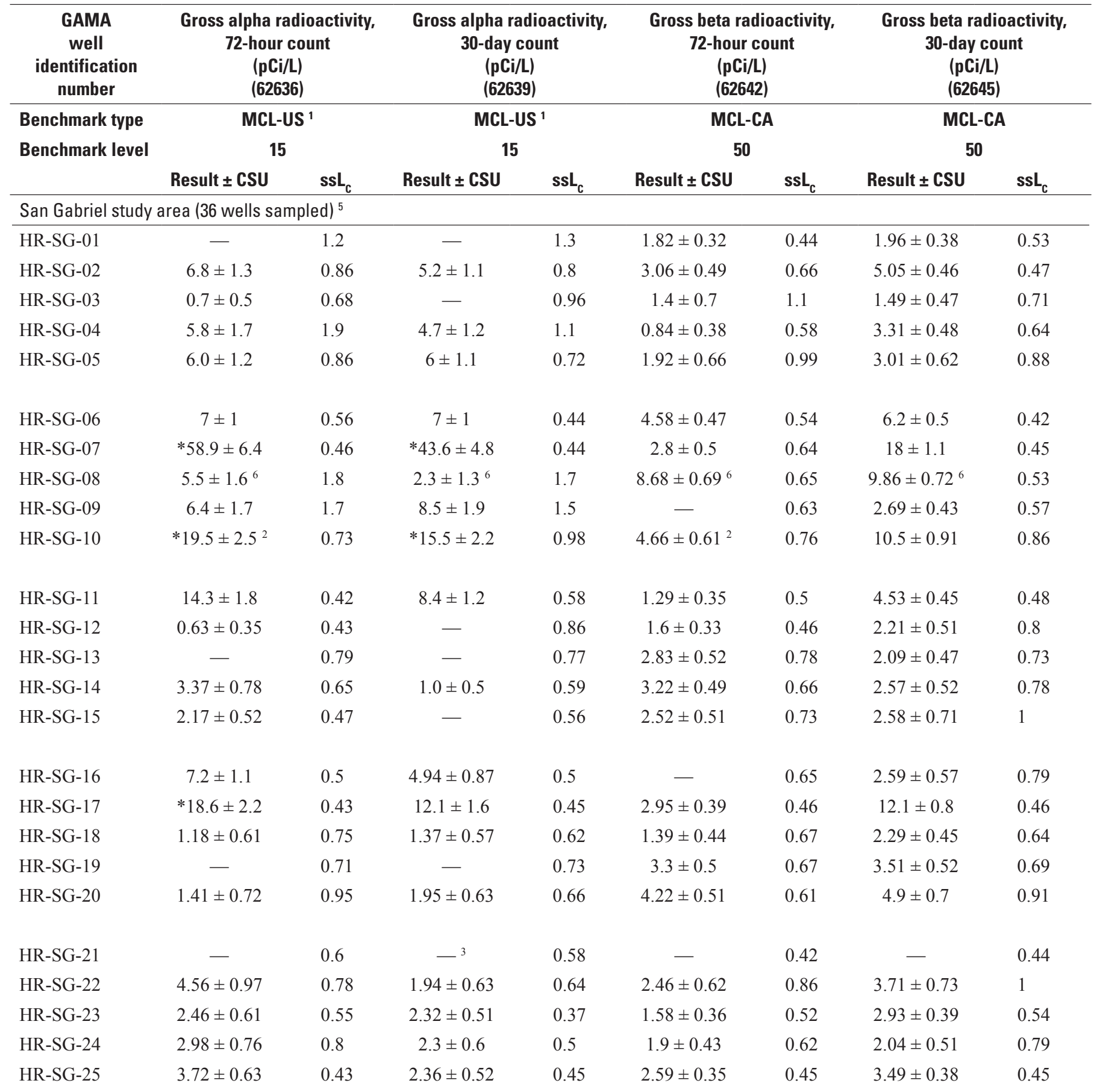


Table 14B. Gross alpha and gross beta radioactivity detected in samples collected for the Santa Cruz, San Gabriel, and Peninsular Ranges Hard Rock Aquifers (Hard Rock) study unit, March 2011 through March 2012, California GAMA Priority Basin Project.Continued

[The five-digit U.S. Geological Survey (USGS) parameter code below the constituent name is used to uniquely identify a specific constituent or property. Samples from 110 wells were analyzed. Information about the analytes given in table $3 \mathrm{H}$. The reference nuclide for measurement of gross alpha radioactivity is thorium-230, and the reference nuclide for measurement of gross beta radioactivity is cesium-137. The reporting levels for radioactive constituents are given as sample-specific critical levels $\left(\mathrm{ssL}_{\mathrm{C}}\right)$. Measured values less than the $\mathrm{ssL}_{\mathrm{C}}$ are reported as not detected $(-)$. GAMA well identification number: HR-SC, Santa Cruz study area grid well; HR-SG, San Gabriel study area grid well; HR-SGU, San Gabriel study area understanding well; HR-PR, Peninsular Ranges study area grid well; HR-PRFP, Peninsular Ranges study area flow-path well; HR-PRU, Peninsular Ranges study area understanding well. Reporting levels, benchmark types, and benchmark levels as of July 6, 2012. Benchmark type: Maximum contaminant level benchmarks are listed as MCL-US when the MCL-US and MCL-CA are identical, and as MCL-CA when the MCL-CA is lower than the MCL-US or no MCL-US exists. MCL-US, U.S. Environmental Protection Agency maximum contaminant level; MCL-CA, California Department of Public Health (CDPH) maximum contaminant level. Other abbreviations: CSU, 1-sigma combined standard uncertainty; $\mathrm{pCi} / \mathrm{L}$, picocuries per liter; \pm , plus or minus; na, not available; *, value above benchmark level]

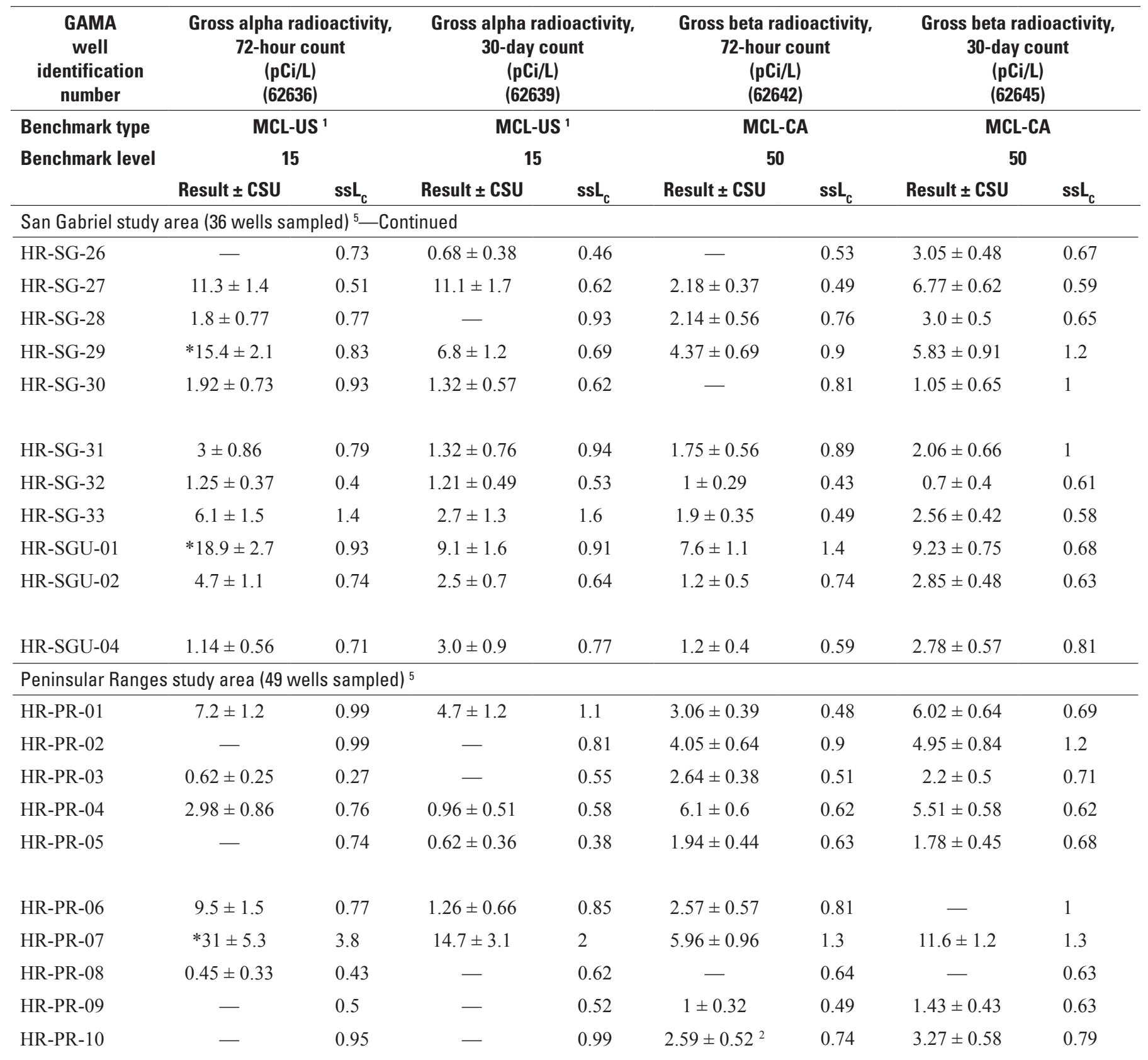


Table 14B. Gross alpha and gross beta radioactivity detected in samples collected for the Santa Cruz, San Gabriel, and Peninsular Ranges Hard Rock Aquifers (Hard Rock) study unit, March 2011 through March 2012, California GAMA Priority Basin Project.Continued

[The five-digit U.S. Geological Survey (USGS) parameter code below the constituent name is used to uniquely identify a specific constituent or property. Samples from 110 wells were analyzed. Information about the analytes given in table $3 \mathrm{H}$. The reference nuclide for measurement of gross alpha radioactivity is thorium-230, and the reference nuclide for measurement of gross beta radioactivity is cesium-137. The reporting levels for radioactive constituents are given as sample-specific critical levels $\left(\mathrm{ssL}_{\mathrm{C}}\right)$. Measured values less than the $\mathrm{ssL}_{\mathrm{C}}$ are reported as not detected (-). GAMA well identification number: HR-SC, Santa Cruz study area grid well; HR-SG, San Gabriel study area grid well; HR-SGU, San Gabriel study area understanding well; HR-PR, Peninsular Ranges study area grid well; HR-PRFP, Peninsular Ranges study area flow-path well; HR-PRU, Peninsular Ranges study area understanding well. Reporting levels, benchmark types, and benchmark levels as of July 6, 2012. Benchmark type: Maximum contaminant level benchmarks are listed as MCL-US when the MCL-US and MCL-CA are identical, and as MCL-CA when the MCL-CA is lower than the MCL-US or no MCL-US exists. MCL-US, U.S. Environmental Protection Agency maximum contaminant level; MCL-CA, California Department of Public Health (CDPH) maximum contaminant level. Other abbreviations: CSU, 1-sigma combined standard uncertainty; $\mathrm{pCi} / \mathrm{L}$, picocuries per liter; \pm , plus or minus; na, not available; *, value above benchmark level]

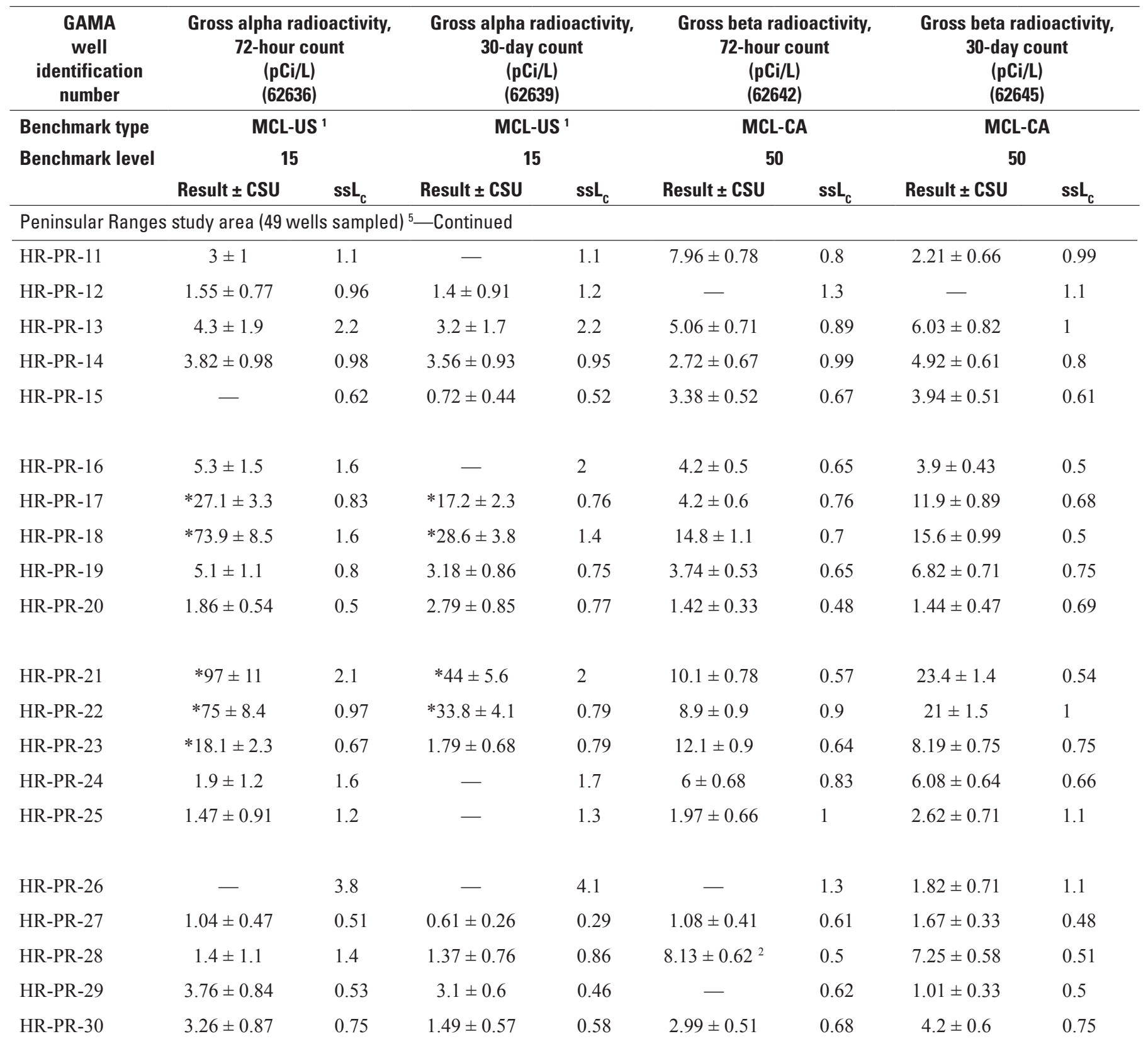


Table 14B. Gross alpha and gross beta radioactivity detected in samples collected for the Santa Cruz, San Gabriel, and Peninsular Ranges Hard Rock Aquifers (Hard Rock) study unit, March 2011 through March 2012, California GAMA Priority Basin Project.Continued

[The five-digit U.S. Geological Survey (USGS) parameter code below the constituent name is used to uniquely identify a specific constituent or property. Samples from 110 wells were analyzed. Information about the analytes given in table $3 \mathrm{H}$. The reference nuclide for measurement of gross alpha radioactivity is thorium-230, and the reference nuclide for measurement of gross beta radioactivity is cesium-137. The reporting levels for radioactive constituents are given as sample-specific critical levels $\left(\mathrm{ssL}_{\mathrm{C}}\right)$. Measured values less than the $\mathrm{ssL}_{\mathrm{C}}$ are reported as not detected $(-)$. GAMA well identification number: HR-SC, Santa Cruz study area grid well; HR-SG, San Gabriel study area grid well; HR-SGU, San Gabriel study area understanding well; HR-PR, Peninsular Ranges study area grid well; HR-PRFP, Peninsular Ranges study area flow-path well; HR-PRU, Peninsular Ranges study area understanding well. Reporting levels, benchmark types, and benchmark levels as of July 6, 2012. Benchmark type: Maximum contaminant level benchmarks are listed as MCL-US when the MCL-US and MCL-CA are identical, and as MCL-CA when the MCL-CA is lower than the MCL-US or no MCL-US exists. MCL-US, U.S. Environmental Protection Agency maximum contaminant level; MCL-CA, California Department of Public Health (CDPH) maximum contaminant level. Other abbreviations: CSU, 1-sigma combined standard uncertainty; $\mathrm{pCi} / \mathrm{L}$, picocuries per liter; \pm , plus or minus; na, not available; *, value above benchmark level]

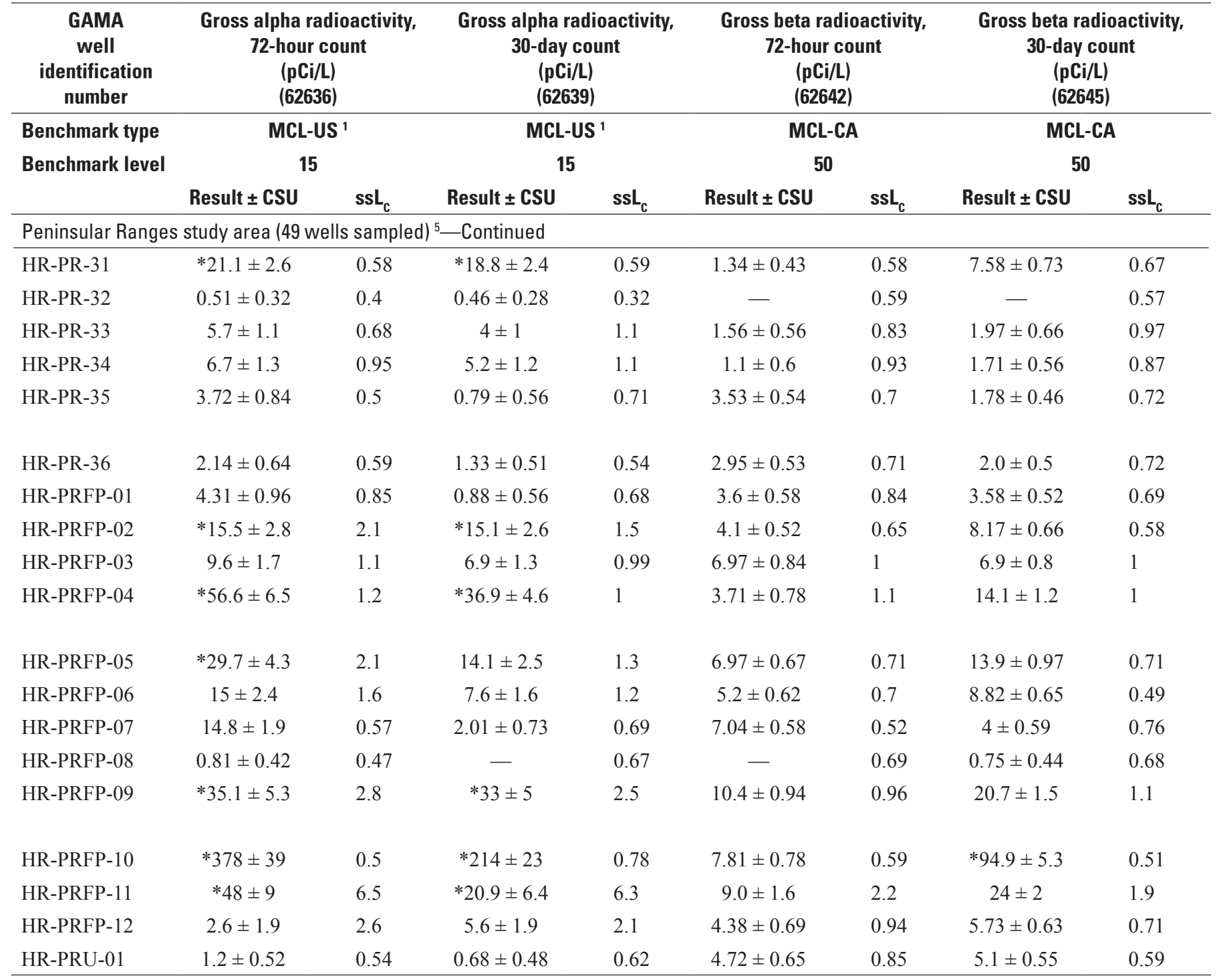

${ }^{1}$ The MCL-US applies to adjusted gross alpha radioactivity, which is equal to gross alpha activity minus uranium activity. Results were not adjusted for uranium activity.

${ }^{2}$ Gross alpha activity (72-hour) holding time exceeded by 4 days from time of sample collection for HR-SC-04 and HR-SG-10; gross beta activity (72-hour) holding time exceeded by 4 days from time of sample collection for HR-SG-10, HR-PR-10, and HR-PR-28. Gross alpha and beta activities (72-hour) holding times exceeded by 8 days from time of sample collection for HR-SC-22 and HR-SC-23.

${ }^{3}$ Laboratory comment: negative result may indicate potential negative bias.

${ }^{4}$ Activity for HR-SC-09 was counted 18 days after 72-hour count rather than 30 days.

${ }^{5}$ Samples for HR-SGU-03 and HR-PRFP-13 were not analyzed due to shipping error.

${ }^{6}$ Variability for the HR-SG-08 replicate pair was outside the acceptable limits for all gross alpha and beta radioactivity results. The replicate sample results were generally lower than the environmental sample results. See table A4C for results for the replicate pair. 
Table 14C. Uranium activities detected in samples collected for the Santa Cruz, San Gabriel, and Peninsular Ranges Hard Rock Aquifers (Hard Rock) study unit, March 2011 through March 2012, California GAMA Priority Basin Project.

[The five-digit U.S. Geological Survey (USGS) parameter code below the constituent name is used to uniquely identify a specific constituent or property. Samples from 29 wells were analyzed. Information about the analytes given in table $3 \mathrm{H}$. The reporting levels for radioactive constituents are given as samplespecific critical levels $\left(\mathrm{ssL}_{\mathrm{C}}\right)$. Measured values less than the $\mathrm{ssL}_{\mathrm{C}}$ are reported as not detected ( - ). Only samples with total uranium concentrations (table 8 ) greater than $10 \mu \mathrm{g} / \mathrm{L}$ were analyzed for isotopic composition. GAMA well identification number: HR-SC, Santa Cruz study area grid well; HR-SG, San Gabriel study area grid well; HR-SGU, San Gabriel study area understanding well; HR-PR, Peninsular Ranges study area grid well; HR-PRFP, Peninsular Ranges study area flow-path well; HR-PRU, Peninsular Ranges study area understanding well. Reporting levels, benchmark types, and benchmark levels as of July 6, 2012. Benchmark type: Maximum contaminant level benchmarks are listed as MCL-US when the MCL-US and MCL-CA are identical, and as MCL-CA when the MCL-CA is lower than the MCL-US or no MCL-US exists. MCL-US, U.S. Environmental Protection Agency maximum contaminant level; MCL-CA, California Department of Public Health (CDPH) maximum contaminant level. Other abbreviations: CSU, 1-sigma combined standard uncertainty; $\mathrm{pCi} / \mathrm{L}$, picocuries per liter; \pm , plus or minus; *, the sum of uranium-234, -235 , and -238 concentrations is greater than the benchmark level]

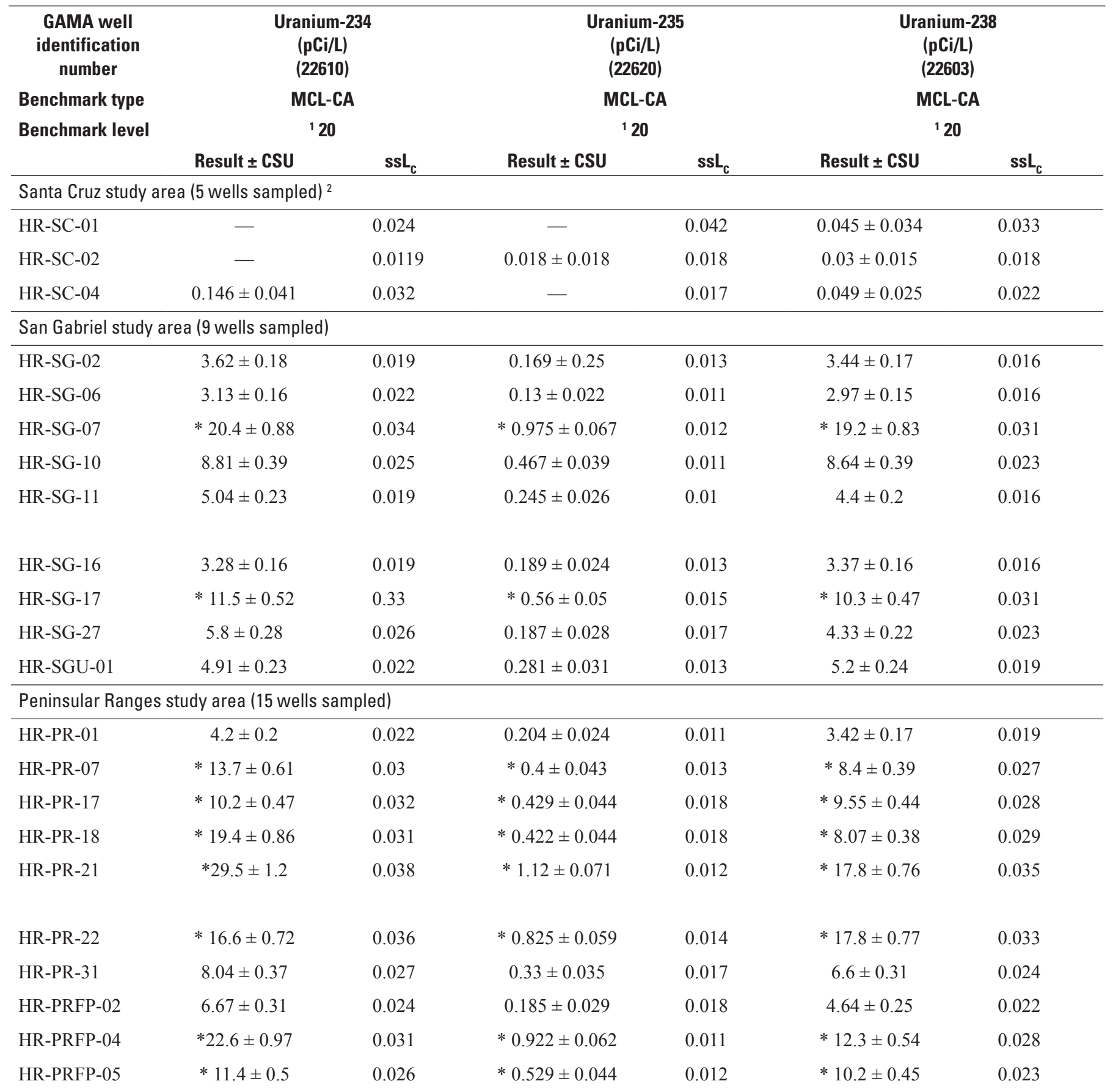


Table 14C. Uranium activities detected in samples collected for the Santa Cruz, San Gabriel, and Peninsular Ranges Hard Rock Aquifers (Hard Rock) study unit, March 2011 through March 2012, California GAMA Priority Basin Project.—Continued

[The five-digit U.S. Geological Survey (USGS) parameter code below the constituent name is used to uniquely identify a specific constituent or property. Samples from 29 wells were analyzed. Information about the analytes given in table $3 \mathrm{H}$. The reporting levels for radioactive constituents are given as samplespecific critical levels $\left(\mathrm{ssL}_{\mathrm{C}}\right)$. Measured values less than the $\mathrm{ssL}_{\mathrm{C}}$ are reported as not detected (-). Only samples with total uranium concentrations (table 8 ) greater than $10 \mu \mathrm{g} / \mathrm{L}$ were analyzed for isotopic composition. GAMA well identification number: HR-SC, Santa Cruz study area grid well; HR-SG, San Gabriel study area grid well; HR-SGU, San Gabriel study area understanding well; HR-PR, Peninsular Ranges study area grid well; HR-PRFP, Peninsular Ranges study area flow-path well; HR-PRU, Peninsular Ranges study area understanding well. Reporting levels, benchmark types, and benchmark levels as of July 6, 2012. Benchmark type: Maximum contaminant level benchmarks are listed as MCL-US when the MCL-US and MCL-CA are identical, and as MCL-CA when the MCL-CA is lower than the MCL-US or no MCL-US exists. MCL-US, U.S. Environmental Protection Agency maximum contaminant level; MCL-CA, California Department of Public Health (CDPH) maximum contaminant level. Other abbreviations: CSU, 1-sigma combined standard uncertainty; $\mathrm{pCi} / \mathrm{L}$, picocuries per liter; \pm , plus or minus; *, the sum of uranium-234, -235 , and -238 concentrations is greater than the benchmark level]

\begin{tabular}{|c|c|c|c|c|c|c|}
\hline $\begin{array}{c}\text { GAMA well } \\
\text { identification } \\
\text { number }\end{array}$ & \multicolumn{2}{|c|}{$\begin{array}{c}\text { Uranium-234 } \\
\text { (pCi/L) } \\
(22610)\end{array}$} & \multicolumn{2}{|c|}{$\begin{array}{c}\text { Uranium-235 } \\
\text { (pCi/L) } \\
(22620)\end{array}$} & \multicolumn{2}{|c|}{$\begin{array}{c}\text { Uranium-238 } \\
\text { (pCi/L) } \\
(22603)\end{array}$} \\
\hline Benchmark type & \multicolumn{2}{|c|}{ MCL-CA } & \multicolumn{2}{|c|}{ MCL-CA } & \multicolumn{2}{|c|}{ MCL-CA } \\
\hline \multirow[t]{2}{*}{ Benchmark level } & \multicolumn{2}{|c|}{${ }^{1} 20$} & \multicolumn{2}{|c|}{${ }^{1} 20$} & \multicolumn{2}{|c|}{${ }^{1} 20$} \\
\hline & Result \pm CSU & $\mathbf{s s L}_{\mathrm{c}}$ & Result \pm CSU & $\mathbf{s s L}_{\mathrm{c}}$ & Result \pm CSU & sst $_{c}$ \\
\hline \multicolumn{7}{|c|}{ Peninsular Ranges study area ( 15 wells sampled)_Continued } \\
\hline HR-PRFP-09 & $* 19.7 \pm 0.84$ & 0.026 & $* 0.59 \pm 0.046$ & 0.011 & $* 13.8 \pm 0.6$ & 0.024 \\
\hline HR-PRFP-10 & $* 40.4 \pm 1.7$ & 0.035 & $* 1.35 \pm 0.077$ & 0.01 & $* 28.2 \pm 1.2$ & 0.033 \\
\hline HR-PRFP-11 & $* 26.7 \pm 1.1$ & 0.033 & $* 1.06 \pm 0.07$ & 0.0098 & $* 21.2 \pm 0.91$ & 0.03 \\
\hline HR-PRFP-13 & $* 19.8 \pm 0.85$ & 0.029 & $* 0.716 \pm 0.054$ & 0.012 & $* 15.6 \pm 0.68$ & 0.027 \\
\hline
\end{tabular}

${ }^{1}$ The MCL-CA benchmark for uranium is the sum of uranium-234, -235 , and -238 .

${ }^{2}$ All 25 Santa Cruz study area wells had samples with total uranium concentrations less than 10 micrograms per liter (see table 8 ). Samples HR-SC-01 through HR-SC-05 were requested for analysis of uranium isotopic composition by error. HR-SC-01, HR-SC-02, and HR-SC-04 had very low-level uranium activities, but are listed in this table for reporting purposes. HR-SC-03 and HR-SC-05 had no detectable uranium activities. 


\section{Appendix}

This appendix includes discussions of the methods used to collect and analyze groundwater samples and report the resulting water-quality data. These methods were selected to obtain representative samples of the groundwater from each well and to minimize the potential for contamination of the samples or bias in the data. Procedures used to collect and assess QC data and the results of the QC assessments also are discussed.

In the Hard Rock study unit, groundwater samples were collected and QA procedures were implemented by using standard and modified USGS protocols from the NAWQA Program (Koterba and others, 1995), the NFM (U.S. Geological Survey, variously dated), and protocols described by Shelton and others (2001) and Wright and others (2005). The QA plan followed by the NWQL, the primary laboratory used to analyze samples for this study, is described by Stevenson (2013).

\section{Sample Collection and Analysis}

Samples were collected from two types of sites: production wells and springs ("springs" in this study unit include horizontal wells). Sites classified as production wells are vertically drilled into the ground and have pumps that bring the groundwater from the aquifer to a distribution system. Sites were classified as springs if groundwater could discharge from the aquifer into the distribution system without a pump and if the well was either drilled horizontally or had no drilled hole. A few springs had pumps to transport groundwater from the spring to a storage tank at a higher elevation.

In most cases, wells were pumped continuously to purge at least three casing volumes of water from the well prior to sampling (Wilde, 2006a). In some cases, continuous pumping was limited to 2 hours, either because of limited space in storage tanks for the pumped water or because of drawdown of the water table; in these cases, a minimum of one casing volume of groundwater was pumped from each well. The limitation on pumping did not allow sufficient time to complete the sampling of at least one well, and samples for analysis of some constituent groups were not collected from these wells (tables 13, 14A). For all sites, field measurements of dissolved oxygen, water temperature, $\mathrm{pH}$, and specific conductance were recorded at 3 to 5 minute intervals before sampling and then checked against the stabilization criteria described in the NFM to ensure purging was sufficient (Wilde, 2008).

Wells were sampled by using Teflon ${ }^{\circledR}$ tubing with brass and stainless-steel fittings attached to a sampling point (usually a hose-bib fitting) on the well discharge pipe as close to the wellhead as possible. The sampling point was located upstream from water-storage tanks and from the wellhead treatment system (if present). All samples were collected outdoors by connecting a 1 - to 3 -foot length of Teflon ${ }^{\circledR}$ tubing to the sampling point (Lane and others, 2003). All fittings and lengths of tubing were cleaned between samples (Wilde, 2004).

If a chlorinating system was attached to the well, the chlorinator was shut off, when possible, before the well was purged and sampled, in order to clear all chlorine out of the system. The absence of free chlorine was verified by using a $\mathrm{Hach}^{\circledR}$ field test kit. In one well, the chlorinator was not shut off prior to sampling. In this case, samples for analysis of organic constituents (VOCs and pesticides) were collected in amber bottles containing ascorbic acid and buffer (Wilde, 2009).

For the field measurements, groundwater was pumped through a flow-through chamber that was attached to the sampling point and fitted with a YSI ${ }^{\circledR}$ multi-probe meter that simultaneously measured the field water-quality indicatorsdissolved oxygen, temperature, $\mathrm{pH}$, and specific conductance. Field measurements were made in accordance with protocols in the USGS NFM (Radtke and others, 2005; Lewis, 2006; Wilde, 2006b, 2008; Ritz and Collins, 2008). All sensors on the multi-probe meter were calibrated daily. Measured dissolved oxygen, temperature, $\mathrm{pH}$, and specific conductance values were recorded at 5-minute ( $\mathrm{min}$ ) intervals, and when these values remained stable for a minimum of $30 \mathrm{~min}$, samples for laboratory analyses then were collected. If runtime was limited but the well was used often, then at least five values were recorded at 3-min intervals.

Most of the springs were plumbed and had sampling points on the discharge pipes similar to the sampling points found on discharge pipes from wells. Field water-quality indicators were measured and samples were collected by using the same protocols as used for wells.

A few springs were not plumbed and discharged into pools, from which the water was then pumped into the distribution system. For these springs, field water-quality parameters were measured by dipping the multi-probe meter directly into the water as close to the spring discharge point as possible. Groundwater samples were collected by dipping bottles into the water as close to the spring discharge point as possible. Turbidity was measured in the mobile laboratory with a calibrated turbidity meter (Anderson, 2005). For all samples collected from springs with pools or low pressure, turbidity was less than 0.5 nephelometric turbidity unit (NTU); the samples were collected unfiltered, but were marked as filtered for analysis. This method was used because the alternative - pumping water from a pool with a portable peristaltic pump through the filtration apparatus - was not logistically possible at most of the unplumbed spring sites. 
Field measurements and instrument calibrations were recorded by hand on field record sheets and electronically in the USGS Personal Computer Field Form (PCFF) program. Analytical service requests for the NWQL were generated by PCFF, whereas analytical service requests for non-NWQL analysis were entered into laboratory-specific spreadsheets. Information from PCFF was uploaded into the USGS NWIS database at the end of every week of sample collection.

Prior to sample collection, polyethylene sample bottles were pre-rinsed three times with deionized water and then once with native sample water before sample collection. Samples requiring acidification were acidified to a $\mathrm{pH}$ of between 2 and 1 with the appropriate acids by using ampoules of certified, traceable concentrated acids obtained from the NWQL.

Temperature-sensitive samples were stored on ice prior to and during daily shipping to the various laboratories. The nontemperature-sensitive samples for analysis of tritium, stable isotopes of hydrogen and oxygen in water, and dissolved noble gases were shipped monthly. Temperature-sensitive or time-sensitive samples for analysis of VOCs, pesticides and pesticide degradates, perchlorate, trace elements, nutrients, major and minor ions, silica, TDS, laboratory alkalinity, radon-222, and gross alpha and gross beta radioactivity were shipped daily whenever possible. Temperature-sensitive samples to be analyzed for arsenic and iron species and non-temperature sensitive samples for analysis of chromium species were shipped weekly. The temperature-sensitive samples for stable isotopes of carbon in dissolved inorganic carbon and carbon-14 abundance were stored on ice, archived in a laboratory refrigerator, and shipped after all of the laboratory alkalinity measurements were received. Samples for analysis of uranium isotopes and isotopes of boron and strontium dissolved in water were stored at room temperature in the office until all groundwater samples were collected and results from the trace-element analyses were obtained; a subset of samples was selected for analyses, based on total uranium, boron, or strontium concentrations.

Detailed sampling protocols for individual analyses and groups of analytes are described in Koterba and others (1995), the NFM (Wilde, 2009), and in the references for analytical methods listed in table A1; brief descriptions, in the order of sample collection, are given here.

Samples for the analysis of VOCs were collected in three 40-milliliter $(\mathrm{mL})$ sample vials that were purged with three vial volumes of unfiltered groundwater before bottomfilling to eliminate atmospheric contamination. One to one (1:1) hydrochloric acid to water solution was added as a preservative to these samples. Samples for the analysis of pesticides and pesticide degradates were collected in 1-L baked amber glass bottles. These samples were filtered through a 0.7 -micrometer $(\mu \mathrm{m})$ nominal pore-size glass fiber filter during collection.
Samples for the analysis of perchlorate were collected in a $125-\mathrm{mL}$ polystyrene bottle and then filtered in two or three $20-\mathrm{mL}$ aliquots through a $0.20-\mu \mathrm{m}$ pore-size Corning ${ }^{\circledR}$ syringe-tip disk filter into a sterilized 125 -mL bottle. Samples for analysis of stable isotopes of hydrogen and oxygen in water were collected in a $60-\mathrm{mL}$ clear glass bottle filled with unfiltered groundwater, sealed with a conical cap, and secured with electrical tape to prevent leakage and evaporation. Samples for the analysis of tritium were collected by bottomfilling one 1-L polyethylene bottle and one 1-L glass bottle with unfiltered groundwater, after first overfilling the bottles with three volumes of unfiltered groundwater.

Samples for the analysis of trace elements, major and minor ions, silica, laboratory alkalinity, and TDS required filling one $250-\mathrm{mL}$ polyethylene bottle with unfiltered groundwater and one $500-\mathrm{mL}$ and one $250-\mathrm{mL}$ polyethylene bottle with filtered groundwater. Filtration was done by using a 0.45 - $\mu \mathrm{m}$ pore-size PALL ${ }^{\circledR}$ unvented capsule filter that was pre-rinsed with $2 \mathrm{~L}$ of deionized water and then rinsed with $1 \mathrm{~L}$ of groundwater prior to sampling. Each $250-\mathrm{mL}$ filtered sample then was preserved with 7.5-Normal $(\mathrm{N})$ nitric acid. Nutrient samples were collected by filtering groundwater into $125-\mathrm{mL}$ brown polyethylene bottles.

Samples for the analysis of gross alpha and gross beta particle activities and uranium isotopes were filtered into 1-L polyethylene bottles and acidified with 7.5-N nitric acid. Samples for the analysis of arsenic and iron species were filtered into two $250-\mathrm{mL}$ polyethylene bottles that were wrapped with black plastic to prevent light exposure and preserved with 6-N hydrochloric acid. Samples for the analysis of isotopes of dissolved boron and strontium in water were filtered into $250-\mathrm{mL}$ polyethylene bottles and secured with electrical tape to prevent leakage and evaporation. Samples for the analysis of stable isotopes of carbon in dissolved inorganic carbon and carbon-14 abundance were filtered and bottomfilled into $500-\mathrm{mL}$ glass bottles that first were overfilled with three bottle volumes of filtered groundwater. Carbon isotope samples had no headspace and were sealed with conical caps to avoid atmospheric contamination.

Samples for field alkalinity titrations were collected by filtering groundwater into a $250-\mathrm{mL}$ polyethylene bottle. Field alkalinity was measured in the mobile laboratory at the well site by using Gran's titration method (Gran, 1952). Titration data were entered into PCFF, and the alkalinity and concentrations of bicarbonate $\left(\mathrm{HCO}_{3}{ }^{-}\right)$and carbonate $\left(\mathrm{CO}_{3}{ }^{2-}\right)$ were automatically calculated from the titration data by using the advanced speciation method (http://or.water.usgs.gov/alk/ methods.html) with $\mathrm{pK}_{1}=6.35, \mathrm{pK}_{2}=10.33$, and $\mathrm{pK}_{\mathrm{W}}=14$ (Stumm and Morgan, 1996).

Samples for the analysis of chromium species were collected at the wellhead by using a $10-\mathrm{mL}$ syringe with an attached $0.45-\mu \mathrm{m}$ pore-size Millex-HA disk filter. After the syringe was rinsed thoroughly and filled with groundwater, the first $4 \mathrm{~mL}$ of water were forced through the filter to waste 
(to condition the filter), and the next $2 \mathrm{~mL}$ were filtered slowly into a small centrifuge vial for analysis of total chromium. Hexavalent chromium then was collected by attaching a small cation-exchange column to the syringe filter and, after conditioning the column with $2 \mathrm{~mL}$ of sample water, an additional $2 \mathrm{~mL}$ of sample water was collected in a second centrifuge vial. Both vials were preserved with 10 microliters $(\mu \mathrm{L})$ of 7.5-N nitric acid (Ball and McClesky, 2003a, b).

For the collection of groundwater samples for the analysis of radon-222, a stainless-steel and Teflon ${ }^{\circledR}$ valve assembly was attached to the sampling port at the wellhead. The valve was closed partially to create back pressure, and a 10-mL groundwater sample was taken through a Teflon ${ }^{\circledR}$ septum on the valve assembly by using a glass syringe affixed with a stainless-steel needle. The sample was then injected into a $20-\mathrm{mL}$ vial partially filled with a scintillation mixture (mineral oil and 1,2,4-trimethylbenzene) and shaken. The vial was sealed and placed in an insulated cardboard tube to protect the sample during shipping.

Samples for analysis of dissolved noble gases were collected in $3 / 8$-inch-diameter copper tubes by using reinforced nylon tubing connected to the hose bib at the wellhead. Groundwater was flushed through the tubing to dislodge bubbles before the flow was restricted with a back-pressure valve. Clamps on either side of the copper tube then were tightened, trapping a sample of groundwater for analyses of dissolved noble gases (Weiss, 1968).

Nine laboratories performed chemical analyses for this study (table A1), although most of the analyses were performed at the NWQL or by laboratories contracted by the NWQL. The NWQL maintains a rigorous QA program (Stevenson, 2013). Laboratory QC samples, including method blanks, continuing calibration verification standards, standard reference samples, reagent spikes, external certified reference materials, and external blind proficiency samples, are analyzed regularly. Method detection limits are tested continuously, and laboratory reporting levels are updated accordingly. The NWQL maintains National Environmental Laboratory Accreditation Program (NELAP) and other certifications (http://www.nelac-institute.org/accred-labs.php). The USGS Branch of Quality Systems (BQS) maintains independent oversight of QA at the NWQL and laboratories contracted by the NWQL. The BQS also runs the National Field Quality Assurance Program (NFQA) that includes annual testing of all USGS field personnel for proficiency in making field waterquality measurements (http://bqs.usgs.gov/nfqa/). Results for analyses made at the NWQL or by laboratories contracted by the NWQL are uploaded directly into the USGS NWIS database. Results of analyses made at other laboratories are compiled in a project database and uploaded from there into the USGS NWIS database. The data are available through the USGS NWIS website (http://waterdata.usgs.gov/nwis) and the SWRCB's GeoTracker GAMA database (http://geotracker. waterboards.ca.gov/gama/).

\section{Data Reporting}

The following sections give details for the laboratory reporting conventions and determination of constituents by multiple methods or laboratories.

\section{Reporting Levels}

The laboratory reporting level (LRL) and long-term method detection level (LT-MDL) are most commonly used as thresholds for reporting analytical results. The LRL is set to minimize the reporting of false negatives (not detecting a compound when it actually is present in a sample) to less than 1 percent (Childress and others, 1999). The LRL usually is set at two times the LT-MDL. The LT-MDL is derived from the standard deviation of at least 24 method detection limit (MDL) determinations made over an extended period of time. The MDL is the minimum concentration of a substance that can be measured and reported with 99 percent confidence that the concentration is greater than zero (at the MDL there is less than a 1-percent chance of a false positive) (Childress and others, 1999; U.S. Environmental Protection Agency, 2002).

For organic constituents, concentrations between the LRL and the LT-MDL are reported as having a higher degree of uncertainty (coded by the letter "E" preceding the values in the tables and text). Estimated concentrations may result from detections outside the range of calibration standards, from detections that did not meet all laboratory QC criteria, and from samples that were diluted prior to analysis. For concentrations of VOCs and pesticides less than the LT-MDL, some values are also E-coded because there is a high certainty of detection but the precision in the result is uncertain. Methods used to analyze VOCs and pesticides are considered to be information-rich because they use gas chromatography or high-performance liquid chromatography (HPLC) with mass spectrometry detection. Compounds are identified by presence of characteristic fragmentation patterns in their mass spectra in addition to being quantified by measurement of peak areas at their associated chromatographic retention times (Childress and others, 1999). Organic constituents not detected by the NWQL are reported in the NWIS database as less than the associated reporting level $(<\mathrm{LRL})$ and as nondetections $(-)$ in this report.

E-coding is not applied to results for inorganic constituents with concentrations between the LRL and LT-MDL. The LT-MDL is generally the level used by the NWQL for reporting analytical results of inorganics, with the following exceptions: the MDL is used by the NWQL for reporting bromide and nitrite and by the USGS Trace Metal Laboratory for reporting arsenic, chromium, and iron species. Nitrate plus nitrite is reported using the interim reporting level (IRL) starting October 17, 2011, following the implementation at the NWQL of a new method for determining nitrate plus nitrite in filtered water by enzymatic reduction using 
discrete analysis (Patton and Krystalla, 2011). The minimum reporting level (MRL) is used by the NWQL for reporting total dissolved solids and by the Weck Laboratory for reporting perchlorate. The MRL is the smallest measurable concentration of a constituent that may be reliably reported by using a given analytical method (Timme, 1995). Constituents not detected by the laboratories are reported in the NWIS database as less than the associated reporting level $(<$ LT-MDL, $<\mathrm{MDL},<\mathrm{IRL}$, or $<\mathrm{MRL}$ ) and as nondetections (-) in this report.

The reporting levels listed in this report were in effect during the period that analyses were made for groundwater samples from the Hard Rock study unit. The USGS NWQL updates reporting level values for each constituent at least once a year. On October 1, 2011, reporting levels changed for some organic and inorganic constituents. Both values for each constituent are reported in tables $3 \mathrm{~A}, \mathrm{~B}, \mathrm{D}, \mathrm{E}$, and F, and the higher of the two values is reported with the groundwater quality data in tables $5,6,8,9$, and 10 .

Results for some organic and inorganic constituents are presented by using SRLs derived from assessment of data from QC samples associated with groundwater samples collected as part of the GAMA-PBP (see the appendix section titled "Detections in Blanks and Application of SRLs").

Isotopes of hydrogen, oxygen, carbon, boron, and strontium are reported by using method uncertainties (MUs). The MU generally indicates the precision of a particular analytical measurement; it gives a range of values wherein the true value is expected to be found.

The reporting limits for radiochemical constituents (carbon-14, tritium, radon-222, gross alpha and gross beta radioactivity, and uranium isotopes) are based on samplespecific critical levels $\left(\mathrm{ssL}_{\mathrm{C}}\right.$ ) (McCurdy and others, 2008). The critical level is analogous to the LT-MDL used for reporting analytical results for organic and non-radioactive inorganic constituents. Here, the critical level is defined as the minimum measured activity that indicates a positive detection of the radionuclide in the sample with less than a 5 percent probability of a false positive detection. Sample-specific critical levels are used for radiochemical measurements because the critical level is sensitive to sample size and sample yield during analytical processing and is dependent on instrument background, on counting times for the sample and background, and on the characteristics of the instrument being used and the nuclide being measured. $\mathrm{An} \mathrm{ssL}_{\mathrm{C}}$ is calculated for each sample, and the measured activity in the sample is compared to the $\mathrm{ssL}_{\mathrm{C}}$ associated with that sample. Measured activities less than the $\mathrm{ssL}_{\mathrm{C}}$ are reported as nondetections.

The analytical uncertainties associated with measurement of activities also are sensitive to sample-specific parameters, including sample size, sample yield during analytical processing, and time elapsed between sample collection and various steps in the analytical procedure, as well as parameters associated with the instrumentation. Therefore, measured activities of radioactive constituents are reported with samplespecific combined standard uncertainties (CSU). The CSU is reported at the 68 percent confidence level (1-sigma). The $\mathrm{ssL}_{\mathrm{C}}$ was not reported by the laboratory for some tritium results, in which case the CSU was used as an estimated reporting level.

\section{Notation}

Stable isotopic compositions of oxygen, carbon, boron, and hydrogen are reported as relative isotope ratios in units of per mil using the standard delta notation (Coplen and others, 2002; Coplen, 2011):

$$
\delta^{i} E=\left[\frac{R_{\text {sample }}}{R_{\text {reference }}}-1\right] \times 1,000 \text { per mil }
$$
where $\quad i \quad$ is the atomic mass of the heavier isotope of the element;
$E$ is the element ( $\mathrm{H}$ for hydrogen, $\mathrm{O}$ for oxygen, $\mathrm{B}$ for boron, $\mathrm{C}$ for carbon);
$R_{\text {sample }} \quad$ is the ratio of the abundance of the heavier isotope of the element $\left({ }^{2} \mathrm{H},{ }^{18} \mathrm{O},{ }^{11} \mathrm{~B},{ }^{13} \mathrm{C}\right)$ to the lighter isotope of the element $\left({ }^{1} \mathrm{H},{ }^{16} \mathrm{O}\right.$, ${ }^{10} \mathrm{~B},{ }^{12} \mathrm{C}$ ) in the sample; and
$R_{\text {reference }} \quad$ is the ratio of the abundance of the heavier isotope of the element to the lighter isotope of the element in the reference material.

The reference material for oxygen and hydrogen is Vienna Standard Mean Ocean Water (VSMOW), which is assigned $\delta^{18} \mathrm{O}$ and $\delta^{2} \mathrm{H}$ values of 0 per mil (note that $\delta^{2} \mathrm{H}$ is sometimes written as $\delta \mathrm{D}$ because the common name of the heavier isotope of hydrogen, hydrogen-2, is deuterium) (Coplen and others, 2002; Coplen, 2011). The reference material for carbon is Vienna Pee Dee Belemnite (VPDB), which is assigned a $\delta^{13} \mathrm{C}$ value of 0 per mil (Coplen and others, 2002; Coplen, 2011). Positive values indicate enrichment of the heavier isotope, and negative values indicate depletion of the heavier isotope compared to the ratios observed in the standard reference material. The isotopic composition of boron is similarly presented as a ratio of the abundance of boron-11 to boron-10 relative to the reference material. The isotopic composition of strontium is presented as the ratio of the abundance of atoms of the heavier isotope (strontium-87) to the lighter isotope (strontium-86) of the element. Strontium and boron isotope data were not available for inclusion in this report and will be presented in a subsequent report.

\section{Constituents on Multiple Analytical Schedules}

Seven constituents targeted in this study were measured by more than one analytical method or by more than one laboratory (table A2). The preferred analytical methods 
generally were selected on the basis of better performance or sensitivity for the constituent, or (in some cases) to provide consistency with historical data from the same method.

The field water-quality indicators - alkalinity, $\mathrm{pH}$, and specific conductance-were measured in the field and at the NWQL (table 4). Field measurements generally are considered more representative of groundwater conditions than laboratory measurements because the amount of time is limited for reactions to occur in the water sample after exposure to the atmosphere (Hem, 1985). Field values of $\mathrm{pH}$ and specific conductance were measured at each Hard Rock site. Field alkalinities were measured at only 15 percent of the Hard Rock sites due to time constraints; however, the data confirm that differences between laboratory and field alkalinity values are negligible for the purpose of this study.

For total arsenic, chromium, and iron concentrations, the approved method used by the NWQL, Schedule 1948 (table 3D), is preferred over the research methods used by the USGS Trace Metal Laboratory (table 3G). The concentrations measured by the Trace Metal Laboratory are only used to calculate ratios of redox species for arsenic, chromium, and iron. For example,

$$
\frac{C r(I I I)}{C r(V I)}=\frac{C r(T)-C r(V I)}{C r(V I)},
$$

where $\operatorname{Cr}(T) \quad$ is the total chromium concentration (measured);

$\mathrm{Cr}(\mathrm{VI}) \quad$ is the concentration of hexavalent chromium (measured); and

$\mathrm{Cr}(I I I) \quad$ is the concentration of trivalent chromium (calculated).

Tritium was measured at two laboratories: LLNL and SITL. Only tritium data from the SITL were available for reporting at the time of this publication.

\section{Quality-Assurance and Quality-Control Methods and Results}

The purpose of QA/QC is to identify which data best represent environmental conditions and which data may have been affected by contamination or bias during sample collection, processing, storage, transportation, or laboratory analysis. Four types of QC measurements were evaluated in this study: (1) blank samples were collected to assess positive bias as a result of contamination during sample handling or analysis, (2) replicate samples were collected to assess variability, (3) matrix-spike tests were done to assess positive or negative bias for organic constituents, and (4) surrogate compounds were added to samples analyzed for organic constituents to assess potential bias of laboratory analytical methods.

\section{Blanks}

The primary purposes of collecting blanks are to evaluate the magnitude of potential contamination of samples with compounds of interest during sample handling or analysis and to identify and mitigate the sources of sample contamination.

Results from blanks collected for the Hard Rock study unit and for previous GAMA study units were used to establish raised SRLs for some constituents detected in blanks. Detections reported by the laboratory with concentrations less than SRLs may have significant contamination bias. These data were flagged with an appropriate remark code (described in subsequent sections).

\section{Blank Collection and Analysis}

Blanks were collected by using blank water certified by the NWQL to contain less than the reporting levels for selected constituents investigated in the study (James A. Lewis, National Water Quality Laboratory, written commun., 2011). Nitrogen-purged, organic-free water was used for blanks of organic constituents, and inorganic-free water was used for blanks of other constituents.

Source-solution blanks were collected at the beginning of the Hard Rock study and whenever a new lot of blank water was introduced to assess potential contamination of samples during transport and analysis and potential contamination of the certified blank water obtained from the NWQL. Two source-solution blanks using nitrogen-purged, organic-free blank water were collected during the Hard Rock study and analyzed for VOCs. Two source-solution blanks using inorganic-free blank water were collected for perchlorate, trace elements, major and minor ions, silica, TDS, and laboratory alkalinity. Source-solution blanks were collected in one of the mobile laboratories by pouring blank water directly into sample containers, which were then preserved, stored, shipped, and analyzed in the same manner as the groundwater samples.

Equipment blanks were collected before the start of a study unit, or when using sampling equipment (fittings, tubings, and filters) that is not frequently used, to assess the potential sources and extent of contamination of the equipment. One equipment blank was collected during the Hard Rock study in one of the mobile laboratories by pumping blank water through the groundwater sampling equipment by using a portable QC pump. Four liters of blank water were pumped through the sampling equipment before the equipment blank was collected. The equipment blank was analyzed for VOCs, pesticides and pesticide degradates, perchlorate, trace elements, nutrients, major and minor ions, silica, TDS, laboratory alkalinity, and arsenic, chromium, and iron species.

Field blanks were collected periodically during the course of the study to assess potential contamination of samples during collection, processing, transport, and analysis. 
Field blanks were collected at sampling sites by pumping blank water through the groundwater sampling equipment (fittings, tubing, and filters) by using a portable QC pump. The samples were then processed and transported using the same protocols used for the groundwater samples. Four liters of blank water were pumped through the sampling equipment before each field blank was collected. Constituents analyzed were VOCs, pesticides and pesticide degradates, perchlorate, trace elements, nutrients, major and minor ions, silica, TDS, laboratory alkalinity, and arsenic, chromium, and iron species.

Blanks were not collected for radon-222, gross alpha and gross beta radioactivity, and uranium isotopes because the laboratory determines an $\mathrm{ssL}_{\mathrm{C}}$ value for each sample. The $\mathrm{ssL}_{\mathrm{C}}$ is the minimum measured value that indicates a non-zero amount of the radionuclide in the sample, in other words, an amount of the radionuclide that is statistically significantly greater than the amount in a blank. Blanks were not collected for tritium and dissolved noble gases. Tritium and dissolved noble gases are in the atmosphere and would dissolve into any solution used in collecting a blank, making it impractical to collect a blank for these analytes. Isotopic ratios of carbon, boron, hydrogen, oxygen, and strontium are an intrinsic property of any of these elements; therefore, the concept of a blank does not apply to these ratios.

\section{Methods for Determining and Applying Study Reporting Levels (SRLs)}

In some instances in which contamination bias is observed in a set of blanks, it may be necessary to use a raised reporting level to identify the concentration above which a result is unlikely to be significantly affected by contamination bias. SRLs for VOCs collected for the 35 GAMA-PBP study units were defined by Fram and others (2012) on the basis of the assessment of results from field blanks collected for the GAMA-PBP from May 2004 through September 2010, source-solution blanks, laboratory instrument blanks, and groundwater samples. Detections of VOCs having concentrations less than the SRLs are reported as nondetections in table 5 .

SRLs for trace-element data collected for the GAMA-PBP starting in October 2009 were defined by Davis and others (2014) on the basis of statistical assessment of results from the field blanks collected for the GAMA-PBP study units from October 2009 through March 2013. The assessment used order statistics and binomial probabilities to construct an upper confidence limit (Hahn and Meeker, 1991; Olsen and others, 2010) for the maximum concentration of constituents possibly introduced while groundwater samples were collected, handled, transported, and analyzed. Detections of trace elements having concentrations less than the SRLs are marked with $\mathrm{a} \leq$ symbol preceding the reported values in table 8 to indicate that the true value may be less than or equal to the reported value (including the possibility of being a nondetection).
SRLs for nutrients collected for the Hard Rock study unit were defined by the highest concentration measured in the field blanks. Detections of nutrients having concentrations less than the SRLs are marked with a $\leq$ symbol preceding the reported value in table 9 to indicate that the true value may be less than or equal to the reported value (including the possibility of being a nondetection).

\section{Detections in Blanks and Application of SRLs}

Table A 3 presents a summary of detections in the blanks and the SRLs applied for the Hard Rock study unit. Blanks were collected at approximately 10 percent of the sampled sites in the Hard Rock study unit: 1 VOC source-solution blank, 2 inorganic source-solution blanks, 1 equipment blank, and 12 field blanks. 1,2,4-Trimethylbenzene, barium, and ammonia were the only constituents detected in the sourcesolution blanks collected during the Hard Rock study. Sodium, five trace elements (barium, cobalt, copper, lead, and zinc), and nitrite plus nitrate were detected in the equipment blank. Two VOCs (1,2,4-trimethylbenzene and tetrachloroethene), eight trace elements (barium, cobalt, copper, iron, lead, manganese, nickel, and zinc), and three nutrients (ammonia, nitrite plus nitrate, and nitrite) were detected in at least one field blank. Constituents were not detected in blanks for the following analyte groups: pesticides and pesticide degradates; perchlorate; major and minor ions (except for one detection of sodium at a concentration equal to the LT-MDL), silica, and TDS; and arsenic, chromium, and iron species.

\section{SRLs for Organic Constituents}

Fram and others (2012) identified a set of 10 VOCs that had a tendency, based on past GAMA studies, to occur in blanks as a result of contamination. The SRLs determined by Fram and others (2012) were considered in conjunction with additional QC data for the Hard Rock study unit, as shown in table A3. As discussed below, six VOCs with established SRLs (1,2,4-trimethylbenzene, 2-butanone, acetone, carbon disulfide, tetrahydrofuran, and toluene) were detected in groundwater samples from the Hard Rock study unit, and all detections less than the SRLs were reclassified as nondetections. Chloroform has an established SRL $(0.03 \mu \mathrm{g} / \mathrm{L})$; however, the SRL applies only to samples collected using monitoring-well sampling equipment, which was not used in the Hard Rock study unit.

Tetrachloroethene does not have an established SRL because the detection frequency of the VOC in field blanks for previous study units was low. Tetrachloroethene was detected in five field blanks collected for the Hard Rock study unit at concentrations ranging from 0.025 to $0.094 \mu \mathrm{g} / \mathrm{L}$ during March 31 through July 19, 2011. Tetrachloroethene was not detected in the source-solution blanks collected on March 15, 2011, and November 16, 2011, for the two lots of blank water used during the Hard Rock study. Tetrachloroethene was detected in nine groundwater samples, none of which were 
samples collected at sites where field blanks were collected. The data suggest that contamination of the field blanks was introduced not by the handling and analysis of groundwater samples but rather by the equipment used to collect field blanks. Tetrachloroethene is a solvent used in cleaning agents and manufacturing. Contamination of tetrachloroethene in field blanks collected for the Hard Rock study unit likely did not affect groundwater samples, and determination of an SRL was not necessary. No groundwater detections of tetrachloroethene were annotated or reclassified as nondetections in the Hard Rock study unit.

For GAMA-PBP studies, most concentrations of 1,2,4-trimethylbenzene in blanks and groundwater samples were found to be correlated with the presence of equipment used for collecting radon-222 samples in field vehicles. The vials used to collect radon-222 samples contain a scintillation cocktail made of mineral oil and 1,2,4-trimethylbenzene. 1,2,4-Trimethylbenzene was detected in five blanks, of which the greatest concentration $(0.35 \mu \mathrm{g} / \mathrm{L})$ was detected in a source-solution blank. The field blank collected directly following the source-solution blank had the greatest detected concentration of 1,2,4-trimethylbenzene in field blanks $(0.33 \mu \mathrm{g} / \mathrm{L} ;$ table A3) $)$. According to the NWQL certificates of analysis for the lots of organic blank water used during this study, no VOCs were detected when tested by the NWQL (James A. Lewis, National Water Quality Laboratory, written commun., October 2010 and April 2011). Detections of 1,2,4-trimethylbenzene in groundwater from the Hard Rock study unit were below the SRL determined by Fram and others (2012) of $0.56 \mu \mathrm{g} / \mathrm{L}$, with the exception of three samples (PR-27 at $0.868 \mu \mathrm{g} / \mathrm{L}$; PR-33 at $0.755 \mu \mathrm{g} / \mathrm{L}$; and PR-35 at $0.814 \mu \mathrm{g} / \mathrm{L})$. All 65 groundwater detections were likely a result of field sources of contamination based on the detection frequency and concentrations in blanks collected by the GAMA-PBP since 2004 and therefore were reclassified as nondetections in this report and the NWIS database.

Contamination of groundwater samples and field blanks by 2-butanone, acetone, and (or) tetrahydrofuran can be attributed to presence of PVC-cement at sample collection sites (some wells are plumbed with PVC piping) or with contamination of sample collection equipment with the methanol used for cleaning equipment (Fram and others, 2012). 2-Butanone was detected in three groundwater samples (SC-07, SGU-01, and PRFP-04); acetone was detected in four groundwater samples (SC-07, SGU-01, PR-15, and PRFP-04). Tetrahydrofuran was detected in four groundwater samples (SC-07, SC-09, SGU-01, and PRFP-04) in the Hard Rock study unit. Field blanks with contamination from either of these two sources were not considered representative of the ambient groundwater. Therefore, no quality-control assessment could be made for these three solvents, and no concentration threshold could be established for the SRL. Instead of establishing an SRL, detections of these three solvents in groundwater samples were reclassified as "not analyzed." This reclassification is implemented in the USGS NWIS database by setting the data quality indicator code to "reviewed and rejected." 2-Butanone, acetone, and tetrahydrofuran were detected at such high concentrations $(31.0,19.8$, and $112 \mu \mathrm{g} / \mathrm{L}$, respectively) in the PRFP-04 sample that all VOC results for this sample were considered suspect and were coded as "reviewed and rejected." The coding caused one chloroform detection, at a concentration of $0.244 \mu \mathrm{g} / \mathrm{L}$, to be omitted from this report.

Carbon disulfide was detected in four groundwater samples in the Hard Rock study unit. One of the detections was at a concentration less than the SRL of $0.03 \mu \mathrm{g} / \mathrm{L}$ determined by Fram and others (2012): PR-10 at $0.028 \mu \mathrm{g} / \mathrm{L}$. The PR-10 result was reclassified as a nondetection in this report and the NWIS database. The likely source of carbon disulfide contamination is the gloves worn by field and laboratory personnel (Worthington and others, 2007).

Toluene was detected in five groundwater samples in the Hard Rock study unit: SC-15 at $0.176 \mu \mathrm{g} / \mathrm{L}$; SG-33 at $0.083 \mu \mathrm{g} / \mathrm{L}$; PR-09 at $0.026 \mu \mathrm{g} / \mathrm{L} ;$ PR-10 at $0.045 \mu \mathrm{g} / \mathrm{L}$; and PRFP-08 at $0.264 \mu \mathrm{g} / \mathrm{L}$. All groundwater concentrations were less than the SRL of $0.69 \mu \mathrm{g} / \mathrm{L}$ and therefore were reclassified as nondetections in this report and the NWIS database. The source of toluene contamination is uncertain.

\section{SRLs for Inorganic Constituents and Nutrients}

For the inorganic constituents, SRLs were only needed for selected trace elements and nutrients. For previous GAMA study units, concentrations of trace-element SRLs were determined primarily by Olsen and others (2010) based on a statistical assessment of quality-control results collected from May 2004 through January 2008. The concentrations and detection frequencies in field blanks collected by the GAMA-PBP since January 2008 suggest that the dataset used by Olsen and others (2010) may not be representative of conditions encountered during collection of samples in recent studies, including the Hard Rock study. GAMA SRLs were updated for results collected starting October 2009 as determined by Davis and others (2014) based on a statistical assessment similar to that used by Olsen and others (2010) and that included NWQL, BQS, and GAMA QC data. Updated GAMA SRLs were used for copper $(2.1 \mu \mathrm{g} / \mathrm{L})$, iron $(6 \mu \mathrm{g} / \mathrm{L})$, lead $(0.82 \mu \mathrm{g} / \mathrm{L})$, manganese $(0.66 \mu \mathrm{g} / \mathrm{L})$, molybdenum $(0.023 \mu \mathrm{g} / \mathrm{L})$, nickel $(0.21 \mu \mathrm{g} / \mathrm{L})$, tungsten $(0.023 \mu \mathrm{g} / \mathrm{L})$, and zinc $(6.2 \mu \mathrm{g} / \mathrm{L})$ for the Hard Rock study unit (table A3 $)$. Additionally, all groundwater results for cobalt were "reviewed and rejected" in the NWIS database and therefore censored from this dataset. Seven of these nine trace elements were detected in at least one Hard Rock field blank, and most of the detections in the field blanks were at concentrations less than the updated SRLs.

Barium was detected in three blanks: one source solution blank $(0.162 \mu \mathrm{g} / \mathrm{L})$, the equipment blank $(0.121 \mu \mathrm{g} / \mathrm{L})$, and one field blank $(0.073 \mu \mathrm{g} / \mathrm{L})$; however, it was not necessary to determine an SRL for barium based on the low detection frequency in field blanks collected for the Hard Rock study unit and previous study units. 
Cobalt and manganese were detected frequently in the blanks collected during the Hard Rock study; cobalt was detected in the equipment blank $(0.03 \mu \mathrm{g} / \mathrm{L})$ and in all 12 field blanks ( 0.09 to $0.53 \mu \mathrm{g} / \mathrm{L}$ ), and manganese was detected in all 12 field blanks ( 0.29 to $0.91 \mu \mathrm{g} / \mathrm{L}$ ). Contamination bias for these two trace elements was attributed to the capsule filters used to filter samples for analysis (Davis and others, 2014). Copper was detected in the equipment blank $(2.6 \mu \mathrm{g} / \mathrm{L})$ and nine of the field blanks ( 0.62 to $3.2 \mu \mathrm{g} / \mathrm{L}$ ). Iron was detected in two field blanks ( 3.35 and $13.3 \mu \mathrm{g} / \mathrm{L}$ ), and nickel was detected in one field blank $(0.19 \mu \mathrm{g} / \mathrm{L})$, neither of which were detected in the equipment blank. Lead was detected in the equipment blank $(0.35 \mu \mathrm{g} / \mathrm{L})$ and eight of the field blanks $(0.03$ to $0.82 \mu \mathrm{g} / \mathrm{L})$. Zinc was detected in the equipment blank $(6.2 \mu \mathrm{g} / \mathrm{L})$ and eight of the field blanks (1.56 to $8.38 \mu \mathrm{g} / \mathrm{L})$. Measured concentrations in groundwater samples that were less than the SRLs were coded with a $\leq$ symbol in table 8, except for cobalt results which were omitted from this report.

Ammonia was detected in 1 of the 2 source-solution blanks at a concentration of $0.0134 \mathrm{mg} / \mathrm{L}$ and in 4 of the 12 field blanks at concentrations $0.011,0.013,0.014$, and $0.015 \mathrm{mg} / \mathrm{L}$ (table A3). Additional QC data were reviewed for ammonia, including 2 source-solution blanks and 12 field blanks collected for the GAMA Trends sampling during February 2011 through March 2012. Trends samples are collected using the same protocols and equipment as used for the Hard Rock study unit and are collected by the same personnel. Ammonia was detected in 6 of the 12 Trends field blanks at concentrations of 0.011 to $0.014 \mathrm{mg} / \mathrm{L}, 3$ of which were changed to nondetections when re-analyzed by the NWQL. According to NWQL inorganic blank water certificates, ammonia was detected at concentrations up to $0.011 \mathrm{mg} / \mathrm{L}$ in the lots of blank water used between March 2011 and March 2012. Additionally, the BQS operates a project called the Blind Blank Project (BBP) in which BQS samples are disguised as regular blank samples to evaluate potential bias in the results reported by the NWQL on a continuous basis. A review of all available BBP ammonia results (October 2010 to February 2013) shows a 4 percent detection frequency, with concentrations ranging from 0.011 to $0.058 \mathrm{mg} / \mathrm{L}$ and all detections occurring in 2011 and 2012. The data suggest that low concentrations of ammonia may be attributable to a combination of contaminated blank water, contamination during laboratory analysis, and field factors (contamination during the handling and processing of samples or equipment); however, the concentrations of the contamination are low compared to the HAL-US for ammonia $(24.7 \mathrm{mg} / \mathrm{L})$. The SRL for ammonia was determined to be equal to the highest concentration detected in field blanks for the Hard Rock study unit, $0.015 \mathrm{mg} / \mathrm{L}$. Application of the SRL resulted in coding 10 groundwater detections as less than or equal to the reported values (table 9).

Nitrate plus nitrite was detected in the equipment blank $(0.024 \mathrm{mg} / \mathrm{L})$ and one field blank $(0.031 \mathrm{mg} / \mathrm{L})$, and nitrite was detected in three field blanks at concentrations between 0.001 and $0.0014 \mathrm{mg} / \mathrm{L}$ (table A3). A review of the NWQL inorganic blank water certificates and BQS BBP data indicated no bias from blank water contamination or laboratory analysis. Field factors could not be eliminated as a source of contamination for groundwater samples; therefore, SRLs were set for the two nutrients to the highest concentrations detected in field blanks for the Hard Rock study unit: $0.031 \mathrm{mg} / \mathrm{L}$ for nitrate plus nitrite and $0.0014 \mathrm{mg} / \mathrm{L}$ for nitrite. Application of the SRLs resulted in coding six nitrite plus nitrate detections and nine nitrite detections as less than or equal to the reported values.

\section{Replicates}

Sequential replicate samples were collected to assess the precision of the water-quality data. Estimates of data precision are needed to assess whether differences between concentrations in samples are because of differences in groundwater quality or because of variability that may result from collecting, processing, and analyzing the samples.

\section{Methods of Assessment of Replicate Samples}

Two methods for measuring variability were needed to adequately assess precision over the broad range of measured concentrations of most constituents. The variability between measured concentrations in the pairs of sequential replicate samples was represented by the standard deviation (SD) for low concentrations (pairs with mean concentration less than $[<] 5$ times the reporting level) and by relative standard deviation (RSD) for high concentrations (pairs with mean concentration greater than $[>] 5$ times the reporting level) (Anderson, 1987; Mueller and Titus, 2005). The RSD is defined as the SD divided by the mean concentration for each replicate pair of samples expressed as a percentage. The reporting level (RL) used for this purpose may be an LRL, LT-MDL, SRL, MDL, or MRL for each constituent.

Replicate pairs of analyses of all constituents (except radiochemical constituents) were evaluated as follows:

- If both values were reported as detections, the SD or RSD was calculated. Acceptable precision is defined as an SD of less than one-half of the RL (for pairs with mean concentration $<5$ times the RL) or an RSD of less than 10 percent (for pairs with mean concentration $>5$ times the RL). For comparison, an RSD of 10 percent is equivalent to a relative percent difference (RPD) of 14 percent.

- If both values were reported as nondetections, the variability was considered to be acceptable by definition.

- If one value was reported as a nondetection, and the other value was reported as a detection less than the RL, then a value of zero was substituted for the nondetection and the SD was calculated. Substituting zero for the nondetection yielded the maximum 
estimate of variability for the replicate pair. For some discussions, one-half of the RL is substituted for the nondetection when calculating the SD.

- If one value for a sample pair was reported as a nondetection and the other value was reported as a $\leq$-coded value (less than or equal to the RL), or if both values were reported as $\leq$-coded values, the variability was considered acceptable. The SD could not be calculated because the $\leq$-coded results represent a range of possible values.

- If one value was reported as a nondetection and the other value was reported as a detection greater than the RL, the variability for the pair was considered unacceptable.

Replicate pairs of analyses of radiochemical constituents were evaluated by using the following equation (McCurdy and others, 2008) to calculate the normalized absolute difference (NAD):

$$
N A D=\frac{\left|R_{1}-R_{2}\right|}{\sqrt{\left(C S U_{1}^{2}+C S U_{2}^{2}\right)}},
$$

where $R_{1}$ and $R_{2} \quad$ are the results for the two samples in the replicate pair; and

$\mathrm{CSU}_{1}$ and $\mathrm{CSU}_{2}$ are the combined standard uncertainties associated with the results.

Values less than 1.65 for the NAD correspond to a significance level $(\alpha)$ of 5 percent $(\alpha=0.05)$, indicating differences that are acceptably small and not statistically significant.

If results from replicate sample pairs indicate that precision is unacceptable for a constituent and no specific reason can be identified, then this greater variability must be considered when interpreting the data. If measured concentrations are slightly greater than a water-quality benchmark, then actual concentrations could be slightly less than that benchmark. Similarly, if measured concentrations are slightly less than a water-quality benchmark, then actual concentrations could be slightly greater than a benchmark. Also, if a constituent has high variability in replicate sample pairs, then a larger difference between concentrations measured in two samples is required to conclude that the two samples have significantly different concentrations.

Until April 2013, the GAMA-PBP generally requested re-analyses of replicate pairs for results outside the acceptable limits of precision. Requests and rerun results were recorded in an Excel spreadsheet maintained by GAMA project staff. If the new result was within acceptable precision for the method as per the laboratory analysts, the original concentration was not updated. However, if the new result was accompanied with the laboratory comment, "Use new result. New result within method precision based on multiple reruns," then the concentration was updated by GAMA project staff in the NWIS database, regardless of whether precision for the updated results for the replicate pair was better or worse. Because of the longer holding times, trace elements, major and minor ions, and nutrients were generally the only constituents able to be re-analyzed by the time the data were uploaded to the NWIS database; organic constituents or inorganic constituents past the holding times could only be verified by laboratory analysts. Original and rerun values for replicate pairs that were updated per rerun results and for which the precision changed to within the acceptable limits (which only occurred for trace elements) are included in the footnotes in table A4B.

\section{Variability in Replicate Samples}

Tables A4A-C summarize the results of replicate analyses for constituents detected in groundwater samples collected in the Hard Rock study unit. Replicate analyses were made on samples from 12 sites, approximately 10 percent of all the samples collected, except for chromium species (samples from 4 sites) and dissolved noble gases (samples from 11 sites).

Of the 2,512 replicate pairs of constituents analyzed, 1,937 pairs had no detections in either sample and thus had good agreement, and 575 pairs had detections in one or both samples of the pair. Of these 575 pairs, 37 pairs were outside the limits for acceptable precision and are listed in tables A4A-C. Results for replicate analyses for constituents that were not detected in groundwater samples are not reported.

The 85 VOCs analyzed for the Hard Rock study unit represent 1,020 replicate pairs of analyses. Eleven of the replicate pairs were composed of at least 1 detection (table A4A). All pairs had results within acceptable precision.

The 63 pesticide and pesticide degradates represent 756 replicate pairs of analyses. Six of the replicate pairs were composed of at least one detection (table A4A). Two replicate pairs were each composed of a value less than the LRL and a nondetection, which had maximum SDs (by substituting zeroes for the nondetections) greater than the acceptable limits.

Twelve replicate pairs of samples were analyzed for perchlorate (table A4A). Seven of the replicate pairs were composed of at least one detection. Results for three of the seven pairs with a detection were outside the limits of acceptable precision. One replicate pair had a concentration greater than the LRL and a nondetection. The other two replicate pairs had concentrations greater than the LRL, but the RSD was greater than 10 percent or the SD was greater than one-half of the LRL. The remaining four replicate pairs had results within acceptable precision.

The 23 trace elements represent 276 replicate pairs of analyses. Of the replicate pairs, 112 were composed of either 2 values reported as nondetections, a value reported as a nondetection and a value reported as less than or equal to the SRL, or 2 values reported as less than or equal to the SRL. Of the remaining 164 replicate pairs, 8 pairs had results outside 
the limits of acceptable precision: 2 replicate pairs (iron and zinc) consisted of an environmental value less than the SRL and a replicate value greater than the SRL, 4 replicate pairs (chromium, copper, silver, and tungsten) consisted of a nondetection and a value greater than the LT-MDL, and 2 replicate pairs had aluminum results with an SD or RSD greater than the acceptable limit (table A4B). One replicate sample was diluted, raising the LT-MDL by a factor of three for the replicate sample and not for the environmental sample (HR-PRFP-08); precision for concentrations near the LT-MDL for the environmental sample could not be evaluated for this replicate pair. Of the eight replicate pairs with results for trace elements outside the limits of acceptable precision, two replicate pairs (aluminum and zinc) originally had results within the acceptable limits, yet the samples were requested for re-analysis; at least one value in each pair was updated per the rerun results, causing the pairs to have precision outside acceptable limits. Additionally, four replicate pairs of samples analyzed for trace elements (aluminum [two pairs], boron, and lithium) were reanalyzed because the results were outside the limits of acceptable precision; at least one value in each replicate pair was updated per the rerun result, and the resulting SDs or RSDs were within acceptable limits. Original and rerun results for these six replicate pairs are listed in the footnotes in table A4B.

The 5 nutrients analyzed for the Hard Rock study unit represent 60 replicate pairs of analyses. Twenty-four of the replicate pairs were each composed of either 2 values reported as nondetections, a value reported as a nondetection and a value less than or equal to the SRL, or 2 values reported as less than or equal to the SRL. Of the remaining 36 replicate pairs, 5 pairs had results outside the limits of acceptable precision: 2 replicate pairs of ammonia results consisted of an environmental value greater than the SRL and a replicate value less than the SRL; 1 replicate pair of nitrite results consisted of a value greater than the LT-MDL and a nondetection; and 1 replicate pair of nitrite results and 1 replicate pair of orthophosphate results had an RSD or SD value greater than the acceptable precision (table A4B).

The 12 major and minor ions, silica, alkalinity, and TDS represent 144 replicate pairs of analyses, of which 142 were composed of 2 detections and had results within the limits of acceptable precision. The remaining two replicate pairs had the following iodide results: one pair consisted of nondetections, and one pair consisted of an environmental value greater than the LT-MDL and a replicate value reported as a nondetection (table A4B).

Of the 55 replicate pairs of arsenic, chromium, or iron species analyses, 26 were composed of at least 1 detection. Five of these 26 replicate pairs had results outside the limits of acceptable precision: 3 replicate pairs (total iron [2 pairs] and iron [II]) had results with an RSD or SD value greater than the acceptable limits, and 2 replicate pairs were each composed of an environmental value greater than the LT-MDL and a replicate value reported as a nondetection (table A4B).
The 4 isotopic tracers represent 48 replicate analyses. All replicate pairs had results within the limits of acceptable precision.

Eleven replicate samples were each analyzed for 5 dissolved noble gases and 1 atom ratio. Five of the 66 replicate pairs had results outside the limits of acceptable precision: 1 replicate pair of results for helium-3/helium- 4 atom ratio, 2 replicate pairs of helium-4 results, and 2 replicate pairs of neon results had RSD values greater than 10 percent (table A4B).

Twelve replicate pairs of samples were each analyzed for 6 radioactive constituents, and 1 replicate pair was analyzed for uranium isotopes ( 3 different isotopes). Most of the 75 replicate pairs of constituents yielded statistically similar results $(\mathrm{p} \leq 0.05)$, and were therefore considered acceptable. Eight replicate pairs, however, had results for the following constituents outside the limits of acceptable precision: tritium, radon-222, gross alpha radioactivity ( $72-\mathrm{hr}$ count, two pairs), gross alpha radioactivity (30-day count), gross beta radioactivity (72-hr count, two pairs), and gross beta radioactivity (30-day count). The variability of the results for the radon-222 replicate pair was likely caused by the incorrect volume of sample collected for the replicate sample as reported by the NWQL. Replicate pairs of samples collected from the grid well SG-08 had results with variability outside the limits of acceptable precision for gross alpha and beta radioactivity for the 72-hour and 30-day counts. Activities for the replicate samples were lower or not detected for all four analytes (table A4C).

Detections in environmental samples were not modified on the basis of the replicate analyses.

\section{Methods for Assessing Matrix Spikes}

The addition of a known concentration of a constituent (spike) to a replicate environmental sample enables the laboratory to determine the effect of the matrix, in this case groundwater, on the analytical technique used to measure the constituent. The known compounds added in matrix spikes are the same as those analyzed in the environmental samples. This enables an analysis of matrix interferences on a compoundby-compound basis. For this study, matrix spikes were added by the laboratory performing the analysis rather than in the field. Low and high matrix-spike recoveries may be a potential concern if the concentration of a compound in a groundwater sample is close to the health-based benchmark; a low recovery could result in a falsely measured concentration less than the health-based benchmark, whereas a high recovery could result in a falsely measured concentration greater than the healthbased benchmark. In addition, a low matrix-spike recovery may indicate that the compound might not be detected in some samples if it were present at very low concentrations.

The GAMA-PBP defined the data-quality objective range for acceptable median matrix-spike recoveries as 70 to 130 percent. Only constituents with median matrix-spike 
recoveries outside of this range were flagged as having unacceptable recoveries. For some constituents, the acceptable range of 70 to 130 percent for median matrix-spike recovery was more restrictive than the acceptable control limits for laboratory-set spike recoveries. Laboratory-set spikes are aliquots of laboratory blank water to which the same spike solution used for the matrix spikes has been added. One set spike is analyzed with each set of samples. Acceptable control limits for set spikes are defined relative to the long-term variability in recovery. For example, for many NWQL analyses, acceptable set-spike recovery is within \pm 3 F-pseudosigma of the median recovery for at least 30 set spikes (Connor and others, 1998); the smaller the F-pseudosigma, the more precise the determinations (Hoaglin, 1983).

Matrix spikes were performed for VOCs and pesticides and pesticide degradates because the analytical methods for these constituents may be susceptible to matrix interferences.

\section{Matrix-Spike Recoveries}

Tables A5A-B present a summary of matrix-spike recoveries for the Hard Rock study unit. Samples for the addition of the spike mixtures for 85 VOCs were collected at 11 sites, which is approximately 10 percent of all the wells sampled. Median matrix-spike recoveries were between 70 and 130 percent for all VOCs (table A5A). One spike sample had three unusually low or high recoveries: bromodichloromethane (minimum $=0$ percent), chloroform (maximum $=1,320$ percent), and dibromochloromethane (minimum $=20$ percent). The recoveries were affected by background concentrations of the constituents at values up to 100 times the expected spike fortifications. Additionally, two spike recoveries were outside the acceptable limit for 1,2,4-trimethylbenzene $($ minimum $=0$ percent; maximum $=$ 180 percent). All groundwater detections of the compound in the Hard Rock study unit were attributed to contamination (Fram and others, 2012), and concentrations varied between replicate samples when detected at concentrations less than the SRL. Therefore, background concentrations of 1,2,4-trimethylbenzene could not be subtracted out of the spike sample to calculate meaningful spike recoveries.

Samples for the addition of the spike mixtures for 63 pesticides and pesticide degradates were collected at 10 sites. Of the 630 spike analyses, 155 (25 percent) were outside the range of acceptable recovery (table A5B). Eleven pesticides (chlorpyrifos oxon, dichlorvos, dicrotophos, dimethoate, fenamiphos sulfoxide, hexazinone, 1-naphthol, phosmet, phosmet oxon, terbufos oxon sulfone, and tribufos) had median matrix-spike recoveries less than 70 percent, 1 of which (hexazinone) was detected in groundwater samples. Low matrix-spike recoveries may indicate that a low concentration in some samples could be reported as a nondetection. One compound (tebuthiuron) had a median matrix-spike recovery greater than 130 percent and was detected in groundwater samples. High matrix-spike recoveries may indicate that a detected concentration in some samples is greater than the true concentration.

Detections in environmental samples were not modified on the basis of the matrix-spike recovery analysis.

\section{Methods for Assessing Surrogates}

Surrogate compounds are added to environmental samples in the laboratory prior to analysis to evaluate the recovery of similar constituents. Surrogate compounds were added in the laboratory to all groundwater and QC samples that were analyzed by the NWQL for VOCs and pesticides and pesticide degradates. Surrogates are used to identify general problems that may arise during laboratory sample analysis that could affect the results for all compounds in that sample. Potential problems include matrix interferences (such as high levels of dissolved organic carbon) that produce a positive bias or incomplete laboratory recovery (possibly because of improper maintenance and calibration of analytical equipment) that produces a negative bias. A 70 to 130 percent recovery of surrogates, in general, is considered acceptable; values outside this range indicate possible problems with the processing and analysis of samples (Connor and others, 1998; Sandstrom and others, 2001).

\section{Surrogate Compound Recoveries}

Table A6 presents a summary of the surrogate recoveries for the Hard Rock study unit. The table lists the surrogate compound, the analytical schedule for which each surrogate was used, the number of analyses for field blanks and environmental samples, the number of surrogate recoveries less than 70 percent, and the number of surrogate recoveries greater than 130 percent for the blank and environmental samples. Field blanks and environmental samples were considered separately to assess whether or not the matrixes present in environmental samples affect surrogate recoveries.

Most surrogate recoveries for the blank and environmental samples were within the acceptable range of 70 to 130 percent. For VOC analyses, surrogate recoveries for 93 percent of the blanks (12 field, 1 equipment, and 1 sourcesolution) and 86 percent of the environmental samples (111 samples) were within the acceptable range. For pesticides and pesticide degradates, surrogate recoveries for 96 percent of the blanks (12 field and 1 equipment) and 95 percent of the environmental samples (110 samples) were within the acceptable range (table A6).

Detections in environmental samples were not modified on the basis of the surrogate recovery analysis, except in the following case. For the SGU-01 sample, the pesticide surrogate diazinon- $d 10$ had a 0 percent recovery. Low recoveries of this surrogate may indicate that the sample was treated with chlorine prior to collection. When chlorinated, organic samples are collected in bottles containing ascorbic acid (see appendix section titled Sample Collection and 
Analysis). The unusually low recovery of diazinon- $d 10$ and the moderate concentrations of chlorination byproducts in the SGU-01 groundwater sample suggests that the sample was treated; detected VOCs in the sample included bromochloromethane $(0.080 \mu \mathrm{g} / \mathrm{L})$, bromodichloromethane $(2.9 \mu \mathrm{g} / \mathrm{L})$, carbon disulfide $(0.044 \mu \mathrm{g} / \mathrm{L})$, chloroethane $(0.088 \mu \mathrm{g} / \mathrm{L})$, chloromethane $(0.150 \mu \mathrm{g} / \mathrm{L})$, dibromochloromethane $(2.16 \mu \mathrm{g} / \mathrm{L})$, dibromomethane $(0.228 \mu \mathrm{g} / \mathrm{L})$, dichloromethane $(0.069 \mu \mathrm{g} / \mathrm{L})$, bromoform $(2.54 \mu \mathrm{g} / \mathrm{L})$, and chloroform $(5.47 \mu \mathrm{g} / \mathrm{L})$. Chlorination of SGU-01 was not known at the time of sample collection, and the samples collected for analyses of organic constituents were not treated with ascorbic acid; therefore, all results for VOCs and pesticides for this sample are considered suspect and were coded as "reviewed and rejected."

\section{Other Quality-Control Results}

Two other laboratory QC issues arose during the analysis of samples collected for the Hard Rock study unit: the effect of holding-time violations on the results of radioactive constituent data and the effect of internal laboratory QC tests indicating potential bias for results of trace-element data.

Holding time refers to the time in calendar days from sample collection to the analysis of the sample. A holding-time violation occurs when a sample is analyzed past the given holding time for a particular analysis. Analyses for some samples in the Hard Rock study unit were completed after the holding time due to a scheduling error at the laboratory or a shipping error. A delay in the analysis at the laboratory may result in different measured activities or concentrations than what may have been present in the sample.

The gross alpha and beta radioactivity reported result is the amount measured in the sample. Radioactive decay occurs between the time of sample collection and measurement, and therefore gross alpha and gross beta radioactivity can change with time. At least one result from the analysis of gross alpha and gross beta radioactivity (72-hour counts) was analyzed 4 days after sample collection for four groundwater samples (SC-04, SG-10, PR-10, and PR-28), and 8 days after sample collection for two groundwater samples (SC-22 and SC-23). Gross alpha radioactivity (72-hour) may be lower and gross beta radioactivity (72-hour) may be lower or higher than true activities in samples analyzed past the holding time. Three gross alpha radioactivity results (for SC-09, SC-22, and SG-21) were annotated by the laboratory that the raw results may indicate potential negative bias. The gross beta radioactivity (30-day) for one sample (SC-09) was analyzed early-18 days after the 72-hour count. The results for these nine samples are footnoted in table 14B.

Laboratory bias for trace elements as indicated from internal laboratory QC tests was investigated to determine whether or not environmental data were affected. The BQS operates independent, external QA projects called the Inorganic Blind Sample Project (IBSP) and Blind Blank
Project (BBP) to monitor and evaluate the quality of results for analyses of trace elements, nutrients, major and minor ions, silica, and TDS by the NWQL. The IBSP submits standard reference samples consisting of natural matrix water samples with known concentrations of inorganic constituents (Farrar and Long, 1997). The IBSP and BBP samples are submitted to the NWQL as regular environmental and blank samples, respectively. The BQS issues quarterly summaries, reporting the amount of bias (if any) observed in the results (U.S. Geological Survey, 2013c, http://bqs.usgs.gov/ibsp/).

BQS summaries from April 2011 to April 2012 include data for the period during which samples were analyzed for the Hard Rock study unit. Of the inorganic constituents analyzed for the Hard Rock study unit, 19 were reported to have bias or variability at some point during this period. The analytical biases reported by the BQS were not significant for the data collected for the Hard Rock study unit in the context of regulatory standards. Only the two constituents (aluminum and zinc) that showed ongoing (and the greatest) bias or variability during the time which samples were analyzed for the Hard Rock study unit are discussed below.

The BQS reported high false positive rates (greater than 5 percent of samples were greater than the LT-MDL) for aluminum in BBP samples analyzed at the NWQL from February through June 2011. During the time which samples were analyzed for the Hard Rock study unit (March 2011 through March 2012), 5 of the 56 blind blanks analyzed (9 percent) had aluminum concentrations ranging from 1.77 to $40.9 \mu \mathrm{g} / \mathrm{L}$, all of which are greater than the LT-MDL. The positive bias was not seen in blanks collected by the GAMA-PBP; however, replicate samples collected for the Hard Rock study unit since January 2011 may indicate potential positive bias in groundwater samples. Two of the 12 replicate pairs for the Hard Rock study unit were re-analyzed by the NWQL due to disagreement in aluminum concentrations between the environmental and replicate samples. The Hard Rock replicate pairs with initial concentrations of 2.0 and $3.3 \mu \mathrm{g} / \mathrm{L}$ and $5.4 \mu \mathrm{g} / \mathrm{L}$ and not detected were adjusted by the laboratory to nondetections upon re-analysis.

Additionally, the BQS reported variability and positive bias (measured concentrations greater than target by an average of 11 percent) for aluminum in IBSP samples analyzed at the NWQL from October 2011 through March 2012 (U.S. Geological Survey, 2012). The IBSP samples analyzed during this period had aluminum concentrations ranging from 58.4 to $144 \mu \mathrm{g} / \mathrm{L}$. The median difference between the measured and expected concentrations was $3.6 \mu \mathrm{g} / \mathrm{L}(\mathrm{SD}=17 \mu \mathrm{g} / \mathrm{L})$. The maximum concentration of aluminum for the Hard Rock study unit was $291 \mu \mathrm{g} / \mathrm{L}$ (table 8 ), and a difference of 11 percent $(32 \mu \mathrm{g} / \mathrm{L})$ does not affect assessment of whether groundwater samples in the Hard Rock study unit have aluminum concentrations greater than or less than one-half of the MCL-CA concentration or greater than or less than the MCL-CA concentration $(1,000 \mu \mathrm{g} / \mathrm{L})$. 
The BQS reported slight positive bias for zinc in IBSP samples analyzed at the NWQL during December 2010 through May 2011 and September 2011 through March 2012; the greatest positive bias occurred September through November 2011, with measured concentrations greater than the targets by an average of 15 percent. The summaries indicated that the majority of bias occurs at concentrations greater than $800 \mu \mathrm{g} / \mathrm{L}$; the instruments used by the NWQL are optimized to achieve lower detection levels. Other issues included instrument sensitivity in late 2011, which briefly affected samples to varying degrees for zinc and other trace elements including aluminum, boron, cadmium, molybdenum, chromium, cobalt, copper, nickel, silver, and strontium. Additionally, a problem with the pipettes used by the NWQL during this time affected zinc and strontium analyses. The IBSP samples analyzed September through November 2011 had zinc concentrations ranging from 37 to 1,102 $\mu \mathrm{g} / \mathrm{L}$ (U.S. Geological Survey, 2012). The median difference between the measured and expected concentrations was $44 \mu \mathrm{g} / \mathrm{L}(\mathrm{SD}=172 \mu \mathrm{g} / \mathrm{L})$; the high $\mathrm{SD}$ is evidence of variability reported by the BQS, particularly during this time period and extending through March 2012. For the Hard Rock study unit, one measured groundwater concentration of zinc $(4,530 \mu \mathrm{g} / \mathrm{L})$ was greater than one-half of the SMCL-CA (table 8). A potential positive bias of 15 percent $(680 \mu \mathrm{g} / \mathrm{L})$ does not affect assessment of whether groundwater samples in the Hard Rock study unit have zinc concentrations greater than or less than one-half of the SMCL-CA concentration or greater than or less than the SMCL-CA concentration of $5,000 \mu \mathrm{g} / \mathrm{L}$. 
Table A1. Analytical methods used by the U.S. Geological Survey (USGS) National Water Quality Laboratory (NWOL) and contract laboratories.

[Laboratory entity codes in the USGS National Water Information System (NWIS) database for laboratories other than the USGS NWQL are given in parentheses after the laboratory names. Abbreviations: UV-Vis, ultraviolet; $\delta$, delta notation, the ratio of a heavier isotope of an element $\left({ }^{i} E\right)$ to the more common lighter isotope of that element relative to a standard reference material, expressed as per mil; USEPA, U.S. Environmental Protection Agency; NRP, National Research Program]

\begin{tabular}{|c|c|c|c|}
\hline Analyte & $\begin{array}{l}\text { Analytical } \\
\text { method }\end{array}$ & Laboratory and analytical schedule & Citation(s) \\
\hline \multicolumn{4}{|c|}{ Water-quality indicators } \\
\hline Field parameters & $\begin{array}{l}\text { Calibrated field meters and test } \\
\text { kits }\end{array}$ & USGS field measurement & $\begin{array}{l}\text { U.S. Geological Survey, } \\
\text { variously dated }\end{array}$ \\
\hline \multicolumn{4}{|c|}{ Organic constituents } \\
\hline $\begin{array}{l}\text { Pesticides and pesticide } \\
\text { degradates }\end{array}$ & $\begin{array}{l}\text { Solid-phase extraction and } \\
\text { gas chromatography/mass } \\
\text { spectrometry }\end{array}$ & NWQL, Schedule 2003 & $\begin{array}{l}\text { Zaugg and others, 1995; Lindley } \\
\text { and others, 1996; Sandstrom } \\
\text { and others, 2001; Madsen and } \\
\text { others, } 2003\end{array}$ \\
\hline
\end{tabular}

Constituent of special interest

\begin{tabular}{lccc}
\hline Perchlorate & $\begin{array}{c}\text { Liquid chromatography with } \\
\text { mass spectrometry/mass } \\
\text { spectrometry (USEPA } \\
\text { Method 331.0) }\end{array}$ & $\begin{array}{l}\text { Weck Laboratories, Inc., City of } \\
\text { Industry, California, standard } \\
\text { operating procedure ORG099.R01 }\end{array}$ & $\begin{array}{c}\text { U.S. Environmental Protection } \\
\text { Agency, 2005 }\end{array}$ \\
\end{tabular}

Inorganic constituents

\begin{tabular}{|c|c|c|c|}
\hline $\begin{array}{l}\text { Major and minor ions, trace } \\
\text { elements }\end{array}$ & $\begin{array}{l}\text { Atomic absorption spectrometry, } \\
\text { colorimetry, ion-exchange } \\
\text { chromatography, inductively- } \\
\text { coupled plasma atomic- } \\
\text { emission spectrometry and } \\
\text { mass spectrometry }\end{array}$ & NWQL, Schedule 1948 & $\begin{array}{l}\text { Fishman and Friedman, 1989; } \\
\text { Faires, 1993; Fishman, 1993; } \\
\text { McLain, 1993; American Pub- } \\
\text { lic Health Association, 1998; } \\
\text { Garbarino, 1999; Garbarino } \\
\text { and others, } 2006\end{array}$ \\
\hline Nutrients & $\begin{array}{l}\text { Alkaline persulfate digestion, } \\
\text { Kjedahl digestion, colorimetry } \\
\text { by enzymatic reduction }\end{array}$ & NWQL, Schedule 2755 & $\begin{array}{l}\text { Fishman, 1993; Patton and Krys- } \\
\text { kalla, 2003, } 2011\end{array}$ \\
\hline $\begin{array}{l}\text { Arsenic, chromium, and iron } \\
\text { species }\end{array}$ & $\begin{array}{l}\text { Various techniques of ultraviolet } \\
\text { visible (UV-Vis) spectro- } \\
\text { photometry and atomic- } \\
\text { absorbance spectroscopy }\end{array}$ & $\begin{array}{l}\text { USGS Trace Metal Laboratory, } \\
\text { Boulder, Colorado (USGSTMCO) }\end{array}$ & $\begin{array}{l}\text { Stookey, 1970; To and others, } \\
\text { 1998; Ball and McCleskey, } \\
\text { 2003a, b; McCleskey and } \\
\text { others, } 2003\end{array}$ \\
\hline \multicolumn{4}{|c|}{ Isotopes } \\
\hline $\begin{array}{l}\delta^{11} \mathrm{~B} \text { of boron dissolved in } \\
\text { water }\end{array}$ & $\begin{array}{l}\text { Negative thermal-ionization } \\
\text { mass spectrometry }\end{array}$ & $\begin{array}{l}\text { USGS NRP Metals Isotope Research } \\
\text { Laboratory, Menlo Park, Califor- } \\
\text { nia (USGSMICA) }\end{array}$ & $\begin{array}{l}\text { Vengosh and others, 1989; } \\
\text { Dwyer and Vengosh, } 2008\end{array}$ \\
\hline $\begin{array}{c}{ }^{87} \mathrm{Sr} /{ }^{86} \mathrm{Sr} \text { of strontium } \\
\text { dissolved in water }\end{array}$ & $\begin{array}{l}\text { Chemical separations of thermal- } \\
\text { ionization mass spectrometry }\end{array}$ & $\begin{array}{l}\text { USGS NRP Metals Isotope Research } \\
\text { Laboratory, Menlo Park, Califor- } \\
\text { nia (USGSMICA) }\end{array}$ & Bullen and others, 1996 \\
\hline
\end{tabular}


Table A1. Analytical methods used by the U.S. Geological Survey (USGS) National Water Quality Laboratory (NWQL) and contract laboratories.-Continued

[Laboratory entity codes in the USGS National Water Information System (NWIS) database for laboratories other than the USGS NWQL are given in parentheses after the laboratory names. Abbreviations: UV-Vis, ultraviolet; $\delta$, delta notation, the ratio of a heavier isotope of an element $\left({ }^{i} E\right)$ to the more common lighter isotope of that element relative to a standard reference material, expressed as per mil; USEPA, U.S. Environmental Protection Agency; NRP, National Research Program]

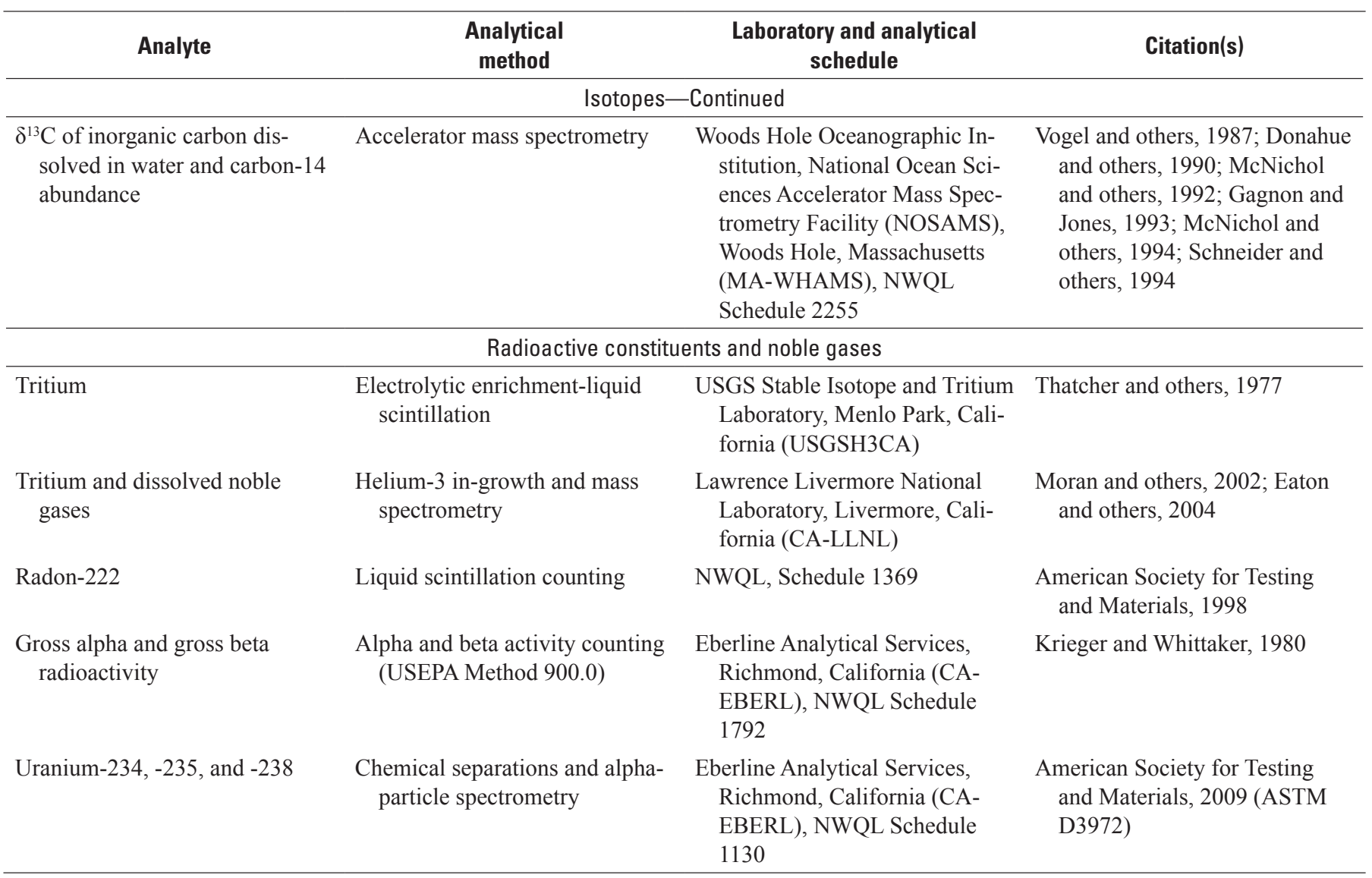

Table A2. Preferred analytical schedules for selected constituents collected for the Santa Cruz, San Gabriel, and Peninsular Ranges Hard Rock Aquifers (Hard Rock) study unit, March 2011 through March 2012, California GAMA Priority Basin Project.

[Preferred analytical schedules/methods are selected on the basis of the procedure recommended by the USGS National Water Quality Laboratory (NWQL; http://wwwnwql.cr.usgs.gov/dyn.shtml?Preferred_method_selection_procedure). Abbreviations: TML, U.S. Geological Survey Trace Metal Laboratory, Boulder, Colorado; LLNL, Lawrence Livermore National Laboratory, Livermore, California; SITL, U.S. Geological Survey Stable Isotope and Tritium Laboratory, Reston, Virginia; na, not available (no preferred schedule)]

\begin{tabular}{lccc}
\hline \multicolumn{1}{c}{ Constituent } & $\begin{array}{c}\text { Primary constituent } \\
\text { classification }\end{array}$ & Analytical schedules & Preferred analytical schedule \\
\hline Alkalinity & Results from both methods reported & field \\
Arsenic, total & Water-quality indicator & field, 1948 & 1948 \\
Chromium, total & Trace element & $1948, \mathrm{TML}$ & 1948 \\
Iron, total & Trace element & $1948, \mathrm{TML}$ & 1948 \\
pH & Trace element & $1948, \mathrm{TML}$ & field \\
Specific conductance & Water-quality indicator & field, 1948 & field \\
Tritium & Water-quality indicator & field, 1948 & na \\
\hline
\end{tabular}


Table A3. Constituents detected in field blanks and study reporting levels (SRLs) used for the Santa Cruz, San Gabriel, and Peninsular Ranges Hard Rock Aquifers (Hard Rock) study unit, March 2011 through March 2012, California GAMA Priority Basin Project.

[Sources of the SRLs: each SRL was selected from either: (1) an analysis of quality-control data from May 2004 through September 2010 for volatile organic compounds (Fram and others, 2012), or (2) an analysis of quality-control data collected October 2009 through March 2013 for trace elements (Davis and others, 2014). Abbreviations: $\leq$, less than or equal to; $\mu \mathrm{g} / \mathrm{L}$, micrograms per liter; mg/L, milligrams per liter; - , not detected; na, not available; NWIS, U.S. Geological Survey (USGS) National Water Information System]

\begin{tabular}{|c|c|c|c|c|c|}
\hline Constituent & $\begin{array}{c}\text { Number of field blank } \\
\text { detections/total } \\
\text { number of field blanks }\end{array}$ & $\begin{array}{l}\text { Concentrations } \\
\text { detected in } \\
\text { field blanks }\end{array}$ & $\begin{array}{c}\text { SRL } \\
\text { concentration }\end{array}$ & $\begin{array}{c}\text { Source } \\
\text { of } \\
\text { SRL }\end{array}$ & $\begin{array}{l}\text { Number of groundwater } \\
\text { results } \leq \text {-coded or coded as } \\
\text { "reviewed and rejected" }\end{array}$ \\
\hline \multicolumn{6}{|c|}{ Volatile organic compounds (VOCs) ( $\mu \mathrm{g} / \mathrm{L})$} \\
\hline $1,2,4$-Trimethylbenzene & $4 / 12$ & $0.027,0.032,0.055,0.33$ & ${ }^{1} 0.87$ & Fram and others, 2012 & 66 \\
\hline 2-Butanone & $0 / 12$ & - & all detections & Fram and others, 2012 & 3 \\
\hline Acetone & $0 / 12$ & - & all detections & Fram and others, 2012 & 4 \\
\hline Carbon disulfide & $0 / 12$ & - & 0.03 & Fram and others, 2012 & 1 \\
\hline Tetrachloroethene & $5 / 12$ & $0.025,0.034,0.048,0.075,0.094$ & na $^{2}$ & Fram and others, 2012 & 0 \\
\hline Tetrahydrofuran & $0 / 12$ & - & all detections & Fram and others, 2012 & 4 \\
\hline Toluene & $0 / 12$ & - & 0.69 & Fram and others, 2012 & 5 \\
\hline \multicolumn{6}{|c|}{ Trace elements $(\mu \mathrm{g} / \mathrm{L})$} \\
\hline Barium & $1 / 12$ & 0.073 & na & Davis and others, 2014 & 0 \\
\hline Cobalt & $12 / 12$ & $\begin{array}{c}0.09,0.15,0.16,0.18,0.20,0.21,0.23,0.32,0.36 \\
0.37,0.52,0.53\end{array}$ & all results & Davis and others, 2014 & 112 \\
\hline Copper & $9 / 12$ & $0.62,0.75,0.82,0.95,1.8,1.8,2.1,3.2,3.2$ & 2.1 & Davis and others, 2014 & 36 \\
\hline Iron & $2 / 12$ & $3.35,13.3$ & 6 & Davis and others, 2014 & 17 \\
\hline Lead & $8 / 12$ & $0.03,0.04,0.08,0.10,0.21,0.35,0.48,0.82$ & 0.82 & Davis and others, 2014 & 82 \\
\hline Manganese & $12 / 12$ & $\begin{array}{c}0.29,0.31,0.36,0.38,0.38,0.40,0.43,0.54,0.59 \\
0.64,0.88,0.91\end{array}$ & 0.66 & Davis and others, 2014 & 24 \\
\hline Molybdenum $^{3}$ & $0 / 12$ & - & 0.023 & Davis and others, 2014 & 0 \\
\hline Nickel & $1 / 12$ & 0.19 & 0.21 & Davis and others, 2014 & 27 \\
\hline Tungsten $^{3}$ & $0 / 12$ & - & 0.023 & Davis and others, 2014 & 18 \\
\hline Zinc & $8 / 12$ & $1.56,1.74,1.89,2.17,3.03,3.70,7.16,8.38$ & 6.2 & Davis and others, 2014 & 29 \\
\hline \multicolumn{6}{|c|}{ Nutrients (mg/L) } \\
\hline Ammonia, as nitrogen & $4 / 12$ & $0.011,0.013,0.014,0.015$ & 0.015 & Hard Rock field blanks & 10 \\
\hline Nitrite, as nitrogen & $3 / 12$ & $0.001,0.0013,0.0014$ & 0.0014 & Hard Rock field blanks & 9 \\
\hline Nitrite plus nitrate, as nitrogen & $1 / 12$ & 0.031 & 0.031 & Hard Rock field blanks & 6 \\
\hline
\end{tabular}

${ }^{1}$ Groundwater detections of 1,2,4-trimethylbenzene at concentrations less than the established SRL of $0.56 \mu \mathrm{g} / \mathrm{L}$ were censored from this dataset. Additionally, three groundwater detections at concentrations $(0.755,0.814$, and $0.868 \mu \mathrm{g} / \mathrm{L})$ greater than the SRL were likely affected by contamination bias and therefore were reclassified as nondetections in this report and the NWIS database.

${ }^{2}$ Tetrachlorothene was detected in all three Santa Cruz field blanks and two of the four San Gabriel field blanks. Most groundwater detections occurred during sampling in the Peninsular Ranges study area. Detections in field blanks were attributed to contamination of field-blank equipment early in the study, which did not affect groundwater samples.

${ }^{3}$ SRLs for molybdenum and tungsten were determined based on quality-control results other than field blanks including laboratory blanks and the USGS Branch of Quality Systems (BQS) assessment of blind blanks (Davis and others, 2014). 
Table A4A. Quality-control summary for replicate analyses of organic constituents and perchlorate detected in samples collected for the Santa Cruz, San Gabriel, and Peninsular Ranges Hard Rock Aquifers (Hard Rock) study unit, March 2011 through March 2012, California GAMA Priority Basin Project.

[Abbreviations: SD, standard deviation; RSD, percent relative standard deviation; $\leq$, less than or equal to; $<$, less than; >, greater than; RL, reporting level; nv, no value in category]

\begin{tabular}{|c|c|c|c|c|c|c|c|}
\hline \multirow[t]{2}{*}{ Constituent } & \multirow[t]{2}{*}{$\begin{array}{c}\text { Number of } \\
\text { replicate pairs with } \\
\text { nondetections/ } \\
\text { number of replicate } \\
\text { pairs }\end{array}$} & \multirow[t]{2}{*}{$\begin{array}{l}\text { Number of SDs greater } \\
\text { than } 1 / 2 \mathrm{RL} / \text { number of } \\
\text { replicate pairs with } \\
\text { mean concentration } \\
<5 \text { times the } R \mathbf{L} \\
\text { Volatile organic com }\end{array}$} & \multicolumn{3}{|c|}{$\begin{array}{l}\text { Concentrations in replicate pairs } \\
\text { with } S D s>1 / 2 R L \text {, or replicate } \\
\text { pairs with one detection and one } \\
\text { nondetection (environmental } \\
\text { sample, replicate sample) }\end{array}$} & \multirow[t]{2}{*}{$\begin{array}{l}\text { Number of RSDs greater } \\
\text { than } 10 \text { percent/number } \\
\text { of replicate pairs with } \\
\text { mean concentration } \\
>5 \text { times the } R L\end{array}$} & \multirow[t]{2}{*}{$\begin{array}{c}\text { Concentrations in } \\
\text { replicate pairs with RSDs } \\
\text { greater than } 10 \text { percent } \\
\text { (environmental sample, } \\
\text { replicate sample) }\end{array}$} \\
\hline & & & unds (VOC & $\mathrm{g} / \mathrm{L})$ & & & \\
\hline Bromochloromethane & $12 / 12$ & nv & nv & - & - & nv & nv \\
\hline Bromodichloromethane & $11 / 12$ & $0 / 1$ & nv & - & - & nv & nv \\
\hline Bromoform (Tribromomethane) & $12 / 12$ & nv & nv & - & - & nv & $\mathrm{nv}$ \\
\hline Carbon disulfide & $11 / 12$ & $0 / 1$ & nv & - & - & nv & nv \\
\hline Carbon tetrachloride (Tetrachloromethane) & $12 / 12$ & nv & nv & - & - & nv & nv \\
\hline Chlorobenzene & $12 / 12$ & nv & nv & - & - & nv & nv \\
\hline Chloroform (Trichloromethane) & $8 / 12$ & $0 / 3$ & nv & - & - & $0 / 1$ & nv \\
\hline Dibromochloromethane & $11 / 12$ & $0 / 1$ & nv & - & - & nv & nv \\
\hline 1,3-Dichlorobenzene & $12 / 12$ & nv & nv & - & - & nv & nv \\
\hline 1,4-Dichlorobenzene & $12 / 12$ & nv & nv & - & - & nv & nv \\
\hline 1,1-Dichloroethene & $11 / 12$ & $0 / 1$ & nv & - & - & nv & nv \\
\hline Methyl tert-butyl ether (MTBE) & $12 / 12$ & nv & nv & - & - & nv & nv \\
\hline Tetrachloroethene & $11 / 12$ & $0 / 1$ & nv & - & - & nv & $\mathrm{nv}$ \\
\hline 1,2,3-Trichlorobenzene & $12 / 12$ & nv & nv & - & - & nv & nv \\
\hline 1,2,4-Trichlorobenzene & $12 / 12$ & nv & nv & - & - & nv & nv \\
\hline 1,1,1-Trichloroethane & $12 / 12$ & nv & nv & - & - & nv & nv \\
\hline Trichloroethene & $11 / 12$ & $0 / 1$ & nv & - & - & nv & nv \\
\hline Trichlorofluoromethane (CFC-11) & $11 / 12$ & $0 / 1$ & nv & - & - & nv & nv \\
\hline Trichlorotrifluoroethane (CFC-113) & $12 / 12$ & nv & nv & - & - & nv & nv \\
\hline \multicolumn{8}{|c|}{ Pesticides and pesticide degradates $(\mu \mathrm{g} / \mathrm{L})$} \\
\hline Atrazine & $11 / 12$ & $1 / 1$ & $\begin{array}{c}<0.008 \\
0.008\end{array}$ & - & - & nv & $\mathrm{nv}$ \\
\hline Carbaryl & $11 / 12$ & $0 / 1$ & nv & - & - & nv & nv \\
\hline Deethylatrazine & $11 / 12$ & $1 / 1$ & $\begin{array}{l}0.006 \\
<0.006\end{array}$ & - & - & nv & $\mathrm{nv}$ \\
\hline Desulfinylfipronil & $12 / 12$ & nv & nv & - & - & nv & nv \\
\hline 3,4-Dichloroaniline & $12 / 12$ & nv & nv & - & - & nv & nv \\
\hline
\end{tabular}


Table A4A. Quality-control summary for replicate analyses of organic constituents and perchlorate detected in samples collected for the Santa Cruz, San Gabriel, and Peninsular Ranges Hard Rock Aquifers (Hard Rock) study unit, March 2011 through March 2012, California GAMA Priority Basin Project.—Continued

[Abbreviations: SD, standard deviation; RSD, percent relative standard deviation; $\leq$, less than or equal to; $<$, less than; >, greater than; RL, reporting level; nv, no value in category]

\begin{tabular}{|c|c|c|c|c|c|c|c|}
\hline \multirow[t]{2}{*}{ Constituent } & $\begin{array}{c}\text { Number of } \\
\text { replicate pairs with } \\
\text { nondetections/ } \\
\text { number of replicate } \\
\text { pairs } \\
\end{array}$ & $\begin{array}{l}\text { Number of SDs greater } \\
\text { than } 1 / 2 R L / \text { number of } \\
\text { replicate pairs with } \\
\text { mean concentration } \\
<5 \text { times the } R L\end{array}$ & \multicolumn{3}{|c|}{$\begin{array}{l}\text { Concentrations in replicate pairs } \\
\text { with } S D s>1 / 2 R L \text {, or replicate } \\
\text { pairs with one detection and one } \\
\text { nondetection (environmental } \\
\text { sample, replicate sample) }\end{array}$} & \multirow[t]{2}{*}{$\begin{array}{l}\text { Number of RSDs greater } \\
\text { than } 10 \text { percent/number } \\
\text { of replicate pairs with } \\
\text { mean concentration } \\
>5 \text { times the } R L\end{array}$} & \multirow[t]{2}{*}{$\begin{array}{c}\text { Concentrations in } \\
\text { replicate pairs with RSDs } \\
\text { greater than } 10 \text { percent } \\
\text { (environmental sample, } \\
\text { replicate sample) }\end{array}$} \\
\hline & \multicolumn{5}{|c|}{ Pesticides and pesticide degradates $(\mu \mathrm{g} / \mathrm{L})$-Continued } & & \\
\hline 2,6-Diethylaniline & $12 / 12$ & nv & nv & - & - & nv & nv \\
\hline Fipronil sulfide & $12 / 12$ & nv & nv & - & - & nv & nv \\
\hline Fipronil sulfone & $12 / 12$ & nv & $\mathrm{nv}$ & - & - & nv & nv \\
\hline Hexazinone & $12 / 12$ & nv & nv & - & - & nv & nv \\
\hline Metolachlor & $11 / 12$ & $0 / 1$ & nv & - & - & nv & nv \\
\hline Metribuzin & $12 / 12$ & $\mathrm{nv}$ & nv & - & - & nv & nv \\
\hline Prometon & $11 / 12$ & $0 / 1$ & nv & - & - & nv & nv \\
\hline Simazine & $11 / 12$ & $0 / 1$ & nv & - & - & nv & nv \\
\hline Tebuthiuron & $12 / 12$ & $\mathrm{nv}$ & $\mathrm{nv}$ & - & - & nv & nv \\
\hline \multicolumn{8}{|c|}{ Constituent of special interest ( $\mu \mathrm{g} / \mathrm{L})$} \\
\hline Perchlorate & $5 / 12$ & $3 / 4$ & $\begin{array}{l}0.11 \\
<0.10\end{array}$ & $\begin{array}{c}0.19 \\
0.10\end{array}$ & $\begin{array}{c}0.46 \\
0.11\end{array}$ & $0 / 3$ & nv \\
\hline
\end{tabular}


Table A4B. Quality-control summary for replicate analyses of inorganic constituents, isotope tracers, and dissolved noble gases detected in samples collected for the Santa Cruz, San Gabriel, and Peninsular Ranges Hard Rock Aquifers (Hard Rock) study unit, March 2011 through March 2012, California GAMA Priority Basin Project.

[Abbreviations: SD, standard deviation; RSD, percent relative standard deviation; RL, reporting level; $\mu \mathrm{g} / \mathrm{L}$, micrograms per liter; mg/L, milligrams per liter; $\leq$, less than or equal to; <, less than; >, greater than; nv, no value in category; =, equal to; NWIS, U.S. Geological Survey (USGS) National Water Information System; NWQL, USGS National Water Quality Laboratory; - , no data]

\begin{tabular}{|c|c|c|c|c|c|c|c|c|}
\hline \multirow[t]{2}{*}{ Constituent } & \multirow[t]{2}{*}{$\begin{array}{c}\text { Number of } \\
\text { replicate pairs with } \\
\text { nondetections or } \\
\leq- \text {-coded values/number } \\
\text { of replicate pairs }\end{array}$} & \multirow[t]{2}{*}{$\begin{array}{c}\text { Number of } \\
\text { SDs }>1 / 2 \text { RL/number of } \\
\text { replicate pairs with } \\
\text { mean concentration } \\
<5 \text { times the } R L \\
\text { Trace } E\end{array}$} & \multicolumn{3}{|c|}{$\begin{array}{l}\text { Concentrations in } \\
\text { replicate pairs with } \\
\text { SDs }>1 / 2 \mathrm{RL} \\
\text { (environmental sample, } \\
\text { replicate sample) }\end{array}$} & \multirow[t]{2}{*}{$\begin{array}{c}\text { Number of replicate pairs } \\
\text { with RSDs }>10 \text { percent/ } \\
\text { number of replicate pairs } \\
\text { with mean concentrations } \\
\quad>5 \text { times the } R L\end{array}$} & \multicolumn{2}{|c|}{$\begin{array}{l}\text { Concentrations in } \\
\text { replicate pairs with } \\
\text { RSDs }>10 \text { percent } \\
\text { (environmental sample, } \\
\text { replicate sample) }\end{array}$} \\
\hline & & & ments ( $\mu \mathrm{g} / \mathrm{L})$ & & & & & \\
\hline Aluminum & $7 / 12$ & ${ }^{1} 1 / 4$ & $5.8,7.0$ & - & - & $1 / 1$ & $14.0,17.0^{2}$ & - \\
\hline Antimony & $10 / 12$ & $0 / 2$ & nv & - & - & nv & nv & - \\
\hline Arsenic & $1 / 12$ & $0 / 5$ & nv & - & - & $0 / 6$ & nv & - \\
\hline Barium & $0 / 12$ & nv & nv & - & - & $0 / 12$ & nv & - \\
\hline Beryllium & $10 / 12$ & $0 / 2$ & nv & - & - & nv & nv & - \\
\hline Boron & $0 / 12$ & ${ }^{3} 0 / 2$ & nv & - & - & $0 / 10$ & nv & - \\
\hline Cadmium & $8 / 12$ & $0 / 4$ & nv & - & - & nv & nv & - \\
\hline Chromium & $8 / 12$ & $1 / 4$ & $0.11,<0.06$ & - & - & nv & nv & - \\
\hline Copper & $9 / 12$ & $1 / 3$ & $<0.8,2.4$ & - & - & nv & nv & - \\
\hline Iron & $5 / 12$ & $1 / 1$ & $\leq 5.2,6.6$ & - & - & $0 / 6$ & nv & - \\
\hline Lead & $12 / 12$ & nv & $\mathrm{nv}$ & - & - & nv & nv & - \\
\hline Lithium & $0 / 12$ & ${ }^{4} 0 / 2$ & $\mathrm{nv}$ & - & - & $0 / 10$ & $\mathrm{nv}$ & - \\
\hline Manganese & $5 / 12$ & $0 / 1$ & $\mathrm{nv}$ & - & - & $0 / 6$ & nv & - \\
\hline Molybdenum & $0 / 12$ & $0 / 1$ & nv & - & - & $0 / 11$ & nv & - \\
\hline Nickel & $1 / 12$ & $0 / 8$ & nv & - & - & $0 / 3$ & nv & - \\
\hline Selenium & $2 / 12$ & $0 / 2$ & nv & - & - & $0 / 8$ & nv & - \\
\hline Silver & $9 / 12$ & $1 / 3$ & $<0.005,0.006$ & - & - & $\mathrm{nv}$ & nv & - \\
\hline Strontium & $0 / 12$ & nv & nv & - & - & $0 / 12$ & nv & - \\
\hline Thallium & $12 / 12$ & nv & nv & - & - & nv & nv & - \\
\hline Tungsten & $2 / 12$ & $1 / 5$ & $0.03,<0.01$ & - & - & $0 / 5$ & nv & - \\
\hline Uranium & $1 / 12$ & $0 / 1$ & nv & - & - & $0 / 10$ & nv & - \\
\hline Vanadium & $2 / 12$ & $0 / 3$ & nv & - & - & $0 / 7$ & nv & - \\
\hline Zinc & $4 / 12$ & $1 / 7$ & $\leq 6.1,9.3^{5}$ & - & - & $0 / 1$ & $\mathrm{nv}$ & - \\
\hline
\end{tabular}


Table A4B. Quality-control summary for replicate analyses of inorganic constituents, isotope tracers, and dissolved noble gases detected in samples collected for the Santa Cruz, San Gabriel, and Peninsular Ranges Hard Rock Aquifers (Hard Rock) study unit, March 2011 through March 2012, California GAMA Priority Basin Project.—Continued

[Abbreviations: SD, standard deviation; RSD, percent relative standard deviation; RL, reporting level; $\mu \mathrm{g} / \mathrm{L}$, micrograms per liter; mg/L, milligrams per liter; $\leq$, less than or equal to; <, less than; >, greater than; nv, no value in category; =, equal to; NWIS, U.S. Geological Survey (USGS) National Water Information System; NWQL, USGS National Water Quality Laboratory; - , no data; cm ${ }^{3}$ STP/g, cubic centimeters of gas at standard temperature and pressure per gram of water]

\begin{tabular}{|c|c|c|c|c|c|c|c|c|}
\hline \multirow[t]{2}{*}{ Constituent } & \multirow[t]{2}{*}{$\begin{array}{c}\text { Number of } \\
\text { replicate pairs with } \\
\text { nondetections or } \\
\leq \text {-coded values/number } \\
\text { of replicate pairs }\end{array}$} & \multirow[t]{2}{*}{$\begin{array}{c}\text { Number of } \\
\text { SDs }>1 / 2 R L / \text { number of } \\
\text { replicate pairs with } \\
\text { mean concentration } \\
<5 \text { times the } R L \\
\text { Nutri }\end{array}$} & \multicolumn{3}{|c|}{$\begin{array}{l}\text { Concentrations in } \\
\text { replicate pairs with } \\
\text { SDs }>1 / 2 \text { RL } \\
\text { (environmental sample, } \\
\text { replicate sample) }\end{array}$} & \multirow[t]{2}{*}{$\begin{array}{c}\text { Number of replicate pairs } \\
\text { with RSDs }>10 \text { percent/ } \\
\text { number of replicate pairs } \\
\text { with mean concentrations } \\
\quad>5 \text { times the } R L\end{array}$} & \multicolumn{2}{|c|}{$\begin{array}{l}\text { Concentrations in } \\
\text { replicate pairs with } \\
\text { RSDs }>10 \text { percent } \\
\text { (environmental sample, } \\
\text { replicate sample) }\end{array}$} \\
\hline & & & nts (mg/L) & & & & & \\
\hline Ammonia (as nitrogen) & $7 / 12$ & $2 / 4$ & $0.016, \leq 0.013$ & $0.031, \leq 0.012$ & - & $0 / 1$ & nv & - \\
\hline Nitrate plus nitrite (as nitrogen) & $4 / 12$ & $0 / 1$ & nv & - & - & $0 / 7$ & nv & \\
\hline Nitrite (as nitrogen) & $10 / 12$ & $2 / 2$ & $0.003,<0.001$ & $0.004,0.003$ & - & $\mathrm{nv}$ & nv & - \\
\hline $\begin{array}{l}\text { Total nitrogen }(\text { ammonia }+ \text { nitrite }+ \\
\text { nitrate }+ \text { organic nitrogen })\end{array}$ & $3 / 12$ & $0 / 2$ & nv & - & - & $0 / 7$ & nv & - \\
\hline Orthophosphate (as phosphorus) & $0 / 12$ & $0 / 5$ & nv & - & - & $1 / 7$ & $0.116,0.095$ & - \\
\hline \multicolumn{9}{|c|}{ Major and minor ions, silica, and total dissolved soilds (TDS) (mg/L) } \\
\hline Bromide & $0 / 12$ & $0 / 3$ & nv & - & - & $0 / 9$ & nv & - \\
\hline Calcium & $0 / 12$ & nv & nv & - & - & $0 / 12$ & nv & - \\
\hline Chloride & $0 / 12$ & nv & nv & - & - & $0 / 12$ & nv & - \\
\hline Fluoride & $0 / 12$ & $0 / 4$ & nv & - & - & $0 / 8$ & nv & - \\
\hline Iodide & $2 / 12$ & $1 / 4$ & $0.001,<0.001$ & - & - & $0 / 6$ & nv & - \\
\hline Magnesium & $0 / 12$ & $0 / 1$ & nv & - & - & $0 / 11$ & nv & - \\
\hline Potassium & $0 / 12$ & nv & nv & - & - & $0 / 12$ & nv & - \\
\hline Sodium & $0 / 12$ & nv & nv & - & - & $0 / 12$ & nv & - \\
\hline Sulfate & $0 / 12$ & nv & nv & - & - & $0 / 12$ & nv & - \\
\hline Silica $\left(\right.$ as $\mathrm{SiO}_{2}$ ) & $0 / 12$ & nv & nv & - & - & $0 / 12$ & nv & - \\
\hline TDS & $0 / 12$ & nv & nv & - & - & $0 / 12$ & nv & - \\
\hline \multicolumn{9}{|c|}{ Arsenic, chromium, and iron species $(\mu \mathrm{g} / \mathrm{L})$} \\
\hline Arsenic (total) & $6 / 12$ & $0 / 4$ & nv & - & - & $0 / 2$ & nv & - \\
\hline Arsenic (III) & $11 / 12$ & nv & nv & - & - & $0 / 1$ & nv & - \\
\hline Chromium (total) & $4 / 4$ & nv & nv & - & - & nv & nv & - \\
\hline Chromium (VI) & $4 / 4$ & nv & nv & - & - & nv & nv & - \\
\hline Iron (total) & $2 / 12$ & $3 / 5$ & 2,4 & $4,<2$ & 7,5 & $0 / 5$ & nv & - \\
\hline Iron (II) & ${ }^{6} 2 / 11$ & $2 / 5$ & 2,4 & $4,<2$ & - & $0 / 4$ & nv & - \\
\hline
\end{tabular}


Table A4B. Quality-control summary for replicate analyses of inorganic constituents, isotope tracers, and dissolved noble gases detected in samples collected for the Santa Cruz, San Gabriel, and Peninsular Ranges Hard Rock Aquifers (Hard Rock) study unit, March 2011 through March 2012, California GAMA Priority Basin Project.—Continued

[Abbreviations: SD, standard deviation; RSD, percent relative standard deviation; RL, reporting level; $\mu \mathrm{g} / \mathrm{L}$, micrograms per liter; mg/L, milligrams per liter; $\leq$, less than or equal to; $<$, less than; $>$, greater than; nv, no value in category; =, equal to; NWIS, U.S. Geological Survey (USGS) National Water Information System; NWQL, USGS National Water Quality Laboratory; - , no data; cm ${ }^{3}$ STP/gH ${ }_{2}$, cubic centimeters of gas at standard temperature and pressure per gram of water]

\begin{tabular}{|c|c|c|c|c|c|c|c|c|}
\hline \multirow[t]{2}{*}{ Constituent } & \multirow[t]{2}{*}{$\begin{array}{c}\text { Number of } \\
\text { replicate pairs with } \\
\text { nondetections or } \\
\leq \text {-coded values/number } \\
\text { of replicate pairs }\end{array}$} & \multirow[t]{2}{*}{$\begin{array}{l}\text { Number of } \\
\text { SDs }>1 / 2 \text { RL/number of } \\
\text { replicate pairs with } \\
\text { mean concentration } \\
<5 \text { times the } R L \\
\text { Isoto }\end{array}$} & \multicolumn{2}{|c|}{$\begin{array}{l}\text { Concentrations in } \\
\text { replicate pairs with } \\
\text { SDs }>1 / 2 \mathrm{RL} \\
\text { (environmental sample, } \\
\text { replicate sample) }\end{array}$} & & \multirow[t]{2}{*}{$\begin{array}{c}\text { Number of replicate pairs } \\
\text { with RSDs }>10 \text { percent/ } \\
\text { number of replicate pairs } \\
\text { with mean concentrations } \\
\quad>5 \text { times the } R L\end{array}$} & \multicolumn{2}{|c|}{$\begin{array}{l}\text { Concentrations in } \\
\text { replicate pairs with } \\
\text { RSDs }>10 \text { percent } \\
\text { (environmental sample, } \\
\text { replicate sample) }\end{array}$} \\
\hline & & & \multicolumn{2}{|c|}{ Isotope tracers } & & & & \\
\hline$\delta^{2} \mathrm{H}$ in water (per mil) & $0 / 12$ & $\mathrm{nv}$ & nv & - & - & $0 / 12$ & $\mathrm{nv}$ & - \\
\hline$\delta^{18} \mathrm{O}$ in water (per mil) & $0 / 12$ & nv & nv & - & - & $0 / 12$ & $\mathrm{nv}$ & - \\
\hline $\begin{array}{l}\delta^{13} \mathrm{C} \text { in dissolved inorganic carbon } \\
\quad(\text { per mil) }\end{array}$ & $0 / 12$ & $\mathrm{nv}$ & $\mathrm{nv}$ & - & - & $0 / 12$ & $\mathrm{nv}$ & - \\
\hline Carbon-14 (percent modern) & $0 / 12$ & $\mathrm{nv}$ & nv & - & - & $0 / 12$ & $\mathrm{nv}$ & - \\
\hline \multicolumn{9}{|c|}{ Dissolved noble gases } \\
\hline $\operatorname{Argon}\left(10^{4} \mathrm{~cm}^{3} \mathrm{STP} / \mathrm{gH}_{2} \mathrm{O}\right)$ & $0 / 11$ & nv & nv & - & - & $0 / 11$ & nv & - \\
\hline Helium-3/Helium-4 ( $10^{6}$ atom ratio) & $0 / 11$ & nv & nv & - & - & $1 / 11$ & $0.085,0.101$ & - \\
\hline Helium-4 $\left(10^{8} \mathrm{~cm}^{3} \mathrm{STP} / \mathrm{gH}_{2} \mathrm{O}\right)$ & $0 / 11$ & nv & nv & - & - & $2 / 11$ & $9.31,7.65$ & 248,291 \\
\hline Krypton $\left(10^{8} \mathrm{~cm}^{3} \mathrm{STP} / \mathrm{gH}_{2} \mathrm{O}\right)$ & $0 / 11$ & $\mathrm{nv}$ & nv & - & - & $0 / 11$ & nv & - \\
\hline Neon $\left(10^{7} \mathrm{~cm}^{3} \mathrm{STP} / \mathrm{gH}_{2} \mathrm{O}\right)$ & $0 / 11$ & $\mathrm{nv}$ & nv & - & - & $2 / 11$ & $2.41,3.47$ & $4.02,3.04$ \\
\hline Xenon $\left(10^{8} \mathrm{~cm}^{3} \mathrm{STP} / \mathrm{gH}_{2} \mathrm{O}\right)$ & $0 / 11$ & nv & nv & - & - & $0 / 11$ & nv & - \\
\hline
\end{tabular}

${ }^{1}$ Two replicate pairs for aluminum were re-analyzed because they were outside the limits of acceptable precision: $2.0,3.3 \mu \mathrm{g} / \mathrm{L}$ (SD = 0.87 $\mu \mathrm{g} / \mathrm{L}$ ) and 5.4, $<1.7 \mu \mathrm{g} / \mathrm{L}(\mathrm{SD}=3.2 \mu \mathrm{g} / \mathrm{L}$ with $0.85 \mu \mathrm{g} / \mathrm{L}$ substituted for the nondetection). All detections for these two replicate pairs were updated by the GAMA Program in the NWIS database to nondetections per the rerun results and accompanying comment from the NWQL: "Use new result. New result within method precision based on multiple reruns."

${ }^{2}$ This aluminum replicate pair had original values of 14.0 and $13.4 \mu \mathrm{g} / \mathrm{L}$ ( $\mathrm{RSD}=3$ percent). The replicate pair was randomly requested for re-analysis, and the replicate result was updated by the GAMA Program in the NWIS database from 13.4 to $17.0 \mu \mathrm{g} / \mathrm{L}$ (new RSD = 14 percent) per the rerun result and accompanying comment from the NWQL: "Use new result. New result within method precision based on multiple reruns."

${ }^{3}$ One replicate pair for boron was re-analyzed because it was outside the limits of acceptable precision: 104, $82 \mu \mathrm{g} / \mathrm{L}$ (RSD $=16$ percent). The replicate result was updated by the GAMA Program in the NWIS database from 82 to $96 \mu \mathrm{g} / \mathrm{L}$ (new RSD = 5 percent) per the rerun result and accompanying comment from the NWQL: "Use new result. New result within method precision based on multiple reruns."

${ }^{4}$ One replicate pair for lithium was re-analyzed because it was outside the limits of acceptable precision: 10.7, $8.33 \mu \mathrm{g} / \mathrm{L}$ (RSD $=17$ percent). The replicate result was updated by the GAMA Program in the NWIS database from 8.33 to $9.74 \mu \mathrm{g} / \mathrm{L}$ (new RSD $=7$ percent) per the rerun result and accompanying comment from the NWQL: "Use new result. New result within method precision based on multiple reruns."

${ }^{5}$ This zinc replicate pair had original values of 6.7 and $9.2 \mu \mathrm{g} / \mathrm{L}(\mathrm{SD}=1.8 \mu \mathrm{g} / \mathrm{L}$ ), which was inside the limits of acceptable precision when using the study reporting level of $6.2 \mu \mathrm{g} / \mathrm{L}$ as the $\mathrm{RL}$. However, the replicate pair was requested for re-analysis, and the environmental result was updated by the GAMA Program in the NWIS database from 6.7 to $6.1 \mu \mathrm{g} / \mathrm{L}$ per the rerun result and accompanying comment from the NWQL: "Use new result. New result within method precision based on multiple reruns." Upon application of study reporting levels for trace elements, the new value was updated to $\leq 6.1 \mu \mathrm{g} / \mathrm{L}$ (new $\mathrm{SD}=$ $4.3 \mu \mathrm{g} / \mathrm{L}$ with $3.05 \mu \mathrm{g} / \mathrm{L}$ substituted for the nondetection).

${ }^{6}$ One pair was not analyzed for iron (II) because total iron was not detected, giving a total of only 11 pairs. 
Table A4C. Quality-control summary for replicate analyses of radioactive constituents detected in samples collected for the Santa Cruz, San Gabriel, and Peninsular Ranges Hard Rock Aquifers (Hard Rock) study unit, March 2011 through March 2012, California GAMA Priority Basin Project.

[For activities of radiochemical constituents, a replicate pair of analyses is defined as acceptable if the normalized absolute difference (NAD) is less than 1.65, which corresponds to a significance level of 5 percent $(\alpha=0.05)$. Abbreviations: $>$, greater than; $\mathrm{pCi} / \mathrm{L}$, picocuries per liter; - , not detected, \pm , plus or minus; nv, no value in category]

\begin{tabular}{|c|c|c|c|}
\hline \multirow[t]{2}{*}{ Constituent } & \multirow{2}{*}{$\begin{array}{c}\text { Number of replicate pairs with } \\
\begin{array}{c}\mathbf{N A D}>\mathbf{1 . 6 5} / \text { total number of } \\
\text { replicate pairs }\end{array} \\
1 / 12\end{array}$} & \multicolumn{2}{|c|}{$\begin{array}{c}\text { Activities in replicate pairs with NAD >1.65 } \\
\text { (environmental sample, replicate sample) } \\
\text { (pCi/L) }\end{array}$} \\
\hline & & $0.93 \pm 0.35,-$ & - \\
\hline Radon-222 & $1 / 12$ & ${ }^{1} 724 \pm 42,594 \pm 35$ & - \\
\hline Gross alpha radioactivity, 72 -hour count & $2 / 12$ & ${ }^{2} 5.5 \pm 1.6,-$ & $3.82 \pm 0.98,7.4 \pm 1.3$ \\
\hline Gross alpha radioactivity, 30-day count & $1 / 12$ & ${ }^{2} 2.3 \pm 1.3,-$ & - \\
\hline Gross beta radioactivity, 30-day count & $1 / 12$ & ${ }^{2} 9.86 \pm 0.72,2.12 \pm 0.35$ & - \\
\hline Uranium-234 & $0 / 1$ & nv & - \\
\hline Uranium-235 & $0 / 1$ & $\mathrm{nv}$ & - \\
\hline Uranium-238 & $0 / 1$ & nv & - \\
\hline
\end{tabular}

${ }^{1}$ Laboratory reported the replicate sample was received with an incorrect sample volume.

${ }^{2}$ Variability is outside the acceptable limits of precision for the HR-SG-08 replicate pair for gross alpha and beta radioactivity (72-hour and 30-day counts).

${ }^{3}$ One gross beta radioactivity (72-hour count) replicate pair had unacceptable variability based on application of the replicability criteria to the raw results: $-0.65,0.66 \mathrm{pCi} / \mathrm{L}(\mathrm{NAD}=2.1)$. However, both results were classified as nondetections $\left(\mathrm{ssL}_{\mathrm{C}}=0.7\right)$ by the Eberline Analytical Services laboratory and therefore were not listed in this table.

Table A5A. Quality-control summary for matrix-spike recoveries of volatile organic compounds (VOCs) in samples collected for the Santa Cruz, San Gabriel, and Peninsular Ranges Hard Rock Aquifers (Hard Rock) study unit, March 2011 through March 2012, California GAMA Priority Basin Project.

[Acceptable recovery range is between 70 and 130 percent. Abbreviation: $\mu \mathrm{g} / \mathrm{L}$, micrograms per liter]

\begin{tabular}{|c|c|c|c|c|}
\hline Constituent & $\begin{array}{l}\text { Number of spike } \\
\text { samples }\end{array}$ & $\begin{array}{l}\text { Minimum recovery } \\
\text { (percent) }\end{array}$ & $\begin{array}{l}\text { Maximum recovery } \\
\text { (percent) }\end{array}$ & $\begin{array}{c}\text { Median recovery } \\
\text { (percent) }\end{array}$ \\
\hline Acetone & 11 & 77 & 124 & 106 \\
\hline tert-Amyl methyl ether (TAME) & 11 & 75 & 105 & 91 \\
\hline Benzene & 11 & 87 & 109 & 98 \\
\hline Bromodichloromethane ${ }^{1}$ & 11 & ${ }^{2} 0$ & 119 & 80 \\
\hline Bromoform (Tribromomethane) ${ }^{1}$ & 11 & 68 & 119 & 82 \\
\hline Bromomethane (Methyl bromide) & 11 & 73 & 128 & 108 \\
\hline$n$-Butylbenzene & 11 & 57 & 104 & 85 \\
\hline Carbon tetrachloride (Tetrachloromethane) ${ }^{1}$ & 11 & 75 & 131 & 85 \\
\hline Chlorobenzene $^{1}$ & 11 & 77 & 102 & 92 \\
\hline Chloroethane ${ }^{1}$ & 11 & 88 & 122 & 98 \\
\hline Chloroform (Trichloromethane) ${ }^{1}$ & 11 & 86 & ${ }^{2} 1,320$ & 97 \\
\hline Chloromethane ${ }^{1}$ & 11 & 83 & 144 & 116 \\
\hline 3-Chloropropene & 11 & 82 & 114 & 92 \\
\hline
\end{tabular}


Table A5A. Quality-control summary for matrix-spike recoveries of volatile organic compounds (VOCs) in samples collected for the Santa Cruz, San Gabriel, and Peninsular Ranges Hard Rock Aquifers (Hard Rock) study unit, March 2011 through March 2012, California GAMA Priority Basin Project.-Continued

[Acceptable recovery range is between 70 and 130 percent. Abbreviation: $\mu \mathrm{g} / \mathrm{L}$, micrograms per liter]

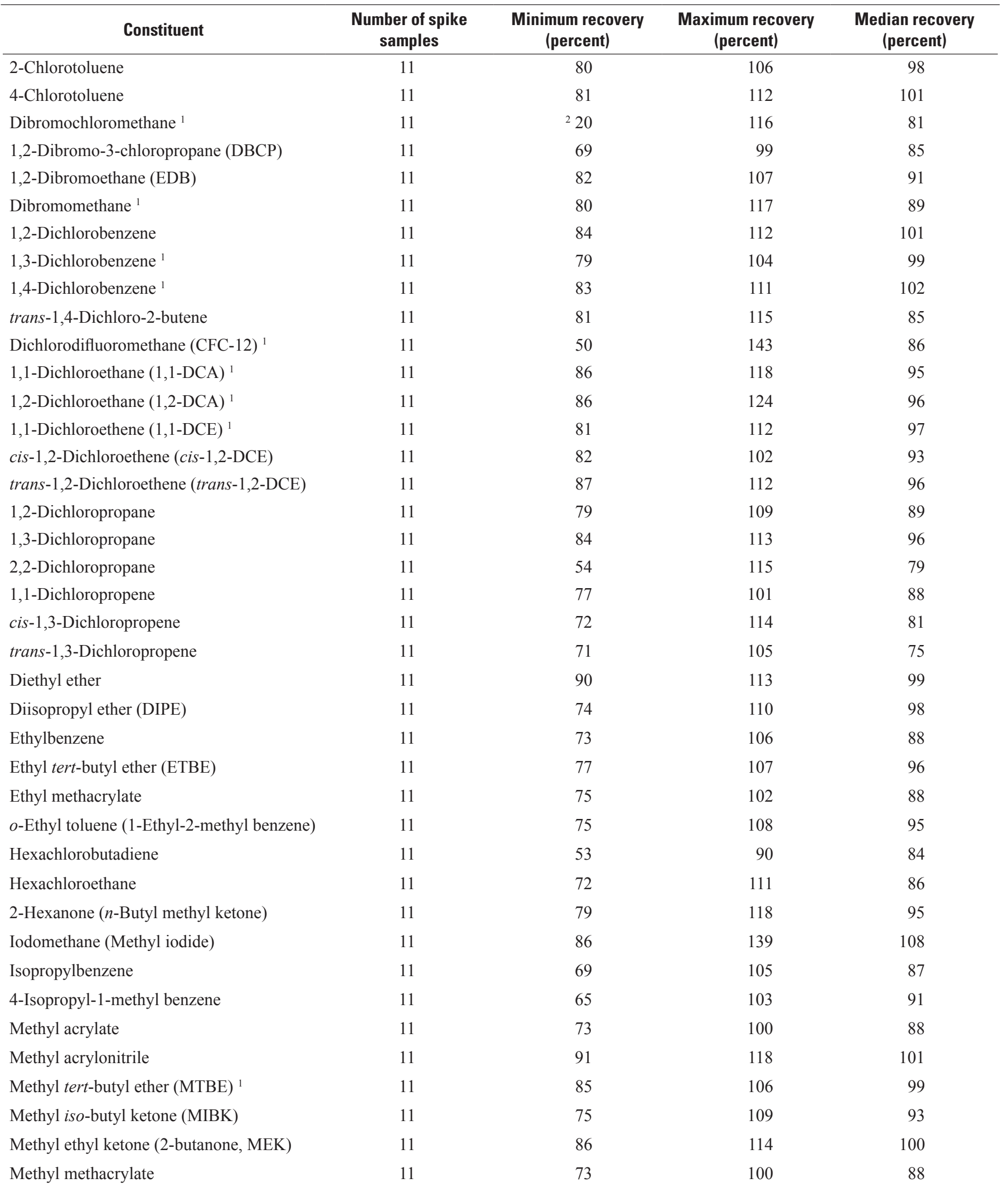


Table A5A. Quality-control summary for matrix-spike recoveries of volatile organic compounds (VOCs) in samples collected for the Santa Cruz, San Gabriel, and Peninsular Ranges Hard Rock Aquifers (Hard Rock) study unit, March 2011 through March 2012, California GAMA Priority Basin Project.-Continued

[Acceptable recovery range is between 70 and 130 percent. Abbreviation: $\mu \mathrm{g} / \mathrm{L}$, micrograms per liter]

\begin{tabular}{|c|c|c|c|c|}
\hline Constituent & $\begin{array}{l}\text { Number of spike } \\
\text { samples }\end{array}$ & $\begin{array}{l}\text { Minimum recovery } \\
\text { (percent) }\end{array}$ & $\begin{array}{c}\text { Maximum recovery } \\
\text { (percent) }\end{array}$ & $\begin{array}{l}\text { Median recovery } \\
\text { (percent) }\end{array}$ \\
\hline 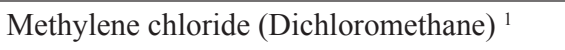 & 11 & 87 & 115 & 94 \\
\hline Naphthalene & 11 & 68 & 103 & 86 \\
\hline Perchloroethene (PCE, Tetrachloroethene) ${ }^{1}$ & 11 & 77 & 126 & 93 \\
\hline$n$-Propylbenzene & 11 & 67 & 102 & 87 \\
\hline Styrene & 11 & 54 & 104 & 87 \\
\hline 1,1,1,2-Tetrachloroethane & 11 & 74 & 104 & 83 \\
\hline 1,1,2,2-Tetrachloroethane & 11 & 77 & 112 & 88 \\
\hline Tetrahydrofuran & 11 & 86 & 114 & 101 \\
\hline 1,2,3,4-Tetramethylbenzene & 11 & 68 & 107 & 90 \\
\hline 1,2,3,5-Tetramethylbenzene & 11 & 66 & 110 & 91 \\
\hline Toluene & 11 & 84 & 110 & 101 \\
\hline 1,2,3-Trichlorobenzene ${ }^{1}$ & 11 & 77 & 106 & 96 \\
\hline 1,2,4-Trichlorobenzene ${ }^{1}$ & 11 & 74 & 99 & 91 \\
\hline $1,1,1$-Trichloroethane $(1,1,1 \text {-TCA })^{1}$ & 11 & 80 & 122 & 93 \\
\hline 1,1,2-Trichloroethane (1,1,2-TCA) & 11 & 82 & 110 & 91 \\
\hline Trichloroethene (TCE) ${ }^{1}$ & 11 & 71 & 106 & 88 \\
\hline Trichlorofluoromethane (CFC-11) ${ }^{1}$ & 11 & 92 & 135 & 105 \\
\hline 1,2,3-Trichloropropane $(1,2,3-\mathrm{TCP})$ & 11 & 82 & 106 & 95 \\
\hline Trichlorotrifluoroethane (CFC-113) ${ }^{1}$ & 11 & 81 & 126 & 92 \\
\hline 1,2,3-Trimethylbenzene & 11 & 79 & 108 & 103 \\
\hline 1,2,4-Trimethylbenzene & 11 & ${ }^{3} 0$ & ${ }^{3} 180$ & 107 \\
\hline 1,3,5-Trimethylbenzene & 11 & 73 & 106 & 92 \\
\hline Vinyl bromide (Bromoethene) & 11 & 91 & 122 & 94 \\
\hline Vinyl chloride (Chloroethene) & 11 & 83 & 146 & 89 \\
\hline$m$ - and $p$-Xylene & 11 & 75 & 107 & 96 \\
\hline$o$-Xylene & 11 & 74 & 107 & 87 \\
\hline
\end{tabular}

${ }^{1}$ Constituents detected in groundwater samples.

${ }^{2}$ Recoveries for one spike sample were affected by the high concentrations of three VOCs in the environmental sample relative to the spike concentration: bromodichloromethane, dibromochloromethane, and chloroform were detected in the environmental sample at concentrations $(6.59 \mu \mathrm{g} / \mathrm{L}, 2.00 \mu \mathrm{g} / \mathrm{L}$, and $20.5 \mu \mathrm{g} / \mathrm{L}$, respectively) up to 100 times the expected spike fortifications.

${ }^{3}$ All groundwater detections of 1,2,4-trimethylbenzene in the Hard Rock study unit were determined to be caused by contamination during the sampling process (Fram and others, 2012). Detected concentrations varied greatly within the replicate pairs and, therefore, between the environmental and spike samples. Background concentrations of the compound could not be subtracted out of the spike sample to calculate meaningful spike recoveries, resulting in two matrixspike recoveries outside the acceptable range. 
Table A5B. Quality-control summary for matrix-spike recoveries of pesticides and pesticide degradates in samples collected for the Santa Cruz, San Gabriel, and Peninsular Ranges Hard Rock Aquifers (Hard Rock) study unit, March 2011 through March 2012, California GAMA Priority Basin Project.

[Acceptable recovery range is between 70 and 130 percent]

\begin{tabular}{|c|c|c|c|c|c|}
\hline Constituent & $\begin{array}{l}\text { Number } \\
\text { of spike } \\
\text { samples }\end{array}$ & $\begin{array}{c}\text { Number of recoveries } \\
\text { outside the acceptable } \\
\text { range }\end{array}$ & $\begin{array}{l}\text { Minimum } \\
\text { recovery } \\
\text { (percent) }\end{array}$ & $\begin{array}{l}\text { Maximum } \\
\text { recovery } \\
\text { (percent) }\end{array}$ & $\begin{array}{l}\text { Median } \\
\text { recovery } \\
\text { (percent) }\end{array}$ \\
\hline Acetochlor & 10 & 0 & 76 & 120 & 105 \\
\hline Alachlor & 10 & 0 & 77 & 117 & 104 \\
\hline Atrazine ${ }^{1}$ & 10 & 0 & 86 & 127 & 107 \\
\hline Azinphos-methyl & 10 & 2 & 65 & 142 & 105 \\
\hline Benfluralin & 10 & 4 & 62 & 94 & 79 \\
\hline Carbaryl $^{1}$ & 10 & 3 & 82 & 139 & 111 \\
\hline 2-Chloro-2,6-diethylacetanilide & 10 & 0 & 82 & 117 & 100 \\
\hline 4-Chloro-2-methylphenol & 10 & 3 & 55 & 97 & 85 \\
\hline Cypermethrin & 10 & 5 & 42 & 89 & 70 \\
\hline Dacthal (DCPA) & 10 & 1 & 91 & 134 & 120 \\
\hline Deethylatrazine (2-Chloro-4-isopropylamino-6-amino-s-triazine) ${ }^{1}$ & 10 & 0 & 70 & 114 & 95 \\
\hline Desulfinylfipronil ${ }^{1}$ & 10 & 0 & 80 & 107 & 94 \\
\hline Desulfinylfipronil amide & 10 & 0 & 73 & 109 & 90 \\
\hline Diazinon & 10 & 0 & 83 & 117 & 103 \\
\hline 3,4-Dichloroaniline ${ }^{1}$ & 10 & 0 & 71 & 107 & 93 \\
\hline Dichlorvos & 10 & 10 & 14 & 56 & 24 \\
\hline Dicrotophos & 10 & 10 & 12 & 47 & 30 \\
\hline Fenamiphos & 10 & 0 & 89 & 118 & 104 \\
\hline Fenamiphos sulfone & 10 & 0 & 81 & 124 & 104 \\
\hline Fenamiphos sulfoxide & 10 & 9 & 6 & 78 & 18 \\
\hline Fipronil & 10 & 0 & 79 & 120 & 111 \\
\hline Fipronil sulfide ${ }^{1}$ & 10 & 0 & 76 & 113 & 102 \\
\hline Fipronil sulfone ${ }^{1}$ & 10 & 0 & 72 & 110 & 92 \\
\hline Fonofos & 10 & 0 & 74 & 111 & 97 \\
\hline Hexazinone $^{1}$ & 10 & 7 & 43 & 79 & 65 \\
\hline Iprodione & 10 & 4 & 58 & 97 & 78 \\
\hline Isofenphos & 10 & 1 & 69 & 123 & 99 \\
\hline Malaoxon & 10 & 2 & 58 & 104 & 85 \\
\hline Malathion & 10 & 1 & 65 & 108 & 97 \\
\hline Metalaxyl & 10 & 1 & 81 & 131 & 106 \\
\hline
\end{tabular}


Table A5B. Quality-control summary for matrix-spike recoveries of pesticides and pesticide degradates in samples collected for the Santa Cruz, San Gabriel, and Peninsular Ranges Hard Rock Aquifers (Hard Rock) study unit, March 2011 through March 2012, California GAMA Priority Basin Project.-Continued

[Acceptable recovery range is between 70 and 130 percent]

\begin{tabular}{|c|c|c|c|c|c|}
\hline Constituent & $\begin{array}{l}\text { Number } \\
\text { of spike } \\
\text { samples }\end{array}$ & $\begin{array}{c}\text { Number of recoveries } \\
\text { outside the acceptable } \\
\text { range }\end{array}$ & $\begin{array}{c}\text { Minimum } \\
\text { recovery } \\
\text { (percent) }\end{array}$ & $\begin{array}{c}\text { Maximum } \\
\text { recovery } \\
\text { (percent) }\end{array}$ & $\begin{array}{l}\text { Median } \\
\text { recovery } \\
\text { (percent }\end{array}$ \\
\hline Methidathion & 10 & 0 & 77 & 111 & 90 \\
\hline Metolachlor ${ }^{1}$ & 10 & 0 & 73 & 119 & 102 \\
\hline Metribuzin ${ }^{1}$ & 10 & 0 & 70 & 118 & 97 \\
\hline Myclobutanil & 10 & 1 & 68 & 101 & 85 \\
\hline 1-Naphthol & 10 & 8 & 4 & 96 & 29 \\
\hline Paraoxon-methyl & 10 & 4 & 46 & 102 & 72 \\
\hline Parathion-methyl & 10 & 0 & 71 & 116 & 102 \\
\hline Pendimethalin & 10 & 0 & 73 & 120 & 98 \\
\hline cis-Permethrin & 10 & 5 & 46 & 91 & 70 \\
\hline Phorate & 10 & 1 & 62 & 95 & 81 \\
\hline Phorate oxon & 10 & 1 & 67 & 116 & 95 \\
\hline Phosmet & 10 & 10 & 10 & 46 & 25 \\
\hline Phosmet oxon & 10 & 10 & 10 & 50 & 33 \\
\hline Prometon ${ }^{1}$ & 10 & 1 & 19 & 111 & 96 \\
\hline Prometryn & 10 & 0 & 73 & 121 & 97 \\
\hline Pronamide (Propyzamide) & 10 & 0 & 73 & 115 & 100 \\
\hline Simazine $^{1}$ & 10 & 0 & 80 & 123 & 100 \\
\hline Tebuthiuron $^{1}$ & 10 & 7 & 86 & 170 & 142 \\
\hline Terbufos & 10 & 1 & 62 & 101 & 86 \\
\hline Terbufos oxon sulfone & 10 & 6 & 42 & 101 & 69 \\
\hline Terbuthylazine & 10 & 0 & 86 & 128 & 107 \\
\hline Tribufos & 10 & 8 & 43 & 75 & 55 \\
\hline Trifluralin & 10 & 1 & 68 & 106 & 86 \\
\hline
\end{tabular}

${ }^{1}$ Constituent detected in groundwater samples. 
Table A6. Quality-control summary for surrogate recoveries of volatile organic compounds (VOCs) and pesticides and pesticide degradates in samples collected for the Santa Cruz, San Gabriel, and Peninsular Ranges Hard Rock Aquifers (Hard Rock) study unit, March 2011 through March 2012, California GAMA Priority Basin Project.

\begin{tabular}{|c|c|c|c|c|c|c|c|c|c|c|}
\hline \multirow[b]{2}{*}{ Surrogate } & \multirow[b]{2}{*}{$\begin{array}{l}\text { Analytical } \\
\text { schedule }\end{array}$} & \multirow[b]{2}{*}{$\begin{array}{c}\text { Constitutent } \\
\text { or constituent } \\
\text { class } \\
\text { analyzed }\end{array}$} & \multicolumn{4}{|c|}{ Surrogate information for blank samples } & \multicolumn{4}{|c|}{ Surrogate information for environmental samples } \\
\hline & & & $\begin{array}{c}\begin{array}{c}\text { Number } \\
\text { of } \\
\text { analyses }\end{array} \\
\text { 1 }\end{array}$ & $\begin{array}{l}\text { Median } \\
\text { recovery } \\
\text { (percent) }\end{array}$ & $\begin{array}{c}\text { Number of } \\
\text { surrogate } \\
\text { recoveries below } \\
70 \text { percent }\end{array}$ & $\begin{array}{c}\text { Number of } \\
\text { surrogate } \\
\text { recoveries above } \\
130 \text { percent }\end{array}$ & $\begin{array}{c}\begin{array}{c}\text { Number } \\
\text { of } \\
\text { analyses }\end{array} \\
\text { 2 }\end{array}$ & $\begin{array}{l}\text { Median } \\
\text { recovery } \\
\text { (percent) }\end{array}$ & $\begin{array}{c}\text { Number of } \\
\text { surrogate } \\
\text { recoveries below } \\
70 \text { percent }\end{array}$ & $\begin{array}{c}\text { Number of } \\
\text { surrogate } \\
\text { recoveries above } \\
130 \text { percent }\end{array}$ \\
\hline 1-Bromo-4-fluorobenzene & 2020 & VOC & 14 & 91 & 0 & 0 & 111 & 83 & 0 & 0 \\
\hline 1,2-Dichloroethane- $d 4$ & 2020 & VOC & 14 & 123 & 0 & 3 & 111 & 127 & 0 & 45 \\
\hline Toluene- $d 8$ & 2020 & VOC & 14 & 90 & 0 & 0 & 111 & 90 & 0 & 0 \\
\hline Diazinon- $d 10$ & 2003 & Pesticide & 13 & 88 & 0 & 0 & 110 & 84 & 8 & 0 \\
\hline$\alpha-\mathrm{HCH}-d 6$ & 2003 & Pesticide & 13 & 101 & 0 & 1 & 110 & 98 & 1 & 1 \\
\hline
\end{tabular}

${ }^{1}$ Total number of blank samples for VOC and pesticide analyses includes field, equipment, and source-solution blanks; a source-solution blank was not sampled for pesticide analysis.

${ }^{2}$ Three samples were omitted from this table: one environmental sample for pesticide analysis was ruined during analysis; environmental samples for VOC and pesticide analyses were chlorinated and not properly preserved, causing all results for organic constituents to be reviewed and rejected. 
Publishing support provided by the U.S. Geological Survey Science

Publishing Network, Sacramento, Tacoma, and Raleigh Publishing Service Centers

For more information concerning the research in this report, contact the Director, California Water Science Center

U.S. Geological Survey

6000 J Street, Placer Hall

Sacramento, California 95819

http://ca.water.usgs.gov 

